\title{
A QUANTITATIVE ANALYSIS OF THE SUBCELLULAR DISTRIBUTION OF HUMAN SKELETAL MUSCLE GLYCOGEN
}

\author{
A Thesis \\ Presented to \\ The Faculty of Graduate Studies \\ of \\ The University of Guelph
}

\author{
by \\ INGRID MARCHAND \\ In partial fulfilment of requirements \\ for the degree of \\ Doctor of Philosophy \\ Octobre, 2001
}

๔ Ingrid Marchand, 2001 
National Library

of Canada

Acquisitions and Bibliographic Services

305 Wellington Street OWum ON K1A ONM Canade
Bibliotheque nationale du Canada

Acquisitions et services bibliographiques

395, no Weltington Owim ON K1A ONA Canced
The author has granted a nonexclusive licence allowing the National Library of Canada to reproduce, loan, distribute or sell copies of this thesis in microform, paper or electronic formats.
L'auteur a accordé une licence non exchusive permettant à la Bibliothèque nationale du Canada de reproduire, prêter, distribuer ou vendre des copies de cette thèse sous la forme de microfiche/film, de reproduction sur papier ou sur format électronique.

The author retains ownership of the copyright in this thesis. Neither the thesis nor substantial extracts from it may be printed or otherwise reproduced without the author's permission.
L'auteur conserve la propriété du droit d'auteur qui protège cette thèse. Ni la thèse ni des extraits substantiels de celle-ci ne doivent être imprimés ou autrement reproduits sans son autorisation. 


\section{ABSTRACT}

\section{A QUANTITATIVE ANALYSIS OF THE SUBCELLULAR DISTRIBUTION OF HUMAN SKELETAL MUSCLE GLYCOGEN}

Ingrid Marchand

University of Guelph, 2001
Advisor:

Professor T.E. Graham

A few qualitative investigations suggested that subcellular distribution of human skeletal muscle glycogen is heterogeneous and that the glycogen particles in specific sites may be associated with distinct metabolic roles. This thesis investigated the subcellular distribution of human skeletal muscle glycogen. We developed a transmission electron microscopy (TEM) based technique to quantify the subcellular glycogen volume with respect to particle size and number in different subcellular compartments and fibre types. The variability for the various measures was less than $4 \%$ and the TEM-derived glycogen data had a strong correlation with that determined biochemically $\left(r^{2}=0.96, p<0.001\right)$.

Our results showed that: (1) the distribution of glycogen is heterogeneous with respect to subcellular locations and fibre types. The concentration of glycogen is greater in the subsarcolemmal than the myofibrillar compartment. Glycogen particles are smaller and more numerous in the subsarcolemmal than the myofibrillar compartment. Particles are smaller in the type I than type II fibres. The proportion of myofibrillar glycogen located in the intra-myofibrillar compartment is greater in type I than type II fibres; (2) glycogen particle sizes are normally distributed (diameter 8 to $44 \mathrm{~nm}$ ) in each subceliular location, all fibre types, and throughout a range from low to high glycogen concentrations; (3) both prolonged-moderate and short-intense exercises cause a preferential depletion of myofibrillar over subsarcolemmal glycogen; (4) short-intense 
exercise leads to the preferential net degradation of larger glycogen particles, while the net degradation of smaller ones appears minimal; (5) the glycogen resynthesis during the first $4 \mathrm{~h}$ post-exercise is principally caused by an increase in the number of glycogen particles, while the glycogen increase in the remainder of recovery (48h) is characterised by an increase in the volume of individual particles; (6) following a prolonged-moderate intensity exercise, the net rate of individual particle synthesis appears to be independent from the net rate of glycogen synthesis. Taken together, these observations are in agreement with the emerging concept of the role of subcellular compartmentalisation in the control of glycogen metabolism, and add another level of complexity to glycogen metabolism, which currently can only be addressed by use of ultrastructural analysis. 


\section{ACKNOWLEDGEMENTS}

I would like to thank my advisor Dr Terry Graham for his friendship and guidance throughout the work of this thesis. He has been a great mentor and his patience throughout the years has been exceptional. His enthusiasm for research is inspiring.

I am grateful to my thesis committee, Drs Lawrence Spriet, Jim Kirkland and Mark Tarnopolsky, for their time and expertise. Furthermore, I gratefully thank Dr Jake Barclay, for his wisdom and continual support.

I express appreciation to Dr Jacqueline Bourgeois (McMaster University), Dr Kathy Chorneyko (McMaster University) and Dr George Harauz (Guelph University) for their expertise in electron microscopy and critical input into the development of the methodology. I would particularly like to thank Dr Mark Tarnopolsky for his encouragement and generosity. The technologists from the electron microscopy department of McMaster University deserve special thanks for their time and patience during the method development.

I would like to thank NSERC and the Gatorade Sports and Science institute for the financial support that they provided throughout the years.

I could not emphasise enough the importance of my friends in making my life enjoyable and balanced: merci à tous. Special thanks to Jim and Todd for the time you spent discussing, sharing, and helping me throughout those years.

Finalement, j'aimerais remercier ma famille, ma mère, mon père, mon frère, ainsi que Evelyne et Gaby pour leurs support et encouragements. Maman et papa, votre amitiè, confiance et amour inconditionel sont la clé de ma réussite. 


\section{TABLE OF CONTENT}

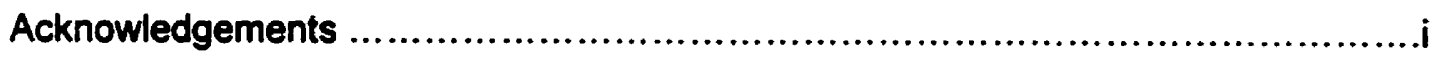

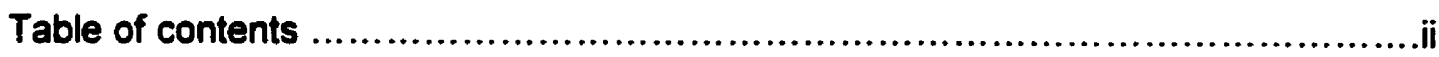

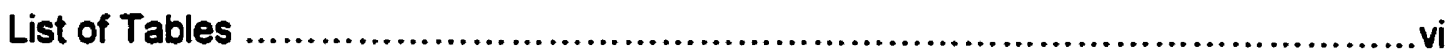

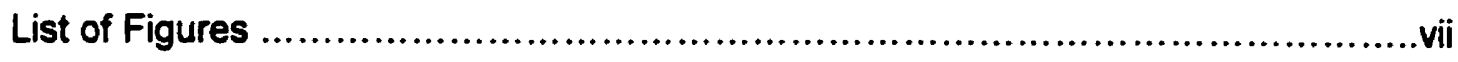

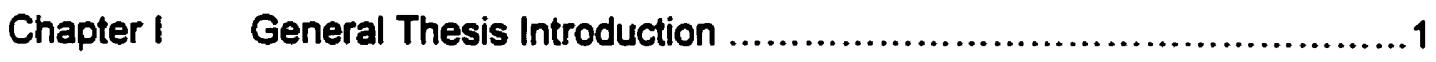

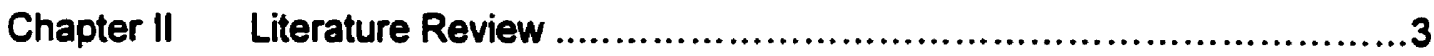

Skeletal muscle glycogen structure and function .................4

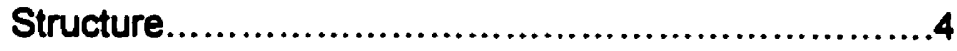

Function .................................................

Glucose disposal and glycogen metabolism in skeletal

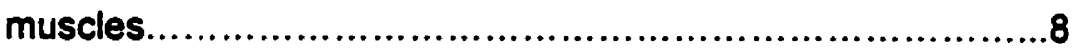

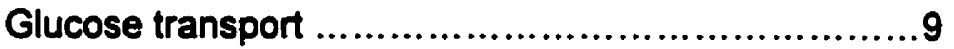

Glucose phosphorylation .................................11

Glycogen synthesis ....................................12

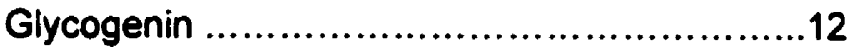

Glycogen synthase ...............................14

Branching enzyme ...................................18

Model for glycogen synthesis ......................18

Rate determining reaction in glycogen

synthesis and glucose disposal ...................20

Glycogenolysis ...............................................21

Glycogen phosphorylase and

debranching enzyme ...............................21

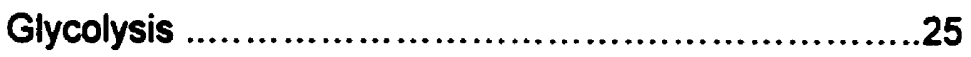

Glycogen turnover .......................................26

Fibre type differences in glycogen metabolism .................. 27

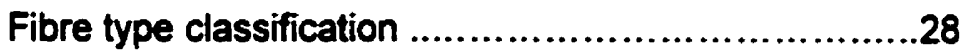

Glycogen concentration....................................29

Glycogen degradation rates...............................30

Glycogen synthesis rates .................................31

Glycosomes - the organelles of glycogen metabolism ..........32

Ultrastructural and morphological studies of glycosomes .......38 
Techniques used to enhance glycogen during transmission electron microscopic analysis ...............38

Morphology of glycogen .................................47

Size of glycogen particles .................................50

Subcellular location of glycogen in skeletal muscles ....53

Effect of exercise on glycogen distribution ...............58

Conclusion .60

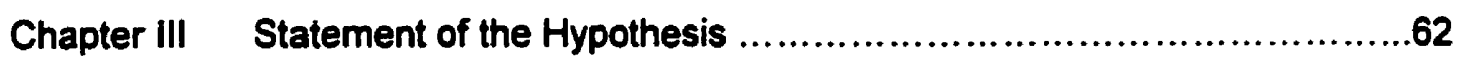

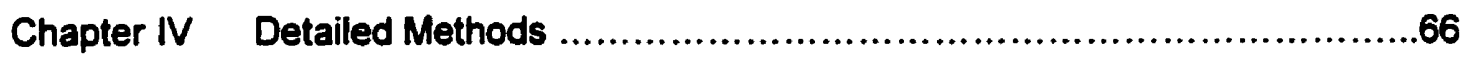

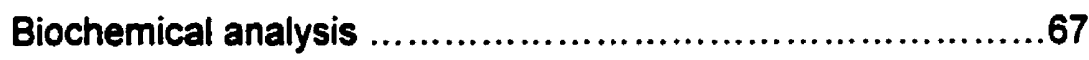

Transmission electron microscopic (TEM) analysis .............68

Digitising ....................................................79

Analysis of subcellular glycogen content

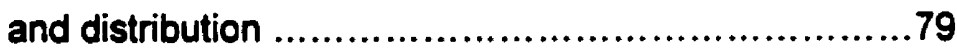

Single particle glycosome analysis ...............79

Total glycogen analysis ...........................81

Intra- and inter-myofibrillar glycogen

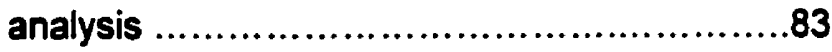

Detailed computer analysis procedures ..................85

Preparing the image ..............................85

Cleaning the image ..............................87

Calibration ..........................................92

Single particle analysis .........................93

Glycogen content analysis .......................95

Background analysis ............................97

Z-line width measurements ......................98

Image dimension measurements .................100

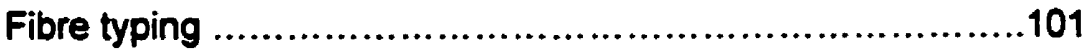

Reliability and validity testing ....................................106

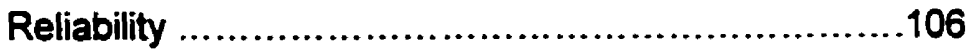

Validity .......................................................111

Calculations ......................................................114

Biochemical analysis ...................................114 
TEM analysis

Experimental design

Statistics

Chapter V Experiment 1: Quantification of subcellular glycogen in resting

human skeletal muscie: granule size, number and location .124

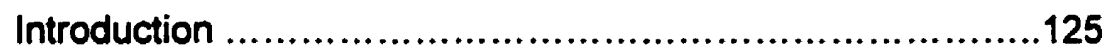

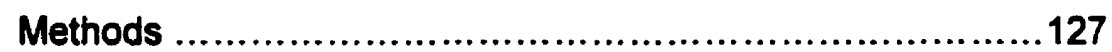

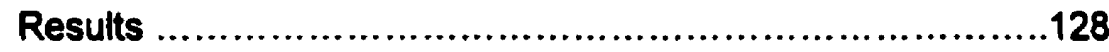

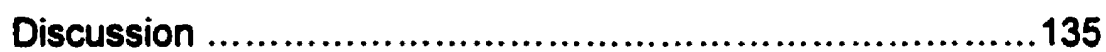

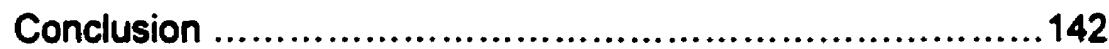

Chapter VI Experiment 2: Quantitative analysis of the intracellular distribution of glycogen in human skeletal muscle during recovery from

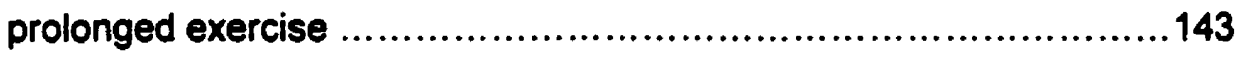

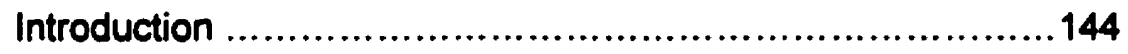

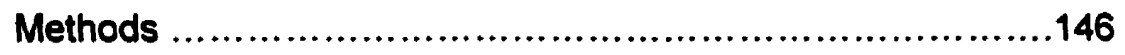

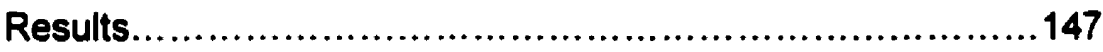

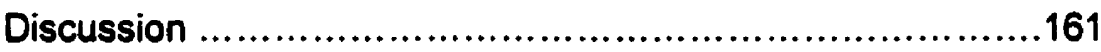

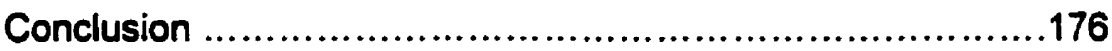

Chapter VII Experiment 3: Quantitative analysis of the changes in glycogen particle size and number in different subcellular locations in human skeletal muscle during high-intensity exercise...................177

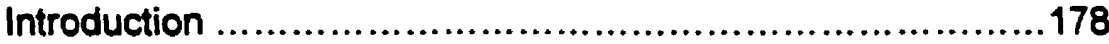

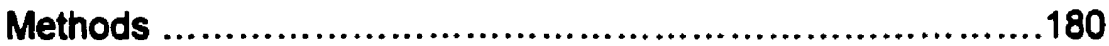

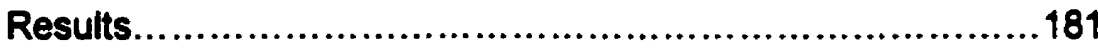

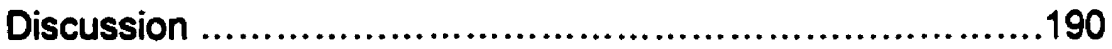

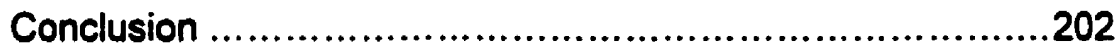

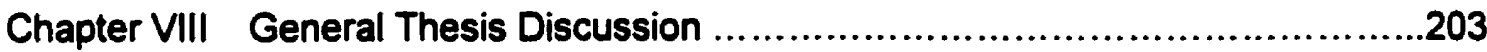

Method validity and reliability ..................................206

Frequency distribution of particle diameters ......................210

Subcellular glycogen distribution ...................................218

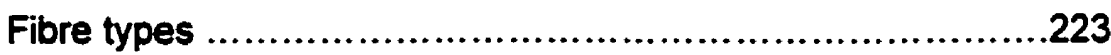

Glycogen metabolism during exercise .............................226

Limitations and future considerations .............................240

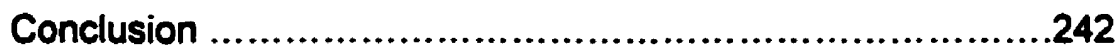


References

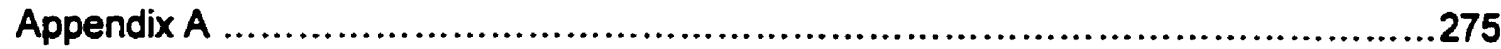

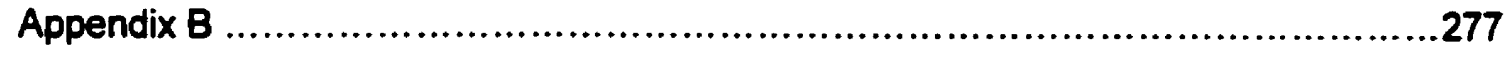

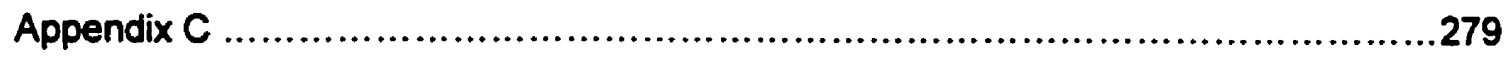

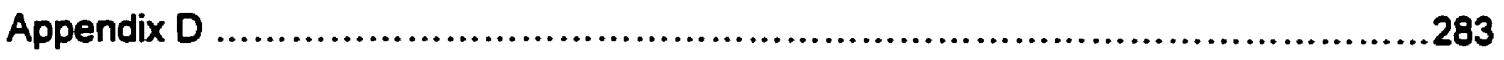

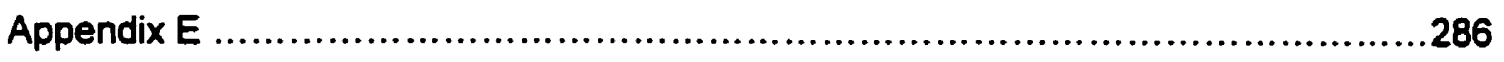

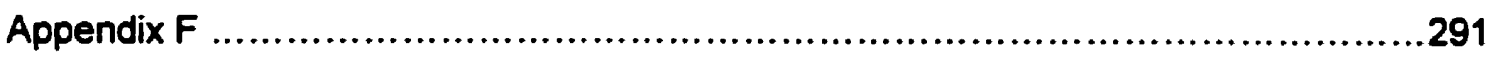




\section{LIST OF TABLES}

Table 1. Ultrastructural studies of glycogen ......................................40

Table 2. Subcellular location of the glycogen particles in human skeletal muscles ......................................................55

Table 3. Intra- and inter-observer reliability testing ..............................108

Table 4. Dependent variables .....................................................129

Table 5. Summary of the dependent variables for main effects of time, fibre type and their interaction ...........................................150

Table 6. Summary of the pre-and post-exercise values for all dependent variables .............................................................. 182

Table 7. Summary of the effect of subcellular location for all dependent variables ................................................................184

Table 8. Estimates of size and \# of glucosyl units per tier .....................211 


\section{LIST OF FIGURES}

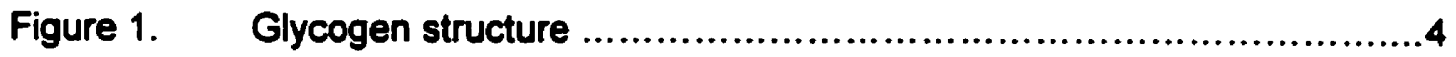

Figure 2. Pathway of glycogen biogenesis .......................................19

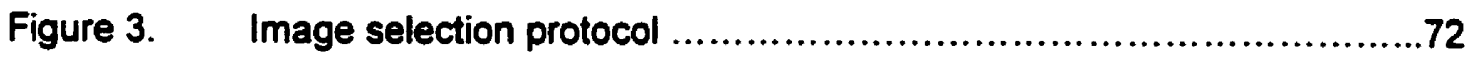

Figure 4. Illustration of the first subsarcolemmal location ...........................73

Figure 5. Illustration of the second subsarcolemmal location .......................74

Figure 6. Illustration of the third subsarcolemmal location .........................75

Figure 7. Illustration of the fourth subsarcolemmal location ........................76

Figure 8. Illustration of the fifth subsarcolemmal location ...........................77

Figure 9. Illustration of the difference between inter- and intra-myofibrillar

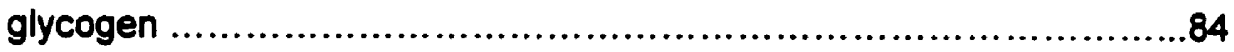

Figure 10. Illustration of a myofibrillar image immediately following digitisation ....86

Figure 11. Illustration of the same image as Figure 10, but following its

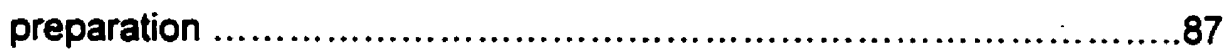

Figure 12. Illustration of the same image as Figure 11, but following the

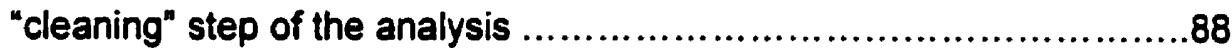

Figure 13. Illustration of a subsarcolemmal image following its preparation .........90

Figure 14. Illustration of the same image as Figure 13, but following the "cleaning" step of the analysis ...............................................91

Figure 15. Illustration of the technique used to measure the Z-line width ...........99

Figure 16. Illustration of a typical type I fibre ........................................103

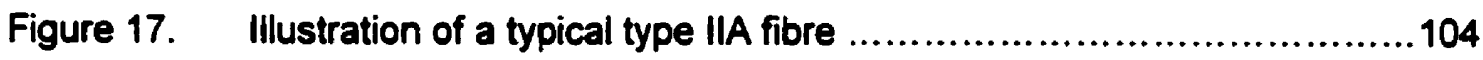

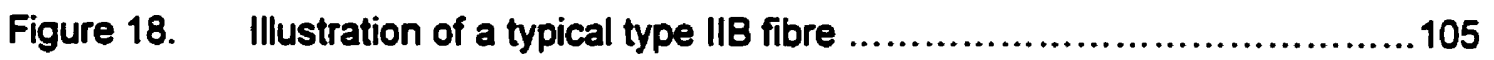

Figure 19. Comparison of the diameter of the intra- and inter-myofibrillar

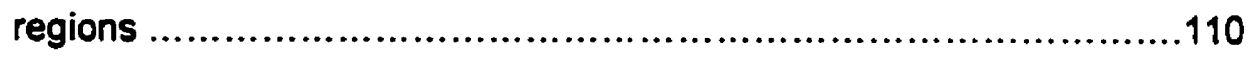

Figure 20. Correlation between biochemical and TEM-based glycogen

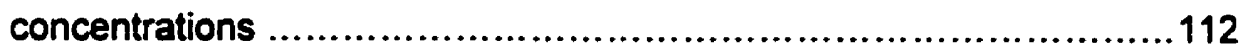

Figure 21. Correlation between biochemical glycogen concentration and

TEM-based glycogen particle volume .....................................113

Figure 22. Summary of the experimental design ...................................119

Figure 23. Differences in single particle volume between subcellular locations ....130

Figure 24 (A). Frequency distribution for the single particle diameters .................131

Figure 24 (B). Frequency distribution for the single particle diameters per fibre type .132 
Figure 25. Differences in glycogen volume between subcellular locations 134

Figure 26. Effect of time post-exercise on total myofibrillar glycogen concentration, number of glycogen particles, and single glycogen particle size

Figure 27. Effect of time post-exercise on the net rates of glycogen synthesis ...152

Figure 28. Relative frequency distribution of single particle diameter $(\mathrm{nm})$ at the four different times post-exhaustion $(0,4,24$, and $48 \mathrm{~h})$ .154

Figure 29 (A). Effect of subcellular locations on glycogen concentration 156

Figure 29 (B). Effect of subcellular locations on single glycogen particle size

Figure 30. Effect of time and subcellular location on the net rate of single glycogen particle volume synthesis

Figure 31. Illustration of muscle tissue at $0,4,24$ and $48 \mathrm{~h}$ post-exercise .161

Figure 32. Effect of exercise on glycogen concentrations

Figure 33 (A). Pre-exercise relative frequency distribution of single particle diameter per subcellular locations

Figure $33(B)$. Post-exercise relative frequency distribution of single particle diameter per subcellular locations

Figure 34. Effect of fibre type on single particle volume

Figure 35 (A). Pre- and post-exercise absolute frequency distribution of glycogen particles, as measured per $\mu \mathrm{m}^{3}$ of muscle tissue

Figure 35 (B). Pre- and post-exercise relative frequency distribution of single particle diameter 190

Figure 36. Number of glucosyl units per tier and a glycogen particle .212

Figure 37. Relative frequency distribution of single particle combined with the corresponding number of glucosyl units per tier .214

Figure 38. Comparison of the frequency distribution of single particie diameter of a McArdle's patient and a glycogen-loaded control subject .216

Figure 39 (A). Illustrations of the possible mechanisms to explain the relative increase in frequency distribution of intermediate-size glycogen particles .228

Figure 39 (B). Iliustrations of the possible mechanisms to explain the relative decrease in frequency distribution of larger glycogen particles .229

Figure 39 (C). Illustrations of the possible mechanisms to explain the lack of 
change in the relative frequency distribution of smaller glycogen particles

Figure 39 (D). Sum of mechanisms most likely involved in the pre-to post-exercise changes in relative frequency distribution reported in Chapter VII ......233

Figure 40. Pre- to post-exercise percentage of decrease in particle number per particle diameter 234 
Chapter I

General Thesis Introduction 


\section{Introduction}

Glycogen is a branched polymer of glucose. In humans, it is principally stored in liver and skeletal muscles and the latter provides the largest storage form of glucose. In addition, skeletal muscle glycogen is known to play an important role in whole body glucose homeostasis. Defects in skeletal muscle glycogen metabolism are known to be associated with a variety of metabolic disorders including obesity, insulin resistance and Syndrome $X$ as well as diabetes mellitus. The physiological importance of skeletal muscle glycogen in proper body function has stimulated extensive research in this area. Accordingly, numerous and extensive biochemical experiments have provided the basis of our understanding of glycogen metabolism over the past 150 years. However, a wide range of research questions can not be addressed using biochemical technology, which can solely determine total skeletal muscle or single fibre glycogen concentrations.

Direct visualisation using transmission electron microscopy provides the unique ability to investigate subcellular compartmentalisation, as well as to examine the pattern of glycogen distribution with respect to glycogen particle size and numbers. The importance of this aspect of glycogen metabolism is becoming increasingly recognised with the emerging concept of the subcellular organisation of the enzymatic machinery and metabolic pathways involved in glycogen metabolism. However, the information available in this area of glycogen metabolism is limited to a few qualitative investigations. Therefore, the need for an objective analysis of the subcellular distribution of human skeletal muscle glycogen is obvious. Such information will provide clues to understanding the role of the subcellular compartmentalisation pattern of glycogen as a potential regulatory control mechanism in the metabolism of carbohydrate in human skeletal muscles. The present thesis provides the first quantitative analysis of the subcellular distribution of human skeletal muscle glycogen. 
Chapter II

Literature Review 


\section{Skeletal muscle glycogen structure and function}

Structure.

Glycogen is a branched polymer of glucose. While much is left to be clarified, a substantial amount of information is available concerning the branching structure of glycogen (for review see Roach et al. 2001). The group of Whelan and co-workers have proposed a model for the structure of glycogen (Gunja-Smith et al. 1970) which has been widely accepted and refined (Goldsmith et al. 1982; Meléndez-Hevia et al. 1993).

Figure 1 illustrates this model as adapted from Meléndez and co-workers (1999).

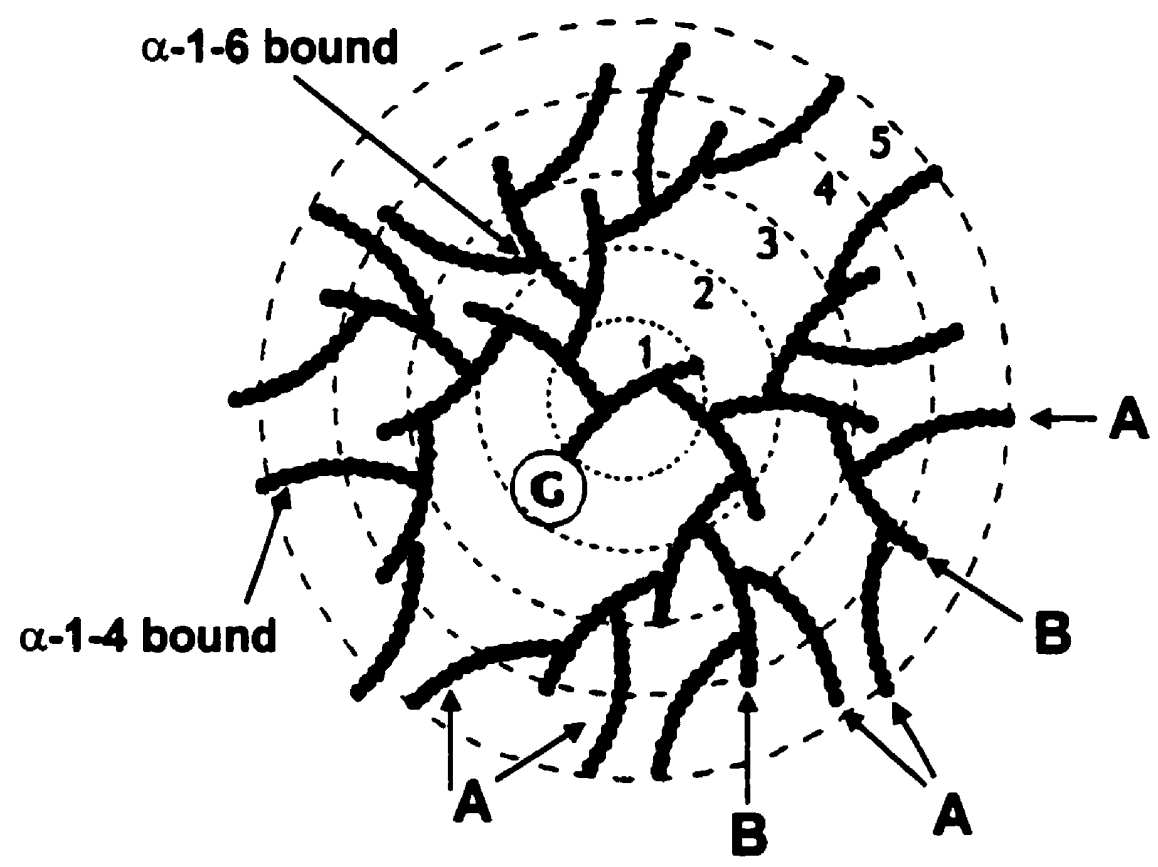

Figure 1. Glycogen structure according to Whelan's model (adapted from Meléndez et al. 1999). Glycogen is a spherical particle built in concentric tiers ( 5 are shown here). It is formed by unbranched (A) and branched (B) chains of glucose polymerised by $\alpha-1-4$ glycosidic bonds. Each "B" chain has two branching points linked by $\alpha-1-6$ glycosidic bonds. All "A" chains are found exclusively in the last available tier. The protein core is the self-glucosylating protein glycogenin (G). 
The Whelan model proposes that glycogen has a regular spherical structure organised into concentric tiers around a protein core; that it holds approximately equal numbers of " $A$ " chains (unbranched chains) and " $B$ " chains (branched chains); and that all " $A$ " and " $B$ " chains are of uniform lengths (Goldsmith et al. 1982). The " $A$ " chains, being unbranched, are found exclusively in the outermost portion of the glycogen particle. The " $B$ " chains are branched on average on two positions (glucose residues 56 and 9-10) and they are on opposite sides of the spiral of the original chain (Goldsmith et al. 1982). $\alpha-1-4-$ glucosidic bonds link the glucose residues in a straight chain and represent more than $90 \%$ of all bonds in a glycogen particle. $\alpha-1-6-$ glucosidic bonds provide the branching pattern and represent less than $10 \%$ of all the bonds in a glycogen particle (Chapter 5, Newsholme and Leech 1983). The organisation into concentric tiers represents layers of branched chains enclosing one another and adding up to a three dimensional structure as a sphere (Figure 1). The three dimensional structure arises from the nature of the $\alpha-1-6-$ glucosidic bonds described above.

Based on this model and on the molecular weight of the $\beta$-particle of rabbit muscle glycogen (10 Da) as stated by Wanson and Drochmans (1968), Goldsmith and colleagues (1982) reported the following structural parameters for a mature glycogen $\beta$ particle:

\begin{tabular}{|l|l|}
\hline $\begin{array}{l}\text { Molecular weight of rabbit muscle } \beta \text {-particle } \\
\text { (Reported by Wanson and Drochmans (1968)) }\end{array}$ & $10^{7} \mathrm{Da}$ \\
\hline Total number of tiers & 12 \\
\hline Total number of glucose residues & 55000 \\
\hline Average chain length & 13 glucose residues \\
\hline $\begin{array}{l}\text { Effective length per tier } \\
\text { (every tier is the same thickness) }\end{array}$ & $1.9 \mathrm{~nm} \times 2=\sim 3.8 \mathrm{~nm}$ \\
\hline Total diameter of the particle & $3.8 \mathrm{~nm} \times 11$ tiers $=\sim 42 \mathrm{~nm}$ \\
\hline
\end{tabular}

Finally, owing to this organisation and the degree of branching, the number of chains in any tier is twice that of the previous one (Meléndez-Hevia et al. 1993). Taken 
together, this information suggest that the amount of glucose directly available to be released by glycogen phosphorylase (the enzyme responsible for cleaving the $\alpha-1-4$ glucosidic bonds) is $34.6 \%$ of the total glucose contained in the particle, independent of the number of tiers of the particle. Therefore, in a mature $\beta$-particle containing a total of 55000 glucose residues, one can predict that approximately $19000(\sim 35 \%$ of 55000$)$ will be present in the $12^{\text {th }}$ tier, $12400(-23 \%$ of 55000$)$ in the $11^{\text {th }}$ tier, $8100(\sim 15 \%$ of $55000)$ in the $10^{\text {th }}$ tier, and $5300(\sim 10 \%$ of 55000$)$ in the $9^{\text {th }}$ tier. Accordingly, only approximately $18 \%$ of the total glucose of a mature $\beta$-particle is located in tiers 8 to 1 . The physiological consequences of this will be addressed later.

It is generally accepted that size limit is determined by the density of the last $\left(12^{\text {th }}\right)$ tier (Goldsmith et al. 1982). Meléndez and co-workers (1997) reported that in the $12^{\text {th }}$ tier, glucose occupies $26 \%$ of the space while in a hypothetical $13^{\text {th }}$ tier, the space occupied by glucose would be $62 \%$. Because the enzymes involved in glycogen metabolism also occupy space, the density reached in the $13^{\text {th }}$ tier would be such that the enzymes would have no room left to attach to the molecule and continue working.

To conclude, in this model, the most mature form of glycogen is structured as a spherical particle consisting of 11 successive tiers of " $B$ " chains surrounded by a $12^{\text {th }}$ tier containing " $A$ " chains, all chains averaging 13 glucose residues, for a total of 55000 glucose residues. The model predicts that $34.6 \%$ of the glucose residues are immediately available in every outer tier, independent of the number of tier, and has been demonstrated to achieve an optimised molecule through the optimisation of each intermediate step involved in its metabolism (Meléndez et al. 1997). 
Function.

While it is highly likely that all the functions of glycogen have not yet been discovered, the two main recognised roles of glycogen in the organism are to provide a storage form of glucose and to play a role in whole body glucose homeostasis (Roach et al. 2001).

Although most cells in the human body do contain some storage form of glucose, the two main tissues involved in glucose storage are the liver and the skeletal muscles. The glycogen stored in the liver is very concentrated, but its absolute content of $90 \mathrm{~g}$ is small relative to needs of the organism and to the approximate $350-400 \mathrm{~g}$ of glycogen stored in skeletal muscles (Chapter 8, Newsholme and Leech 1983). Therefore, more than $80 \%$ of the total body carbohydrate $(\mathrm{CHO})$ is located in skeletal muscles. The structure of glycogen described above allows for the storage of large amounts of glucose with a minimal osmolarity (Roach et al. 2001) and a maximal accessibility at any stage of its degradation (Meléndez et al. 1997).

While skeletal muscle glycogen is used primarily to provide energy to the contractile apparatus, liver glycogen is used primarily for maintaining a stable blood glucose level. A remarkable feature of liver metabolism is its ability to change rapidly from an organ of blood glucose storage to an organ that produces glucose to be released into the blood (for review see Roach et al. 2001). This major difference in $\mathrm{CHO}$ metabolism between the two tissues is mainly due to the absence of glucose-6phosphatase or its equivalent in skeletal muscles (Connett and Sahlin 1996). This liver enzyme is responsible for cleaving the phosphate group on a glucose molecule, hence allowing its exit from the hepatocytes. Therefore, the majority of the skeletal muscle glycogen (i.e. all the glucose-1-phosphate released by the enzyme glycogen phosphorylase) can not be released into the blood and must be used for energy 
production within the muscle or for re-storage as glycogen.

In line with its main function, liver glycogen demonstrates a wide diurnal variation and a high dependence on dietary $\mathrm{CHO}$ (Chapter 8, Newsholme and Leech 1983). In fact, in a series of classical experiments, Hultman and colleagues observed that the liver is practically depleted of its CHO content in as little as 24 hours of fasting (Nilsson and Hultman 1973). In contrast, four days of total starvation decreased the glycogen levels by only $40 \%$ in resting skeletal muscles (Hultman and Bergstrom 1967). Therefore, whereas liver glycogen is largely dependent on the nutritional state of an individual, skeletal muscle glycogen is more refractory to dietary manipulations and can be metabolised effectively by muscular contractions (Roach et al. 2001).

Muscle glycogen synthesis also plays a key role in blood glucose homeostasis (Kruszynska et al. 2001). Indeed, skeletal muscle glycogen synthesis has been shown to represent the major metabolic pathway for glucose disposal in both normal and diabetic subjects (Shulman et al. 1990). Shulman and co-workers (1990) reported that approximately $80 \%$ of post-prandial glucose, insulin-stimulated uptake, is stored as glycogen in skeletal muscle. Furthermore, they found a diminished ability to synthesize skeletal muscle glycogen in most patients with type II diabetes, and implied that this defect is likely to play a dominant role in the insulin resistance that occurs in those patients. The aforementioned functions emphasize the importance of skeletal muscle glycogen in whole body glucose homeostasis.

\section{Glucose disposal and glycogen metabolism in skeletal muscles}

It is beyond the scope and focus of this review to provide a detailed discussion of the characteristics of each of the pathways and enzymes involved in glucose disposal and glycogen metabolism (for a complete recent review see Roach et al. 2001). 
Glucose disposal first involves the transport of glucose through the cellular membrane and its phosphorylation upon entering the cell. Subsequently, glucose can either be oxidised to $\mathrm{CO}_{2}$, converted to lactate, or stored as muscle glycogen. A brief overview of the pathways involved is necessary to facilitate an understanding of the material presented in the present thesis.

\section{Glucose transport.}

The first step in glucose disposal involves the transmembrane transport of glucose from the blood to the interior of the skeletal muscle fibre, where skeletal muscle glycogen synthesis takes place. Whereas glucose is suspected to move between the blood plasma and the interstitial fluid by simple diffusion (Zierler 1999), its transport across the muscle cell membrane (sarcolemma and t-tubular system) is known to occur by facilitated diffusion by way of glucose transporters (for review see Zierler 1999). It is worth noting that both simple and facilitated diffusion require a favourable glucose concentration gradient. However, while under most physiological conditions the interstitial glucose concentration is believed to be similar to the venous plasma concentration ( $\sim \mathrm{mM})$, most evidence points to the likelihood that, over a wide range of normal blood glucose concentrations, if there is any free glucose in skeletal muscle cells, it is below the resolving power of currently available methods (Richter 1996; Zieler 1999). Therefore, a highly favourable concentration gradient appears to be present under most experimental conditions.

Although a total of six functional glucose transporter (GLUT) isoforms has been identified (Zierler 1999), GLUT-1 and GLUT-4 are the two isoforms present in skeletal muscles (Douen et al. 1990). GLUT-1 is expressed in most cells, and appears to reside primarily in the sarcolemma where it is believed to participate mainly in basal glucose transport (Douen et al. 1990). GLUT-4, on the other hand, is found exclusively in 
insulin-sensitive tissues, including skeletal and cardiac muscle and adipose tissue (Birnbaum 1992). Insulin is a pancreatic hormone responsible for the maintenance of blood glucose levels in a narrow physiological range. Under basal conditions, most of the GLUT-4 is found laced in the wall of intracellular vesicles (Klip et al. 1996). When stimulated by either insulin (Lund et al. 1993) or muscular contractions (Cartee et al. 1989), these vesicles are mobilised to fuse with the cell surface membrane, increasing the density of plasma membrane GLUT-4, and consequently, glucose transport. Furthermore, it has been shown that the majority of this translocation occurs at the level of the transverse-tubules, not the sarcolemma, suggesting an important role of the transverse-tubule membrane system in delivering nutrients to the centre of skeletal muscles (Dohm and Dudek 1998). Most reports show that the degree of increase in skeletal muscle glucose uptake induced by insulin or exercise exceeds the degree of increase in plasma membrane GLUT-4, implying that there may be an effect of these stimuli on transport efficiency as well as on transporter availability (Zierler 1999). Therefore, the increase in glucose uptake induced by insulin and exercise is likely accounted for in part by an increase in GLUT density on the cell surface, and in part by increased effectiveness of GLUT-4 (Zierler 1999). In a recent experiment, Kraniou and co-workers (2000) reported that a single bout of moderate intensity exercise for 60 minutes was sufficient to increase GLUT-4 mRNA, implying a role for contraction in stimulating GLUT-4 protein expression as well as GLUT-4 translocation.

Interestingly, much evidence exists to suggest that these stimuli (insulin and contraction) activate glucose transport by different mechanisms (Ivy and Kuo 1998). In fact, two separate intracellular GLUT-4 pools have now been identified, one responding to insulin and the other to contraction (Coderre et al. 1995). Taken together, these findings demonstrate the important role of insulin (post-prandial) and exercise (i.e. contraction) in regulating glucose uptake in skeletal muscles. 
Interestingly, both the insulin- and contraction-stimulated pathways for muscle glucose transport activation have recently been shown to be dependent on glycogen concentration (Derave et al. 1999; Kawanaka et al. 1999 and 2000). In these studies, it was shown that increasing glycogen concentration in skeletal muscle significantly decreased both insulin- and contraction-stimulated glucose transport, and that this decrease was entirely explained by translocation of fewer GLUT-4 transporters to the cell surface. While the molecular mechanism by which glycogen level influences GLUT4 translocation is still unclear (Kawanaka et al. 2000), it clearly represents a means of controlling glycogen metabolism in skeletal muscle.

\section{Glucose phosphorylation.}

Upon entering the skeletal muscle cell, glucose is rapidly and irreversibly phosphorylated to glucose-6-phosphate (G-6-P) by the enzyme hexokinase II (Newgard et al. 2000). As mentioned above, the reverse reaction is not possible in skeletal muscle as the enzyme G-6-phosphatase is lacking. Hexokinase II is allosterically inhibited by G6-P and therefore, any accumulation of G-6-P, as a result of a block in the possible pathways of glucose disposal (glycogen synthesis or glycolysis), will inhibit hexokinase activity. This in turn, could lead to an accumulation of glucose in the muscle cell, and decrease the glucose concentration gradient necessary for glucose transport across the sarcolemma and transverse-tubule. Interestingly, hexokinase II has been reported to be most active when bound to the mitochondria, allowing direct access to ATP (Wilson 1994). The liver equivalent to hexokinase II, glucokinase, has also been shown to translocate between the cytoplasm and the nucleus in response to changes in glucose concentration (Brown et al. 1997). Briefly, at low glucose concentration, glucokinase appears anchored to the nucleus by an inhibitory protein; this in turns keeps glucokinase in an inactive state. At high glucose concentration, glucokinase translocates from the 
nucleus to the cytoplasm, where it is no longer inhibited by the regulatory protein. As pointed out by Newgard and colleagues (2000), this discrete localisation of glucose phosphorylating enzymes in muscle and liver represents another potential mechanism for controlling the flux of G-6-P into the glycogen metabolic pathways. Interestingly, and in line with what has been observed for GLUT-4, a single bout of exercise has been shown to increase gene expression of hexokinase II (Koval et al. 1998; Kraniou et al. 2000), implying an additional mechanism by which muscle contraction increases glucose disposal.

Finally, for glucose to be incorporated into glycogen, G-6-P is first converted to glucose-1-phosphate (G-1-P) and uridine diphosphate-glucose (UDPG) in a sequence of reactions catalysed by phosphoglucomutase and UDPG pyrophosphorylase (Azpiazu et al. 2000). Both latter steps are not thought to be rate limiting in glucose disposal (Richter 1996).

Glycogen synthesis.

Glycogenin. The first phase of glycogen synthesis is mediated by the selfglucosylating protein glycogenin (for review see Roach and Skurat 1997). The protein was first identified and characterised by Kennedy and co-workers in 1985. Mammalian glycogenin has a subunit molecular weight $\left(M_{r}\right)$ of $\sim 37000 \mathrm{Da}$. It behaves as a dimer but there is evidence that it might associate as a tetramer (Lomako et al. 1988). Three essential properties of glycogenin make it indispensable in the initiation of glycogen biosynthesis. The first one involves its ability to self-glucosylate, using UDPG as the glucose donor, by forming a linkage between glucose and tyrosine ${ }^{194}$ (Smythe et al. 1988). Secondly, glycogenin is capable of further promoting self-glucosylation by the formation of $\alpha-1-4$-glucosidic bonds (figure 1), until a chain of 8 to 12 glucose residues is formed. Finally, glycogenin has the ability to serve as a substrate for further elongation 
by glycogen synthase (Roach et al. 2001). Interestingly, in rabbit skeletal muscles, glycogenin has been found to be covalently bound to glycogen synthase in a 1:1 molar ratio (Pitcher et al. 1987). However, whether this association still holds true in vivo on a growing glycogen particle is not known (Roach et al. 2001). In the liver, the ratio of glycogenin to glycogen synthase has been reported to be much lower compared to skeletal muscle (Smythe et al. 1989), suggesting a different organisation in that organ.

Additional critical information was provided by Smythe et al. (1990), who demonstrated that, under most normal conditions, a deglucosylated form of glycogenin is not present in skeletal muscle, suggesting that glycogenin might have to be synthesised on demand. This may constitute an additional mechanism for controlling glycogenesis. Indeed, recent evidence shows skeletal muscle glycogenin mRNA to increase by more than two-fold only three hours following 60 minutes of moderate intensity exercise (Kraniou et al. 2000), a situation in which glycogen synthesis is greatly enhanced. Although it is still unclear whether this increase leads to an immediate expression of glycogenin protein, Shearer and co-workers (unpublished data) recently provided evidence to demonstrate that, along with the increase in glycogenin mRNA, glycogenin activity was also increased within 30 minutes post-exercise.

To evaluate a possible role for glycogenin in regulating glycogen synthesis, independent laboratories have over-expressed glycogenin in different cells (Hansen et al. 1998; Skurat et al. 1997). Hansen and co-workers (1998) were able to show that glycogenin over-expression in L6 myoblasts leads to a modest increase in glycogen concentration. Furthermore, they found this increase to be exclusively in the smallest glycogen species (to be described later). While Skurat and colleagues (1997) were not able to demonstrate that glycogenin over-expression in rat fibroblasts enhances glycogen content, their findings agreed with that of Hansen and co-workers (1998) in that it leads to the production of additional, smaller, glycogen particles. Interestingly, a 
recent study by Shearer and colleagues (2000) demonstrated that glycogenin activity is proportional to muscle glycogen concentration, providing further evidence to support a role of glycogenin as a regulator of glycogen synthesis. Further studies are required to clarify the role of glycogenin in the control of glycogen synthesis.

The regulation of glycogenin activity is not clearly understood. Several mechanisms have been proposed including its regulation by phosphorylation (Lomako and Whelan 1988), by changes in glucose concentration (Cao et al. 1993) and by interaction with other proteins (Banqué et al. 1997; Cao et al. 1993). However, unequivocal evidence is lacking to substantiate these notions. Similarly, evidence for a control of gene expression or of glycogenin activity is missing (Roach et al. 2001). Future research should clarify these unanswered questions.

Glycogen synthase. The next step in glycogen synthesis involves the incorporation of additional UDPG into the non-reducing ends of glucosylated glycogenin or other preexisting glycogen particles. The enzyme responsible for catalysing this formation of additional $\alpha-1-4-g$ lucosidic bonds is glycogen synthase. Glycogen synthase (GS) from skeletal muscle is a tetramer of four identical 84000 Da subunits (Kruszynska et al. 2001). The control of GS has been studied extensively (for review see Cohen 1986; Roach 1990; Roach et al. 2001). GS control is complex and is accomplished by a variety of mechanisms, which will be briefly described here.

Covalent modification by phosphorylation of the enzyme has been found to greatly affect the activity of GS (Cohen 1986). The enzyme is phosphorylated on up to nine residues by as many as seven different kinases (Kruszynska et al. 2001), resulting in its progressive inactivation. This multiple phosphorylation of GS has been demonstrated to occur in a hierarchical fashion (Roach 1990). In contrast, dephosphorylation of GS leads to its activation. The current consensus is that the 
glycogen associated protein phosphatase (PP1) accounts for the majority of GS dephosphorylation in skeletal muscle (Cohen 1986). In fact, it is believed that PP1 is responsible for dephosphorylating GP and phosphorylase kinase as well as GS (Roach et al. 2001). The skeletal muscle glycogen associated form of PP1 is composed of a type 1 protein phosphatase catalytic subunit (PP1C), and a regulatory subunit that is thought to act as a targeting subunit (Hubbart and Cohen 1993). The physiological consequences of these interactions on glycogen metabolism will be addressed in the section "Glycosomes - the organelles of glycogen metabolism".

A number of metabolites have been shown to affect GS activity by allosteric modulation (Piras et al. 1968). However, the most important metabolite is probably G-6$P$, which is a powerful allosteric activator that has been found to override the inhibition caused by phosphorylation (Jensen et al. 2000). Furthermore, the binding of G-6-P to GS induces a conformational change, which increases GS susceptibility to dephosphorylation by PP1 (Cadefau et al. 1997). The net activity of GS thus appears to depend on both the degree of phosphorylation and the G-6-P concentration (Piras et al. 1968).

While a similar phenomenon has been involved in the regulation of glucokinase, an emerging concept in the area of GS activity control is the GS translocation to different subcellular locations in response to various stimuli. GS translocation has been demonstrated in 3T3-L1 adipocytes (Brady et al. 1999; Jensen et al. 2000), isolated rat hepatocytes (Fernandez-Novell et al. 1997), cultured rat hepatocytes (Garcia-Rocha et al. 2001) and rat 1 fibroblasts expressing rabbit muscle glycogenin (Skurat et al. 1997). In a recent report, the phenomenon of GS translocation was also observed in rat skeletal muscles (Nielsen et al. 2001). As is the case for glucokinase and the glucose transporter system, the translocation of GS to different subcellular locations could 
represent a means of controlling the rate and extent of glycogen synthesis in the different cellular compartments.

Insulin, in addition to its ability to increase glucose transport, has long been shown to independently activate GS (Cohen 1986). Although the precise signalling pathways used by insulin to activate GS remain unclear, insulin is known to utilise all three mechanisms described above to stimulate GS (Jensen et al. 2000). On the other hand, epinephrine is known to result in a decreased GS activity (Raz et al. 1991). This effect is believed to be partly linked to the increased peripheral insulin resistance induced by epinephrine (Deibert et al. 1980), and partly linked to the effect of epinephrine on the kinases responsible for phosphorylating GS (Connett and Sahlin 1996).

Muscle contraction is recognised for its ability to increase GS activity (Connett and Sahlin 1996; Nielson et al. 2001). While the mechanisms are unclear, this increase in GS activity during and following exercise has been hypothesised to be linked to the exercise associated low glycogen concentration, increased insulin sensitivity and the increased G-6-P levels (Connett and Sahlin 1996). Furthermore, the pattern of muscle glycogen synthesis following its depletion by exercise has been shown to be biphasic, with an initial rapid insulin-independent period of synthesis, followed by a slower insulin dependent rate of synthesis (for review see Ivy and Kuo 1998). Price and co-workers (1994) demonstrated that the transition between the two phases was dependent on the muscle glycogen level, with the initial insulin-independent phase only occurring upon glycogen levels smaller than $\sim 100-125 \mathrm{mmol} / \mathrm{kg}$ of dry muscle weight.

In line with those findings, besides its effect on GLUT-4 translocation, the intramuscular glycogen concentration has consistently been demonstrated to exert a powerful regulatory effect on glycogen synthesis (Laurent et al. 2000; Montell et al. 1999; Munger et al. 1993; Price et al. 2000; Vandenberghe et al. 1999). However, 
limited information is available regarding the mechanisms by which glycogen levels feedback to regulate glucose transport and glycogen metabolism. From a theoretical point of view, glycogen could limit its own synthesis by feedback inhibition of glucose transport, hexokinase, or GS activity or by activating glycogenolysis (Laurent 2000). Indeed, Nielsen and co-workers (2001) recently performed an elaborate experiment to provide some insights into potential mechanisms. They found that GS translocates from a glycogen-enriched membrane fraction to a cytoskeleton fraction when glycogen levels are decreased, and hence, that GS subcellular location is influenced by muscle glycogen content. Furthermore, they demonstrated that not only basal, but also insulin- and contraction-stimulated GS activity is strongly regulated by glycogen content in skeletal muscles, with the contraction-induced increase in GS activity being solely a result of a decrease in muscle glycogen content. Finally, they suggested that glycogen is a more potent regulator of GS activity than is insulin. Taken together, these findings provide evidence to clarify the mechanism by which muscle contraction induces an increase in GS activity both during and following exercise, and confirm previous findings that glycogen regulates its own synthesis by acting on GS activity (Laurent et al. 2000; Montell et al. 1999; Munger et al. 1993).

The concept discussed in the present section provides further evidence for the importance of subcellular compartmentalisation in the regulation of glycogen metabolism. This intra-cellular translocation of some of the key enzymes involved in glycogen metabolism, including GS and glucokinase, as well as of the glucose transporter system, could be regulated by local glycogen concentrations. Once again, however, the exact mechanism by which the glycogen level induces translocation is unknown.

To summarise, GS activity is controlled by any one of three main mechanisms: (1) covalent phosphorylation; (2) non-covalent allosteric modification (mainly G-6-P); and 
(3) translocation to different $s$ bcellular compartments. Insulin and intra-muscular glycogen levels can influence GS activity, and thus glycogen synthesis, by any of the above mentioned mechanisms.

Branching enzyme. The branching enzyme (BE) catalyses the last step in glycogen biosynthesis, introducing $\alpha-1-6$ branch points into glycogen molecules (Caudwell and Cohen 1980). BE has a $\mathrm{M}_{\mathrm{r}}$ of $77000 \mathrm{Da}$, and is believed to act as a monomeric protein (Caudwell and Cohen 1980). BE works together with GS during glycogen synthesis. When GS has promoted the growth of a chain to at least 11 glucose residues linked by $\alpha-1-4$ bonds, BE transfers a set of 7 glucose residues to another chain making $\alpha-1-6$ bonds between glucoses, and producing new branches (Meléndez et al. 1999). Under normal conditions, this is not believed to be rate limiting in glycogen biosynthesis (Caudwell and Cohen 1980; Meléndez et al. 1997). Interestingly, Meléndez and colleagues (1997) provided evidence to suggest that it is the ratio of activities between $B E$ and GS that accounts for the control of the glycogen chain length (on average 13 glucose residues per chain).

Model for glycogen synthesis. Based on the properties of the enzymes described above, a model for glycogen biosynthesis has been proposed by Roach and co-workers (Roach and Skurat 1997). Figure 2 illustrates this model, in which the synthesis of glycogen involves minimally three definable steps: initiation, elongation, and branching (Roach et al. 2001).

The initiation step involves self-glucosylation by glycogenin. As described above, the self-glucosylation reaction appears to involve two different types of chemical reaction, first the formation of a tyrosyl linkage to a specific tyrosine residue in the 
protein, and then elongation via the formation of a $\alpha-1-4-$ glycosidic linkages (Roach and Skurat 1997). This oligosaccharide primer is extended to 8-12 residues, at which point GS and BE take over the synthesis of glycogen by elongation and branching, respectively (Roach et al. 1998). While many aspects of glycogen synthesis remain unknown, this model has gained general acceptance.

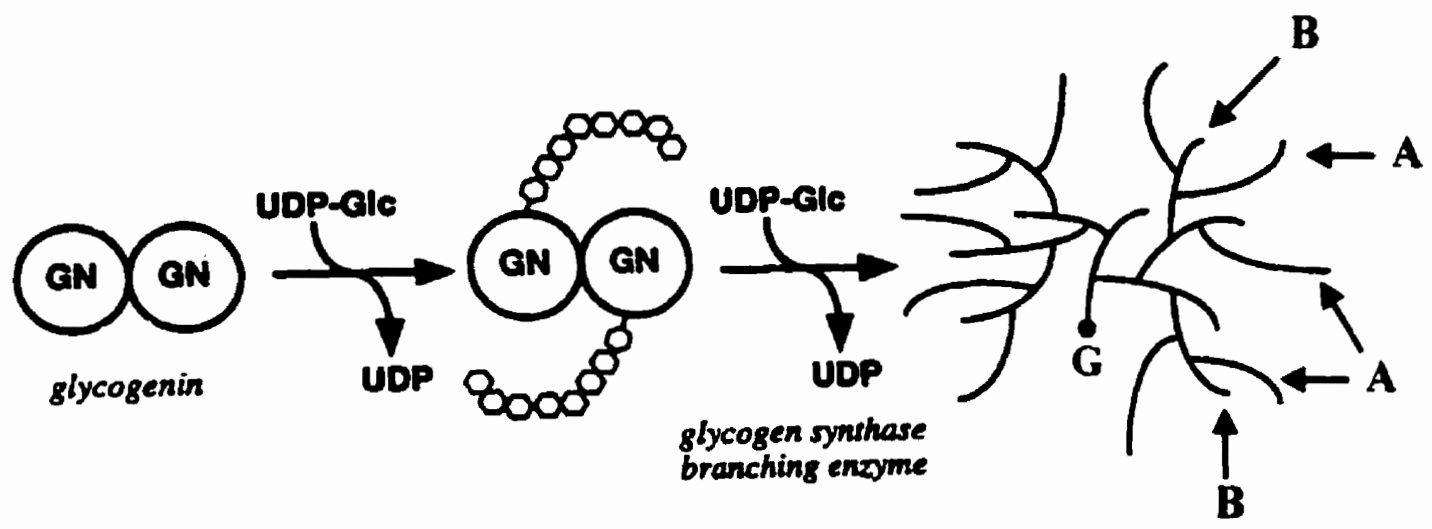

Figure 2. Pathway of glycogen biogenesis as proposed by Roach and colleagues (adapted from Roach et al. 2001). The initiation step, mediated by glycogenin (GN), serves to generate an oligosaccharide that acts as a substrate for the subsequent steps involving elongation and branching by the action of glycogen synthase and branching enzyme, respectively. " $A$ " and " $B$ " represent unbranched and branched chains of glucose residues, respectively, as described in the section "Skeletal muscle glycogen structure and function".

In 1991, the existence of a defined, stable intermediate in the synthesis and degradation of mature glycogen was proposed (Lomako et al. 1991). This stable intermediate was termed pro-glycogen (PG) (Lomako et al. 1991). PG was defined as being a 400000 Da species of glycogen that is insoluble in trichloroacetic acid (TCA) due to its higher proportion of protein relative to carbohydrates (Lomako et al. 1991). Furthermore, it was proposed that a separate "PG synthase" exists to mediate the 
synthesis of PG from glycogenin since it can occur at micro-molar UDPG concentrations, levels well below the UDPG level required for the "classical" GS (Lomako et al. 1993). Finally, it was suggested that PG and mature or macro-glycogen (MG) may be metabolised as separate entities (for review see Alonso et al. 1995). However, the existence of PG as a stable and discrete intermediate in glycogen synthesis has been questioned (Skurat et al. 1997). Skurat and colleagues (1997) proposed that the finding of PG as a stable intermediate in fact represents an artefact caused by the use of discontinuous electrophoresis. In fact, they were able to reproduce the results from Lomako and colleagues when using the same method. However, they also used SDS / PAGE in a continuous buffer system and revealed that this product of $C^{14}$ glucose-labelled was not a discrete entity (Skurat et al. 1997). Rather, they showed that glycogen particles exist as a continuum of glycogenin-containing species from low molecular mass to sizes significantly greater than $400 \mathrm{KDa}$, hence disproving the presence of the accumulation of any major intermediate form of glycogen particles. According to their findings, PG would simply represent all glycogen intermediates with $M_{r}$ ranging from glycogenin to $400000 \mathrm{Da}$, while MG would represent all glycogen intermediates with $M_{r}$ ranging from 400000 Da to the mature glycogen molecule of $10^{7}$ Da.

Rate determining reaction in glycogen synthesis and glucose disposal. As mentioned in a previous section, most of the glucose that is removed from the blood after a meal is converted into skeletal muscle glycogen (Shulman et al. 1990). However, the relative importance of glucose transport and glycogen synthase in controlling the rate of glucose disposal and glycogen synthesis has been debated over the years. While the rate limiting reaction for glycogenesis has in the past been considered to be the transfer of glucose from UDPG to glycogen (Ivy and Kuo 1998), recent evidence strongly supports 
a shared control between glucose transport and GS activity (Azpiazu et al. 2000; Jucker et al. 1999; Schulz 1998). Schulz (1998) performed a theoretical analysis of glycogen metabolism using metabolic control analysis. His conclusions were that the control of glycogen synthesis is not accomplished by any rigid parameter of any of the components of the glycogen metabolic system, but rather that the distribution of control is flexible and shifts according to different physiological and biochemical conditions (Schulz 1998). More recently, Jucker and co-workers (1999) used a similar approach to calculate the distributed control of insulin-stimulated skeletal muscle glucose disposal in rats during hyperinsulinemic clamp. Their results showed that glucose transport / phosphorylation and GS could account for 55 and $30 \%$ of the control of glucose disposal, respectively. Taken together, these results are consistent with the view that the control of glucose disposal and glycogen synthesis involves both glucose transport and GS activity (Azpiazu et al. 2000), and that their relative importance may vary with specific physiological situations (Roach et al. 2001).

\section{Glycogenolysis.}

Muscle glycogen is a key substrate for the generation of energy during contractile activity. In fact, skeletal muscle glycogen is the major $\mathrm{CHO}$ substrate during exercise and its rate of degradation is primarily dependent upon exercise intensity (Vollestad and Blom 1985). The key enzymes involved in glycogenolysis are glycogen phosphorylase (GP) and debranching enzyme. They will be briefly described here.

Glycogen phosphorylase and debranching enzyme. $\quad$ GP is the rate-limiting enzyme catalysing the first step in the intra-cellular degradation of glycogen. GP catalyses the phosphorolysis of the $\alpha-1-4$-glucosidic bonds to generate G-1-P (for review see Johnson 1992; Madsen 1986). Working together with GP, debranching enzyme, a 
monomer with $M_{r}$ of $164000 \mathrm{Da}$, is the enzyme responsible for eliminating the $\alpha-1-6$ branched points, thus allowing the continued release of G-1-P by GP (Taylor et al. 1975). There is little evidence for the debranching enzyme to have any regulatory properties, and it is not generally considered to be rate limiting for glycogenolysis (Roach et al. 2001). GP was the first enzyme shown to exist in oligomeric form and to be metabolically interconverted between a less active " $b$ " form and a more active " $a$ " form (Keller and Cori 1953). GPa and GPb have been shown to be a tetramer and a dimer of identical subunits, respectively (Madsen and Cori 1956). Each monomer yields a $M_{r}$ of $\sim 97000 \mathrm{Da}$, and the conversion from the GPb to the GPa form is accomplished by phosphorylation (Madsen 1986). In contrast to GS, GP contains a single phosphorylation site, which is modified by a single kinase (Roach et al. 2001).

Phosphorylase kinase is the enzyme responsible for phosphorylating GP (Madsen 1986). Phosphorylase kinase plays a central role in the regulation of glycogen metabolism, mediating the phosphorylation (i.e. activation) of GP and the partial phosphorylation (i.e. deactivation) of GS, thus shifting the balance toward glycogenolysis (Kruszynska et al. 2001). Phosphorylase kinase is a large multi-subunit protein, with a $\mathrm{M}_{\mathrm{r}}$ of approximately $1.2 \times 10^{6} \mathrm{Da}$ (for review see Pickett-Gies and Walsh 1986). Phosphorylase kinase requires $\mathrm{Ca}^{2+}$ for activity, and its activity is also increased by phosphorylation (Pickett-Gies and Walsh 1986). The protein phosphatase thought to dephosphorylate both GP and phosphorylase kinase is protein phosphatase 1, the same enzyme responsible for the activation of GS (Roach et al. 2001). The hormones insulin and epinephrine are also known to influence the activity of GP by modulating its phosphorylation state. Epinephrine indirectly activates phosphorylase kinase by stimulating its phosphorylation by CAMP protein kinase, thus increasing the phosphorylation (i.e. activation) of GP by phosphorylase kinase (Connett and Sahlin 1996). In contrast, insulin activates PP1, hence causing the deactivation of GP by 
simultaneously dephosphorylating GP directly and by dephosphorylating (i.e. deactivating) phosphorylase kinase (Connett and Sahlin 1996). This integrated control system of glycogen metabolising enzymes illustrates well the complex interactions involved, as well as the need for considering all components in order to obtain a complete understanding of glycogen metabolism.

For many years, GPb to GPa transformation was accepted as the primary mechanism for regulating muscle glycogenolysis. However, additional factors than the phosphorylation state of GP have been shown to be essential in regulating the rate of glycogen degradation (Ren and Hultman 1989). Indeed, GP activity has been established to be subject to a variety of allosteric controls. The primary activator is AMP (Busby and Radda 1976), but GP activity is also enhanced by IMP, and inhibited by glucose, G-6-P, ATP and ADP (Kruszynska et al. 2001). Importantly, most of these allosteric modulators, with the exception of AMP, only have a minor influence on GPa (Morgan and Parmeggiani 1964). Therefore, while GPb activity is dependent on a relatively high AMP concentration, GPa activity is increased by low AMP concentrations but does not require it to be effective (Connett and Sahlin 1996). Generally, AMP increases GP activity by increasing its affinity for its substrate inorganic phosphate ( $\mathrm{Pi}$ ) (Kruszynska et al. 2001). However, the relative contribution of GPa and GPb to skeletal muscle glycogenolysis remains unclear.

While the role of glycogen concentration in controlling GS activity is wellestablished (Nielson et al. 2001), controversy exists regarding its importance in controlling glycogen degradation during exercise. As reviewed by Vandenberghe and co-workers (1999), most but not all studies in humans have demonstrated that initial muscle glycogen levels may cause an enhanced rate of glycogen breakdown during prolonged endurance exercise. Conversely, more often than not, muscle glycogen breakdown during short high-intensity exercise has been reported to be regulated 
irrespective of initial glycogen content (reviewed by Vandenberghe et al. 1999). High muscle glycogen concentration, in addition to increasing the availability of one of the substrate for GP, have been suggested to favour the association of GPb with the glycogen particle (see section "Glycosome - the organelle of glycogen metabolism"), hence increasing its phosphorylation (i.e. activation) by phosphorylase kinase (Madsen 1986). Furthermore, recent evidence demonstrated that during exercise, high glycogen concentrations lead to an increase in the percentage of GP in the phosphorylated form when compared to low glycogen concentrations (Shearer et al. 2001). Whether a translocation mechanism, similar to that observed for GS, exists for GP is unknown at this point.

Pi has also been proposed to play an important role in regulating GP activity (Chasiotis et al. 1982). Although its central role in controlling the glycogenolytic activity has been challenged (Ren and Hultman 1989), it is probably safe to suggest that changes in $\mathrm{Pi}$, as a substrate for $\mathrm{GP}$, are of major physiological importance for in vivo control of GP activity and that an increased in Pi concentration is essential for significant flux through the reaction to occur (Connett and Sahlin 1996).

To summarise, GP is controlled by integration of phosphorylation, allosteric modulations and substrate availability. The levels of insulin and epinephrine influence GP activity by altering its phosphorylation state. During muscle contraction, the calcium released from the sarcoplasmic reticulum acts synergistically with the rising epinephrine level to initiate glycogenolysis by activating phosphorylase kinase, which further stimulates the activation of GP by converting it to its more active "a" form (Connett and Sahlin 1996). However, the contribution of GPa and GPb to the actual glycogenolytic flux is unclear. In addition, muscle contraction initiates the hydrolysis of ATP and phosphocreatine with the release of $\mathrm{Pi}$, a substrate for GP and glycogenolysis (Ren and Hulman 1989). 


\section{Glycolysis.}

Glycolysis is the last pathway involved in glucose disposal. Glycolysis represents the sequence of reactions that converts G-6-P into pyruvate, with the concomitant production of a relatively small amount of ATP. The main regulatory steps of glycolysis between G-6-P and pyruvate are the non-equilibrium reactions catalysed by phosphofructokinase and, to a much lesser extent, pyruvate kinase (for review see Kruszynska et al. 2001). When the physiological conditions favour a low oxidative capacity (i.e. glycolytic muscle fibres), much of the pyruvate is converted to lactate and released into the circulation. On the other hand, when the physiological conditions favour a high oxidative capacity (i.e. oxidative muscle fibres), the main fate of the pyruvate is oxidative decarboxylation to acetyl CoA, which can then proceed to be metabolised by the tricarboxylic acid (TCA) cycle (Kruszynska et al. 2001). Hence, the oxidative capacity of the muscle fibre, the type and intensity of muscle contraction, as well as the physiological conditions associated with a given situation, determine the fate of pyruvate following glycolysis.

From a theoretical point of view, the glucose entering the glycolytic pathway could originate from either blood glucose uptake or glycogenolysis. Interestingly, it has recently been proposed that under specific physiological conditions in skeletal muscles (i.e. electrical stimulation of rat hindlimb muscles), glycogen serves as the main precursor of glycolysis, while glucose uptake is utilised predominantly for the synthesis of glycogen (Huang 1998). However, it has been shown that under conditions of euglycemic-hyperinsulinemic clamps, high glycogen concentrations divert blood glucose from muscle glycogen synthesis into aerobic and anaerobic glycolysis (Laurent 2000). These observations are consistent with previous in vitro rat studies that have demonstrated that muscles with high glycogen content converted less of the glucose entering the cell into glycogen and more into lactate (Young et al. 1983). These findings 
imply an additional role for muscle glycogen levels in controlling the path of glucose disposal.

\section{Glycogen turnover.}

The concept that glycogen metabolism is a dynamic process, constantly and simultaneously being synthesised and degraded, has emerged over the years. This cycling of skeletal muscle glycogen has been observed at rest (Cline et al. 1997), as well as during periods of net glycogen synthesis (Laurent et al. 2000) and breakdown (Constable et al. 1984; Huang 1998; Hutber and Bonen 1989; Nielsen et al. 2001; Price et al. 1991 and 1994). This simultaneous synthesis and degradation of glycogen has also been observed in liver (Magnusson et al. 1991). In agreement with this, numerous reports have demonstrated conditions in which, despite a constant glycogen concentration, the majority of GP remains in its active phosphorylated form (Ren and Hultman 1989 and 1990). Given the energy expensive nature of the process, the rationale behind this phenomenon remains to be established. However, the recent evidence provided by Nielsen and co-workers (2001) seems to point towards a key role for glycogen concentration in regulating this glycogen cycle. In fact, they demonstrated that while muscle contraction induced an increase in GS activity, the increase in GS activity was abolished by high skeletal muscle glycogen levels, implying that muscle contraction stimulates glycogen synthesis merely through glycogen degradation. Nielsen and colleagues (2001) also suggested that this glycogen turnover might in fact represent a "glycogen sparing" mechanism, protecting skeletal muscle against complete glycogen depletion during exercise. Further research is required to clarify the physiological purpose and mechanisms of this simultaneous glycogen synthesis and breakdown. 
To summarise, the control of each pathway involved in glycogen metabolism has been studied extensively and briefly discussed here. Enzymatic control is achieved by the integration of various mechanisms that include allosteric modulation, covalent phosphorylation, substrate and glycogen levels, protein to protein interactions, hormones, as well as the newly recognised spatial compartmentalisation accomplished by the translocation of enzymes to different subcellular locations in response to various stimuli. Based on the preceding discussion, it should be apparent that in order to provide a complete understanding of glycogen metabolism, it is essential to consider glucose transport / phosphorylation, glycolysis, glycogen synthesis and glycogenolysis as an integrated system in which no path can be analysed properly in isolation from the other pathways (Schulz 1998). Furthermore, the subcellular organisation of the enzymatic machinery and metabolic pathways must be taken into account considering the impact of this compartmentalisation on their regulation.

\section{Fibre type differences in glycogen metabolism}

It has long been recognised that skeletal muscles are composed of different types of fibres, each with different physiological and metabolic properties (for review see Hultman 1995; Pette and Staron 1990). Furthermore it is well known that skeletal muscle is not composed equally of fibres from all three types. For example, the average fibre type composition of the human vastus lateralis muscle is composed of approximately $50 \%$ type I, $40 \%$ type IIA, and $10 \%$ type IIB fibres $(3,5,34)$ Barstow et al. 1996; Bouchard et al. 1986; Simoneau et al. 1995). Hence, fibre type composition and heterogeneity of individual fibres characterise skeletal muscles. It is important to recognise the different physiological and metabolic properties of each fibre type in order to obtain a better understanding of skeletal muscle metabolism in general. In the 
present section, we will provide a brief overview of these fibre type specific differences.

Fibre type classification.

Presently, fibre type classification can be accomplished using classical histochemistry, morphometric characteristics, immuno-histochemical techniques, as well as micro-biochemical techniques (for review see Pette and Staron 1990). While the latter two techniques provide a more quantitative and precise classification under certain conditions, their usefulness in applied research is limited by the methodology involved.

On the other hand, numerous physiological experiments on skeletal muscle have reinforced the usefulness of fibre-type classification based on histochemical profiles and morphometric characteristics (Landon 1992). Morphometric parameters used to classify fibre types include Z-line width, mitochondrial shape, content and distribution, transverse tubular system appearance, and.lipid content (Landon 1992). This ultrastructuralparameter-based classification has been shown to identify, with a $90 \%$ success rate, the mammalian fast and slow twitch muscles (Eisenberg and Kuda 1976). Two different histochemical approaches allow for the separation of fibre types. One method is based upon myofibrillar actomyosin adenosine triphosphatase (ATPase) activity, and the other upon reference enzymes of anaerobic energy metabolism (Pette and Staron 1990). The combination of histochemical studies using these two methods, with a method of depleting glycogen in single motor units, made it possible to establish the relationship between metabolic properties and fatigability, and resulted in the isolation of three major fibre types in skeletal muscles (Pette and Staron 1990). These three fibre types are: (1) the slow-twitch oxidative fibres, which represent the slow fatigue-resistant motor units, commonly referred to as type $1 ;(2)$ the fast-twitch oxidative glycolytic fibres, which represent the fatigue-resistant motor units, commonly referred to as type IIA; and (3) the fast-twitch glycolytic fibres, which represent the fast-fatigable motor units, commonly 
referred to as type IIB.

These classification schemes have helped to define functional, metabolic, and molecular properties of muscle fibres selected on the basis of their histochemical appearance. Under such conditions, certain muscle fibre populations with similar functional properties and molecular profiles may be delineated. However, it should be clear that, although fibres within a particular group may contain similar structural and functional properties, the most similar muscle fibres will be those within the same motor unit. Therefore, one should keep in mind that within a muscle, a fibre type may exhibit a continuum of structural and functional properties which overlap with other fibre types (Pette and Staron 1990).

Glycogen concentration.

Findings from the limited number of experiments that have addressed the fibre type differences in glycogen content in humans generally agree in finding the type $\|$ fibres to have a greater glycogen concentration compared to type I fibres. Although some studies reported no difference in resting glycogen concentrations between fibre types in human (Essen and Henriksson 1974), the majority of studies find a 16 to $40 \%$ greater glycogen content in type II compared to type I fibres (Casey et al. 1995; Eisenberg 1983; Esbjornsson-Liljedahl et al. 1999; Essen 1978; Greenhaff et al. 1991, 1993 and 1994; Solderlund et al. 1992; Tsintzas et al. 1996; Vollestad et al. 1984 and 1989) and no difference between type IIA and IIB (Essen 1978; Vollestad et al. 1984 and 1989). In partial agreement with this, the maximal amount of glycogen that can be stored in the different fibres from rat skeletal muscles has been reported to follow the following ranked order: IIA $>$ I > IIB (Hansen et al. 2000). This information is not available in human, and since the absolute capacity for glycogen storage is significantly higher in human compared to rat skeletal muscles (Connett and Sahlin 1996; Hansen 
1999), one should be careful in extrapolating these results to human.

\section{Glycogen degradation rates.}

It is well recognised that the rate of glycogen depletion increases as exercise intensity increases (for review see Connett and Sahlin 1996). In humans, this increase in glycogenolytic rate with increasing exercise intensity is seen in all three fibre types (Vollestad et al. 1992). Furthermore, the extent of glycogenolysis in specific fibre types has also been shown to be dependent on exercise intensity (Essen 1978; Vollestad and Blom 1985). This phenomenon can be explained by the "size principle", according to which the small motor neurons innervate the type I fibres, successively larger motor neurons innervate type IIA, and the largest motor neurons innervate type IIB (Hultman 1995). The smallest neuron having the lowest threshold, a low level of exercise intensity will provide a low level of motor neuron excitation, and only some type f fibres will be recruited. With increasing excitation, more fibres are activated and with gradually increasing fractions of type IIA and with near maximal activation, type IIB will also be recruited (Hulman 1995). Accordingly, during sub-maximal light and moderate exercise, glycogen depletion is more pronounced in type I than type II fibres (Gollnick et al. 1974a and 1974b). On the other hand, at higher workloads, the type II fibres are glycogen depleted before type I fibre (Connett and Sahlin 1996) despite their greater initial glycogen content. In agreement with this, the rates of glycogen depletion during different type of maximal exercises have been demonstrated to be as much as two-fold greater in type II than type I fibres (Greenhaff et al. 1991, 1993 and 1994; Soderlund et al. 1992), with no differences between type IIA and IIB (Vollestad et al. 1992). Furthermore, this two-fold greater rate of glycogenolysis in type II compared to type I fibre is in agreement with the higher activity of GP observed in vitro in type II fibres (Harris et al. 1976). Based on these findings, the human glycogenolytic capacity of 
individual fibre types appears to be greater in type II than type I fibres.

\section{Glycogen synthesis rates.}

There is very limited information concerning the rate of glycogen synthesis in different fibre types following a glycogen depleting exercise in human. Furthermore, the results differ according to whether the analysis was performed using histochemical techniques or biochemical techniques. Piehl (1974) and Vollestad and co-workers (1989) used histochemical techniques to determine the fibre type differences in postexercise glycogen synthesis rate and both found the rate to be somewhat greater in type II compared to type I fibres. In contrast, the only two studies to have addressed this issue using biochemical techniques demonstrated the rate of glycogenesis to be faster in type I compared to type II during the first 3 hours (Casey et al. 1995) and the first 5 hours (Essen and Henriksson 1974) of recovery. Casey and colleagues (1995) explained the difference in results by the inaccuracy of the semi-quantitative nature of the histochemical techniques used. It is interesting to relate these findings to the fibre specific differences in glucose transport and glycogenin levels. Indeed, it has been shown that rat muscies composed predominantly of type I fibres have a greater plasma membrane glucose transporter content (Goodyear et al. 1991; Henrikssen et al. 1990; James et al. 1989). Furthermore, the glycogenin activity was recently demonstrated to be greater in type I compared to type II fibres (Hansen et al. 2000). Together, these findings could explain the greater glycogen synthesis rates found in type I compared to type II fibres in the early exercise recovery period.

Interestingly, Casey and colleagues (1995) actually found the rate of glycogen synthesis to be greater in type II compared to type I fibres from 3 to 10 hours postexercise. They suggested that this latter finding might be the result of the glycogenconcentration-dependent feedback inhibition of glycogen synthesis. Further studies are 
definitely required to determine the post-exercise pattern of glycogen synthesis in the different fibre types.

In conclusion, it is clear from the preceding discussion that skeletal muscles are characterised by their diversity resulting from their fibre composition and the heterogeneity of the individual muscle fibres (Pette and Staron 1990). Accordingly, any experiment that is based on enzymatic determination of a biopsy of mixed muscle fibre types will disregard the importance of this metabolic heterogeneity. Furthermore, it is apparent from the discussion of previous sections that even within a single muscle cell, there is a variety of metabolic compartments and that these may result in a heterogeneous pattern of response. In this respect, a complete understanding of glycogen metabolism must involve the acknowledgement of these fibre types differences, as well as their integration into the understanding of the different glycogen metabolising pathways.

\section{Glycosomes - the organelles of glycogen metabolism}

The concept that glycogen metabolism involves a sub-microscopic structure, comparable to other cell organelles, was first suggested in the early 1960's (Himes and Pollister 1962). A few years later, Scott and Still (1968) acknowledged that glycogen in its particulate form, is not a molecule in the ordinary static sense, but a dynamic "organelle". In their study examining the glycogen of peripheral blood leukocytes, they concluded that these organelles, which they named "glycosomes", have a discrete substructure and stable enzymatic components, held together by still undetermined structures or bonds (Scott and Still 1968). Since this acknowledgement of glycosomes, the association of glycogen with its metabolising enzymes has been accepted, and 
represents a current topic of research (for review see Roach 2001; and Rybicka 1996).

The concept of the glycogen organelle has support in the extensive biochemical literature of glycogen metabolism. The idea that glycogen is associated with protein is not novel. When Claude Bernard first isolated glycogen in the 1850's, he recognized its association with proteins, and proposed that at least some of this protein represented enzymes that degraded glycogen post-mortem (Bernard 1857). Lazarow (1942), who first extracted from the liver a high-molecular glycogen and named it particulate glycogen, found it to be associated with a very small amount of protein. However, he proposed that this protein might be of great importance in the maintenance of glycogen in the particulate state, inasmuch as all the agents that disperse the particulate glycogen markedly alter protein. Following many years of controversy regarding the artifactual nature of the protein - glycogen complex, the association of GS with the glycogen particle was established by Leloir and Goldemberg (1960). This association was confirmed by Wanson and Drochmans (1972), who completed this information by suggesting that the majority of synthase activity was found combined with the glycogen particle. Subsequently, Smythe and colleagues (1990) demonstrated that each mature glycogen particle contains an average of one GS catalytic subunit bound non-covalently. In the 1970's, the group of Fisher and co-workers extended the concept of an enzyme-glycogen complex by providing evidence to suggest that the protein-glycogen complex represents a structural and functional unit of the cell (Meyer et al. 1970). They estimated that the glycogen fraction is associated with 2 to 4 times its weight of protein. Furthermore, they established the association of muscle glycogen with GP, phosphorylase phosphatase, and phosphorylase kinase, and they confirmed that GS is also associated. However, compared to GS, GP was reported to be more readily displaced from the glycogen complex, its attachment being a function of the glycogen concentration, at least in the liver (Meyer et al. 1970). GP (and possibly GS) have been 
found to possess rather unique "glycogen storage" sites, which anchor them to the glycogen particle at sites different from their catalytic site, and facilitate their roles on the glycogen particle (Madsen 1986).

In 1972, the debranching enzyme was also identified as a component of the protein - glycogen particle from skeletal muscle (Nelson et al. 1972). A few years later, Cohen and co-workers used polyacrylamide gel electrophoresis to establish that the protein - glycogen particles isolated from skeletal muscle showed seven major proteinstaining components (Taylor et al. 1975). Of these proteins, they substantiated the presence of GS, GP, debranching enzyme, as well as the $\alpha$ and $\beta$ sub-units of phosphorylase kinase. Furthermore, Cohen (1978) concluded that GS, GP, debranching enzyme and phosphorylase kinase could account for over $95 \%$ of the protein in the protein - glycogen complex. Subsequently, the association with glycogen of protein phosphatase-1, the major active forms of protein phosphatase in skeletal muscle, was also confirmed (Cohen 1986). Finally, since the identification of the protein core of glycogen by Kennedy and colleagues (1985), it has been clearly established that glycogenin is covalently bound to the glycogen particle, and that each mature glycogen particle contains one molecule of glycogenin (Smythe and Cohen 1991). Interestingly, while all major enzymes known to be involved in glycogen metabolism have been found to be part of the glycogen - enzyme complex, only a very small proportion $(\sim 2 \%)$ of the branching enzyme has been found to be associated with the complex (Caudwell and Cohen 1980).

In line with the concept of glycosomes, recent evidence has shown the family of glycogen targeting subunits of protein phosphatase-1 to play a major role in the coordination of glycogen metabolism (for review see Newgard et al. 2000). These targeting sub-units, four of which have been identified so far, have not only been shown to target the catalytic subunit of PP1 to the glycogen particle, but also to bind 
differentially to GS, GP and phosphorylase kinase (Printen et al. 1997). These targeting proteins have been said to serve as "molecular scaffolds" by juxtaposing the enzymes with substrates in a macromolecular complex and, in the process, to exert profound effects on PP1 activity and glycogen metabolism (Newgard et al. 2000). To gain insights into the metabolic functions of protein targeting to glycogen (PTG), one of the PP1 targeting subunit expressed in various tissues including skeletal muscles, O'Doherty and co-workers (2000) recently overexpressed PTG in the liver of normal rats. Their results showed the glycogen levels to be $70 \%$ higher and the glycogenolysis in response to fasting to be decreased when compared to control. On the other hand, to evaluate the role of the muscle type of PP1 targeting subunit $\left(R_{G L}\right)$, Suzuki and colleagues (2001) recently created genetically altered (i.e. knockout) mice not expressing the $R_{\mathrm{GL}}$. Their results showed that the absence of $R_{G L}$ leads to a decrease in PP1, basal GS activities, as well as glycogen accumulation, the latter being decreased by as much as $90 \%$. While these experiments were performed with rodents and therefore, one must be careful in extrapolating these results to humans, these data provide strong evidence in favour of a predominant role of the PP1 targeting subunits in orchestrating glycogen metabolism.

In their recent experiment demonstrating the glycogen concentration dependent intracellular translocation of GS in rat skeletal muscles, Nielsen and colleagues (2001) suggested the possibility that the targeting subunits of PP1 might be involved in the GS translocation process. They proposed that one possible way in which glycogen might affect GS activity could be through interaction with the glycogen targeting subunits of PP1, which bind the catalytic subunit of PP1 to glycogen and also bind GS, GP and phosphorylase kinase. Hence, the association between glycogen levels and GS activity might be regulated by association-dissociation of proteins in the glycogen particle. In line with this hypothesis, it has been previously proposed that as the glycogen particle 
decreases in size, GS and PP1 are both released from the glycogen particle and become available for activation (Villar-Palasi 1969). Taken together, these findings indeed suggest a role for the targeting subunits of PP1 in regulating the subcellular compartmentalisation of GS. As mentioned in a previous section, a translocation mechanism for GP has not been identified.

In addition to the association of the regulatory subunits of PP1 to GS, GP, and phosphorylase kinase, other protein to protein interactions have also been demonstrated to be important in glycogen metabolism. These include the well-known interaction between GS and glycogenin (Pitcher et al. 1987), as well as the possible association of a $200 \mathrm{KDa}$ glycogenin species with phosphorylase kinase (Polishchuk et al. 1995). It is likely that many more of these protein to protein interactions will be shortly identified.

It is clear from the preceding discussion that many of the enzymes of glycogen metabolism are associated with the glycogen particle, and that enzyme to enzyme interactions are an essential component of these associations. As stated by Kruszynska and co-workers (2001), this association of substrate and product (glycogen) with synthetic and degradative enzymes substantiates the earlier proposition of the existence of glycogen as a functional organelle involved in the control of its own metabolism.

While there remains no doubt about the existence of physical associations between glycogen and the enzymes involved in its metabolism, many of the components of the glycosomes have also been demonstrated to interact with other cellular components. The association of both GS and glycogenin with the cortical actin cytoskeleton of skeletal muscles has been established by the group of Guinovart and colleagues (Banqué et al. 1997; Fernandez-Novell et al. 1997). Furthermore, the glycogen-enzyme complex has consistently been demonstrated to be physically associated with the sarcoplasmic reticulum (SR) (Goldstein et al. 1985; Rybicka 1981a and 1981b; Wanson and Drochmans 1968 and 1972; Whelan 1970). In addition, GS 
and glycogenin have also been shown to bind the SR independent of the proteinglycogen complex (Polishchuk et al. 1995). This interaction between GS and the SR has been suggested to play a role in glycogen deposition (Fernandez-Novell 1997). Recently, the $R_{G L}$ was also found to bind to the $S R$ through its strongly hydrophobic region (Tang 1991). Hubbard and Cohen (1997) suggested that the targeting of $R_{G L}$ to both glycogen and the SR might contribute to the long-recognised physical association of these structures. While the exact physiological meaning of these protein interactions remains to be clarified, the ability of the SR to sequester $\mathrm{Ca}^{2+}$ provides a potential mechanism for allosteric regulation of many of the glycogen metabolising enzymes (Kruszynska et al. 2001). In fact, a role for calcium sequestration by the SR in controlling GP has been demonstrated (Entman et al. 1980). Interestingly, the complete chain of glycolytic enzymes has also been demonstrated to differentially bind and translocate to different subcellular locations in response to different stimuli (Clarke et al. 1984; Plaxton and Storey 1986; Walsh et al. 1981), as well as to be anatomically associated with the SR membranes (Xu and Becker 1998). This could represent an efficient means of directing the product of glycogen degradation (glucosyl units) directly into the ATP producing system that is glycolysis. Furthermore, as pointed out in a previous section (see section "Glucose transport"), under specific stimuli, numerous GLUT-4 are also known to translocate to the transverse tubular system, which is physically associated with the SR. Taken together, these observations provide further evidence for the integrated scheme of control that appear to be essential for understanding glycogen metabolism.

To conclude, the existence of the glycogen particles as a structure in or by which various specific functions are carried out defines it as a cellular organelle (Rybicka 1996). Hence, the long-identified skeletal muscle glycogen $\beta$-particles (Wanson and 
Drochmans 1968) not only represents the glucose storage form of skeletal muscle glycogen, but also represents a complete enzymatic machinery with protein to protein interactions which allows it to interact with various cellular components and to control carbohydrate metabolism. As pointed out by Roach and colleagues (2001), these assemblies are likely to be dynamic, and not all interactions in the glycogen particle need to be permanent. Therefore, different assemblages may prevail at different times and in different subcellular compartments, changing with the metabolic state of the cell. Further research is required in order to provide a complete understanding of these complex interactions.

\section{Ultrastructural and morphological studies of glycosomes}

The structure of glycogen has been under investigation for nearly 150 years. As discussed in the section "Skeletal muscle glycogen structure and function", its basic internal architecture has been well established by traditional chemical and biochemical techniques. However, while these biochemical experiments have provided a substantial amount of valuable information, some essential components and characteristics of glycogen and its metabolism can only be examined by use of ultrastructural analysis. Such studies contribute to our complete understanding of the cellular and subcellular aspects of glycogen metabolism. This section will provide an overview of the work performed in the area of ultrastructural and morphological analysis of glycogen over the past 60 years.

Techniques used to enhance glycogen during TEM analysis.

Although the first histochemical method for identification of glycogen in light microscopy (periodic acid-Schiff or PAS reagent) was developed as early as the 1930's 
(Rybicka 1996), it was not until 1940 that the first electron micrographs of glycogen particles were obtained (Revel 1964). Since glycogen in its pure form diffracts the electron beam of the TEM very little, it is necessary to increase the contrast between glycogen particles and the surrounding medium (Drochmans 1963). Furthermore, it was quickly realised that the intrinsic low density of glycogen particles, in contrast to that of most cellular structures, is not appreciably increased by osmium tetroxide fixation or some electron-dense stains commonly employed in electron microscopy (Biava 1963). Owing to this lack of interaction, the preservation of glycogen for TEM was often unsatisfactory, and, even if the polysaccharide was preserved, it had such a low electron density in the tissue sections that a study of its ultrastructure was not feasible (Revel 1964).

Numerous methods have been attempted and utilised to enhance the visualisation of glycogen by transmission electron microscopy (TEM). A summary of the literature is presented in Table 1. The principal techniques used include negative staining, positive staining with potassium permanganate, standard double staining using uranyl and lead salts, treatment with reduced osmium, and histochemical staining techniques. Most of these techniques necessitate initial fixation and post-fixation of tissues in glutaraldehyde and osmium tetroxide, respectively. Negative staining involves increasing the density of the medium surrounding glycogen, thus indirectly causing the visualisation of glycogen (Drochmans 1962). The treatment with reduced osmium involves adding potassium ferrocyanide to osmium tetroxide fixation. However, the mechanism by which this treatment contrasts glycogen is not fully understood (Robinson et al. 1982). Most of the histochemical staining techniques used to enhance glycogen contrast are derived from the original technique developed by Thiery (1967). In this technique, the tissue is fixed with periodic acid-thiosemicarbazide (PA-TSH) or periodic acid- thiocarbohydrazide (PA-TCH), and subsequently stained with silver proteinate (SP) 
(Thiery 1967). The specificity of the PA-TSC-SP or PA-TCH-SP histochemical staining for polysaccharides depends on the ability of $\mathrm{HIO}_{4}$ to oxidize 1,2-glycols uniquely to form aldehydes, which subsequently react with thiosemicarbazide. The thio-group then reacts with silver to form an electron dense precipitate (Rybicka 1979). Finally, double staining with uranyl acetate and lead citrate has been used to visualise glycogen during TEM analysis. This combination is normally used for routine morphological examination of tissue sections, as it has been demonstrated to provide a good contrast for essentially all cellular components, including glycogen (Hayat 1989).

Table 1. Ultrastructural studies of glycogen.

\begin{tabular}{|c|c|c|c|c|}
\hline Authors & $\begin{array}{l}\text { Species and } \\
\text { Cell type }\end{array}$ & $\begin{array}{l}\text { Specimen } \\
\text { preparation for TEM }\end{array}$ & $\begin{array}{l}\text { Glycogen } \\
\text { characteristics }\end{array}$ & Comments \\
\hline $\begin{array}{l}\text { Drochmans } \\
1960\end{array}$ & $\begin{array}{l}\text {-Rats } \\
\text { hepatocytes }\end{array}$ & $\begin{array}{l}\text { Fixation with } 1 \% \\
\text { osmium tetroxide et } 2 \\
\% \text { potassium } \\
\text { permanganate } \\
\text {-Positive staining with } \\
5 \% \text { potassium } \\
\text { permanganate } \\
\text {-PAS treatment for } \\
\text { glycogen } \\
\text { enhancement }\end{array}$ & $\begin{array}{l}\text { - Larger particles }=60- \\
120 \mathrm{~nm} \text { diameter } \\
\text {-Smaller particles }=10-15 \\
\text { nm diameter } \\
\text {-Even smaller particles }\end{array}$ & $\begin{array}{l}\text {-Show that } \\
\text { fixation with } \\
\text { osmium } \\
\text { tetroxide } \\
\text { alone does } \\
\text { not show } \\
\text { glycogen }\end{array}$ \\
\hline $\begin{array}{l}\text { Revel et al. } \\
1960\end{array}$ & $\begin{array}{l}\text {-Frog skeletal } \\
\text { muscle, liver } \\
\text { and kidney; } \\
\text {-Fish skeletal } \\
\text { muscle } \\
\text { - Mice brown } \\
\text { adipose tissue } \\
\text {-Fractionation } \\
\text { of glycogen } \\
\text { from turtle } \\
\text { heart and } \\
\text { glycogen body } \\
\text { of the chick }\end{array}$ & $\begin{array}{l}\text {-Fixation with } 1 \% \\
\text { osmium tetroxide et } 2 \\
\% \text { potassium } \\
\text { permanganate } \\
\text {-Staining with lead } \\
\text { citrate }\end{array}$ & $\begin{array}{l}\text { - Cytoplasmic particles } \\
\text { from all tissues, roughly } \\
\text { circular in shape, range } \\
\text { from } 15-40 \mathrm{~nm} \text { diameter } \\
\text { ofrog striated muscle } \\
\text { show distinct particles } 15- \\
30 \mathrm{~nm} \text { diameter }\end{array}$ & $\begin{array}{l}\text { Original } \\
\text { criteria to } \\
\text { differentiate } \\
\text { the } \\
\text { glycogen } \\
\text { particles } \\
\text { from the } \\
\text { ribosomes }\end{array}$ \\
\hline
\end{tabular}




\begin{tabular}{|c|c|c|c|c|}
\hline $\begin{array}{l}\text { Drochmans } \\
1962\end{array}$ & $\begin{array}{l}\text {-Rat liver } \\
\text { homogenate }\end{array}$ & $\begin{array}{l}\text {-Differential } \\
\text { centrifugation } \\
\text {-Positive staining by } \\
\text { fixation with } 5 \% \\
\text { potassium } \\
\text { permanganate } \\
\text { - Negative staining } \\
\text { with potassium } \\
\text { phosphotungstate } \\
\text {-Positive and } \\
\text { negative staining are } \\
\text { compared }\end{array}$ & $\begin{array}{l}-\alpha \text { particles }=40-200 \mathrm{~nm} \\
\text { diameter (correspond to } \\
\text { the particle obtained by } \\
\text { centrifugation) } \\
-\beta \text { particles }=20-40 \mathrm{~nm} \\
\text { diameter } \\
\text { oy particles }=3 \mathrm{~nm} \\
\text { diameter }\end{array}$ & $\begin{array}{l}\text {-Original } \\
\text { article } \\
\text { describing } \\
\text { these } 3 \\
\text { particles } \\
\text { - Particles } \\
\text { dissociate } \\
\text { with } \uparrow \mathrm{pH} \text { ( } \alpha \\
\rightarrow \beta \rightarrow \gamma \text { ) }\end{array}$ \\
\hline $\begin{array}{l}\text { Biava } \\
1963\end{array}$ & $\begin{array}{l}\text {-Various } \\
\text { human tissue } \\
\text { biopsies } \\
\text { including } \\
\text { skeletal } \\
\text { muscle and } \\
\text { liver }\end{array}$ & $\begin{array}{l}\text {-Fixation with osmium } \\
\text { tetroxide } \\
\text {-Staining with lead } \\
\text { hydroxide, uranyl } \\
\text { acetate and } \\
\text { phophotungstic acid } \\
\text { (PTA) }\end{array}$ & $\begin{array}{l}\text { Liver glycogen } \rightarrow \\
\text { cytoplasmic: } \\
\text {-Glycogen rosette = 50- } \\
200 \mathrm{~nm} \text { diameter } \\
\text { associated with variable } \\
\text { amount of agranular } \\
\text { endoplasmic reticulum } \\
\text { made up of } \\
\text { - } \beta \text { particles = } 18-22 \mathrm{~nm} \\
\text { diameter } \\
\text { Liver glycogen } \rightarrow \text { intra- } \\
\text { nuclear: } \\
\text { occasional } \beta \text { particles } \\
\text { Skeletal muscle glycogen } \\
\text { - } \beta \text { particles = } 22-30 \text { nm } \\
\text { - The particles are } \\
\text { sparsely and uniformly } \\
\text { distributed within the } \\
\text { narrow sarcoplasmic } \\
\text { space between myofibrils } \\
\text {-All } \beta \text { particles reveal the } \\
\text { presence of well defined } \\
\text { round or elongated areas } \\
\text { of high density, about } 3 \\
\text { nm in diameter and } 10 \\
\text { nm in length, surrounded } \\
\text { by homogenous material } \\
\text { of lesser density }\end{array}$ & $\begin{array}{l}\text {-All } \beta \\
\text { particles } \\
\text { were } \\
\text { demonstrate } \\
\text { d to be } \\
\text { spherical in } \\
\text { shape } \\
\text { - Liver } \\
\text { glycogen } \\
\text { rosettes } \\
\text { were not } \\
\text { stained with } \\
\text { uranyl } \\
\text { acetate and } \\
\text { PTA } \\
\text { whereas } \beta \\
\text { particles } \\
\text { were }\end{array}$ \\
\hline $\begin{array}{l}\text { Mordoh } \\
\text { et al. } 1965\end{array}$ & $\begin{array}{l}\text {-Rat liver } \\
\text { homogenate }\end{array}$ & $\begin{array}{l}\text { - Centrifugation } \\
\text { - Negative staining } \\
\text { with } \\
\text { phosphotungstate }\end{array}$ & $\begin{array}{l}\text { - Larger particles = 20- } \\
180 \mathrm{~nm} \text { diameter } \\
\text { - Smaller particles }=14-25 \\
\text { nm diameter }\end{array}$ & \\
\hline $\begin{array}{l}\text { Thiery } \\
1967\end{array}$ & -Rat liver & $\begin{array}{l}\text {-Fixation with osmium } \\
\text { tetroxide and } \\
\text { glutaraldehyde with or } \\
\text { without subsequent } \\
\text { post-osmification } \\
\text {-Fixation with } \\
\text { thiocarbohydrazide } \\
\text { (T.C.H.) or thiosemi- } \\
\text { carbazide (T.S.H.) } \\
\text {-Staining with silver } \\
\text { proteinate }\end{array}$ & $\begin{array}{l}-\beta \text { particles (no diameter } \\
\text { measured) } \\
\text { - } \text { particles }=3-4 \mathrm{~nm} \\
\text { diameter }\end{array}$ & $\begin{array}{l}\text { Mostly a } \\
\text { methodolo- } \\
\text { gical paper }\end{array}$ \\
\hline
\end{tabular}




\begin{tabular}{|c|c|c|c|c|}
\hline $\begin{array}{l}\text { Wanson and } \\
\text { Drochmans } \\
1968\end{array}$ & $\begin{array}{l}\text { Rabbit } \\
\text { skeletal } \\
\text { muscie }\end{array}$ & $\begin{array}{l}\text {-Fixed in } \\
\text { glutaraldehyde } \\
\text {-Post-fixed in osmium } \\
\text { tetroxide } \\
\text {-Staining with lead } \\
\text { hydroxide }\end{array}$ & $\begin{array}{l}\text {-Dense round } \beta \text { particles, } \\
\text { regular in size and shape, } \\
\text { do not tend to form } \alpha \\
\text { cluster as in liver } \\
-\beta \text { particles }(n=500)= \\
27.3 \pm 3 \mathrm{~nm} \text { diameter } \\
\text { - } \beta \text { particles accumulate } \\
\text { free in the sarcoplasm at } \\
\text { the level of the I band, the } \\
\text { boundaries of this band } \\
\text { correspond to the T- } \\
\text { tubular system } \\
\text {-Some particles are } \\
\text { regularly aligned and } \\
\text { form bead-like strings } \\
\text { which infiltrate the } \\
\text { myofibrils in between } \\
\text { myofilaments }\end{array}$ & $\begin{array}{l}\text {-A detailed } \\
\text { analysis of } \\
\text { purified } \\
\text { glycogen } \\
\text { (free of } \\
\text { protein) is } \\
\text { also } \\
\text { performed } \\
\text { in this paper } \\
\text { (see below) }\end{array}$ \\
\hline $\begin{array}{l}\text { Wanson and } \\
\text { Drochmans } \\
1968\end{array}$ & $\begin{array}{l}\text {-Purified } \\
\text { preparation of } \\
\text { rabbit skeletal } \\
\text { muscle } \\
\text { glycogen } \beta \\
\text { particles }\end{array}$ & $\begin{array}{l}\text {-Adapted differential } \\
\text { precipitation- } \\
\text { centrifugation and } \\
\text { purification with } \\
\text { deoxycholate } \\
\text { treatment (remove } \\
\text { proteins) } \\
\text {-Fixed in } \\
\text { glutaraldehyde } \\
\text {-Post-fixed in osmium } \\
\text { tetroxide } \\
\text {-Staining with lead } \\
\text { hydroxide } \\
\text {-Negative staining } \\
\text { with } \\
\text { phosphotungstate }\end{array}$ & $\begin{array}{l}-\beta \text { particles }(n=2500)= \\
39.4 \pm 6.8 \mathrm{~nm} \text { diameter } \\
\text { - Normal distribution } \\
\text {-Possible presence of } \\
\text { subunits of about } 20 \mathrm{~nm} \\
\text { diameter }\end{array}$ & $\begin{array}{l}\text { - Direct } \\
\text { muscle } \\
\text { tissue } \\
\text { visualisation } \\
\text { is also } \\
\text { performed } \\
\text { in this paper } \\
\text { (described } \\
\text { above) } \\
\text { - Histogram } \\
\text { of diameter } \\
\text { computed }\end{array}$ \\
\hline $\begin{array}{l}\text { Scott and } \\
\text { Still } \\
1968\end{array}$ & $\begin{array}{l}\text {-Human } \\
\text { leukocytes }\end{array}$ & $\begin{array}{l}\text {-Fixation in osmium } \\
\text { tetroxide } \\
\text { - Staining with lead } \\
\text { citrate }\end{array}$ & $\begin{array}{l}\text { - Glycogen is scattered } \\
\text { randomly throughout the } \\
\text { cytoplasm in the form of } \\
\beta \text { particles ( } n=528 \text { ) }=17 \text { - } \\
25 \mathrm{~nm} \text { diameter in a } \\
\text { normal distribution } \\
\text { - } \beta \text { particles appear to be } \\
\text { composed of subunits } \\
\text { - } \beta \text { particles were } \\
\text { enlarged uniformly during } \\
\text { glycogen synthesis by the } \\
\text { addition of individual } \\
\text { glucose units (as } \\
\text { opposed to by the } \\
\text { addition of small sub- } \\
\text { particles) } \\
\text { - They suggest that the } \\
\text { number of } \beta \text { particles in } \\
\text { the cell is probably fixed } \\
\text { since degradation and } \\
\text { resynthesis involved all } \\
\text { molecules equally }\end{array}$ & $\begin{array}{l}\text { - Histogram } \\
\text { of diameter } \\
\text { computed } \\
\text { - Extraction } \\
\text { of glycogen } \\
\text { by alkali } \\
\text { produced a } \\
\text { discrete } \\
\text { particle, } \\
\text { smaller than } \\
\text { the original } \\
\beta \text { particle }\end{array}$ \\
\hline
\end{tabular}




\begin{tabular}{|c|c|c|c|c|}
\hline $\begin{array}{l}\text { Meyer et al. } \\
1970\end{array}$ & $\begin{array}{l}\text { - Rabbit } \\
\text { skeletal } \\
\text { muscle } \\
\text { derived } \\
\text { protein- } \\
\text { glycogen } \\
\text { fraction (not } \\
\text { purified } \\
\text { glycogen) }\end{array}$ & $\begin{array}{l}\text {-Comparison of } 3 \\
\text { methods to cause the } \\
\text { fragmentation } \\
\text {-Fixed in } \\
\text { glutaraldehyde } \\
\text {-Post-fixed in osmium } \\
\text { tetroxide } \\
\text {-Staining with lead } \\
\text { hydroxide }\end{array}$ & $\begin{array}{l}\text { - Glycogen particles } \\
\text { appearing as dense } \\
\text { granules }=20-30 \mathrm{~nm} \\
\text { diameter }\end{array}$ & \\
\hline $\begin{array}{l}\text { Karasaki } \\
1971\end{array}$ & $\begin{array}{l}\text { Rats } \\
\text { hepatoma } \\
\text { cells with } \\
\text { glucose } \\
\text { incubation }\end{array}$ & $\begin{array}{l}\text {-Fixed in } \\
\text { glutaraldehyde } \\
\text {-Post-fixed in osmium } \\
\text { tetroxide } \\
\text {-Double staining with } \\
\text { uranyl acetate and } \\
\text { lead citrate } \\
\text { or } \\
\text {-Treated with periodic } \\
\text { acid and stained with } \\
\text { lead }\end{array}$ & $\begin{array}{l}\text { - Cytoplasmic or intra- } \\
\text { nuclear glycogen } \\
\text { - } \alpha \text { particles ( } \uparrow \text { in size with } \\
\uparrow \text { incubation time) to up } \\
\text { to } 400 \mathrm{~nm} \\
\text { - } \beta \text { particles }=20-40 \mathrm{~nm} \\
\text { diameter } \\
\text { or particles }=4 \mathrm{~nm} \\
\text { diameter }\end{array}$ & \\
\hline $\begin{array}{l}\text { Wanson and } \\
\text { Drochmans } \\
1972\end{array}$ & $\begin{array}{l}\text {-Sarcoplasmic } \\
\text { vesicles } \\
\text { isolated from } \\
\text { rabbit skeletal } \\
\text { muscle }\end{array}$ & $\begin{array}{l}\text {-Adapted differential } \\
\text { precipitation- } \\
\text { centrifugation and } \\
\text { purification with } \\
\text { deoxycholate } \\
\text { treatment (remove } \\
\text { proteins from } \\
\text { glycogen particle) } \\
\text {-Fixed in } \\
\text { glutaraldehyde } \\
\text {-Post-fixed in osmium } \\
\text { tetroxide } \\
\text {-Double staining with } \\
\text { uranyl acetate and } \\
\text { lead citrate } \\
\end{array}$ & $\begin{array}{l}\text {-Small glycogen particles } \\
\text { were attached to the } \\
\text { outer surface of the SR } \\
\text { membrane }=10-20 \mathrm{~nm} \\
\text { diameter }\end{array}$ & $\begin{array}{l}\text {-Article } \\
\text { focused on } \\
\text { protein- } \\
\text { glycogen } \\
\text { complex } \\
\text { and not on } \\
\text { morphology } \\
\text { of glycogen } \\
\text { particles }\end{array}$ \\
\hline $\begin{array}{l}\text { Bulloch } \\
\text { et al. } 1972\end{array}$ & $\begin{array}{l}\text {-Myocardium } \\
\text { of human with } \\
\text { cardio- } \\
\text { myopathy }\end{array}$ & $\begin{array}{l}\text { - Fixed in } \\
\text { glutaraldehyde } \\
\text {-Post-fixed in osmium } \\
\text { tetroxide } \\
\text { - Double staining with } \\
\text { uranyl acetate and } \\
\text { lead citrate }\end{array}$ & $\begin{array}{l}\text { - Cytoplasmic, intra- } \\
\text { mitochondrial and intra- } \\
\text { nuclear glycogen in the } \\
\text { form of } \beta \text { particles = } 20 \text { - } \\
30 \mathrm{~nm} \text { diameter }\end{array}$ & \\
\hline $\begin{array}{l}\text { Jones and } \\
\text { Ferrans } \\
1973\end{array}$ & $\begin{array}{l}\text {-Myocardium } \\
\text { of human with } \\
\text { congenital } \\
\text { heart disease }\end{array}$ & $\begin{array}{l}\text {-Fixed in } \\
\text { glutaraldehyde } \\
\text {-Post-fixed in osmium } \\
\text { tetroxide } \\
\text { - Double staining with } \\
\text { uranyl acetate and } \\
\text { lead citrate } \\
\text { or } \\
\text {-Selected sarnples } \\
\text { treated with periodic } \\
\text { acid-T.S.H.-silver } \\
\text { proteinate }\end{array}$ & $\begin{array}{l}\text { Cytoplasmic glycogen: } \\
\text {-No } \alpha \text { particles } \\
\text { - } \beta \text { particles }=20-30 \mathrm{~nm} \\
\text { diameter } \\
\text { \% particles = } 3-5 \mathrm{~nm} \\
\text { diameter } \\
\text { (as visualised by T.S.H.) } \\
\text { Intra-mitochondrial } \\
\text { glycogen: } \\
\text { - } \text { particles }=60-250 \mathrm{~nm} \\
\text { diameter } \\
\bullet \beta \text { particles }=20-35 \mathrm{~nm} \\
\text { diameter }\end{array}$ & $\begin{array}{l}\text { - The } \beta- \\
\text { particles } \\
\text { located } \\
\text { within the } \\
\text { mitochondri } \\
\text { a were } \\
\text { slightly } \\
\text { smaller than } \\
\text { those } \\
\text { located in } \\
\text { the } \\
\text { cytoplasm }\end{array}$ \\
\hline
\end{tabular}




\begin{tabular}{|c|c|c|c|c|}
\hline & & & $\begin{array}{l}\text { \% particles = } 3-5 \mathrm{~nm} \\
\text { diameter } \\
\text { (as visualised by T.S.H.) }\end{array}$ & \\
\hline $\begin{array}{l}\text { Maron and } \\
\text { Ferrans } \\
1974\end{array}$ & $\begin{array}{l}\text {-Myocardium } \\
\text { of human with } \\
\text { cardiac } \\
\text { hypertrophy }\end{array}$ & $\begin{array}{l}\text {-Fixed in } \\
\text { glutaraldehyde } \\
\text {-Post-fixed in osmium } \\
\text { tetroxide } \\
\text { - Double staining with } \\
\text { uranyl acetate and } \\
\text { lead citrate (for } \\
\text { routine EM study) } \\
\text {-Selected samples } \\
\text { treated with periodic } \\
\text { acid-T.S.H.-silver } \\
\text { proteinate }\end{array}$ & $\begin{array}{l}\text { Froe in the cytoplasm: } \\
\text { - } \beta \text { particles }=23-35 \mathrm{~nm} \\
\text { diameter } \\
\text {-Some } \alpha \text { particles = } 130 \text { - } \\
240 \mathrm{~nm} \text { diameter made } \\
\text { up of } \beta \text { aggregates of } \beta \\
\text { particles } \\
\text { - Some intermediate } \\
\text { aggregate of 5-10 } \beta \\
\text { particles } \\
\text {-All } \beta \text { particles appeared } \\
\text { as a cluster of electron- } \\
\text { dense granules which } \\
\text { measured 3-5 nm in } \\
\text { diameter }\end{array}$ & \\
\hline $\begin{array}{l}\text { Schmalbruch } \\
\text { and } \\
\text { Kamieniecha } \\
1974\end{array}$ & $\begin{array}{l}\text { - Human } \\
\text { skeletal } \\
\text { muscle }\end{array}$ & $\begin{array}{l}\text {-Fixed in } \\
\text { glutaraldehyde } \\
\text {-Post-fixed in osmium } \\
\text { tetroxide } \\
\text { - Double staining with } \\
\text { uranyl acetate and } \\
\text { lead citrate }\end{array}$ & $\begin{array}{l}\text { - Glycogen in the } \\
\text { cytoplasm and within the } \\
\text { myofibrils in the form of } \beta \\
\text { particles }=22-30 \mathrm{~nm} \\
\text { diameter }\end{array}$ & $\begin{array}{l}\text {-Analysis of } \\
\text { subcellular } \\
\text { location and } \\
\text { fibre type } \\
\text { differences } \\
\text { were also } \\
\text { performed }\end{array}$ \\
\hline $\begin{array}{l}\text { Ferrans et al. } \\
1975\end{array}$ & $\begin{array}{l}\text { - Myocardium } \\
\text { of human with } \\
\text { cardiac } \\
\text { diseases }\end{array}$ & $\begin{array}{l}\text {-Fixed in } \\
\text { glutaraldehyde } \\
\text {-Post-fixed in osmium } \\
\text { tetroxide } \\
\text { - Double staining with } \\
\text { uranyl acetate and } \\
\text { lead citrate } \\
\text {-Selected samples } \\
\text { treated with periodic } \\
\text { acid-T.S.H.-silver } \\
\text { proteinate }\end{array}$ & $\begin{array}{l}\text { Intra-nuclear glycogen } \\
\text { (7 \% of patients): } \\
-\beta \text { particles = } 16-36 \mathrm{~nm} \\
\text { diameter } \\
\text { - } \beta \text { particles were either } \\
\text { isolated and randomly } \\
\text { dispersed or as small } \\
\text { aggregates } \\
\text { Cytoplasmic glycogen: } \\
\text { - Few glycogen rosettes in } \\
\text { cytoplasm of cells which } \\
\text { did not have intra-nuclear } \\
\text { glycogen } \\
-\beta \text { particles = } 16-36 \mathrm{~nm} \\
\text { diameter } \\
-\beta \text { particles were either } \\
\text { dispersed throughout or } \\
\text { arranged in short strands }\end{array}$ & \\
\hline $\begin{array}{l}\text { Rybicka } \\
1979\end{array}$ & $\begin{array}{l}\text {-Dog cardiac } \\
\text { conductive } \\
\text { fibres }\end{array}$ & $\begin{array}{l}\text { - Fixed in } 3 \% \\
\text { glutaraldehyde } \\
\text {-Post-fixed in } 1 \% \\
\text { osmium tetroxide } \\
\text { - Some sections were } \\
\text { histochemically } \\
\text { stained with periodic } \\
\text { acid- } \\
\text { thiosemicarbazide- } \\
\text { silver proteinate } \\
\text {-Other sections were } \\
\text { stained with uranyl } \\
\text { acetate and lead } \\
\text { citrate }\end{array}$ & $\begin{array}{l}\text { PA-TSC-SP staining: } \\
\text {-Cell structure were un- } \\
\text { contrasted except for } \\
\text { glycogen, which } \\
\text { appeared as } ~ 3 \mathrm{~nm} \\
\text { particles arranged in } \\
\text { aggregates } 20-30 \mathrm{~nm} \text { in } \\
\text { diameter } \\
\text { Uranyl acetate and lead } \\
\text { citrate staining: } \\
\text {-Well contrasted cellular } \\
\text { structures and } \beta \text { particles } \\
=20-30 \mathrm{~nm} \text { diameter }\end{array}$ & $\begin{array}{l}\text { - Conclusion } \\
\text { that the 20- } \\
30 \text { nm } \\
\text { granules } \\
\text { visualised } \\
\text { by U / Pb } \\
\text { represent } \\
\text { proteins } \\
\text { bound to } \\
\text { glycogen to } \\
\text { form cellular } \\
\text { organelles } \\
\text { called } \\
\text { glycosomes }\end{array}$ \\
\hline
\end{tabular}




\begin{tabular}{|c|c|c|c|c|}
\hline $\begin{array}{l}\text { Rybicka } \\
\text { 1981a }\end{array}$ & $\begin{array}{l}\text { - Dog cardiac } \\
\text { conductive } \\
\text { fibres }\end{array}$ & $\begin{array}{l}\text {-Fixed in } 3 \% \\
\text { glutaraldehyde } \\
\text {-Post-fixed in } 1 \% \\
\text { osmium tetroxide } \\
\text {-Some blocks were } \\
\text { dehydrated directly } \\
\text { after fixation, while } \\
\text { others were treated } \\
\text { with uranyl acetate } \\
\text { before dehydration } \\
\text { - Histochemical } \\
\text { staining was } \\
\text { performed with } \\
\text { periodic acid- } \\
\text { thiosemicarbazide- } \\
\text { silver proteinate } \\
\text {-In control sections, } \\
\text { the periodic acid was } \\
\text { replaced by } \mathrm{H}_{2} \mathrm{O}_{2} \\
\text {-Both histochemical } \\
\text { and control section } \\
\text { were post-stained } \\
\text { with uranyl acetate } \\
\text { and lead citrate }\end{array}$ & $\begin{array}{l}\text { Double staining } \\
\text { tochnique: } \\
\text {-lrregularly spheroid } \\
\text { shaped } \beta \text { particles = 20- } \\
30 \text { nm diameter } \\
\text {-y particles = - } 3 \text { nm } \\
\text { diameter } \\
\text { Control section: } \\
\text { - Regular spheroid } \beta \\
\text { particles = 20-30 nm } \\
\text { diameter } \\
\text { Technical issues: } \\
\text {-Protein was poorly } \\
\text { contrasted is sections } \\
\text { stained histochemically } \\
\text { without heavy metals } \\
\text {-Glycogen appeared in all } \\
\text { sections stained by PA- } \\
\text { TSC-SP } \\
\text {-Fixation with uranyl } \\
\text { acetate before } \\
\text { dehydration caused } \\
\text { disappearance of } \\
\text { glycosomes not } \\
\text { associated with cellular } \\
\text { components but not that } \\
\text { associated with cellular } \\
\text { component (SR, } \\
\text { intermediate filaments) } \\
\rightarrow \text { because the acidity of } \\
\text { the fixation dissociate the } \\
\text { CHO and protein } \\
\text { component of the } \\
\text { glycosome } \\
\text { - This indicates the } \\
\text { different degrees of } \\
\text { resistance to acidic } \\
\text { treatment of free and } \\
\text { bound glycosomes, and } \\
\text { suggests that glycosomes } \\
\text { form stable associations } \\
\text { with other cellular } \\
\text { structures }\end{array}$ & $\begin{array}{l}\text {-This paper } \\
\text { demonstra- } \\
\text { tes a } \\
\text { technique to } \\
\text { stain } \\
\text { simultaneou } \\
\text { sly and } \\
\text { specifically } \\
\text { both the } \\
\text { protein and } \\
\text { CHO } \\
\text { components } \\
\text { of } \\
\text { glycosomes } \\
\text {-Conclusion } \\
\text { that the } \\
\text { periodic } \\
\text { acid (HIO }) \\
\text { component } \\
\text { of PA-TSC- } \\
\text { SP } \\
\text { interferes } \\
\text { with the } \\
\text { reaction of } \\
\text { U / Pd } \\
\text { stains with } \\
\text { cellular } \\
\text { proteins. } \\
\text { also due to } \\
\text { it's acidic } \\
\text { component } \\
\text { which } \\
\text { denature } \\
\text { proteins }\end{array}$ \\
\hline $\begin{array}{l}\text { Rybicka } \\
\text { 1981b }\end{array}$ & $\begin{array}{l}\text {-Dog cardiac } \\
\text { conductive } \\
\text { fibres }\end{array}$ & $\begin{array}{l}\text {-Fixed in } 3 \% \\
\text { glutaraidehyde } \\
\text {-Post-fixed in } 1 \% \\
\text { osmium tetroxide } \\
\text {-Some blocks were } \\
\text { dehydrated directly } \\
\text { after fixation, while } \\
\text { others were treated } \\
\text { with uranyl acetate } \\
\text { before dehydration } \\
\text {-Some sections were } \\
\text { histochemically } \\
\text { stained with periodic } \\
\text { acid- } \\
\text { thiosemicarbazide- }\end{array}$ & $\begin{array}{l}\text {-Glycosomes were found } \\
\text { to be attached to } \\
\text { membranes of the } \\
\text { endoplasmic reticulum } \\
\text { and to intermediate } \\
\text { filaments } \\
\text { - The size and number of } \\
\text { glycogen particles were } \\
\text { fairly uniform in samples } \\
\text { taken from the same dog. } \\
\text { but differed among } \\
\text { specimens } \\
\rightarrow \text { samples from some } \\
\text { dogs contained large } 25 \\
\text { nm diameter glycosomes. }\end{array}$ & $\begin{array}{l}\text {-The size of } \\
\text { glycosomes } \\
\text { was } \\
\text { correlated } \\
\text { with the } \\
\text { density of } \\
\text { SR and } \\
\text { filaments } \\
\text {-The } \\
\text { present } \\
\text { demonstrati } \\
\text { on that } \\
\text { glycosomes } \\
\text { are attached } \\
\text { to SR in the }\end{array}$ \\
\hline
\end{tabular}




\begin{tabular}{|c|c|c|c|c|}
\hline & & $\begin{array}{l}\text { silver proteinate (for } \\
\text { CHO) and with uranyl } \\
\text { acetate and lead } \\
\text { citrate (for proteins) } \\
\text {-Other control } \\
\text { sections were post- } \\
\text { stained with uranyl } \\
\text { acetate and lead } \\
\text { citrate alone }\end{array}$ & $\begin{array}{l}\text { which appeared as } \\
\text { regular spheroid } \\
\text { organelles rich in } \\
\text { particles of glycogen } \\
\rightarrow \text { glycosomes in other } \\
\text { specimens were small } \\
\text { (17 nm diameter) and } \\
\text { irregularly shaped } \\
\text { - Both small and large } \\
\text { glycosomes were } \\
\text { attached to other cellular } \\
\text { structures, which suggest } \\
\text { that the binding was } \\
\text { independent of the total } \\
\text { level of glycogen in the } \\
\text { organelle } \\
\text {-Evidence to suggest that } \\
\text { the formation of a bond } \\
\text { between glycosomes and } \\
\text { ER or filaments is } \\
\text { associated with changes } \\
\text { in chemical or physical } \\
\text { structure of glycosomes }\end{array}$ & $\begin{array}{l}\text { intact cells } \\
\text { provides the } \\
\text { first } \\
\text { histochemic } \\
\text { al and } \\
\text { morphologic } \\
\text { al support } \\
\text { for the } \\
\text { biochemical } \\
\text { findings }\end{array}$ \\
\hline $\begin{array}{l}\text { Sjostrom } \\
\text { et al. } 1982\end{array}$ & $\begin{array}{l}\text {-Human } \\
\text { skeletal } \\
\text { muscles }\end{array}$ & $\begin{array}{l}\text {-Fixed in } 2.5 \% \\
\text { glutaraldehyde } \\
\text {-Post-fixed in } 1 \% \\
\text { osmium tetroxide } \\
\text { - Treated with periodic } \\
\text { acid- } \\
\text { thiosemicarbazide- } \\
\text { silver proteinate }\end{array}$ & $\begin{array}{l}\text { - Subsarcolemmal, inter- } \\
\text { myofibrillar and intra- } \\
\text { myofibrillar } \beta \text {-particles } \\
\text { (diameter not reported) }\end{array}$ & $\begin{array}{l}\text {-Exercise } \\
\text { depletion } \\
\text { and fibre } \\
\text { type } \\
\text { differences } \\
\text { were } \\
\text { performed }\end{array}$ \\
\hline $\begin{array}{l}\text { Devos et al. } \\
1983\end{array}$ & $\begin{array}{l}\text {-Rat } \\
\text { hepatocytes }\end{array}$ & $\begin{array}{l}\text { - Fixation with osmium } \\
\text { tetroxide and } \\
\text { potassium } \\
\text { ferricyanide } \\
\text { - Double staining with } \\
\text { uranyl acetate and } \\
\text { lead citrate }\end{array}$ & $\begin{array}{l}\text { - } \alpha \text { particles }=35-140 \mathrm{~nm} \\
\text { diameter } \\
\text {-Both the number and } \\
\text { size of the glycogen } \\
\text { particles were affected by } \\
\text { glycogen concentration } \\
\text { where a } 7 \text {-fold } \uparrow \text { in } \\
\text { glycogen concentration }= \\
1.8 \text {-fold } \uparrow \text { in particle } \\
\text { volume and a } 4 \text { fold } \uparrow \text { in } \\
\text { number of glycogen } \\
\text { particles } \\
\text { The average } M_{r} \text { of } \\
\text { glycogen particle as } \\
\text { measured from average } \\
\text { particle size varied from } \\
89 \times 10^{6} \text { to } 161 \times 10^{6} \\
\end{array}$ & $\begin{array}{l}\text { Only the } \alpha \\
\text { particles } \\
\text { were } \\
\text { analysed. }\end{array}$ \\
\hline $\begin{array}{l}\text { Goldstein } \\
\text { et al. } 1985\end{array}$ & $\begin{array}{l}\text {-Dog cardiac } \\
\text { muscle tissue } \\
\text { and } \\
\text {-SR isolated } \\
\text { fraction from } \\
\text { dog } \\
\text { ventricular } \\
\text { cardiac } \\
\text { muscle } \\
\end{array}$ & $\begin{array}{l}\text { - Density gradient } \\
\text { centrifugation } \\
\text {-Fixed in } \\
\text { glutaraldehyde } \\
\text {-Post-fixed in } 1 \% \\
\text { osmium tetroxide } \\
\text { - Double staining with } \\
\text { uranyl acetate and } \\
\text { lead citrate } \\
\end{array}$ & $\begin{array}{l}\text { For both Intact control } \\
\text { muscle tissue and } \\
\text { Isolated SR fraction: } \\
\text { - } \beta \text { particles }=20-30 \mathrm{~nm} \\
\text { diameter } \\
\text { - Characterised by } \\
\text { roughly spherical } \\
\text { electron-dense } \\
\text { aggregations }\end{array}$ & $\begin{array}{l}\text {-They } \\
\text { provide } \\
\text { support for } \\
\text { the } \\
\text { biochemical } \\
\text { finding of a } \\
\text { structured } \\
\text { enzyme } \\
\text { complex } \\
\end{array}$ \\
\hline
\end{tabular}




\begin{tabular}{|c|c|c|c|c|}
\hline & & & $\begin{array}{l}\text {-Some of the particles } \\
\text { adhered closely to the SR } \\
\text { membrane, and these } \\
\text { were more resistant to } \\
\text { breakdown by gluco- and } \\
\alpha \text {-amylase } \\
\text { Intact control muscle } \\
\text { tissue only: } \\
\text {-Particles detected } \\
\text { between the myofibrils } \\
\text { and beneath the } \\
\text { sarcolemma } \\
\text { Isolated SR fraction only } \\
\text {-Particles occurred } \\
\text { singly, in clusters, or } \\
\text { strung together by a fine } \\
\text { thread }\end{array}$ & $\begin{array}{l}\text { involving the } \\
\text { SR and } \\
\text { glycogeno- } \\
\text { Iytic } \\
\text { enzymes }\end{array}$ \\
\hline $\begin{array}{l}\text { Friden et al. } \\
1985\end{array}$ & $\begin{array}{l}\text {-Human } \\
\text { skeletal } \\
\text { muscles }\end{array}$ & $\begin{array}{l}\text {-Fixed in } 2.5 \% \\
\text { glutaraldehyde } \\
\text {-Post-fixed in } 1 \% \\
\text { osmium tetroxide } \\
\text { - Treated with periodic } \\
\text { acid- } \\
\text { thiosemicarbazide- } \\
\text { silver proteinate }\end{array}$ & $\begin{array}{l}\text { - Subsarcolemmal, inter- } \\
\text { myofibrillar and intra- } \\
\text { myofibrillar } \beta \text {-particles = } \\
25 \mathrm{~nm} \text { diameter }\end{array}$ & $\begin{array}{l}\text {-Exercise } \\
\text { depletion } \\
\text { and fibre } \\
\text { type } \\
\text { differences } \\
\text { were } \\
\text { performed }\end{array}$ \\
\hline $\begin{array}{l}\text { Friden et al. } \\
1989\end{array}$ & $\begin{array}{l}\text { Human } \\
\text { skeletal } \\
\text { muscles }\end{array}$ & $\begin{array}{l}\text {-Fixed in } 2.5 \% \\
\text { glutaraldehyde } \\
\text {-Post-fixed in } 1 \% \\
\text { osmium tetroxide } \\
\text { - Treated with periodic } \\
\text { acid- } \\
\text { thiosemicarbazide- } \\
\text { silver proteinate }\end{array}$ & $\begin{array}{l}\text { Two separate populations } \\
\text { of } \beta \text { particles were } \\
\text { distinguished in the } \\
\text { myofibrillar locations: } \\
\text { - Heavily stained } 18-30 \\
\text { nm diameter }(n=110) \\
\text { - Lighter particles } 45-60 \\
\text { nm diameter ( } n=35)\end{array}$ & $\begin{array}{l}\text {-Exercise } \\
\text { depletion } \\
\text { and fibre } \\
\text { type } \\
\text { differences } \\
\text { were } \\
\text { performed } \\
\text {-Histogram } \\
\text { of diameter } \\
\text { computed }\end{array}$ \\
\hline
\end{tabular}

Morphology of glycogen.

The first comprehensive series of experiments using TEM analysis to visualise glycogen was performed by Drochmans in the early 1960s (Drochmans 1960, 1962 , 1963). Drochmans (1960) established the identity of glycogen as stainable material by isolating chemically identifiable fractions of glycogen and by establishing that such fractions had the same morphology and staining characteristics as those of intracellular glycogen. He used liver fractions consisting of pure glycogen and confirmed that the appearance of glycogen in these pellets showed large particles identical in appearance 
to those described previously in mouse liver (Luft 1956). A few years later, Drochman (1962) studied negatively-stained glycogen isolated from liver, and showed that liver glycogen was organised in an ordered arrangement of particles of different size, the $\alpha, \beta$ and $\gamma$ particles of glycogen. The $\alpha$ particles were the largest species of glycogen, $40-$ $200 \mathrm{~nm}$ in diameter. The $\alpha$ particle comprised smaller $20-30 \mathrm{~nm}$ spheroid $\beta$ particles. The $\gamma$ particles were identified as $-3 \mathrm{~nm}$ subunits of the $\beta$ particles. This three-level hierarchically ordered subunit arrangement of glycogen was subsequently observed under different experimental conditions including the use of lead stained tissue fixed with potassium permanganate (Revel 1964), the use of triple staining with lead, uranyl acetate and phosphostungstic acid (Biava 1963), and the use of histochemical glycogen staining (Jones and Ferrans 1973; Karasaki 1971; Maron and Ferrans 1974; Rybicka 1979, 1981a and 1981b; Thiery 1967).

A few years later, Wanson and Drochmans (1968) performed a detailed analysis of skeletal muscle glycogen in rabbit using lead hydroxide staining. They found that the glycogen in skeletal muscle was made of dense and round $\beta$ particles, regular in size and shape, and which did not tend to aggregate to form $\alpha$ particles as in liver. They further manually computed the diameter of $\mathbf{5 0 0}$ particles and found an average diameter of $27.3 \pm 3 \mathrm{~nm}$. No mention of $\gamma$ particle subunits was made. Interestingly, the organisation of $\beta$ particles as aggregates of $\gamma$ particle subunits was not observed in any subsequent studies that used heavy metal staining alone (Bulloch et al. 1972; Meyer et al. 1970; Wanson and Drochmans 1968 and 1972). Although both the $\alpha$ and $\beta$ particles were observed in liver examined by this technique, the $\beta$ particles in both muscle and liver appeared simply as regular spheroid densely stained particles, without $\gamma$ subunits. Since the most common TEM procedures used sections stained with uranyl acetate (U) and lead citrate $(\mathrm{Pb})$, and this staining showed structures corresponding to the $\alpha$ and $\beta$ 
particles initially described by Drochmans (1962), the U / Pb-stained structures became generally accepted as units of native glycogen in tissues (Rybicka 1996).

These TEM observations were subsequently clarified by the demonstration that the different staining techniques described above in fact reacted with dissimilar components in the structure accepted earlier as simple glycogen particles (for review see Rybicka 1996). Based on the earlier biochemical evidence that the glycogen particles are associated with the enzymes of its metabolism (see section "Glycosomes the organelies of glycogen metabolism"), Rybicka provided the first morphological identification of the protein and glycogen components of glycosomes by TEM (Rybicka 1979). Furthermore, she confirmed that the protein and glycogen components are normally bound to each other, forming the structure with a 20-30 nm diameter (Rybicka 1979). In a subsequent experiment in cardiac muscle, Rybicka (1981a) succeeded to clarify the different components stained with the different staining techniques. She demonstrated that control samples stained with U / Pd showed 20-30 $\mathrm{nm}$ spheroid granules and that histochemically-stained sections (PA-TSH-SP), showed glycogen as 3 $\mathrm{nm}$ particles aggregated into $20-30 \mathrm{~nm} \beta$ particles (Rybicka 1981a). She provided evidence to suggest that the $\mathrm{CHO}$ moiety of the glycosome was not stained with heavy metals ( $U / P d)$, and that the $U / P d$ stained particle in fact represent the protein moiety of the glycosome. Hence, the staining of glycogen by heavy metals (U / Pd) is in fact due to the reaction of glycosomal protein, as is the reaction between cyanides and glycosomal proteins. The reactions of proteins with cyanides and ferrocyanides are wellestablished histochemical indicators of sulphur-containing amino acids (Rybicka 1996). Together, these results lead to the conclusion that the structures that are generally considered as glycogen particles in TEM, in fact represent glycosomes, organelles composed of protein (stainable with $\mathrm{U} / \mathrm{Pd}$ or ferrocyanide) and $\mathrm{CHO}$ (stainable 
histochemically) (Rybicka 1996). However, as pointed out by Rybicka (1996), neither the recognition of glycosomes as cellular organelles, nor the microscopic identification of their components have been widely appreciated.

\section{Size of glycogen particles.}

An extensive analysis of the structural features of particulate glycogen in various human cell types was made from biopsy specimens using triple staining technique (Biava 1963). In addition to its presence in liver and skeletal muscle cells, glycogen, in the form of $\beta$ particles, was identified in variable amounts in numerous cells. These include cells of the nephron; parathyroid chief cell; platelets; neutrophils; practically all endothelial cells including smooth muscle cells of arterioles and venules, muscular arteries and veins; peritoneal mesothelial cells; and nerve axons and Schwann's cells of peripheral un-myelinated nerves. From his comprehensive survey, Biava (1963) confirmed and extended the previous findings of Drochmans (1962) and concluded that the average size of the $\beta$ particles in different cells varied between 18 and $30 \mathrm{~nm}$, but remained fairly constant within cells of the same type. Furthermore, he stated that in any cell type, the amount of glycogen is highly variable from one cell to the next. Finally, he concluded that with regards to the structural forms of glycogen, in humans as in various animal species, aggregates of particles characterising glycogen rosettes ( $\alpha$ particles) are normally restricted to the cytoplasm of liver cells, whereas isolated $\beta$ particles are prevalent in all other locations.

In line with these findings, Revel (1964) extensively reviewed the literature on the electron microscopy of glycogen, and concluded that the glycogen $\beta$ particles appear roughly spherical in shape, and are 15 to $40 \mathrm{~nm}$ in diameter, depending on the cell type. Subsequently, only a few laboratories have examined the size of glycogen particles in 
various intact tissues. A summary of the literature is presented in Table 1 . While most of these studies solely provided gross estimates of $\beta$ particles diameter (14 to $60 \mathrm{~nm}$ ), very few actually measured $\beta$ particle diameter (Friden et al. 1989; Scott and Still 1968; Wanson and Drochmans 1968). Wanson and Drochmans (1968) examined rabbit skeletal muscles using staining with lead hydroxide. They measured the diameter of 500 particles and found a mean diameter of $27.3 \pm 3 \mathrm{~nm}$. Interestingly, they also measured the diameter of 2500 purified glycogen particles obtained from the rabbit skeletal muscle preparation, and found the mean diameter of $\beta$ particles to be $39.4 \pm 6.8 \mathrm{~nm}$. They further stated that the particle diameters were normally distributed, and suggested the possible presence of sub-units of about $20 \mathrm{~nm}$ in diameter (in the purified glycogen fraction). Scott and Still (1968), on the other hand, studied human leukocytes using staining with lead citrate. They found the diameter of $528 \beta$ particles to be normally distributed between 17 and $25 \mathrm{~nm}$. Finally, Friden and colleagues (1989) examined human skeletal muscles using the PA-TCH-SP technique, and found a bimodal distribution of $\beta$ particles. The smaller particles were heavily stained and $18-30 \mathrm{~nm}$ in diameter $(n=110)$, while the larger ones appeared as lighter and $45-60 \mathrm{~nm}$ in diameter $(n=35)$.

Therefore, while the majority of the experiments found $\beta$ particle glycogen to range between 15 and $40 \mathrm{~nm}$ in diameter, two studies found a diameter appreciably larger ranging up to $60 \mathrm{~nm}$ in diameter in both purified muscie glycogen (Wanson and Drochmans 1968) and intact skeletal muscles (Friden et al. 1989). To explain the finding by Wanson and Drochmans (1968), Meyer and co-workers (1970) suggested that the difference could be caused by the aggregation of purified glycogen particles. This finding was later confirmed by Rybicka (1981a), who demonstrated that glycogen is normally secured in intact glycosomes due to the fixation of the associated protein. After 
removal of the proteins, as in glycogen purification (Wanson and Drochmans 1968), the unfixed glycogen from disrupted glycosomes floats in the cell and aggregates into large clumps. These evidences also explain the greater particle size obtained by Friden and colleagues (1989). In their study, the periodic acid in the PA-TSC-SP procedure after routine fixation caused the dissociation of the protein from the $\mathrm{CHO}$ moiety, thus causing the aggregation of the protein free glycogen as bigger particles. For reasons that are still unexplained, part of the glycogen that is associated with the cellular protein components, including the SR or the myofibrils, are relatively "protected" from this acidrelated dissociation, and conserve their normal $\beta$ particle diameter (Rybicka 1996). It is interesting to relate this information to the morphology of liver glycogen as an $\alpha$ particle or rosette. Liver glycogen has been reported to be composed of a very heterogeneous population, ranging in molecular weight to $>10^{\circ} \mathrm{Da}$ (Calder and Geddes 1985). This is in contrast to the maximal skeletal muscle $\beta$ particle $M_{r}$ of $10^{7} \mathrm{Da}$ (Wanson and Drochmans 1968). The aggregation of $\beta$ particles in the liver could be linked to its lesser protein content relative to skeletal muscle glycogen (Smythe et al. 1989). Objective evidence is required to confirm this hypothesis.

From the foregoing data, it appears that glycogen from intact tissues of human and different species is structurally defined by spherical particles of variable size $(\sim 14$ to $40 \mathrm{~nm}$ ), composed of a different number of unit filaments of approximately $3 \mathrm{~nm}$ in diameter. These particles exist either in an aggregated or isolated form, respectively responsible for the differing appearances of glycogen as $\alpha$ particles in liver and as $\beta$ particles in extra-hepatic tissues (including mostly striated muscles). 
It is interesting to relate these findings to the range of $\beta$ particle diameter estimated by mathematical modelling (see section "Skeletal muscle glycogen structure and function $\left.{ }^{n}\right)$. According to Goldsmith and colleagues (1982), the mature $\beta$ particle in skeletal muscles has a maximal diameter of $42 \mathrm{~nm}$. This value is in close agreement with the range of $\beta$ particle diameter as obtained by direct visualisation using TEM analysis.

As discussed in the section "Glucose disposal and glycogen metabolism in skeletal muscles", the concept that pro-glycogen is a stable intermediate in the biosynthesis of glycogen has been proposed (Alonso et al. 1995). While this concept has been challenged based on biochemical evidence (Roach and Skurat 1997), the available TEM-based evidence of a normal distribution of $\beta$ particles (Scott and Still 1968; Wanson and Drochmans 1968) provides further grounds against the concept.

Subcellular location of glycogen in skeletal muscles.

A limited number of investigations have examined the subcellular distribution of glycogen in skeletal muscles. Table 2 summarises the available literature. The area was first addressed by Schmalbruch and Kamieniecha (1974), who examined skeletal muscle biopsies from 5 human subjects. They identified the majority of $\beta$ particles as loosely arranged or packed in strands in the cytoplasm, and marking boundaries of the myofibrils in the I band region. They also identified some particles within the myofibrils, either as rows between myofilaments of the $A$ band, or as single granules between myofilaments of the I band. Although the observations reported in that study were novel, they were very qualitative in nature. 
The issue was subsequently addressed by the laboratory of Ekblom and coworkers (Friden et al. 1985 and 1989; Sjostrom et al. 1982) in the 1980s. The results of the first two of these studies (Friden et al. 1985; Sjostrom et al. 1982) extended the results of Schmalbruch and Kamieniecha (1974) to show that resting human skeletal muscle glycogen is located in three distinct subcellular locations: the subsarcolemmal space, the inter-myofibrillar space, and the intra-myofibrillar space. They characterised the subsarcolemmal glycogen as particles found in large accumulations. The intermyofibrillar space was defined as glycogen particles found between myofibrils, especially at the level of the I-band were component of the SR and mitochondria are also located. Finally, the intra-myofibrillar glycogen was described as rows of particles seen as if they occupied a space previously filled with thin or thick myofilaments. The intra-myofibrillar glycogen was hence the particles found between myofilaments, especially in the I band and some in the A band.

The third and last study performed by this laboratory (Friden et al. 1989) complemented the studies discussed above and further characterised each of the subcellular locations. The subsarcolemmal space was characterised as large focal clusters of glycogen particles found around the mitochondria within this space. The clusters occurred irregularly but always in proximity to the mitochondria. As found previously, the inter-myofibrillar space glycogen accumulation were located mostly at the level of the I band, although these clusters frequently extended over the length of several sarcomeres. Friden and co-workers (1989) also suggested that quantitatively, the inter-myofibrillar glycogen appears to be the most important glycogen compartment. They suggested that it is not surprising to find the most important intra- muscular glycogen depot in the inter-myofibrillar region in close proximity with the SR at the I band level, given the well known association of the glycosomes and glycolytic enzymes with the SR (Friden et al. 1989). In fact, the physical association of the glycosomes with the 
SR has been demonstrated consistently as previously discussed (see section on

"Glycosomes - the organelle of glycogen metabolism"). Finally, they proceeded to a

detailed analysis of the intra-myofibrillar glycogen and divided it into three sub-

compartments. These intra-myofibrillar sub-compartments of glycogen were the Para-Z-

disc, the $\mathrm{N}_{2}$-line, and the $\mathrm{H}$-zone. Details of each are provided in Table 2.

Table 2. Subcellular location of the glycogen particles in human skeletal muscles.

\begin{tabular}{|c|c|c|c|}
\hline Author & $\begin{array}{l}\text { Subjects I } \\
\text { protocol }\end{array}$ & $\begin{array}{l}\beta \text { particle } \\
\text { size }\end{array}$ & ion \\
\hline $\begin{array}{l}\text { Schmalbruch } \\
\text { and } \\
\text { Kamieniecha } \\
1974\end{array}$ & $\begin{array}{l}\text {-Biopsies } \\
\text { from the } \\
\text { Biceps } \\
\text { brachial of } 5 \\
\text { normal } \\
\text { subjects } \\
.21-65 \text { y.o. }\end{array}$ & $\begin{array}{l}-22-33 \mathrm{~nm} \\
\text { diameter }\end{array}$ & $\begin{array}{l}\text { - Inter-myofibrillar: loosely arranged or packed in } \\
\text { strands in the cytoplasm marking boundaries of } \\
\text { the myofibrils in the I band region } \\
\text { - Intra-myofibrillar: formed rows between } \\
\text { myofilaments of the A band } \\
\text { ointra-myofibrillar: single granules between } \\
\text { myofilaments of the I band }\end{array}$ \\
\hline $\begin{array}{l}\text { Sjostrom ot } \\
\text { al. } 1982\end{array}$ & $\begin{array}{l}\text {-Biopsies } \\
\text { from the } \\
\text { vastus } \\
\text { lateralis of } 10 \\
\text { healthy men } \\
\text {-25-35 y.0. } \\
\text {-Groups: } \\
1 . \text { Resting } \\
\text { control }(n=6) \\
2.30 \mathrm{~km} \text { run } \\
(n=4) \\
\bullet \text { Biopsy was } \\
\text { obtained } \\
\text { approximately } \\
40 \text { minutes } \\
\text { post-exercise }\end{array}$ & $\begin{array}{l}\text { - Inter- } \\
\text { myofibrillar } \\
\text { glycogen } \\
\text { particles } \\
\text { remaining } \\
\text { post-exercise } \\
\text { were smaller } \\
\text { compared to } \\
\text { control } \\
\text {-Intra- } \\
\text { myofibrillar } \\
\text { glycogen } \\
\text { particles } \\
\text { remaining } \\
\text { post-exercise } \\
\text { was of a } \\
\text { normal size }\end{array}$ & $\begin{array}{l}\text { Resting control: } \\
\text {-Subsarcolemmal: glycogen particles found in } \\
\text { large subsarcolemmal accumulation } \\
\text {-Inter-myofibrillar: glycogen particles found } \\
\text { between myofibrils, especially at the level of the } \\
\text { 1-band were component of the SR and } \\
\text { mitochondria are also located } \\
\text { ointra-myofibrillar: glycogen particles found } \\
\text { between myofilaments, especially in the I band, } \\
\text { and some in the A band. Also, rows of particles } \\
\text { were seen as if they occupied a space previously } \\
\text { filled with thin or thick myofilaments } \\
\text { Exercised individual: depleted fibres } \\
\text { - No subsarcolemmal accumulations } \\
\text {-Practically no inter-myofibrillar glycogen } \\
\text { particles were found and the remaining were of a } \\
\text { smaller sizes } \\
\text {-Shorter rows of normal size particles located in } \\
\text { the I-band were relatively frequent (intra- } \\
\text { myofibrillar) } \\
\text { - The particles present post-exercise were mainly } \\
\text { intra-myofibrillar }\end{array}$ \\
\hline \begin{tabular}{|l} 
Friden et al. \\
1985
\end{tabular} & $\begin{array}{l}\text {-Biopsies } \\
\text { from the } \\
\text { vastus } \\
\text { lateralis of } 16 \\
\text { well trained } \\
\text { men } \\
\text {-17-32 y.o. } \\
\text {-Groups: }\end{array}$ & $\begin{array}{l}\text { - Control }=25 \\
\text { nm diameter } \\
\text {-Pale and } \\
\text { rudimentary } \\
\text { shape of the } \\
\text { subsarcolem } \\
\text { mal glycogen } \\
\text { particles post }\end{array}$ & $\begin{array}{l}\text { Resting control: } \\
\text { - Large subsarcolemmal glycogen particles } \\
\text { accumulation } \\
\text { - Large inter-myofibrillar glycogen particles } \\
\text { accumulation } \\
\text { - Intra-myofibrillar. rows of single glycogen } \\
\text { particles oriented longitudinally between the thin } \\
\text { or thick myofilaments on either Z-line }\end{array}$ \\
\hline
\end{tabular}




\begin{tabular}{|c|c|c|c|}
\hline & $\begin{array}{l}\text { 1. Resting } \\
\text { control }(n=4) \\
2.42 \mathrm{~km} \text { run } \\
(n=6) \\
3.20 \times 25-\mathrm{sec} \\
\text { treadmill } \\
\text { sprints }(n=6) \\
\text {-Biopsy was } \\
\text { obtained } \\
\text { approximately } \\
45 \text { minutes } \\
\text { post-exercise }\end{array}$ & sprint & $\begin{array}{l}\text { Sprint exercise: } \\
\text {-Large } \downarrow \text { in subsarcolemmal glycogen, and the } \\
\text { particles remaining have a pale and rudimentary } \\
\text { shape } \\
\text { - Myofibrillar. dominant loss of I band glycogen } \\
\text { (intra-myofibrillar), while accumulation of } \\
\text { glycogen were still present around the } \\
\text { mitochondria } \\
\text {-Particles of lesser density were commonly found } \\
42 \mathrm{~km} \text { run: } \\
\text {-Barely any detectable glycogen particles in the } \\
\text { core of the fibres were regularly observed } \\
\text { - The subsarcolemmal glycogen was chiefly } \\
\text { unaffected in depleted fibres, and the particles in } \\
\text { that location retained their size and shape } \\
\text {-They semi-quantified the subsarcolemmal } \\
\text { glycogen distribution by \# of glycogen clusters } \\
\text { per } 50 \mu \mathrm{m} \text { stretch of fibre after both type of } \\
\text { exercise: it was } 20 \pm 3 \text { after the } 42 \mathrm{~km} \text { run and } 6 \\
\pm 2 \text { after the sprints (no control) }\end{array}$ \\
\hline $\begin{array}{l}\text { Friden et al. } \\
1989\end{array}$ & $\begin{array}{l}\text { Biopsies } \\
\text { from the } \\
\text { vastus } \\
\text { lateralis of } 8 \\
\text { men } \\
\text {-22-36 years } \\
\text { old } \\
\text {-Groups: } \\
\text { 1. Resting } \\
\text { control }(n=5) \\
2.60 \times 8 \text {-sec } \\
\text { cycling sprints } \\
(n=3)\end{array}$ & $\begin{array}{l}\text { Resting } \\
\text { control } \\
\text { Two separate } \\
\text { populations of } \\
\text { particles were } \\
\text { distinguished } \\
\text { in the } \\
\text { myofibrillar } \\
\text { locations: } \\
\text {-Heavily } \\
\text { stained } 18-30 \\
\text { nm diameter } \\
\text { particles } \\
\text {-Lighter } \\
\text { particles 45- } \\
60 \mathrm{~nm} \\
\text { diameter }\end{array}$ & $\begin{array}{l}\text { Resting control: } \\
\text {-Subsarcolemmal: Large focal clusters of } \\
\text { glycogen particles were found around the } \\
\text { mitochondria within this space. The clusters } \\
\text { occurred irregularly but always in proximity to the } \\
\text { mitochondria } \\
\text {-Inter-myofibrillar: Glycogen accumulation mostly } \\
\text { found at the level of the I band, although these } \\
\text { clusters frequently extended over the length of } \\
\text { several sarcomeres. } \\
\text {-Intra-myofibrillar: } \\
\text {-Para-Z-disc distribution: rows of single particles } \\
\text { oriented longitudinally between the thin filaments } \\
\text { at either side of the Z-line. The rows occupied a } \\
\text { region bordered by the Z-line and extending } 350- \\
450 \text { nm. } \\
\text { - } N_{2} \text {-line location: rows of two or three particles } \\
\text { were detected at the lateral end of the I band (i.e. } \\
N_{2} \text {-line). The distance between these particles } \\
\text { was approximately equal to the diameter of one } \\
\text { particle ( } 25 \text { nm) } \\
\text { - H-zone location: infrequent occurrence of single } \\
\text { row of particles or single particles flanking the } \\
\text { bare region of the myofibrillar } M \text {-band. } \\
\text { Sprint exercise: } \\
\text {-Subsarcolemmal deposits were of lesser } \\
\text { magnitude compared to control, due to both a } \\
\text { smaller concentration of particles and weaker } \\
\text { staining characteristics of individual particles } \\
\text { - Glycogen particles were only occasionally found } \\
\text { at the A band, and then solely inter-myofibrillar. } \\
\text { o No particles were seen at the N2-line } \\
\text { olrregular rows of particles were infrequently } \\
\text { found at the Para-Z-disc }\end{array}$ \\
\hline
\end{tabular}


As discussed previously, skeletal muscles are composed of different fibre types, each with different physiological and metabolic properties. To address potential fibre type specific characteristics of the subcellular glycogen distribution, some of the authors mentioned above also examined the different fibre types. Schmalbruch and Kamieniecha (1974) reported that, in resting skeletal muscles, the glycogen granules are located mainly between myofilaments in type I fibres, and mainly between myofibrils in type IIA and IIB fibres. Sjostrom and co-workers (1982) aiso found the intra-myofibrillar pattern of glycogen distribution to differ between fibre types. The reported that in type I fibres, the intra-myofibrillar rows are more numerous in the 1 band region, while in type IIB, they appeared more frequently in the A band. Furthermore, they reported a few particles in the $M$ band region, especially in type $\| B$ fibres. This latter finding was also reported as the only consistent difference between type I and II fibres by Friden and coworkers (1989). No further fibre type difference in resting skeletal muscle subcellular distribution has been detected in the available literature.

While these analyses are far from extensive, they provide a preliminary basis for the different subcellular locations of skeletal muscle glycogen. To summarise, the results of these few qualitative reports showed human skeletal muscle to be compartmentalised into three main subcellular locations, which include the subsarcolemmal space, the inter-myofibrillar space. and the intra-myofibrillar space. Of these, the inter-myofibrillar one is suggested to be the most quantitatively important. Both the inter- and intra-myofibrillar glycogen is predominately localised at the level of the myofibrillar I band. 
Effect of exercise on glycogen distribution.

It is well known that skeletal muscle cells utilise almost all of their glycogen to supply fuel for contractions. However, it is also established that different types of exercise create different metabolic and physiologic demands on the skeletal muscles. The impact of different types of exercise on the subcellular location of glycogen was therefore addressed by the group of Ekblom and co-workers (Friden et al. 1985 and 1989; Sjostrom et al. 1982). The results are summarised in Table 2.

Prolonged ( 30 and $42 \mathrm{~km}$ ) moderate-intensity exercise was demonstrated to lead to the preferential depletion of type I over type II fibres (Friden et al. 1985; Sjostrom et al. 1982). An extensive depletion of glycogen in the core of the depleted fibres was regularly observed, and the particles remaining were of smaller sizes. In contrast, shorter rows of normal size particles located in the I band were relatively frequent (intramyofibrillar glycogen), demonstrating a relative sparing of the glycogen located in this subcellular location (Sjostrom et al. 1982). Interestingly, the subsarcolemmal glycogen was found to be extensively depleted in one study (Sjostrom et al. 1982), and relatively less utilised in the other study, where the particles in that location were reported to retain their size and shape (Friden et al. 1985).

On the other hand, following $20 \times 25$-second or $60 \times 8$-second sprint exercises, both type I and II fibres were found to be depleted (Friden et al. 1989; Sjostrom et al. 1982). A large subsarcolemmal-biased glycogen depletion was seen, causing both a smaller concentration of particles and weaker staining characteristics of individual particles in that space (Friden et al. 1989; Sjostrom et al. 1982). Similarly, an extensive intra-myofibrillar glycogen depletion was observed (Sjostrom et al. 1982) in all three intra-myofibrillar sub-compartments (Friden et al. 1989). In contrast, the intermyofibrillar glycogen was found to be relatively less affected by these intense exercises, and post-exercise accumulations of glycogen were still present around the mitochondria. 
The information provided by these few investigations is extremely interesting. However, they should be interpreted with caution given the qualitative nature of the description. In an attempt to add a quantitative component to their analysis, Friden and co-workers (1985) actually semi-quantified the subsarcolemmal glycogen depot following either an intense sprint exercise $(20 \times 25$ seconds) or a $42-\mathrm{km}$ run. Their procedure involved counting the number of subsarcolemmal glycogen clusters along a $50 \mu \mathrm{m}$ stretch of fibre. Their results showed the number of subsarcolemmal glycogen clusters per $50 \mu \mathrm{m}$-stretch of muscle fibre to be $20 \pm 3$ and $6 \pm 2(p<0.001)$, for the moderate and intense exercises, respectively. Based on these results, Friden and colleagues (1985 and 1989) proposed that the subsarcolemmal glycogen particles could be stored there to secure energy yield for the maintenance of the plasma membrane potential and I or to act as an intra-cellular reserve energy supply. Accordingly, they proposed that during high-intensity exercise, the circulating energy pools are not sufficient for both the piasma and contraction functions. Therefore, the subsarcolemmal glycogen stores would be utilised to yield ATP for the $\mathrm{Na}^{*} / \mathrm{K}^{+}$pump (Friden et al. 1985 and 1989). A quantitative approach is required to verify these qualitative findings.

In summary, these preliminary ultrastructural analyses provide essentially novel information concerning a compartmentalised pattern of glycogen distribution. These data are in line with the emerging biochemically-based concept of the subcellular organisation of the enzymatic machinery and metabolic pathways into an integrated compartmentalised glycogen metabolism (see section "Glucose disposal and glycogen metabolism in skeletal muscles"). A quantitative approach is however necessary to confirm and extend these qualitative results. 


\section{Conclusion}

This review presented an overview of the literature concerned with various aspects of skeletal muscle glycogen and its metabolism. Numerous and extensive biochemical experiments have provided the basis of our understanding of glycogen metabolism. Mathematical modelling has provided guidance and hypotheses to stimulate research in the field of glycogen metabolism. Findings from morphological and ultrastructural experiments, although limited by their qualitative nature, have provided invaluable information about different aspects of glycogen and its metabolism, which are not feasible with standard biochemical techniques. While substantial knowledge has been obtained by the combination of results from these different research fields, many questions remain. Is the Whelan's model of glycogen structure currently accepted truly representative of reality? What are the respective mechanisms by which insulin and exercise induce GLUT-4 translocation to the transverse tubular system? Given the role of glycogen in whole body glucose homeostasis, could enhancing glycogen storage in skeletal muscle of insulin resistant individuals help improve insulin resistance and the associated morbidity? Are pro- and macro-glycogen truly two distinct metabolic entities and is pro-glycogen an intermediate in the biosynthesis of glycogen? By which mechanism is glycogen concentration controlling practically all pathways of glycogen metabolism? Does the translocation of glycogen synthase involve the migration of the entire glycogen-protein complex or are individual proteins organised into effective assemblies at the sites of synthesis? How does the direct control of enzyme activities relate to the positional controls in regulating metabolic rates? How is the glycogen turnover regulated during intense exercise? Is the subcellular distribution of glycogen a key player in the regulation of glycogen metabolism? What is the specific role of the glycogen in each subcellular location in the different types of exercise? Is the role of the 
subsarcolemmal glycogen truly to provide energy for the $\mathrm{Na}+\mathrm{K}+\mathrm{ATPase}$ at the cellular membrane? Is the compartmentalisation of glycogen and its metabolising enzymes the key to the specificity and control of each pathways of glycogen metabolism? The development of new technology will most likely contribute to answering most of these yet unanswered questions. 
Chapter III

Statement of the hypotheses 
The preceding review of literature emphasised the emerging concept of a key role for subcellular translocation of enzymes and compartmentalisation of different metabolic pathways in the control of glycogen metabolism. Over the past 150 years, numerous investigations have employed different biochemical approaches to examine many aspects of glycogen metabolism. These provided the basis of our understanding in this area. On the other hand, limited investigations have addressed glycogen metabolism using a morphological or ultrastructural approach. Furthermore, a major limitation to the generalisation of the information provided by these few investigations relates to the qualitative nature of their experimental designs. This probably is caused by the time-consuming nature of this type of analysis, as well as the limits imposed by the precision of the equipment. While the results of the preliminary studies are interesting, the methodological approach used in estimating muscle glycogen concentration lacks the objectivity of quantitative procedures. The selection of fibres and subcellular locations has not been systematic, and the sample sizes have been restricted owing to the labour-intensive nature of TEM work and the lack of advanced computerised processes to assist in data collection and analysis. Nevertheless, the available information illustrates distinct subcellular locations of glycogen particles and results suggest that these locations represent metabolic pools that have specific metabolic roles and regulations. The results also demonstrate the great potential of TEM in investigating glycogen metabolism. With the development of powerful computer software, the ability to develop quantitative techniques is now feasible. The use of TEM procedures offers many specific advantages over traditional biochemical techniques, including principally the ability to discriminate between the different subcellular compartments and to determine the pattern of glycogen concentration in terms of particle number and size. Such information will be critical in understanding the roles of 
the subcellular compartmentalisation pattern of glycogen particles as potential regulatory control mechanisms of carbohydrate metabolism in skeletal muscles.

The primary objective of the present study was therefore to develop and validate a TEM-based technique to quantify the subcellular distribution of human skeletal muscle glycogen (Chapter IV - Detailed methods). This technique was then utilised to examine and quantify the characteristics of the subcellular distribution of skeletal muscle glycogen in human resting samples (Chapter V - Experiment 1). Subsequently, the technique was employed to quantify the pattern of glycogen repletion during the 48 hours following a moderate-intensity glycogen-depleting exercise (Chapter VI Experiment 2). Finally, we quantified the pattern of glycogen depletion during a highintensity sprint exercise (Chapter VII - Experiment 3). For every experimental condition, the characteristics of the glycogen distribution were examined with respect to particle sizes, number and concentration (volume), and compared between subcellular locations and fibre types.

We hypothesised that (1) the distribution of glycogen in human skeletal muscle, with respect to particle size, number and concentration (volume), is heterogeneous in terms of subcellular locations and fibre types, (2) the frequency distribution of glycogen particle size follows a normal distribution, and (3) the pattern of glycogen depletion during exercise, as well as that of glycogen repletion following exercise is compartmentalised. Based on the lack of extensive literature, we present the following research questions: (a) will the pro- and macro-glycogen concentration correspond to the frequency distribution of particle sizes? (b) will glycogen depletion and repletion be characterised by a decrease in glycogen particle size, number or both? (c) will the glycogen degradation during and the glycogen synthesis following exercise be 
metabolised from specific subcellular locations? (d) will the glycogen in the subsarcolemmal space be used primarily to preserve membrane potential? The present thesis will address these hypotheses and research questions. 


\section{Chapter IV}

\section{Detailed Methods}


The following description refers to the elaboration of a transmission electron microscopy (TEM) based technique developed to quantify the content and the distribution of subcellular skeletal muscle glycogen in human. The technique was applied to biopsy samples from human skeletal muscle obtained under different experimental conditions. The exact experimental protocol involved in examining each specific condition is described in the appropriate chapter, and therefore, will not be covered here. The present description outlines the procedures used after obtaining skeletal muscle samples by percutaneous needle biopsy technique.

Resting muscle biopsies were obtained from the vastus lateralis of the subjects by use of the percutaneous needle biopsy technique (Hultman 1967) with suction modification (Evans 1984). Each muscle sample was divided into two portions, one for biochemical and one for TEM analysis. Two blinded independent investigators performed the biochemical and histochemical analyses, and each type of analysis was performed by the same investigator for all samples.

\section{Biochemical analysis}

A piece of each muscle biopsy was immediately placed in liquid nitrogen and subsequently stored at $-80^{\circ} \mathrm{C}$ until it was freeze-dried and dissected free of visible blood, connective tissue, and other non-muscle elements. A 1.5- to 3.5-mg portion of freeze-dried muscle was extracted in $1.5 \mathrm{mM}$ perchloric acid and analysed for pro- and macroglycogen (Adamo and Graham 1998a). Enzymatic measurement of glucosyl units (Bergmeyer 1974) was then performed, and reported as millimoles of glucosyl units per kilogram of dry muscle weight ( $\mathrm{mmol} \times \mathrm{kg} \mathrm{dw}^{-1}$ ). 


\section{TEM analysis}

The specimen preparation for electron microscopy can be summarised in five main steps: 1) Fixation (primary and secondary); 2) Dehydration; 3) Infiltration with resin; 4) Cutting; and 5) Staining.

Following the percutaneous muscle biopsy, approximately $1.5 \mathrm{~mm}^{2}$ of the fresh muscle tissue was immediately fixed in $2.0 \%$ glutaraldehyde in $0.1 \mathrm{M}$ sodium cacodylate buffer. Glutaraldehyde is the most commonly used primary fixative in electron microscopy (Maunsbach and Afzeliuz 1999). It is a five carbon compound with terminal aldehyde groups, which allow the molecule to cross-link proteins. In particular, the aldehyde groups react with the $\alpha$-amino groups of lysine residues of adjacent proteins, thereby cross-linking them. The reaction also likely involves other amino acids, as well as carbohydrates.

Within 24 to 48 hours, the samples were post-fixed in a solution of $1 \%$ osmium tetroxide $(\mathrm{Os})$ and $1.5 \%$ potassium ferricyanide $\left(\mathrm{K}_{4} \mathrm{Fe}(\mathrm{CN})_{6}\right)$ in $0.1 \mathrm{M}$ sodium cacodylate. This secondary fixation, using a combination of $\mathrm{Os}$ with $\mathrm{K}_{4} \mathrm{Fe}(\mathrm{CN})_{6}$, enhances the contrast-staining of intra-muscular glycogen (Hayat 1989). Compared to Os alone, the $\mathrm{Os}-\mathrm{K}_{4} \mathrm{Fe}(\mathrm{CN})_{6}$ mixture yields an enhanced electron-scattering capacity of many cellular components, including intra-muscular glycogen, while reducing the electron-scattering capacity of ribosomes and chromatin. Because ribosomes have a similar order of magnitude of size (10 to $15 \mathrm{~nm}$ ) as do glycogen particles, this technique also decreases the relative optical density of ribosomes compared to glycogen, and therefore helps to distinguish between these two cellular components (Revel 1960). This combination therefore facilitates the analysis of intra-muscular glycogen by enhancing its contrast compared to other cellular components. Indeed, an analysis of the influence of sample processing methods in electron microscopy on the ultrastructure of glycogen particles 
was recently performed in animal tissues (Afonso 2000). The results confirmed that, compared to other preparations, the highest electron density of glycogen granules was obtained when osmium tetroxide fixative was supplemented with potassium ferricyanide. Furthermore, the value of $\mathrm{K}_{4} \mathrm{Fe}(\mathrm{CN})_{6}$ as a sample preparation technique to visualise glycogen particles has been examined and confirmed by Robinson and co-workers (1982).

The samples were then dehydrated in graded ethanol and subsequently infiltrated with graded mixtures of propylene oxide and Spurr's resin. After primary and secondary fixation, the water in the muscle cells is replaced with a fluid, which acts as a solvent between the aqueous environment of the cell and the hydrophobic embedding media. Ethanol is one of the most commonly used dehydrating agents and solvent for this purpose (Maunsbach and Afzeliuz 1999). The tissues were then placed in increasing concentrations of ethanol until the tissue was in absolute ethanol. At this point, the tissue was placed in propylene oxide, which is highly miscible with the plastic embedding medium (Spurr's resin). Propylene oxide also further dehydrates the tissue. The propylene oxide was then gradually replaced by Spurr's resin by placing the tissue into increasing concentrations of resin / propylene oxide mixture, until pure resin was reached.

Thin sections $(70 \pm 10 \mathrm{~nm})$ were then cut, using ultramicrotomy, placed on 200 mesh copper grids, and for each sample, some sections were stained with uranyl acetate and lead citrate. After careful evaluation, it was concluded that the uranyl acetate and lead citrate staining were detrimental to our glycogen analysis, and unstained sections were used for analysis. The non-specific nature of the double chemical staining induced by uranyl acetate and lead citrate renders essentially all cellular components considerably electron dense (Hayat 1989), and hence reduces the contrast between glycogen particles and other cellular structures. 
Finally, the sections were examined and photographed in a JEOL 1200 EX electron microscope. For each session of electron microscopy, a standard calibration grid (cross grating replica from SPI Supplies West Chester PA.; 2160 grid lines per mm) was used to calculate the exact magnification.

A total of 5-6 muscle fibres were photographed from each muscle biopsy sample (i.e. each thin section). During the selection process performed by the main investigator, special attention was paid in order to sample at least one fibre of each fibre type (I, IIA, and IIB). The criteria used for this preliminary fibre selection were gross morphological characteristics including Z-line width, lipid content, shape and content of the mitochondria, and SR and T-tubule appearance (Landon 1992). This was done in order to allow fibre type comparison during the subsequent quantitative analysis.

For each of the 5-6 fibres per muscle biopsy sample, a total of 12 images were obtained. The first image was a general view of the fibre at a magnification of $3000 \times$ to facilitate the process of fibre typing (see "Fibre typing" section below). The remaining images were obtained at $20000 \times$ for the subcellular glycogen distribution analysis. Figure 3 illustrates the protocol used for the selection of the 11 systematic sampling regions (images) used for glycogen distribution analysis within each fibre.

The areas selected as images represented three different subcellular locations: (1) subsarcolemmal space, (2) "superficial" myofibrils, and (3) "deep" myofibrils. This selection of different locations for the images (subsarcolemmal, superficial and deep myofibrillar) was based on previous suggestions that skeletal muscle glycogen is heterogeneous, and that its pattern of distribution and utilisation may vary according to different intra-muscular subcellular locations (Friden et al. 1985 and 1989; Schmalbruch and Kamieniecka 1974). Accordingly, in the present work, the purpose of obtaining a representative sample of images from each of the three subcellular locations mentioned 
above was to allow the quantitative comparison of the different glycogen variables between them.

For a given fibre (Figure 3), images $\mathbf{S 1}$ to $\mathbf{S 5}$ comprised portions of the subsarcolemmal space: (S1) one pole of a nucleus; (S2) between the sarcolemma and a nucleus; (S3) between a nucleus and the myofibrils; (S4) sarcolemma adjacent to a cluster of mitochondria; and (S5) sarcolemma lacking mitochondria. These sites were selected to gain representation of glycogen particles with respect to their association with the two main subsarcolemmal organelles, the nuclei and mitochondria. Although together they are not quantitatively representative of the subsarcolemmal space (since the glycogen and the organelles are heterogeneously distributed within the subsarcolemmal space and the area examined is only a relatively small area of the overall muscle fibre), they represent a consistent, standardised measure of the subsarcolemmal space, and were used for comparison between experimental conditions. Figures 4 to 8 illustrate an example of the five different images that were used to represent the subsarcolemmal space. 
Nucleus

S2

S3
S1

\section{Sarcolemma}

M5

M4

M2

M6

M3

S5

\section{Sarcolemma}

Figure 3. Photograph of a human muscle fibre $(3000 \mathrm{X})$ illustrating the systematically random image selection protocol. "M1, M3, and M5" represent the superficial myofibrillar images. "M2, M4, and M6" represent the deep myofibrillar images. "S1 to S5" represent the subsarcolemmal images. 


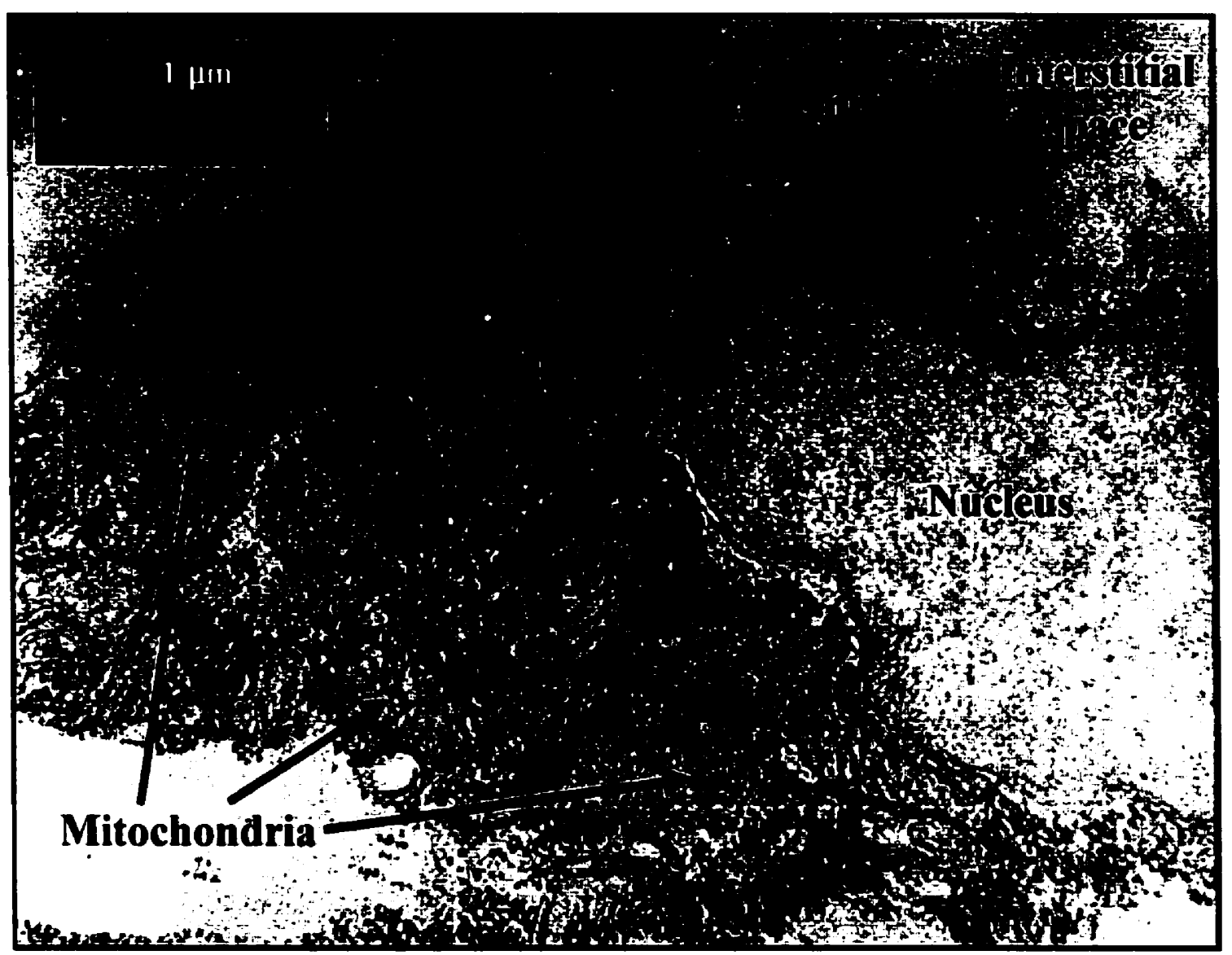

Figure 4. Illustrates the first subsarcolemmal location (S1) selected for the analysis. It represents the space located just beneath the sarcolemma, adjacent to a nucleus. For standardisation of this particular type of subsarcolemmal image, the nucleus must represent approximately $50 \%$ of the width of the image. When relevant, that is when the size of the nucleus is small enough, the first myofibril (closest to the sarcolemma) and the corresponding first inter-myofibrillar space (but not the second myofibril) are included in the analysis. 


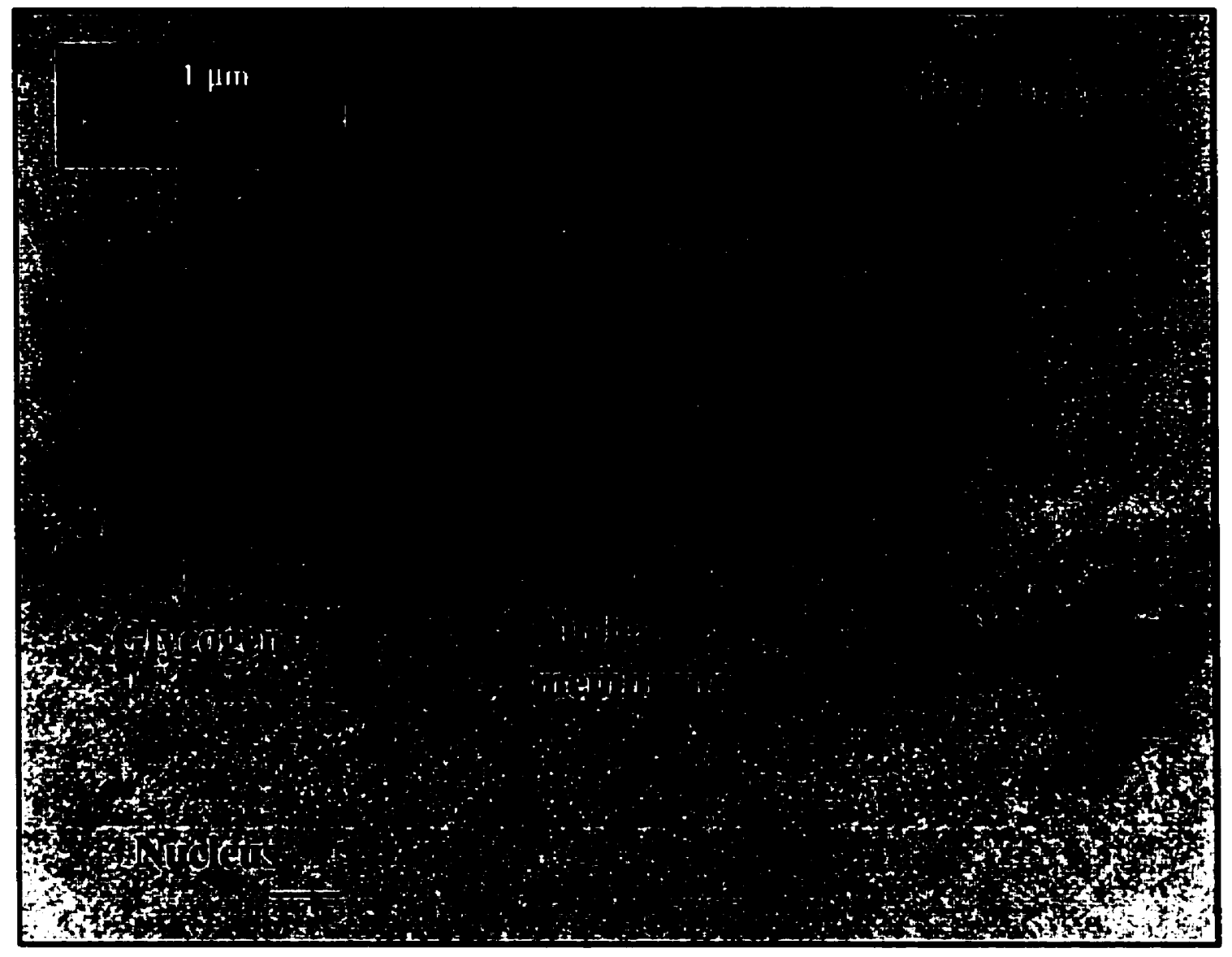

Figure 5. Illustrates the second subsarcolemmal location (S2) selected for the analysis. It represents the space between the sarcolemma and the nuclear membrane. No myofibrillar tissue (contractile tissue) is included in this subsarcolemmal location. 
$1 \mu \mathrm{II}$

Figure 6. Illustrates the third subsarcolemmal location (S3) selected for the analysis. It represents the space between the nucleus and the myofibrils. For standardisation of this particular type of subsarcolemmal image, the first myofibril, adjacent to the sarcolemma, and the corresponding first inter-myofibrillar space (but not the second myofibril) are always included in the analysis. 


\section{$1 \mu \mathrm{mII}$}

Figure 7. Illustrates the fourth subsarcolemmal location (S4) selected for the analysis. It represents the space adjacent to a section of sarcolemma in which mitochondria are abundant. For standardisation of this particular type of subsarcolemmal image, the first myofibril, adjacent to the sarcolemma, and the corresponding first inter-myofibrillar space (but not the second myofibril) are always included in the analysis. 
Figure 8. Illustrates the fifth subsarcolemmal location (S5) selected for the analysis. It represents the space adjacent to a section of sarcolemma deprived of mitochondria. For standardisation of this particular type of subsarcolemmal image, the first myofibril, adjacent to the sarcolemma, and the corresponding first inter-myofibrillar space (but not the second myofibril) are always included in the analysis. 
Images M1 to M6 were used to evaluate the myofibrillar space: M1, M3, and M5 represent myofibrils located immediately adjacent to the subsarcolemmal space ("superficial" myofibrillar space); M2, M4, and M6 were for analysis of myofibrils located towards the centre of the muscle fibre ("deep" myofibrillar space). Therefore, a total of six images represented the myofibrils: three in a superficial region and three in a deep region (Figure 3).

A preliminary analysis was performed in order to determine the minimal number of images necessary to represent the myofibrillar space of one whole fibre. This analysis examined the change in the coefficient of variation (standard deviation $\times 100 /$ mean) obtained for the dependent variables (discussed below), as more images were added to the analysis (White 1987). The addition of images favours a decreased variation and, hence, depicts data more representative of the muscle fibre as a whole. The coefficient of variation for the dependent variables decreased as the sampling increased from one to six images. There was no further decrease in the coefficient of variation with the addition of seven to ten images. On this basis, 6 myofibrillar images were analysed (see Appendix A).

To summarise, 5-6 fibres were selected from each muscle biopsy. From each of these muscle fibres, 12 images were taken. One of these images $(3000 \mathrm{X})$ was used only to facilitate fibre typing. The remaining 11 images ( $20000 \mathrm{X}$ ) were further analysed for glycogen content and represented five subsarcolemmal spaces, three groups of superficial myofibrils, and three groups of deep myofibrils (total of six groups of myofibrils). Therefore, 11 images for each of 5-6 fibres were photographed, digitised, and analysed for each muscle biopsy sample, for a total of 55 to 66 (20000 X) images per biopsy sample. 


\section{Digitising.}

The images (in the form of negatives) were digitised using a cooled CCD camera and an Epi-illumination Darkroom system (UVP, Upland, California). Each negative was digitised as two equal half images in order to facilitate the analysis (simply by approximately doubling the magnification). A total of 22 images (11 negatives at 20000 $X$ times 2 images) were therefore used to represent each muscle fibre. The software used for digitising purposes was "Labview $\otimes$ ", and a description of the relevant settings is presented in Appendix B.

Analysis of subcellular glycogen content and distribution.

The analysis of intra-muscular glycogen distribution was carried out using the image analysis software Image Pro Plus $\otimes($ version 4.0). The analysis was conducted in three parts: (1) single particle evaluation, (2) total glycogen, and (3) intra- and intermyofibrillar glycogen. Following is a complete overview of each part of the analysis. The calculations are described in detail later in the "calculation" section.

(1) Single particle glycosome analysis:

The single particle analysis consisted of isolating the particles that were not clustered, and determining the average single particle diameter (particle diameter), area (particle area) and volume (particle volume). Both, particle diameter and area, were directly and independently measured by the image analysis software (Image Pro Plus (8) version 4.0). The particle volume was calculated (see calculation section). These single particle variables were then compared between time (except in the first experiment where time was not included as a variable), subcellular locations (subsarcolemmal space, superficial myofibrils, and deep myofibrils), and fibre types (I, IIA, and IIB). The following is a detailed explanation of the single particle analysis procedure: 
- The mean particle diameter, area and volume were determined for each image independently by averaging all the particles for that image.

- For each fibre, the mean diameter, area and volume for the superficial myofibrils were determined by averaging all the particles from images $M 1, M 3$, and $M 5$ (see Figure 3), as opposed to averaging the mean values of each of these images. This allowed taking into account the number of particles in each individual image, and was therefore more representative of the mean diameter, area and volume in the myofibrils of interest.

- For each fibre, the mean diameter, area and volume for the deep myofibril were determined from images M2, M4, and M6 (see Figure 3), as for superficial myofibrils.

- For each fibre, the mean diameter, area and volume for the subsarcolemmal space was determined from images $\mathrm{S} 1$ to S5 (see Figure 3), as for superficial and deep myofibrils. By convention and to standardise the procedure, the particles located within the first myofibril and the first inter-myofibrillar space (but not the second myofibril) adjacent to the sarcolemma were included in all subsarcolemmal space images (except for image $\$ 2$ which represented the space between the sarcolemma and the nucleus). This corresponds to a shell of approximately $1 \mu \mathrm{m}$ from the sarcolemma, and has previously been considered as the "subsarcolemmal outer annulus" (Eisenberg and Kuda 1975). While this procedure is likely to affect the results of the comparison between subcellular locations, it will minimise the risk of incorrectly finding a statistically significant difference.

- For each fibre, the mean diameter, area and volume for the entire fibre was determined by averaging all the particles from images $M 1$ to $M 6$ and $S 1$ to $S 5$ (see Figure 3), as opposed to averaging the mean values of each of these images. This 
overall fibre value was used in the time and fibre type comparisons of single particle diameter, area and volume.

(2) Total glycogen analysis:

The total glycogen analysis consisted of determining both the total area (glycogen area) and volume (glycogen volume) of the image consisting of glycogen, as well as the number of glycogen particles that constituted this area and volume (\# of glycogen particles). The total glycogen area was directly measured by the image analysis software (Image Pro Plus $\otimes$ version 4.0). The total glycogen area was then converted to a total glycogen volume (see calculation section). The number of glycogen particles was calculated from the total glycogen area and the single particle variables (see calculation section). This total glycogen analysis was necessary since "single particle analysis": (1) only includes the glycogen particles that were isolated enough to be considered individually and therefore, excluded all the clusters of glycogen particles; (2) could not calculate the total number of glycogen particles for each image since it did not include all the glycogen present in the image. These total glycogen variables were then compared between time (except in the first experiment where time was not included as a variable), subcellular locations (subsarcolemmal space, and superficial and deep myofibrils) and fibre types (I, $\| A$, and $\| B$ ). Following is a detailed explanation of the total glycogen analysis procedures:

- The total glycogen area was determined (Image Pro Plus $\otimes$ version 4.0) and the total glycogen volume and total \# of glycogen particles were calculated (see calculation section) for each individual image (including the images from the subsarcolemmal space, and superficial and deep myofibrils). 
- For each fibre, the mean total glycogen area, volume and \# of glycogen particles for the superficial myofibrils were determined by averaging the values from individual images M1, M3, and M5 (see Figure 3).

- The same was done for the deep myofibrils (M2, M4, and M6) and the subsarcolemmal space S1 to S5 (see Figure 3).

- As for the single particle analysis, based on the definition of the subsarcolemmal space found in the literature (Eisenberg and Kuda 1975), the first myofibril and the first inter-myofibrillar space (but not the second myofibril) adjacent to the sarcolemma were included in the subsarcolemmal space images. Again, and as discussed previously, while this procedure is likely to affect the results of the comparison between subcellular locations, it will minimise the risk of incorrectly finding a statistically significant difference.

- For each fibre, the overall total fibre glycogen area, volume and \# of glycogen particles were obtained by averaging the values from all deep and superficial myofibrillar images (average from images M1 to M6). Therefore, these overall total fibre values did not include the subsarcolemmal space values. The inability to obtain such a value for the total glycogen variables, which are all expressed per $\mu \mathrm{m}^{3}$ of muscle tissue, was due to not knowing the relative proportion of the muscle fibre space that is myofibrillar and subsarcolemmal. Hence, each subcellular location was expressed separately for the total glycogen variables, and the total myofibrillar value alone was used as a representation of the fibre. We recognise that this is a limitation and we address it in the "general discussion" section of the thesis.

- Therefore, for each fibre, a value for the total glycogen area, volume and \# of glycogen particles was determined for: (1) the superficial myofibrils; (2) the deep 
myofibrils; (3) the subsarcolemmal space; and (4) the overall fibre (which really represents the total myofibrillar value).

(3) Intra- and inter-myofibrillar glycogen analysis:

The intra- and inter-myofibrillar glycogen analysis consisted of dividing the amount of total glycogen of each myofibrillar image (deep and superficial) into its respective intra- and inter-myofibrillar sections. Hence, the intra- and inter-myofibrillar compartments were analysed separately. Figure 9 illustrates the difference between the intra- and inter-myofibrillar regions. The intra-myofibrillar glycogen was that which was located inside the myofibrils themselves. In contrast, the inter-myofibrillar glycogen was that which was located between two adjacent myofibrils. The sum of intra- and intermyofibrillar glycogen therefore corresponds to the total glycogen for a given myofibrillar image (deep or superficial). It is important to emphasise that this intra- and intermyofibrillar analysis was not relevant for the subsarcolemmal images, since the latter images only included one myofibril. Following is a detailed explanation of the intra- and inter-myofibrillar glycogen analysis procedures:

- The total glycogen analysis was first performed.

- For the images of the deep and superficial myofibrils (but not for the subsarcolemmal images since these only contain one myofibril), the glycogen that was located in the inter-myofibrillar space was subtracted from the total glycogen. By default, the glycogen remaining was named the "intra-myofibrillar glycogen".

- The "inter-myofibrillar glycogen" was then obtained by subtracting the intramyofibrillar glycogen from the total glycogen area. 
- Hence, for each of the deep and superficial myofibrillar images, the total, intra- and inter-myofibrillar glycogen variables (glycogen area, volume and \# of glycogen particles) were determined. In contrast, for the subsarcolemmal images, only the total glycogen variables were determined.

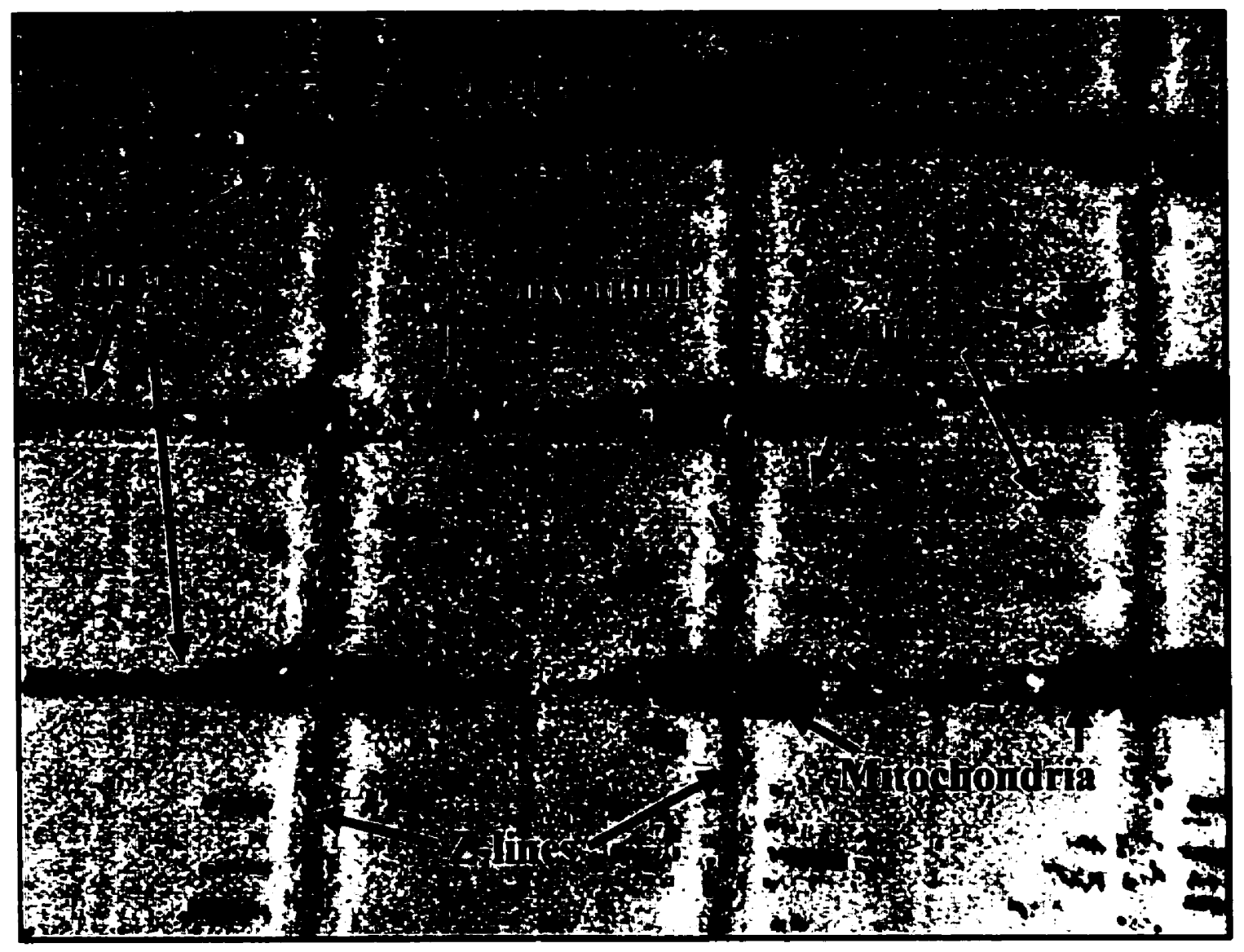

Figure 9. Illustrates the difference between inter-and intra-myofibrillar glycogen (labelled as Inter and Intra, respectively) $(\sim \times 20000)$. The inter-myofibrillar glycogen is defined as the glycogen located outside the myofibrils, that is, in between two adjacent myofibrils. The intra-myofibrillar glycogen is defined as the glycogen located within the myofibrils. In a given image, the sum of inter- and intramyofibrillar glycogen represents the total glycogen content of the image. 
Detailed computer analysis procedures.

Following the digitising process, a series of steps was standardised in order to minimise the possible biases in the subcellular glycogen distribution analysis.

(1) Preparing the images

(2) Cleaning the image

(3) Calibration

(4) Single particle analysis

(5) Glycogen content analysis

(6) Background analysis

(7) Z-line width measurement

(8) Image dimension measurement

(1) Preparing the image.

Because the level of darkness and contrast varied from negative to negative, it was necessary to prepare each image and make it suitable for the computer analysis. A copy of the Macro designed to execute this task is found in Appendix C.

Briefly, the Macro involved resizing the image $(85 \%)$, optimally equalising it (Image Pro Plus $(8)$ function), and inverting it. "Resizing" the image simply involved increasing the size of the original image to make it more suitable for further analysis. "Optimally equalising" involves achieving the best possible contrast distribution of pixel values in each particular image; this is a function of Image Pro Plus $®$ version 4.0. "Inverting" the image simply commands Image Pro Plus to reverse the image, that is to make the black pixels white and the white pixels black. This function was necessary since the images were digitised directly from the negatives and therefore were inverted compared to reality. Then, a spatial filter was applied to flatten the image (Flatten, Pass 
1, Strength 7). The "Flatten" function was repeated once or twice if required and depending on the image. The "Flatten" function was necessary to even out the background variations by reducing the intensity variations of the background pixels. This allowed enhancing the glycogen particles over the muscular tissue background.

Figures 10 and 11 illustrate examples of images before and after the preparation step, respectively.

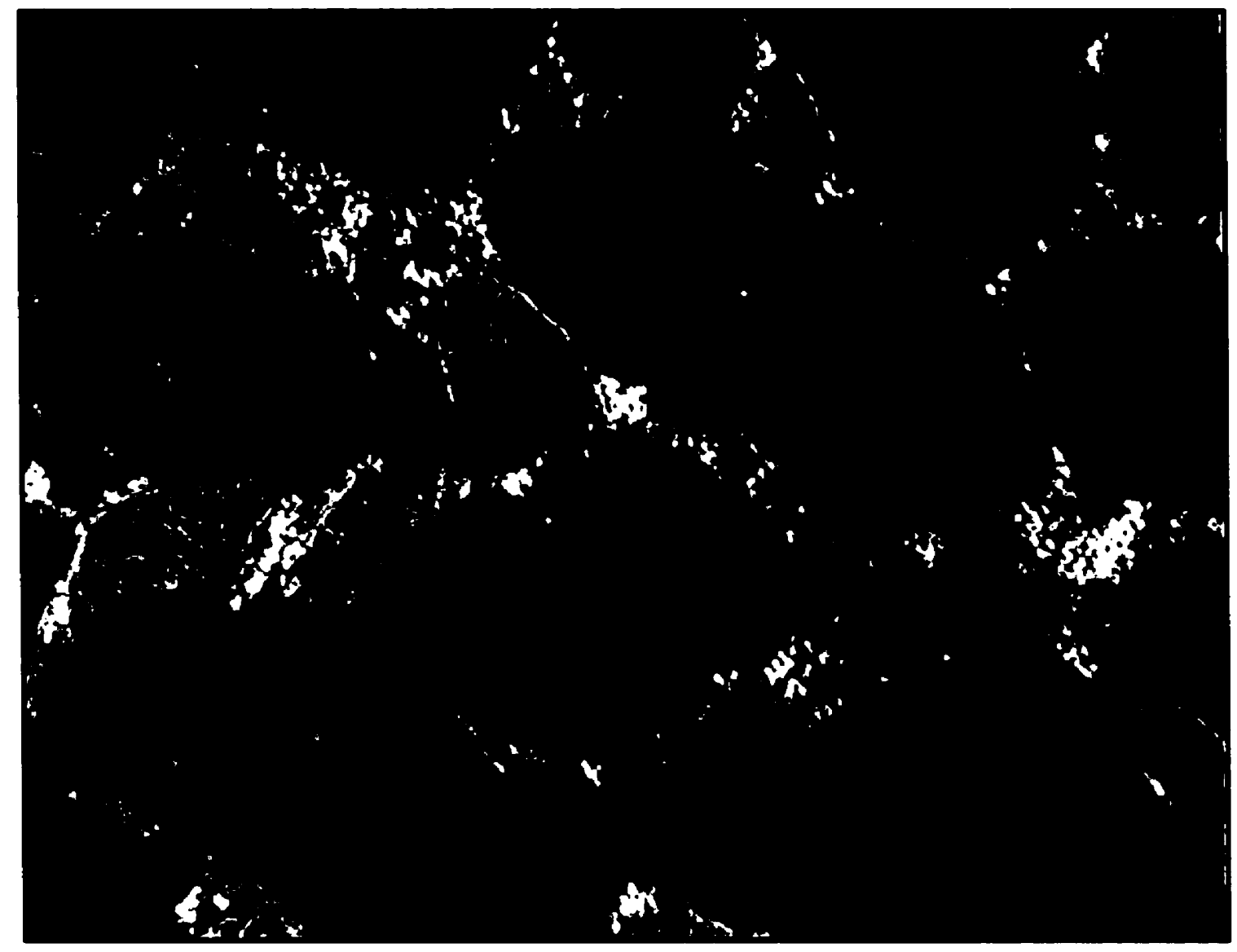

Figure 10. Illustrates a myofibrillar image immediately following digitisation ( $\times 20000)$. The image is digitised directly from the negative obtained through electron microscopy, which explains why the image is inverted from reality. 


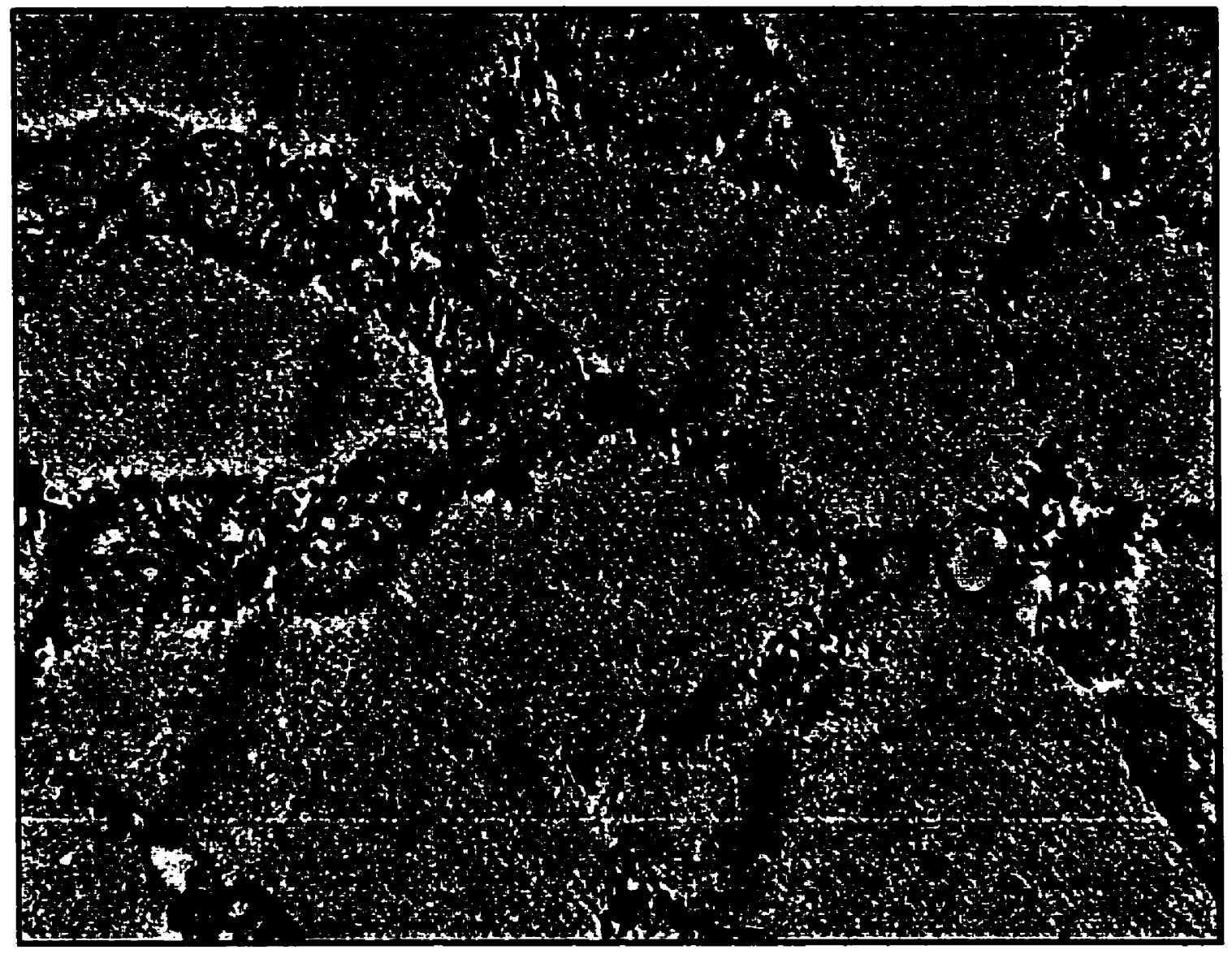

Figure 11. Represents the same image as Figure 10, but following its preparation $(-X 20000)$. This procedure was necessary to make the image suitable for the computer analysis. Refer to section "Preparing the images" for details on the procedure.

(2) Cleaning the image.

Because the fixation chemical used to enhance glycogen in the present study $\left(\mathrm{K}_{4} \mathrm{Fe}(\mathrm{CN})_{6}\right)$ also enhances membranes, certain organelles were accentuated along with the glycogen. Those include the mitochondria, the triads, the peroxisomes and the lysosomes, among others. It was therefore necessary to manually remove these organelles in order to isolate the glycogen. This phase of the analysis was extremely 
tedious and was performed by the same investigator for all samples. It consisted of manually framing the areas of interest (undesired material), and then converting them to absolute white. This completely eliminated them from the glycogen analysis. Figures 11 (see above) and 12 illustrate examples of myofibrillar images before and after the cleaning step, respectively.

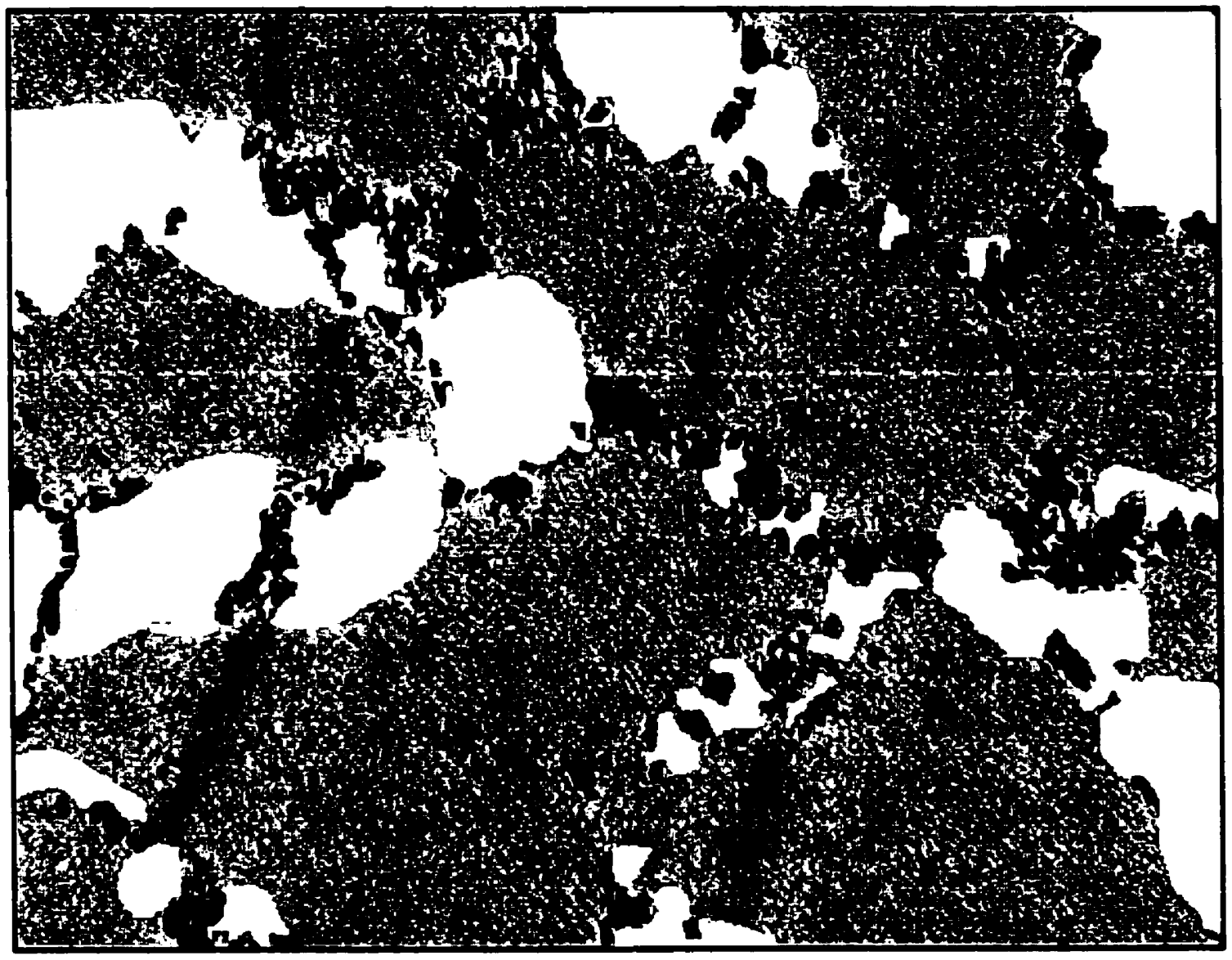

Figure 12. Represents the same image as Figure 11, but following the "cleaning" step of the analysis $(-X 20000)$. This procedure was necessary to eliminate the material, which given its density, could have been mistakenly considered as glycogen by the computer. Refer to section "Cleaning the image" for details on the procedure. 
In addition, in this step, it was necessary to prepare the subsarcolemmal images for the analysis. For the images from this particular subcellular location (subsarcolemmal), in addition to the presence of undesired organelles (which were present in all three types of subcellular locations), it was important to standardise the extent to which the myofibrils would be included. This was necessary since it was impossible to select subsarcolemmal images that included the same relative amount of myofibrils. It was therefore decided to standardise this by including in the subsarcolemmal space, the first complete myofibril and inter-myofibrillar space adjacent to the sarcolemma. As discussed above, this was based on the definition of subsarcolemmal space proposed by Eisenberg and Kuda (1975). This was done for every subsarcolemmal image analysed (except for the type $\mathbf{S 2}$ as described earlier). The remaining image, whether it consisted of the interstitial space or the myofibrils deeper to the one immediately adjacent to the sarcolemma, was disregarded from the analysis in the same manner as the undesired organelles (filled with white). Figures 13 and 14 illustrate an example of subsarcolemmal images before and after the cleaning step respectively. 


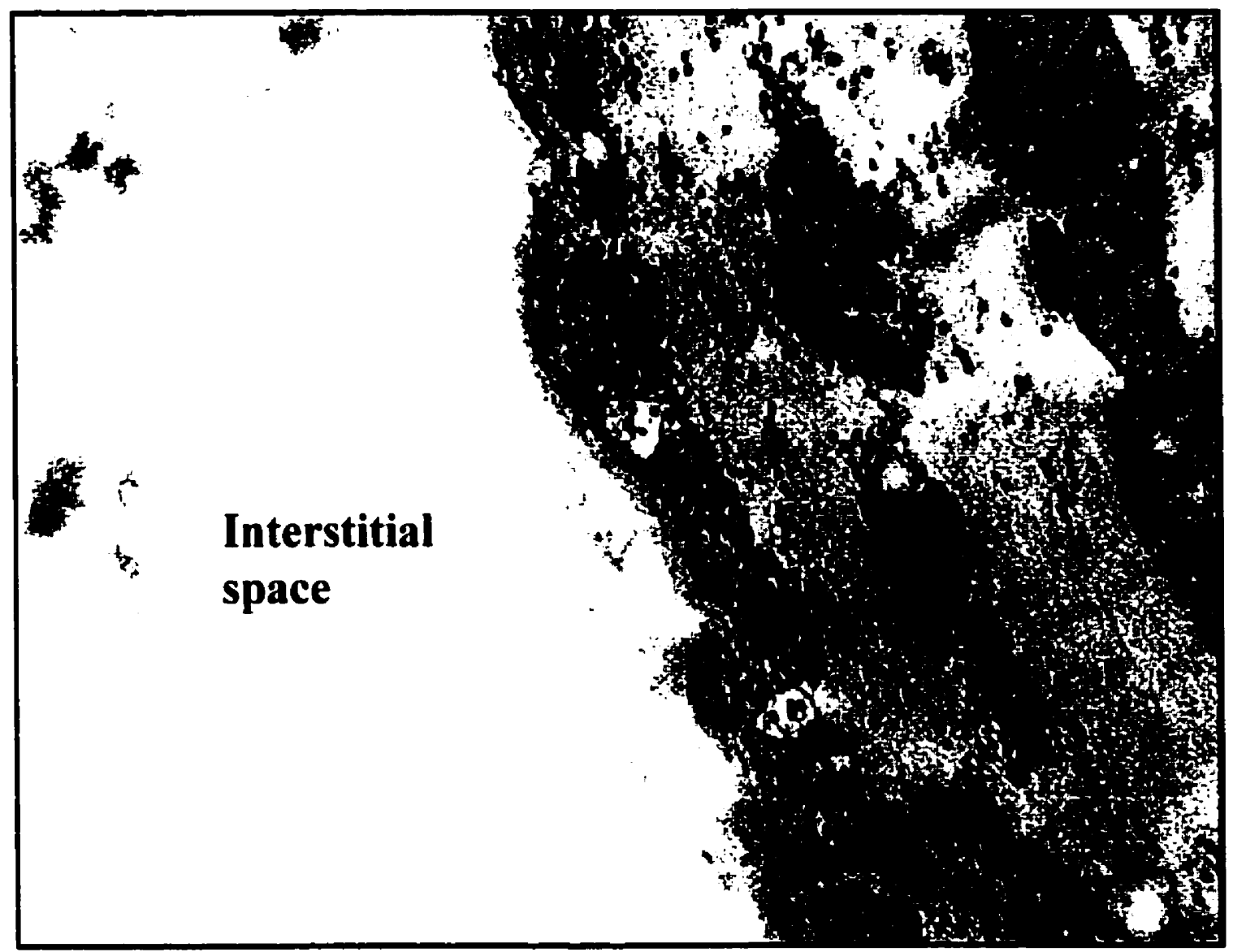

Figure 13. Illustrates an example of a subsarcolemmal image following the preparation of the image (image before preparation not shown) $(\sim \times 20000)$. As for myofibrillar images, this procedure was necessary to make the image suitable for the computer analysis. Refer to section "Preparing the images" for details on the procedure. 


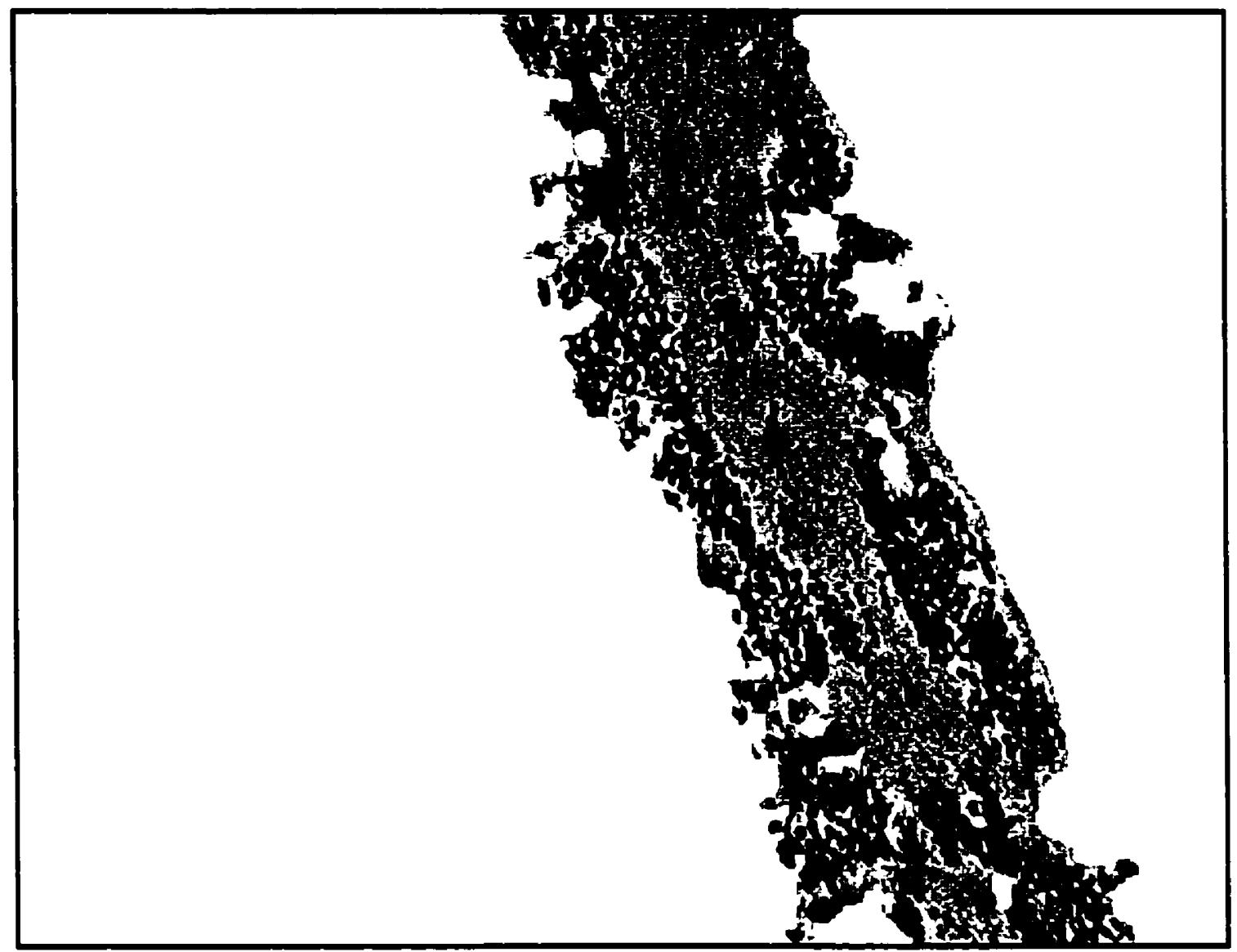

Figure 14. Represents the same image as Figure 13, but following the "cleaning" step of the analysis $(\sim \times 20000)$. This procedure was necessary to eliminate the material which, given its density, could have been mistakenly considered as glycogen by the computer. In addition, for the subsarcolemmal images, this procedure allowed to standardise the extent to which the myofibrillar tissue was included in the analysis. To this end, it was established that the first myofibril, adjacent to the sarcolemma, and the corresponding first inter-myofibrillar space (but not the second myofibril) were always included in the analysis. The extra myofibrillar material was therefore eliminated from the analysis as described in the section "Cleaning the image". 


\section{(3)}

\section{Calibration.}

In order to ensure an optimal level of precision in all measurements, it was important to proceed with a precise calibration. Calibration procedures were applied twice in the analysis process: (a) during the electron microscope imaging and (b) during the digitising process.

The electron microscope was calibrated according to a standard calibration grid (cross-grating replica from SPI Supplies West Chester PA.; 2160 grid lines per mm). A negative of the calibration grid was first taken at $20000 \mathrm{X}$; this magnification represents the one used for all analyses. Directly from the negative, the width of a single grid line was estimated in $\mathrm{mm}$ (width of 10 grid lines was averaged). The width of a single grid line in $\mathrm{mm}$ was then multiplied by 2160 , which represents the number of grid lines per $\mathrm{mm}$ (as set by the company). This value hence represents the actual magnification of the electron microscope at the time at which the calibration grid was examined. By convention, a magnification of $20000 \mathrm{X}$ represents a magnification at which $1 \mu \mathrm{m}$ in the electron microscope represents $2 \mathrm{~cm}$ on the negative, and a magnification of $5000 \mathrm{X}$ represents a magnification at which $1 \mathrm{um}$ in the electron microscope represents $0.5 \mathrm{~cm}$ on the negative. This procedure is accomplished in order to account for day to day variation in the precision of the electron microscope. In summary:

if $\quad 1 \mathrm{~mm}=2160$ grid lines (on calibration grid);

and if 1 grid line $=8.8 \mathrm{~mm}$ (on the negatives on a given day);

then $\quad$ magnification $=2160 \times 8.8=19008 \times$ ( on that day);

therefore $\quad 1 \mu \mathrm{m}$ in the electron microscope $=1.90 \mathrm{~cm}$ on the negative (on that day).

This value was then used during the digitising process. During each digitising 
session, the distance between the camera and the negative was precisely maintained and a standard ruler was digitised in addition to the negatives. The digitised image of the ruler was then used for the calibration of all the negatives digitised during that calibration session, where $1.90 \mathrm{~cm}$ on the image was equal to $1 \mu \mathrm{m}$ on the electron microscope (as calculated above).

Single particle analysis.

As described in the previous section "Analysis of subcellular glycogen content and distribution", this analysis determined the average diameter and area of the particles that were isolated enough to be considered individually (as opposed to those clustered together). The particle volume was then calculated based on the diameter measurement as explained in the calculation section. It was therefore necessary to establish criteria for particle inclusion and exclusion for each image. These criteria were standardised and saved as an "environment" file of Image Pro Plus ( $)$. The "environment" was therefore reset every time there was a change in calibration. This latter precaution ensured that the same criteria for inclusion and exclusion were used for every single image analysed, regardless of their respective calibration. The criteria of the environments for the single particle analysis were as follows:

\begin{tabular}{|l|c|c|c|}
\hline Criteria & Units & Minimal value & Maximal value \\
\hline Area & $\mu \mathrm{m}^{2}$ & 0.00005 & 0.0012 \\
\hline Aspect & & 1 & 2 \\
\hline Density (mean) & & $\propto-$ & $\propto+$ \\
\hline Diameter & $\mu \mathrm{m}$ & 0 & $\propto+$ \\
\hline IOD & & $\propto-$ & $\propto+$ \\
\hline Roundness & & 0 & 1.2 \\
\hline
\end{tabular}


These criteria ensured that any particle that did not have an area, aspect ratio, and roundness within these ranges (see table above), was excluded from the single particle average (both diameter and area). Once the environments were standardised, a Macro was designed and applied to calculate the average particle diameter and area for the image. A copy of the Macro designed to execute this task is found in Appendix C.

Briefly, the Macro consisted of selecting the optical density threshold that would encompass all the glycogen particles, without including the background (muscle tissue). This step was critical as it allowed selection of the area of the image covered with glycogen. When post-fixed with potassium ferricyanide $\left(\mathrm{K}_{4} \mathrm{Fe}(\mathrm{CN})_{6}\right)$, glycogen can be differentiated from the background muscle tissue on the basis of its greater optical density. During the optical density threshold selection procedure (Image Pro Plus $\otimes$ function), a histogram of the optical density of all the pixels in the image being analysed is automatically produced. Based on this histogram, the point at which the frequency distribution shifts from increasing linearly to increasing exponentially represents the point at which the background starts being included in the selection. The shift from a linear to an exponential increase in frequency distribution is due to the fact that compared to the glycogen, the muscle tissue background is more homogeneous in terms of optical density, and represents a greater total area of the image. This specific shift point in the slope was the specific threshold selected for every image. This allowed standardising the optical density threshold for every image, independently of the general relative optical density of each image.

The next step consisted of using the "Edit / Draw and merge objects" function of Image Pro Plus $\otimes$ to trace around the "background noise" that was mistakenly selected by the computer as glycogen. The "background noise" represents the darkest actin, myosin, and other muscle tissue background constituents, which were introduced in the 
optical density analysis due to the imperfection in enhancing exclusively the glycogen. In addition, the particles that evidently represented a cluster of particles, as opposed to the ones isolated individually, were selected using the same function. The computer then automatically deleted these areas of disinterest, as they did not meet the inclusion criteria selected in the environment (area $>0.0012 \mu \mathrm{m}^{2}$ ). Finally, both the measurement data (including each individual particle statistic) and the statistics (including the descriptive statistics on all these individual particles), were stored in Excel. All the images from one fibre (M1 to M6 and S1 to S5) were stored in the same Excel file.

(5) Glycogen content analysis.

As described in a previous section (Analysis of subceltular glycogen content and distribution), the glycogen content analysis consisted of determining the total area of the image consisting of glycogen (glycogen area). The glycogen volume and \# of glycogen particles were then calculated (see calculation section). Criteria of inclusion and exclusion were once again necessary to prevent background noise from being considered as glycogen. This was done by adjusting the "environments" (from Image Pro Plus (c) every time there was a change in calibration. The criteria of the environments for the glycogen content analysis were as follow:

\begin{tabular}{|l|c|c|c|}
\hline Criteria & Units & Minimal value & Maximal value \\
\hline Area & $\mu \mathrm{m}^{2}$ & 0.00005 & 300 \\
\hline Density (mean) & & $\propto-$ & $\propto+$ \\
\hline IOD & & $\propto-$ & $\propto+$ \\
\hline
\end{tabular}

These criteria ensured that any particle smaller than the minimal value of $0.00005 \mathrm{um}^{2}$ (see table above) was excluded from the glycogen content analysis. This 
minimal value was determined during preliminary analysis, and consisted of the smallest observed glycogen particle area. This procedure allowed eliminating the background noise, which tends to appear as numerous particles of minimal area. Once the environments were standardised, a Macro was designed and applied to calculate the glycogen content for the image. A copy of the Macro designed to execute this task is found in Appendix C.

Briefly, the macro consisted of manually choosing the density threshold that would encompass all the glycogen particles (Image Pro Plus ® function). To standardise, the density threshold for each image was exactly the same as the one used for single particle analysis. The next step consisted of ensuring that no particle that was located on the border of the image was selected. This step was done to prevent inclusion or exclusion biases for particles that could not clearly be distinguished, due to their location on the border of the image. To do so, the "Edit / Split object" function of Image Pro Plus $\otimes$ was selected, and the investigator manually circled the inside borders of the image. Similarly to what was done in the single particle analysis, the next step consisted of using the "Edit / Draw and merge objects" function of Image Pro Plus $\otimes$ to trace around all the background noise that was mistakenly selected by the computer as glycogen. The function "Edit / Toggle objects on and off" was then used to delete those undesired areas. Finally, the function "View / Statistics" was selected to show the statistics, which were then stored in Excel adjacent to the single particles data for the same image (described previously). The glycogen selected in the above-mentioned procedure was named the "total glycogen area" for each image. For the subsarcolemmal images (S1 to S5), no further glycogen content analysis was performed. However, for all the superficial and deep myofibrillar images (M1 to M6), this total glycogen area was further subdivided into intra- and inter-myofibrillar regions. 
The distinction between intra- and inter-myofibrillar glycogen was performed immediately following the total glycogen area analysis, while the glycogen was still selected on the image. The function "Edit / Draw and merge objects" was used to frame all the glycogen that was located in the inter-myofibrillar space, that is, between two myofibrils. The function "Edit / Toggle objects on and off" was then used to delete these frames and, therefore, all the inter-myofibrillar glycogen. Hence, the remainder of the glycogen on the image was the intra-myofibrillar glycogen area. Once again, this glycogen area was stored in Excel, adjacent to the total glycogen area statistics for the same image. The inter-myofibrillar glycogen was subsequently calculated by simple subtraction (total glycogen area - intra-myofibrillar glycogen area = inter-myofibrillar glycogen area).

(6) Background analysis.

In order to account for the background optical density of each image (background muscle tissue optical density), a rapid measure was performed for every image. The background density of each image represents the optical density of the actin, myosin, and other proteins that constitute the actual skeletal muscle tissue. Most of the variation among images is caused by water content and morphological fibre type differences. However, other non-physiological variation is introduced through the electron microscope, digitising system, and other histochemical processes. A Macro was designed and applied to calculate the background optical density of each image. A copy of the Macro designed to execute this task is found in Appendix C.

Briefly, this Macro consisted of manually selecting a background area, free of glycogen or other cellular organelles, and determining its optical density. The statistics were then stored in Excel, adjacent to the other statistics for that image. 
(7) Z-line width measurements.

It was necessary to measure the average width of the Z-lines of each myofibrillar image ( $M 1$ to $M 6$ ). This measure was then used as part of the fibre typing procedure (as described in the next section: Fibre typing). This step was done with the function "Measure / Measurements" in Image Pro Plus $\otimes$. The "oblique bar" icon was selected, and the Z-line width was manually traced for as many Z-lines as possible for each image, depending on Z-line availability (between 5 and 20 per image). The average Zline width was determined for each image, and was used to determine the fibre's mean Z-line width. The fibre's mean Z-line width simply consisted of an average of the Z-line width values obtained for images M1 to M6. Figure 15 demonstrates the Z-line width measurement procedure. 


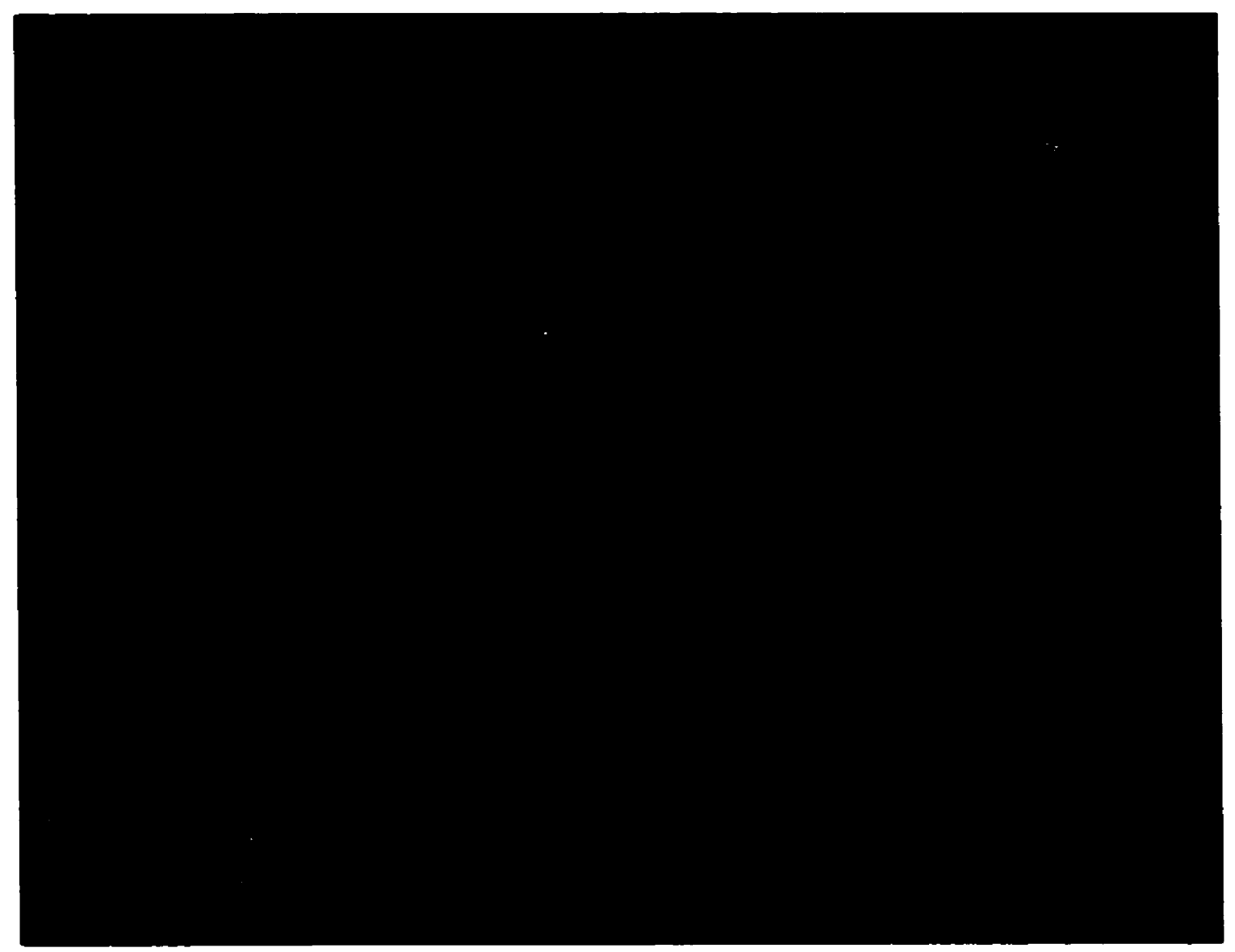

Figure 15. Illustration of the technique used to measure the Z-line width. For each image, as many $Z$ lines as possible were measured (between 5 and 20 per image) $(-X 20000)$. Only three measurements are shown here. 
(8) Image dimension measurements.

Finally, the total area of each image was measured using the same function "Measure / Measurements" in Image Pro Plus ®. For the myofibrillar images (M1 to M6), the full area of the image was calculated by tracing the border of the image. For a given fibre, all myofibrillar images had the same area (since they were all digitised and calibrated during a given session). For the subsarcolemmal images ( $\$ 1$ to $\mathrm{SE}$ ), the area of the subsarcolemmal space included in the analysis was measured. This was again performed using the same Image Pro Plus (8) function. In contrast to the myofibrillar images, the subsarcolemmal images did not all have the same area since only the glycogen in the subsarcolemmal space and the first myofibrils (including the first intermyofibrillar space) was of interest; therefore, the rest of the image was covered with white (as previously described). The glycogen areas, both for the myofibrillar and the subsarcolemmal spaces, were therefore reported as $\mu \mathrm{m}^{2}$ of glycogen $/ \mu \mathrm{m}^{2}$ of muscle tissue.

It is important to note that the calibration procedures were unconditionally performed before the steps 4 to 8 above.

In summary, the present analysis of glycogen distribution comprised, for each image, the following steps:

1. Electron microscopy of specific muscle samples to obtain negatives to be analysed.

2. Digitising of the negatives into the "TIFF" format and storage of the images on the computer.

3. Preparation of the images in order to optimise the visualisation of the glycogen.

4. Selective elimination of the undesired organelles and material that could have been falsely included as glycogen by the analysis program.

5. Calibration. 
6. Single particle analysis in order to determine mean image single particle diameter and area (volume to be calculated).

7. Glycogen content analysis in order to determine the image total, as well as intra- and inter-myofibrillar (in the case of myofibril images only) glycogen area (glycogen volume and \# of glycogen particles to be calculated).

8. Background optical density analysis.

9. Z-line width measurements.

10. Image dimension measurements.

11. Calculations (to be described in a following section).

Fibre typing.

The next part of the analysis consisted of determining the fibre type of each fibre analysed. Preliminary fibre type discrimination was conducted during the electron microscopic session. The detailed fibre type determination was performed following the subcellular glycogen distribution analysis. It has been demonstrated in mammalian skeletal muscle that Z-lines vary in thickness among fibre types (Friden et al. 1989; Landon 1992; Payne et al. 1975; Schmalbruch and Kamieniecka 1974; Sjostrom et al. 1982b). Although the values reported vary slightly among studies, it is clear that in any particular muscle, the widest Z-lines are found in the type I fibres and the narrowest in the type IIB fibres (reviewed by Landon 1992). Eisenberg (1983) concluded that in any one preparation, the upper and lower limits of the measured range of Z-line values may be expected to correspond to type I and type IIB fibres, respectively, but that other independent criteria are required to identify fibres containing Z-lines of intermediate widths. On this basis, we established a protocol to differentiate fibre types based partly on their Z-line width (Friden et al. 1989; Landon 1992; Sjostrom et al. 1982): fibres possessing a Z-line width smaller than $65 \mathrm{~nm}$ were considered type IIB; fibres 
possessing a Z-line width greater than $100 \mathrm{~nm}$ were considered type I. For the fibres with a measured Z-line width ranging between 65 and $100 \mathrm{~nm}$, three independent investigators determined the fibre type based on the following criteria from Landon (Table 1.2, p. 40, 1992): (1) lipid content; (2) shape and content of the mitochondria; (3) sarcoplasmic reticulum and T-tubule appearance. This specific fibre typing procedure was used for the majority of the fibres, since only approximately $30 \%$ of the fibres that we analysed had a Z-line width smaller than 65 or greater than $100 \mathrm{~nm}$. Using this fibre typing procedure, the means \pm S.E.M. and range $(\min -\max )$ of $Z$-line width for type 1 , IIA and IIB were $95 \pm 2(80-119 \mathrm{~nm}), 78 \pm 1(65-93 \mathrm{~nm})$, and $68 \pm 1(60-76 \mathrm{~nm})$, respectively. These values are in agreement with those reported previously in human skeletal muscles (Friden et al. 1989; Landon 1992; Payne et al. 1975; Schmalbruch and Kamieniecka 1974; Sjostrom 1982b). Furthermore, these Z-line widths have been compared with conventional histochemical (Eisenberg and Kuda 1976; Payne et al. 1975) and myofibrillar ATPase (Sjostrom 1982b) schemes of classifications, suggesting that indeed the classifications were correct. Figures 16,17 and 18 illustrate examples of the three different fibre types in the same muscle biopsy. 
Figure 16. Illustration of a typical type I fibre in a given subject. The $Z$-line $(Z)$ is wide $(110 \mathrm{~nm})$. There are numerous mitochondria $(M)$, most of which are transverse. There are consistent intermyofibrillar lipid droplets (LD). The triads (circled) are inconspicuous. 
Figure 17. Illustration of a typical type IIA fibre in the same subject as in Figure 16 . The Z-line (Z) is thinner $(90 \mathrm{~nm})$ than in a type I fibre. There are numerous mitochondria $(M)$, a significant number of which are longitudinal. The lipid droplets are sparse (none in this image). The triads (circled) are better defined compared to type I fibre. 


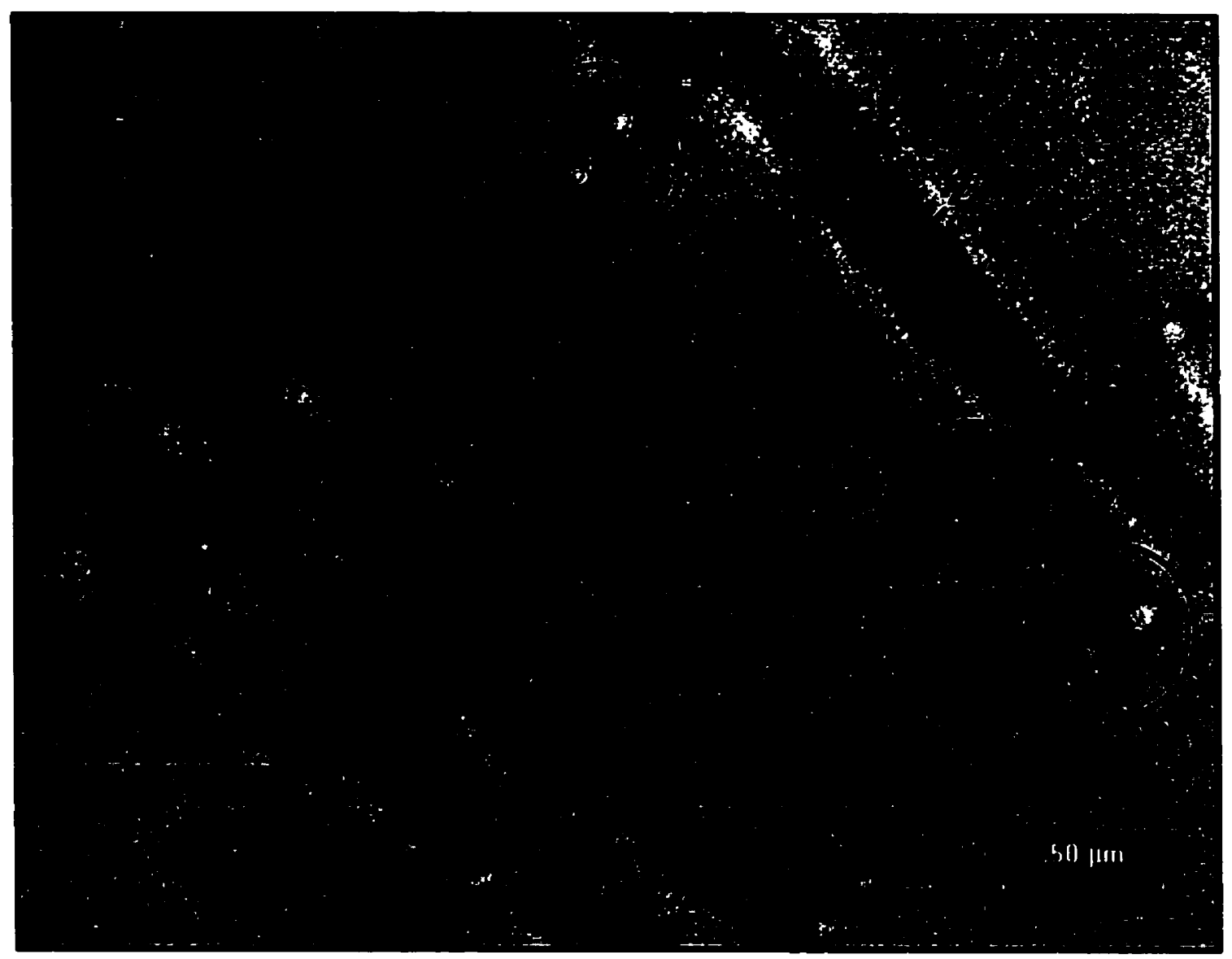

Figure 18. Illustration of a typical type IIB fibre in the same subject as in Figures 16 and 17. The Zline $(Z)$ is thinner $(70 \mathrm{~nm})$ than in type I and IIA fibres. There are very few mitochondria present (none in this image). The lipid droplets are absent. The triads (circled) are consistently well defined. 
Reliability and validity testing.

Since the present technique is novel as a process to quantify the subcellular distribution of muscle glycogen, an extensive evaluation of its reliability and validity was essential.

\section{Reliability.}

In order to test the reliability of the method, both intra- and inter-observer reliability were evaluated. The former involved the main investigator performing analysis of two subjects (i.e. images of 10 different fibres from 2 different biopsies) twice, at least two weeks apart. For the latter, an independent investigator performed the analysis of three subjects (i.e. images of 15 different fibres from 3 different biopsies), which were also analysed by the main investigator.

Intra- and inter-observer reliability were evaluated using these calculations for each dependent variable: (1) the difference, (2) the \% difference, (3) the absolute difference, (4) the \% absolute difference, (5) the square root of the mean difference squared (RMS), (6) the \% RMS, (7) the coefficient of correlation, and (8) t-test comparing the two respective groups of data. The following is a summary of the formula used to calculate the above criteria:

Difference $=X_{\text {AVG }}-Y_{\text {AVG }}$

$\%$ Difference $=\left[\left(X_{A V G}-Y_{A V G}\right) \times 100\right] /\left[\left(X_{A V G}+Y_{A V G}\right) / 2\right]$

Absolute difference $=\Sigma\left|X_{i}-Y_{i}\right| / n$

$\%$ Absolute difference $=[$ Absolute difference $\times 100] /\left[\left(X_{\text {AVG }}+Y_{\text {AVG }}\right) / 2\right]$

RMS $=\left[\Sigma\left(X_{i}-Y_{i}\right)^{2} / n\right]^{0.5}$

$\%$ RMS $=[R M S \times 100] /\left[\left(X_{\text {AvG }}+Y_{\text {AvG }}\right) / 2\right]$ 
where $X$ and $Y$ represent the two analyses being compared (either two independent investigators (inter-observer reliability) or two distinct analyses of the same investigator (intra-observer reliability)); $X_{i}$ and $Y_{i}$ represent the dependent variable values for one single image, $n$ is the number of images and $X_{A V G}$ and $Y_{A V G}$ represent the average values across all images within an analysis.

Table 3 presents the results of the intra- and inter-observer reliability testing for different variables. The results show our TEM technique of glycogen analysis to be reliable and repeatable for a given examiner. The average percentage difference between the two analyses was less than $2 \%$ for all the variables measured. In addition, the percentage of the RMS, a very conservative indicator of reliability, was less than $11 \%$ for each of the variables. Furthermore, a strong correlation, as indicated by the coefficient of variation squared $\left(r^{2}\right)$, was significant for all the variables ( $p$ values not shown) and there were no significant differences between results of the two independent analyses performed by the main investigator.

The results also demonstrate that our TEM technique for glycogen analysis was reliable and repeatable between two independent investigators. The average percentage difference between the results was less than $4 \%$ for all the variables measured. This level of variation is less than the $5-10 \%$ that has been reported (Adamo and Graham 1998a; Esbjornsson-Liljedahl et al. 1999) for repeated analysis using standard biochemical methods for measuring muscle glycogen. For all but one of the variables assessed, the $Z$-line width, the strength of the correlation was confirmed by a $r^{2}$ value greater than 0.95 . For this variable, although the correlation was strong $\left(r^{2}=\right.$ $0.49)$ and significant $(p<0.0001)$, the means were significantly different. This difference is probably related to the very low variance associated with the measurement of the Zline, compared to the other variables. It is important to note that the average absolute 
difference between the investigators was only $2.7 \mathrm{~nm}$. Such a small magnitude of difference is not critical as Z-line width was only employed for fibre type classification, and such a minimal difference is not likely to affect our classification (see fibre typing section above).

Table 3. Intra- and inter-observer reliability testing.

\begin{tabular}{|c|c|c|c|c|c|c|c|c|c|c|c|}
\hline \multicolumn{12}{|l|}{ Intra-rater reliability } \\
\hline Variables & images & tst maan & 2nd maan & $\Delta$ & $\% \Delta$ & Absolute $\Delta$ & $\%$ absolute $\Delta$ & Rus & \% RuS & $r^{2}$ & t-test \\
\hline Z-line (nm) & 46 & 71.4 & 70.5 & 0.9 & 1.3 & 2.5 & 3.6 & 3.3 & 4.7 & 0.79 & $p=0.53$ \\
\hline Glycogen area $\left(\mathrm{nm}^{2} \times 10^{3}\right)$ & 34 & 62.83 & 62.57 & 0.08 & 0.1 & 3.27 & 5.2 & 4.9 & 7.9 & 0.98 & $p=0.99$ \\
\hline Single particle area $\left(\mathrm{nm}^{2}\right)$ & 52 & 0.353 & 0.346 & 0.007 & 2.0 & 0.018 & 5.2 & 0.023 & 6.6 & 0.98 & $p=0.80$ \\
\hline \multicolumn{12}{|l|}{ Inter-rater rellability } \\
\hline Variables & * Images & Ist mean & 2nd mean & $\Delta$ & $\% \Delta$ & Absolute $\Delta$ & $\%$ Absolute 4 & Rus & \% RMS & $r^{2}$ & $t-\tan t$ \\
\hline Z-line (nm) & 95 & 810 & 78.3 & 2.7 & 3.4 & 5.8 & 7.3 & 7.3 & 9.2 & 0.49 & $p=0.04$ \\
\hline Glycogen area $\left(n \mathrm{~m}^{2} \times 10^{3}\right)$ & 72 & 71.57 & 72.20 & 0.6 & .1 .0 & 4.69 & 6.5 & 7.7 & 10.7 & 0.97 & $p=0.93$ \\
\hline Single particle area $\left(\mathrm{nm}^{2}\right)$ & 109 & 0.354 & 0.355 & .0 .001 & .0 .2 & 0.023 & 6.5 & 0.034 & 9.6 & 0.95 & $p=0.97$ \\
\hline
\end{tabular}

"\# of images" is the number of images which were analysed by each of the investigators (inter-observer reliability) or by the same investigator at two different points in time (intra-observer reliability), for that particular variable. " $r^{2 n}$ is the squared coefficient of correlation between the two sets of images to be compared. "t-test" is the $p$ value of the t-test comparing the two sets of images. See text for more details.

Furthermore, it was necessary to ensure that the intra- and inter-myofibrillar particles did not differ in terms of their average single particle variables (diameter, area and volume). This was imperative since the single particle analysis did not differentiate between the intra-and inter-myofibrillar particles and, therefore, could not detect such a difference between the intra- and inter-myofibrillar particle size. Therefore, before 
starting our single particle analysis, it was necessary to ensure that the size of the single particles did not differ between these locations. Standard photographs and calibration grids were taken from three different samples (a subject with low muscle glycogen, a subject with high muscle glycogen, and a McArdle's disease (Glycogen storage disease $V$ ) patient) at a magnification of $40000 \mathrm{X}$. An independent, blinded investigator manually outlined and traced individual glycogen particles in each of the intra- and intermyofibrillar spaces of different images from these samples. The MOP Videoplan program (Kontron, Germany) was precisely calibrated and used in this "manual" determination of the diameter of individual glycogen particles. A direct comparison was then performed between the intra- and inter-myofibrillar particle diameters. Since the majority of the inter-myofibrillar granules are arranged in clusters and the majority of the intra-myofibrillar granules are isolated from each other (Figure 9), this analysis also allowed elimination of the possibility of a difference between the size of particles arranged in clusters compared to those isolated. The results of this preliminary analysis for three different samples and more than 800 single particles are shown in Figure 19.

The analysis revealed no difference between the diameter of the intra- and intermyofibrillar particles. In fact, the average diameter difference between intra- and intermyofibrillar particle particles was found to be less than $0.5 \mathrm{~nm}(p=0.25)$. Thus, the data from the intra- and inter-myofibrillar space were combined during the single particle analyses. As mentioned above, these results also suggest that the dimensions of granules are not different if they are in clusters. 


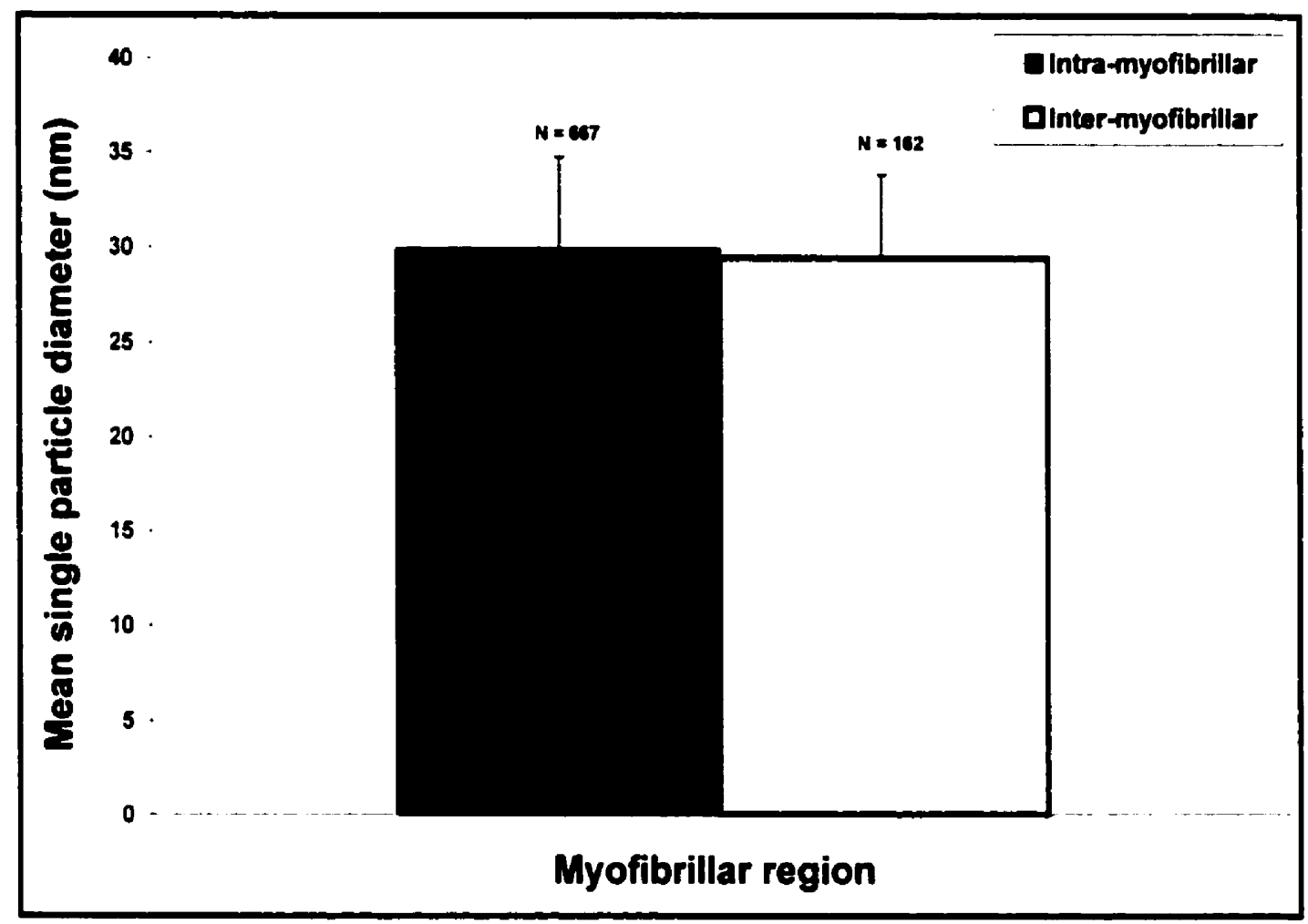

Figure 19. Illustration of the results from the preliminary analysis manually comparing the diameter of the intra- and inter-myofibrillar regions. Details are provided in the text.

Finally, a comparison was performed between cells of the same fibre type within a given sample, to determine the variation in glycogen concentration between two type I or two type IIA fibres from the same subject. To do so, the coefficients of variation were calculated for each pair of glycogen values (i.e. two type I fibres from subject 1 , subject 2 , subject 3 , etc). The average of these was used as a representation of the variation in glycogen concentration within one fibre type. Given the low prevalence of type IIB fibres, they were not included in this calculation. The results showed that the averaged coefficients of variation between two type I fibres and two type IIA fibres from the same muscle biopsy were 11 and $14 \%$, respectively. These values are comparable to the 9 
$\%$ coefficient of variation reported for biochemically determined glycogen values for two different parts of the same muscle fibre (Essen and Henriksson 1974). Furthermore, our results agree with those of White and Snow (1987), who found the coefficient of variation for a histochemical technique (PAS) to be $8 \%$ upon examination of similar fibres from serial sections of the same muscle.

Validity.

In order to test the validity of our technique, we performed regression analysis between the glycogen concentration obtained by the standard biochemical measurements and that obtained by our TEM-based technique. The samples ranged between glycogen concentrations of 13 and $515 \mathrm{mmol}$ of glucosyl units / $\mathrm{kg} \mathrm{dw}$, as determined biochemically. Figures 20 and 21 depict the results of the regression analysis performed between the biochemically determined muscle glycogen concentration, and the TEM-based myofibrillar glycogen volume (Figure 20) and single glycogen particle volume (Figure 21). The strong significant correlation between those variables suggests that the TEM-based measure of glycogen is valid in assessing total muscle glycogen content. Furthermore, the subsarcolemmal glycogen volume was also found to correlate with the biochemically determined muscle glycogen concentration $\left(r^{2}=\right.$ $0.85, p<0.00001$ ) (data not shown). In addition, the TEM-based myofibrillar glycogen concentration was found to be correlated with the biochemically determined $P G\left(r^{2}=\right.$ $0.80, p<0.00001)$ and $M G\left(r^{2}=0.85, p<0.00001\right.$ ) (data not shown). Taken together, the results of these regression analyses support the validity of our technique in evaluating the subcellular human skeletal muscle glycogen distribution. 


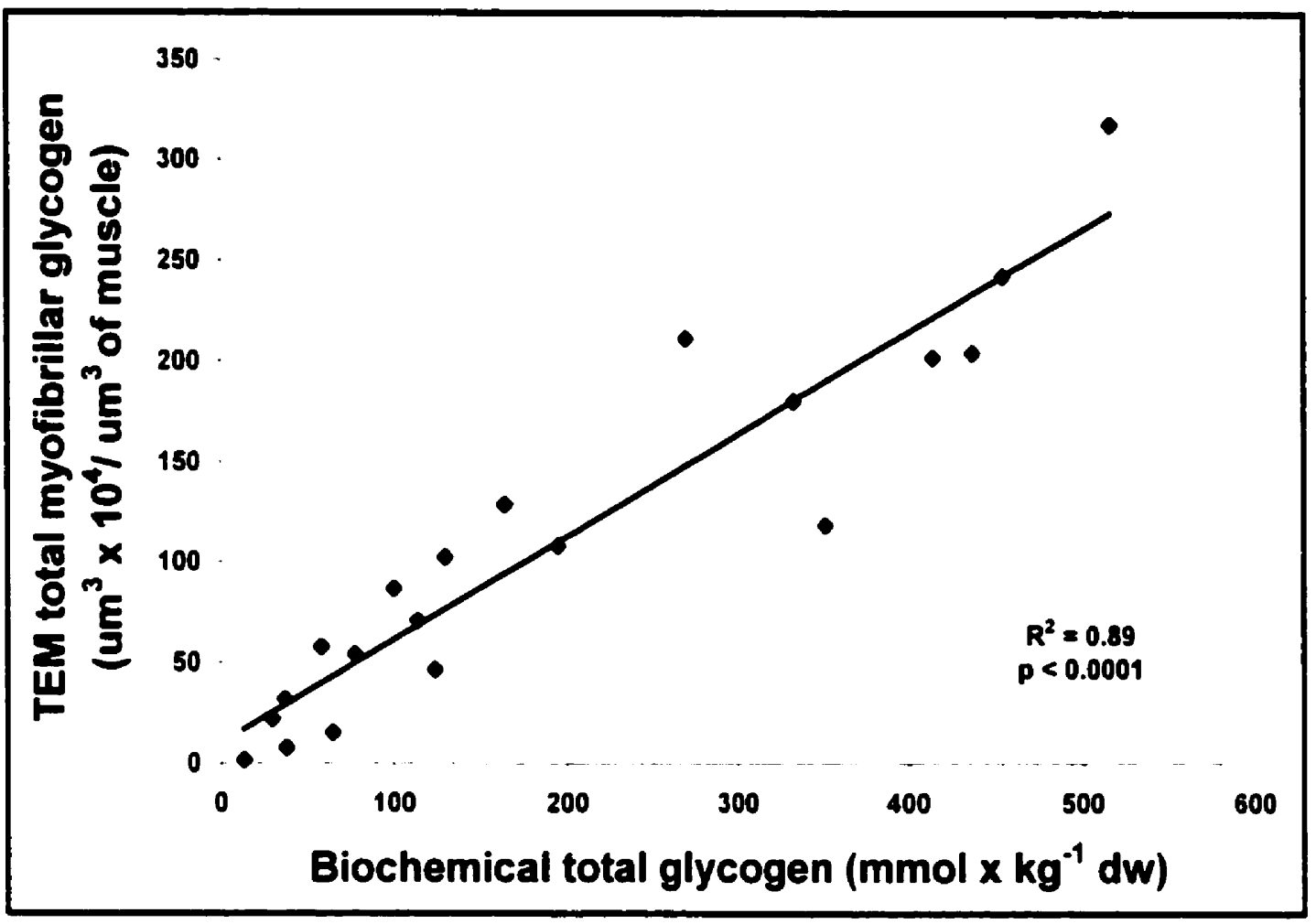

Figure 20. Relation between the biochemically determined total muscle glycogen concentration ( $\mathrm{mmol}$

- $\left.\mathrm{kg} \mathrm{dm}^{-1}\right)$ and the TEM based myofibrillar glycogen volume $\left(\mu \mathrm{m}^{3} \times 10^{4} / \mu \mathrm{m}^{3}\right.$ of muscle) for 20 biopsy samples. The TEM-based glycogen volume was weighted for muscle fibre type proportions (described in a previous section). There was a strong positive correlation between the traditionally measured total glycogen concentration (biochemically determined), and our TEM-based glycogen variables $\left(r^{2}=0.89\right.$; $p=0.0001$ ). 


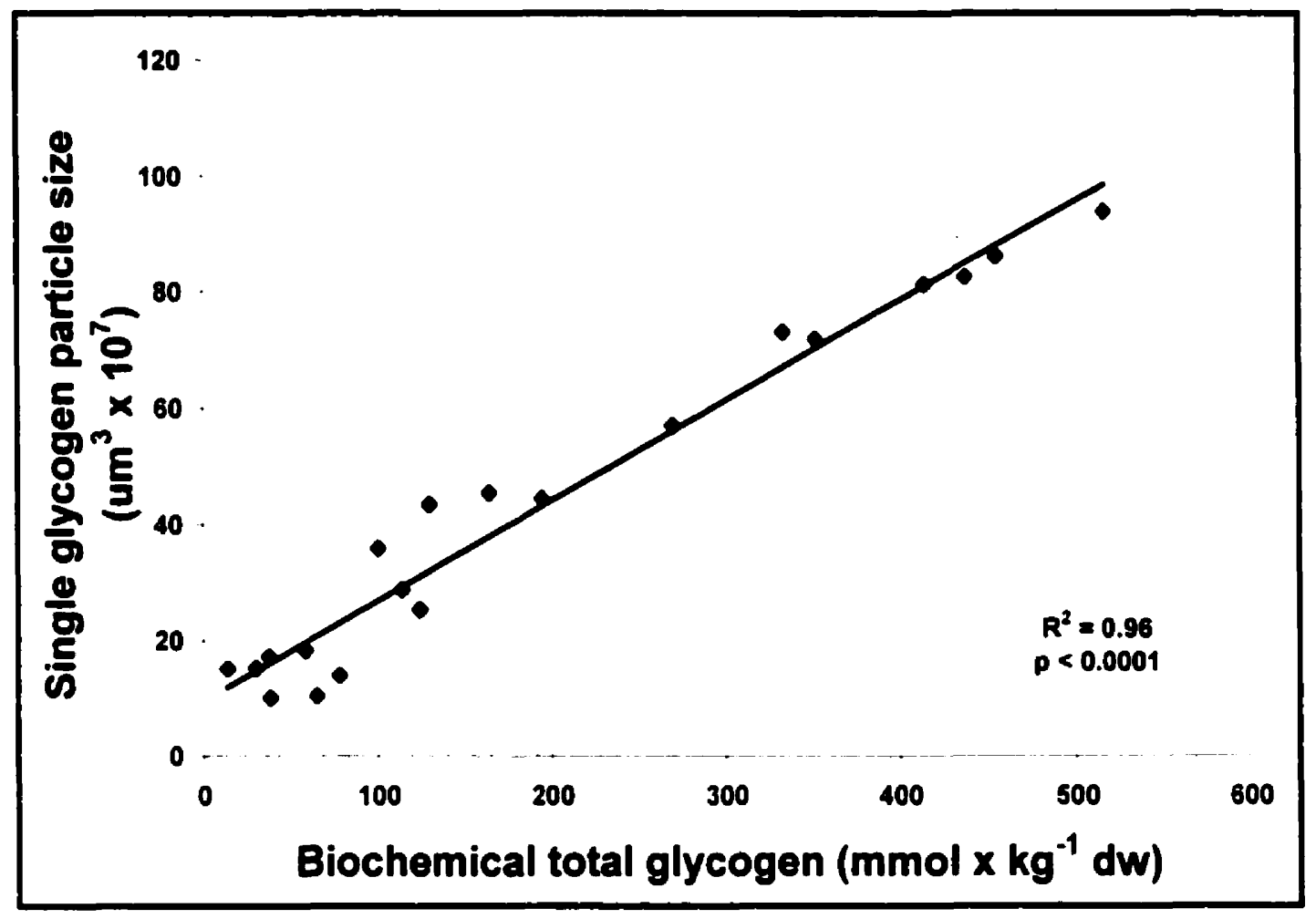

Figure 21. Relation between the biochemically determined total muscle glycogen concentration (mmol - $\left.\mathrm{kg} \mathrm{dm}^{-1}\right)$ and the TEM-based single glycogen particle volume $\left(\mu \mathrm{m}^{3} \times 10^{7}\right)$ for 5 subjects. There was a strong positive correlation between the traditionally measured total glycogen concentration (biochemically determined), and our TEM-based glycogen variables $\left(r^{2}=0.96 ; p=0.0001\right)$.

Taken together, the results of this extensive analysis demonstrate that our novel TEM-based technique for quantifying the subcellular distribution of human skeletal muscle glycogen is valid and reliable, both within and between investigators. 


\section{Calculations.}

Biochemical analysis.

As mentioned previously, an independent investigator performed the biochemical analyses. Total glycogen (Gt) was calculated as the sum of PG and MG (Adamo 1998a). The percentage of $\mathrm{Gt}$ in the form of PG (\% PG) was determined by dividing the concentration of PG by the Gt concentration and multiplying by 100 (\% PG = PG / Gt $\times 100$ ). The main investigator of the histochemical analyses was blinded to the results of the biochemical analyses.

TEM analysis.

Dependent variables. For each muscle fibre analysed using TEM, the following dependent variables were considered:

(1) mean single particle diameter $(\mathrm{nm})$ for deep myofibrillar space (Figure 3: average of images M2, M4, and M6), superficial myofibrillar space (Figure 3: average of images M1, M3, and M5), subsarcolemmal space (Figure 3: average of images S1-S5), and total fibre (images from all three spaces; Figure 3: average of images S1-S5 and M1M6);

(2) mean single particle volume $\left(\mathrm{nm}^{3}\right)$ for deep myofibrillar space, superficial myofibrillar space, subsarcolemmal space, and total fibre;

(3) number of glycogen particles (per $\mu \mathrm{m}^{3}$ of muscle tissue) for deep myofibrillar space, superficial myofibrillar space, subsarcolemmal space, and total myofibrillar space (average of the pooled deep and superficial myofibrillar space images; Figure 3: images $M 1-M 6)$; 
(4) glycogen volume $\left(\mu \mathrm{m}^{3} / \mu \mathrm{m}^{3}\right.$ of muscle tissue) for deep myofibrillar space, superficial myofibrillar space, subsarcolemmal space, and total myofibrillar space.

While the single particle and total glycogen area were measured, they were only used in the calculations of the dependent variables mentioned above. Therefore, they are not listed above as dependent variables considered.

Ideally, data for the total fibre would be determined for each variable, but this could only be calculated for the single particle variables (single particle diameter and volume). The inability to obtain such a value for the number of glycogen particles and glycogen volume variables, which are both expressed per $\mu \mathrm{m}^{3}$ of muscle tissue, was due to our inability to determine the relative proportion of muscle fibre as myofibrillar and subsarcolemmal spaces. Based on our image selection protocol, it was not possible to measure the individual muscle fibre circumference and volume, which is essential in calculating the relative contribution of the subsarcolemmal space to the total fibre volume. Since the diameter of individual muscle fibres is known to range from 10 to 100 $\mu \mathrm{m}$ (Landon 1992), the error introduced by an average value would be tremendous. Hence, the variables from each fraction (subsarcolemmal vs myofibrillar) were expressed separately (except the single particle variables since they are not expressed per $\mu \mathrm{m}^{3}$ of muscle tissue), and total myofibrillar values alone were used as a representation of the fibre values.

The single particle diameter was obtained by direct measurement of the granules on the digitised images. The single particle volume was calculated, assuming that the glycogen granules are spheres, as follows: 


\section{Single particle volume $=4 \pi r^{3} / 3$, where $\pi$ is pi (3.141593) and $r^{3}$ is (diameter $\left./ 2\right)^{3}$.}

The total glycogen area was divided by the mean single particle area (both values obtained by direct measurement from the digitised images), and then by $0.070 \mu$ $m$ (thickness of a thin section) to obtain the number of glycogen particles (per $\mu \mathrm{m}^{3}$ of muscle tissue) (Williams 1977). It is important to note that the mean single particle area value used varied as a function of the subcellular location. For example, in a given fibre, if the number of glycogen particles is being calculated for a superficial myofibrillar image (Figure 3: either of M1, M3 or M5), it is the fibre's mean single particle area value for that subcellular location that is used (Figure 3: average of images M1, M3, and M5).

The glycogen volume was calculated by multiplying the number of glycogen particles (per $\mu \mathrm{m}^{3}$ of muscle tissue) by the mean single particle volume $\left(\mu \mathrm{m}^{3}\right.$ ), to obtain the glycogen volume in $\mu \mathrm{m}^{3} / \mu \mathrm{m}^{3}$ of muscle tissue.

For each fibre, the relative proportion of the myofibrillar (myofib) glycogen located in the intra-myofibrillar region (Intra / myofib), expressed as a percentage, was calculated by dividing the average intra-myofibrillar glycogen volume by the average myofibrillar glycogen volume. This value was compared between time (except in the first experiment where time was not included as a variable), fibre types (I, IIA, and IIB) and subcellular locations (deep and superficial myofibrils).

Finally, for each fibre, the ratio of the subsarcolemmal to total myofibrillar (Tmyofib) glycogen volume (Subsarc / Tmyofib), was calculated. This value was 
compared among times (except in the first experiment where time was not included as a variable) and fibre types (I, IIA, and IIB).

Average value for a muscle biopsy (weighted mean). Human skeletal muscles are composed of different fibre types, which can have different amounts of glycogen (Conlee et al. 1978; Derave et al. 2000; Fell et al. 1980; Hansen 1999; Richter et al. 1985; Vandenberghe et al. 1999). Furthermore, it is well known that human vastus lateralis muscle is not composed equally of fibres of all three types. Therefore, in order to obtain the best overall estimate for each dependent variable measured for each of our muscie samples, it was necessary to calculate a weighted average of the different fibre types for each sample. The weighted average was calculated based on the average fibre type composition of the human vastus lateralis ( $-50 \%$ type I, $\sim 40 \%$ type IIA, and $\sim 10 \%$ type IIB) reported in the literature (Barstow et al. 1996; Bouchard et al. 1986; Simoneau et al. 1995). For each muscle sample, all type I, type IIA, or type IIB fibres were averaged, to obtain one value per dependent variable per fibre type per sample. The overall weighted muscle value was then calculated according to the following formula:

\section{Weighted mean $=($ type I value $\times 0.5)+($ type $\|$ A value $\times 0.4)+($ type $\| B$ value $\times 0.1)$}

Due to the limited quantity of tissue contained in each muscle biopsy sample, the three fibre types were not necessarily present in every group of 5-6 fibres selected for each of the muscle samples. Since the weighted average required at least one fibre from each of the three fibre types, it was necessary to statistically generate values for the missing fibre types for each sample (mainly type IIB due to their low natural frequency of occurrence). The technique used to generate missing data was adapted from an equation from Steel and Torrie (1960). Briefly, a matrix was constructed to 
estimate the values of the missing fibre types. A different matrix was constructed for each time point (except in the first study where time was not included as a variable). For a given time point, the matrix included the three different fibre types on the vertical axis, and each muscle sample (one subject at the given time) on the horizontal axis. For that given time point, the matrix therefore included " $X$ " number of values, where " $X$ " corresponded to the number of fibre types (3) times the number of subjects (variable according to the study). An example of a matrix and the corresponding calculation is found in Appendix D.

In experimental designs that included many time points (studies 2 and 3), the procedure had to be repeated for each time point. Furthermore, for each time point, the procedure had to be repeated for each of the dependent variables (see previous section). These estimated values were then used to determine the overall weighted average for each muscle sample as described earlier.

A great advantage of this technique is that it allowed generating a missing value considering both subjects and fibre types variations. It is worth mentioning that, due to the minimal relative proportion of type IIB fibres in a normal human vastus lateralis muscle (Barstow et al. 1996; Bouchard et al. 1986; Simoneau et al. 1995), a large percentage of the muscle samples selected lacked that particular fibre type. It was therefore necessary to generate the missing values for approximately $75 \%$ of the muscle biopsy samples analysed. Although this percentage may appear relatively high, it is important to consider that the type $\| B$ fibres contribute to only $10 \%$ of the normal total muscle fibre type composition (Barstow et al. 1996; Bouchard et al. 1986; Simoneau et al. 1995), and therefore, to only $10 \%$ of the weighted muscle average discussed previously. 
Experimental design.

The present method was designed to quantify the intra-cellular distribution of human skeletal muscle glycogen. Figure 22 illustrates a summary of the experimental design used in the three experiments to be described in Chapters V, VI and VII.

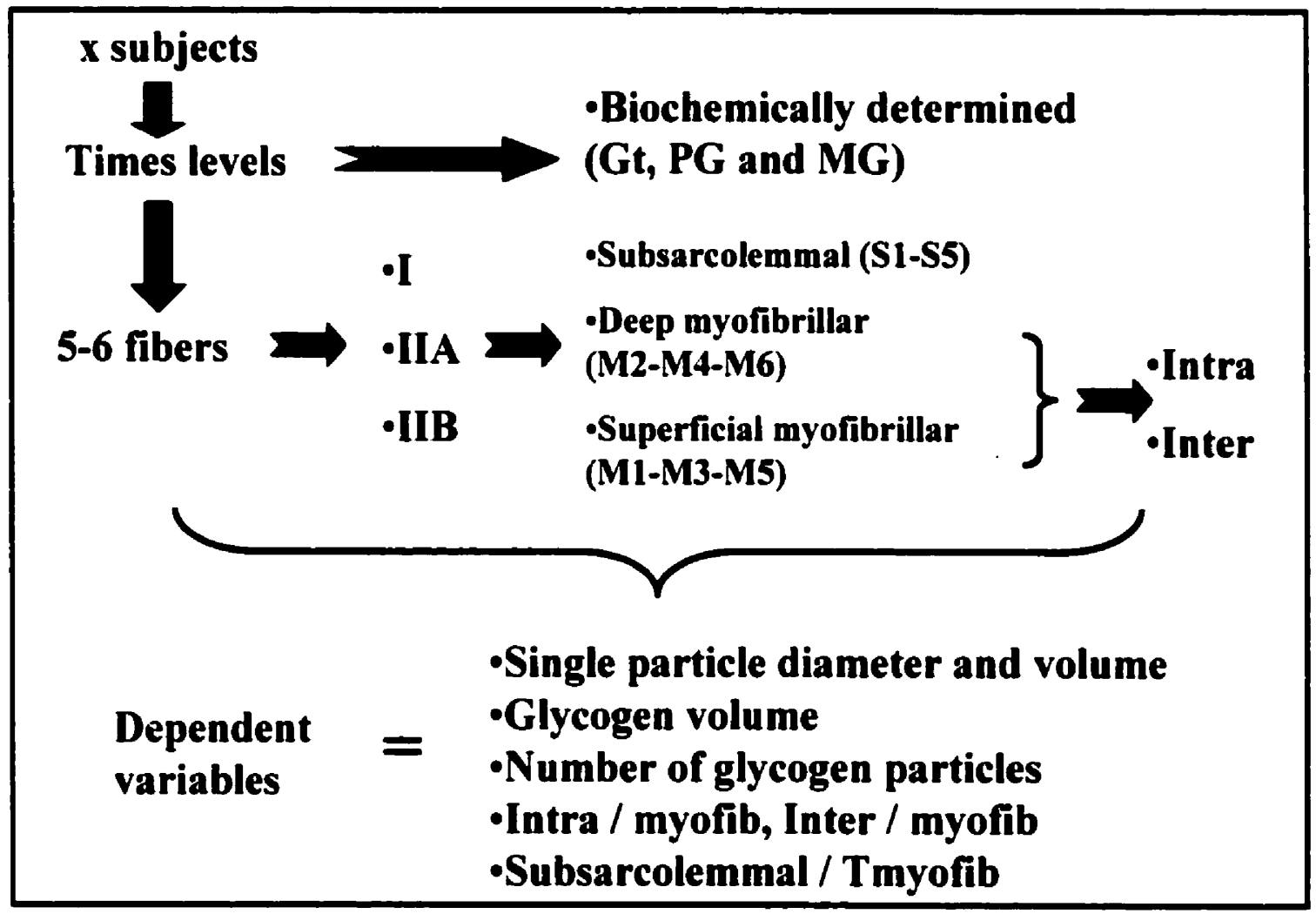

Figure 22. Summary of the experimental design. "Gt", "PG" and "MG" are the total, pro- and macroglycogen, respectively, as determined biochemically. "Intra" is intra-myofibrillar. "Inter" is intermyofibrillar. "Intra / myofib" and "Inter / myofib" is the relative proportion of myofibrillar glycogen located in the intra- and inter-myofibrillar regions, respectively. "Subsarcolemmal / Tmyofib" is the ratio of subsarcolemmal to total myofibrillar (pooled superficial and deep) glycogen. 
In summary:

A muscle biopsy at each of the time periods (except first study) was taken from each subject, and 5-6 fibres per biopsy were analysed. For each fibre, the type was determined (I, IIA, or IIB). The fibre was subdivided into subcellular locations (subsarcolemmal space, deep and superficial myofibrils). The deep and superficial myofibrillar images were further subdivided into inter- and intra-myofibrillar regions.

For each fibre, the following variables were considered:

- mean single particle diameter $(\mathrm{nm})$ for deep myofibrillar space, superficial myofibrillar space, subsarcolemmal space, and total fibre;

- mean single particle volume $\left(\mathrm{nm}^{3}\right)$ for deep myofibrillar space, superficial myofibrillar space, subsarcolemmal space, and total fibre;

- number of glycogen particles (per $\mu \mathrm{m}^{3}$ of muscle tissue) for deep myofibrillar space, superficial myofibrillar space, subsarcolemmal space, and total myofibrillar space;

- glycogen volume $\left(\mu \mathrm{m}^{3} / \mu \mathrm{m}^{3}\right.$ of muscle tissue) for deep myofibrillar space, superficial myofibrillar space, subsarcolemmal space, and total myofibrillar space;

- \% of myofibrillar glycogen volume as intra-myofibrillar glycogen (Intra / myofib);

- Ratio of subsarcolemmal to total myofibrillar glycogen volume (Subsarc / Tmyofib).

For each subject and at each of the time points (except first study), an overall weighted muscle value was calculated (as described above) for all individual dependent variables considered. 


\section{Statistics.}

Descriptive statistics were performed using Microsoft (8) Excel 1997. T-tests were used to compare intra- and inter-observer reliability. Inferential statistics were performed using the SAS \& system (Release 6.12). The multivariate repeated measures design was assessed using a mixed linear model with "Subject and Subject $x$ Time" as the random effects and "Time, Fibre types, Subcellular locations, Myofibrillar regions, and their interactions" as fixed-effects (see below for more details). When these analyses revealed significant main effects or interactions, a Tukey's studentized range procedure was used to locate the pair-wise differences. Significance was considered at $P<0.05$.

The mixed linear procedure of the SAS program (named Mixed Procedures) is a particular form of analysis of variance designed for statistical analysis of multivariate repeated measure designs. It functions by fitting a variety of mixed linear models to the data and enables us to use these fitted models to make statistical inferences about the data. In standard linear models, which are used during most simple analyses of variance (ANOVA), it is simply assumed that any score can be represented by a linear model. A mixed linear model is therefore a generalisation of a standard linear model, the generalisation being that the data are permitted to exhibit correlations (among the different variables) and non-constant variability. The primary assumptions underlying the analysis of variance performed with this mixed linear model are:

1. The data are normally distributed.

2. The means (expected values) are linear in terms of a certain set of parameters (so they can fit one or many of the proposed models).

3. The variances and covariances of the data are in terms of a different set of parameters, and they exhibit a structure matching one of those available in the Mixed Procedures. 
These can be compared to the assumptions required for an ANOVA using a standard linear models, which are:

1. The data are normally distributed.

2. The variances of observations are equal across groups (homogeneity of variance).

3. The observations are statistically independent within the group to which they belong.

4. In case of repeated measures, there must be an homogenous covariance among observations obtained from the same subject.

Although the first assumption of each model is the same (normal distribution), the other assumptions greatly differ. The mixed linear model, therefore, provides the flexibility of modelling not only the means of the data (as is the case in ANOVA using a standard linear model), but also their variances and covariances as well.

Since the normally distributed data can be modelled entirely in terms of their means and variances / covariances, the two sets of parameters in a mixed linear model actually specify the complete probability distribution of the data. The parameters of the model are referred to as "fixed-effects parameters", and the parameters of the variancecovariance model are referred to as "covariance parameters. The covariance parameters are what distinguish the mixed linear model from the standard linear model. These covariance parameters were necessary in the case of our repeated measures experimental design for two reasons. First, since the repeated measurements were taken from the same subject, these repeated measurements could be correlated or exhibit variability that changes. Second and most importantly, the experimental units on which the data are measured can be grouped into clusters (i.e. fibre types), and the data from a common cluster could be correlated. Together, these would have violated the 
three last assumptions (see above) of an ANOVA using a standard linear model.

Appendix E presents detailed instructions for SAS and explanation of the statistical output.

Finally, the data organisation for our experimental design was extremely complex since there were a number of variables involved. Appendix $F$ presents a summary of the data sheet used for analysis, as well as the parameters, variables, and effects of interest in each case. 


\section{Chapter V}

Experiment 1: Quantification of subcellular glycogen in resting human skeletal muscle: granule size, number and location. 


\section{Introduction}

Since the discovery of glycogen by Claude Bernard (Bernard 1957); numerous investigators have addressed many aspects of its metabolism. While muscle glycogen concentration has been routinely quantified biochemically, the subcellular organisation of glycogen particles has been studied much less frequently, and only with qualitative, descriptive transmission electron microscopy (TEM) methods. Wanson and Drochmans (1968) performed the first comprehensive description of rabbit skeletal muscle glycogen in its particulate $\beta$ form. Drochmans (1962) had previously examined negatively-stained liver glycogen using TEM and described three glycogen structures in liver, the $\alpha, \beta$, and $\gamma$ particles. The $\alpha$ particles were the typical liver rosettes and the $\beta$ particles were the 20-30 nm spheroid units forming the $\alpha$ rosettes. The $y$ particles were identified as $3 \mathrm{~nm}$ subunits of both $\alpha$ and $\beta$ structures. The single $\beta$ particles described in muscles by Wanson and Drochmans 1968) corresponded in size and shape to the $\beta$ subunits that constituted the $\alpha$ rosettes in liver.

Scott and Still (1968) proposed that particulate glycogen was not a molecule in the traditional static sense, but rather a dynamic organelle. In 1970, Meyer and collaborators (1970) were among the first to suggest that glycogen was complexed with proteins and represented a structural and functional unit of the muscle cells. Using TEM, they estimated the diameter of this glycogen particle to be $20-30 \mathrm{~nm}$. This value was in agreement with the average diameter $(27 \mathrm{~nm})$ of $\beta$-glycogen particles previously described by Wanson and Drochmans (1968) in intact muscles. It is now known that the granule is physically associated with a number of proteins including glycogenin, glycogen synthase, glycogen phosphorylase, phosphorylase kinase and phosphatase as well as scaffolding proteins (reviewed by Roach et al. 1998). This raises the distinct possibility that granules can be regulated regionally or even individually. 
The term $\beta$-particle has been applied to this protein-glycogen complex, and has been consistently described as a 20-30 nm particle (Rybicka 1996). Several investigators have found the $\beta$-particles to be located mainly in the subsarcolemmal and the inter-myofibrillar space, in close proximity to the sarcoplasmic reticulum (SR) (Friden et al. 1985 and 1989; Rybicka 1996; Schmalbruch and Kamieniecka 1974; Sjostrom et al. 1982). Rybicka (1996) reviewed the area and clearly supported the concept that the structures commonly interpreted as particles of glycogen actually represent dynamic organelles (glycosomes), which act as independent metabolic units with specialised functions. A heterogeneous distribution of glycosomes could result in metabolic compartments, with distinct characteristics and functions. For example, biochemical and morphological evidence has demonstrated the association of some glycosomes with the SR in both cardiac and skeletal muscles (Goldstein et al. 1985; Meyer et al. 1970; Polishchuk et al. 1995; Wanson and Drochmans 1968). This subcellular location may represent a metabolic compartment dedicated to SR function.

Ekblom and co-workers (Friden et al. 1985 and 1989; Sjostrom 1982) have provided most of our understanding of the compartmental distribution of skeletal muscle glycogen in humans. They used TEM to describe qualitatively the distribution of skeletal muscle glycogen in different subcellular locations, different muscle fibre types, and under different exercise conditions. They described glycogen as being stored in five topographically different locations (Friden et al. 1989). They also reported that exercise of different intensities metabolised glycogen from specific subcellular locations and muscle fibre types, and proposed a selective pattern of subcellular glycogen metabolism, depending on the exercise characteristics (Friden et al. 1985). Furthermore, they described two separate and distinct populations of glycogen particles with diameters of 22 and $56 \mathrm{~nm}$. They speculated that these populations might represent the acid-soluble and insoluble forms of glycogen previously described by 
Jansson (1981) (now referred to as macro- and proglycogen, respectively (Adamo et al. 1998b; Roach et al. 1998). While the results of these early studies are interesting, the methodological approach used in estimating muscle glycogen concentration lacked the objectivity and detailed information of quantitative procedures. For example, their selection of fibres and subcellular locations was not systematic, and the sample sizes were restricted owing to the labour-intensive nature of TEM work and the lack of advanced computerised processes to assist in data collection and analysis.

The purposes of the present study were 1) to develop and validate a TEM method to quantify the subcellular distribution of muscle glycogen and 2) to appiy this TEM technique to biopsy samples of resting human muscle in order to quantify the distribution of glycogen in different subcellular locations and muscle fibre types.

\section{Methods}

Subjects. The study was approved by the University of Guelph's Human Ethics Committee and conformed to the standards set by the Declaration of Helsinki. Eleven male subjects were informed in writing about the nature of the study, volunteered to participate, and signed a consent form. The mean ( \pm S.E.M.) age, height, weight, and $\mathrm{VO}_{2} \mathrm{max}$ of the subjects were $25 \pm 1 \mathrm{yr}, 184 \pm 2 \mathrm{~cm}, 86 \pm 2 \mathrm{~kg}$, and $53 \pm 2 \mathrm{~mL} \cdot \mathrm{kg}^{-1} \cdot$ $\min ^{-1}$, respectively.

Procedures. Resting muscle biopsies were obtained from the Vastus lateralis of the subjects by use of the percutaneous needle biopsy technique (Hultman 1967) with suction modification (Evans 1982). Each muscle sample was divided in two portions, for biochemical and TEM analyses. Two independent investigators carried out the biochemical and the TEM analyses. 
Analyses, calculations and statistics.

Please refer to Chapter IV - Detailed Methods.

\section{Results}

Biochemical data. The subjects had an average ( \pm S.E.M.) total muscle glycogen concentration of $380 \pm 41 \mathrm{mmol} \cdot \mathrm{kg} \mathrm{dw}^{-1}$ and $74 \pm 2 \%$ was in the proglycogen form.

Dependent variables. Figure 23 and Table 4 summarise the results for single particle analysis. There was a main effect of both fibre type $(p=0.04)$ and subcellular location $(p=0.0001)$. Post hoc analyses showed that the average particle volume was $21 \%$ greater in type IIA than in type I fibres $(p<0.05)$. Neither the $37 \%$ difference between type IIB and I, nor the $13 \%$ difference between type $\| \mathrm{B}$ and IIA fibres was significant (Table 4). In addition, the average particle volume increased with depth in the fibre (Figure 23). Post hoc analysis revealed the subsarcolemmal particle volume $(7376 \pm 48$ $\left.\eta \mathrm{m}^{3}\right)$ was smaller than the superficial $\left(8462 \pm 55 \mathrm{\eta m}^{3}\right)$ and deep $\left(8802 \pm 57 \mathrm{~nm}^{3}\right)$ myofibrillar particle volumes (both $p=0.0001$ ).

Figure 24 (A) illustrates the distribution frequency for the single particle diameter for all fibres of the 11 subjects ( $n>55000$ particles). Particle size appears to be normally distributed, ranging from 10 to $44 \mathrm{~nm}$, with an average size of approximately 25 $\mathrm{nm}$. Clearly, there is no indication of two distinct groups of particle sizes. Figure 24 (B) illustrates the relative frequency distribution (\%) for single particle diameter, within each fibre type. In agreement with the single particle volume data, the single particle diameter was significantly different between fibre types $(p=0.04)$ : $\|B(26.1 \pm 1.6 \mathrm{~nm})>\| A(25.3$ $\pm 0.5 \mathrm{~nm})>$ I $(24.5 \pm 0.9 \mathrm{~nm})$. Post-hoc analysis revealed a significant difference only between type I and type liA fibres $(p<0.05)$. 


\section{Table 4. Dependent variables.}

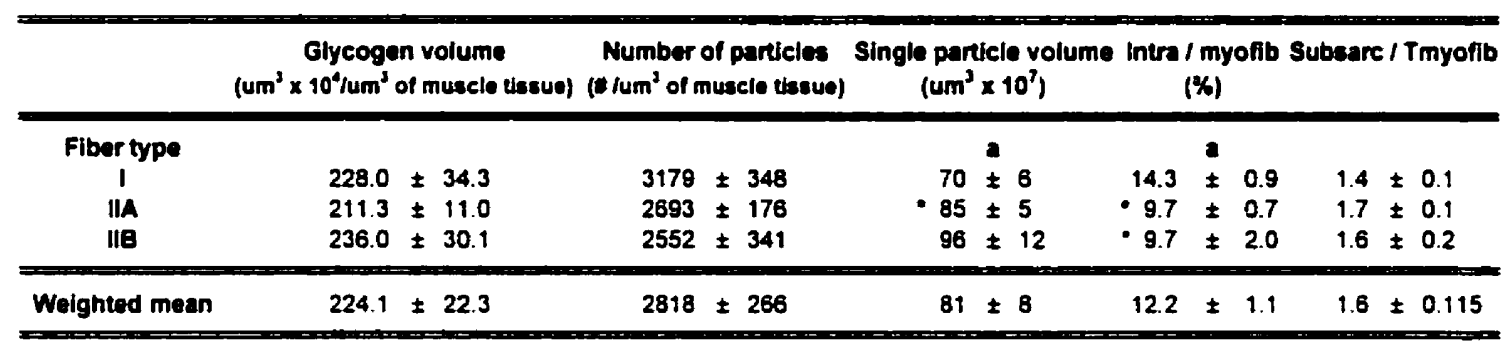

Values are mean \pm S.E.M. for the eleven subjects. "a" indicates significant main effect of fibre types (p $<0.05)$. " indicates significant difference from type I fibres $(p<0.05)$. Intra / myofib is the relative proportion of myofibrillar glycogen volume located in the intra-myofibrillar region. Subsarc / Tmyofib is the ratio of subsarcolemmal to total myofibrillar glycogen volume. The weighted mean for each variable was calculated using the assumed distribution of the three fibre types values, and used as a representation of the whole muscle value (see methods section). 


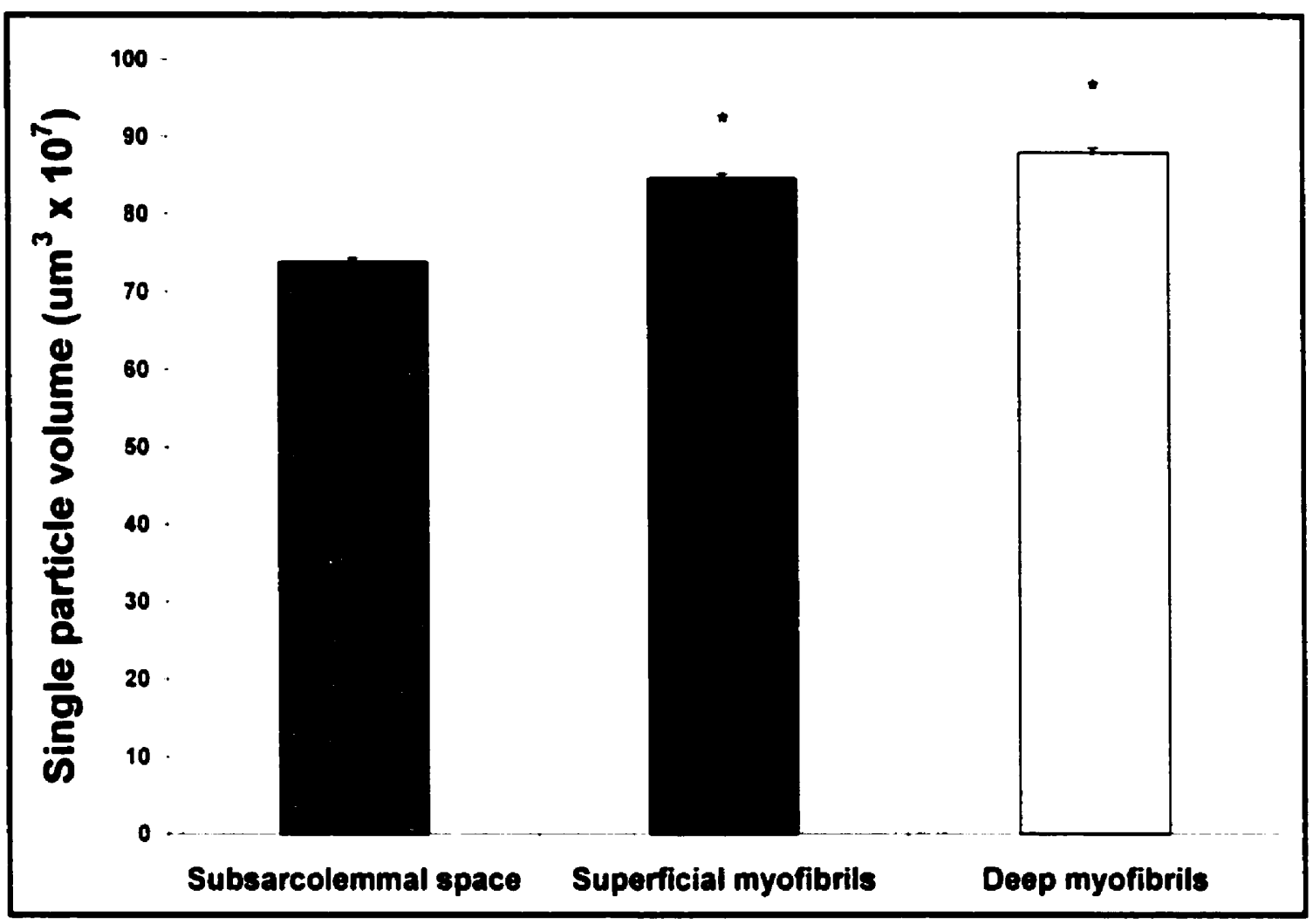

Figure 23. Differences in single particle volume between subcellular locations. Means \pm S.E.M. single particle volume in the three different subcellular locations, and across fibre types. A main effect of subcellular location was observed $(p=0.0001)$. The symbol " $"$ indicates significant difference when compared to the subsarcolemmal location $(p<0.05)$. 


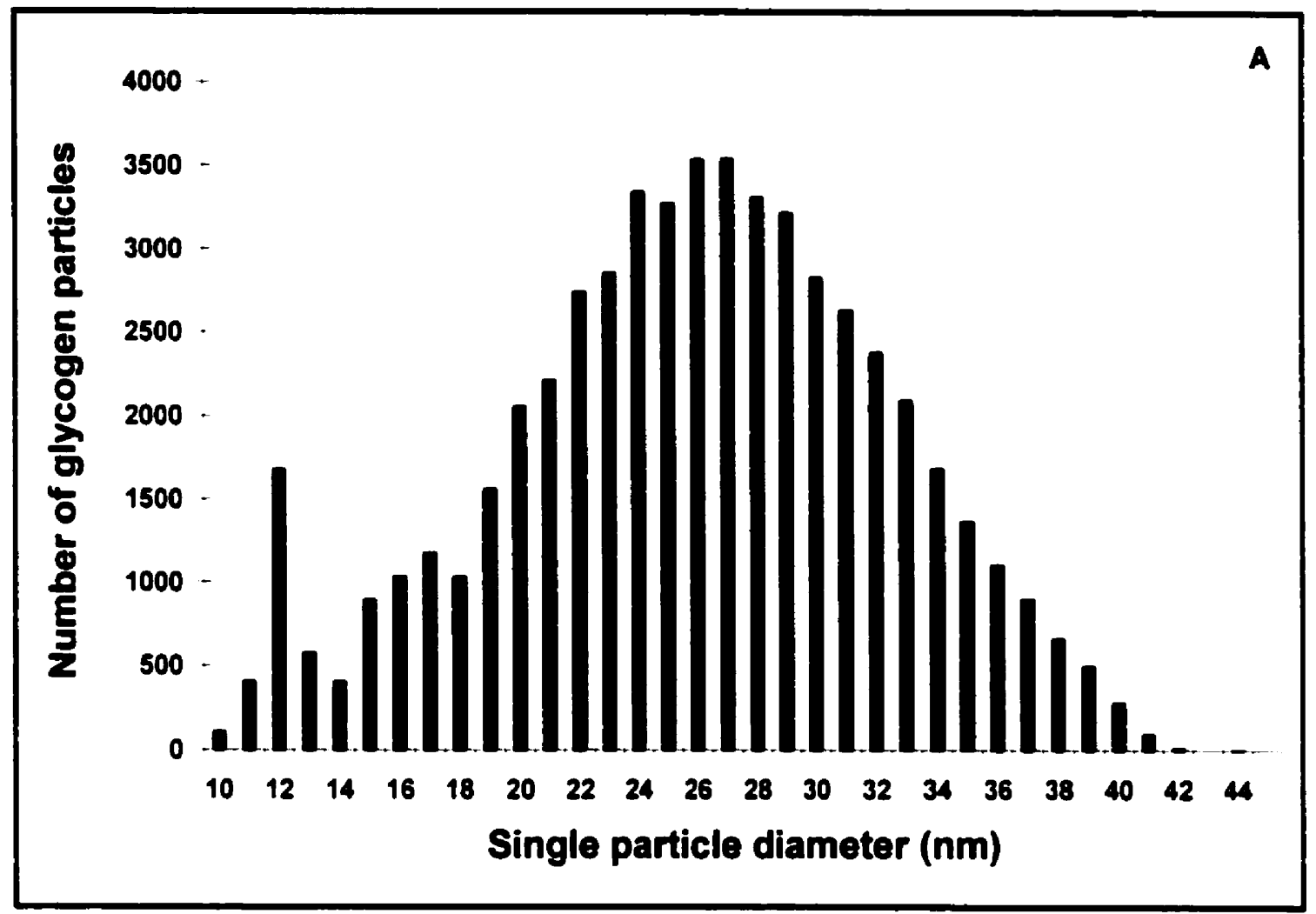

Figure 24 (A). Frequency distribution for the single particle diameters. Single glycogen particle diameter $(\mathrm{nm})$ frequency distribution for 11 subjects. This figure pools the glycogen particies of all images of the 5-6 fibres for each of the 11 subjects, and across fibre types. 


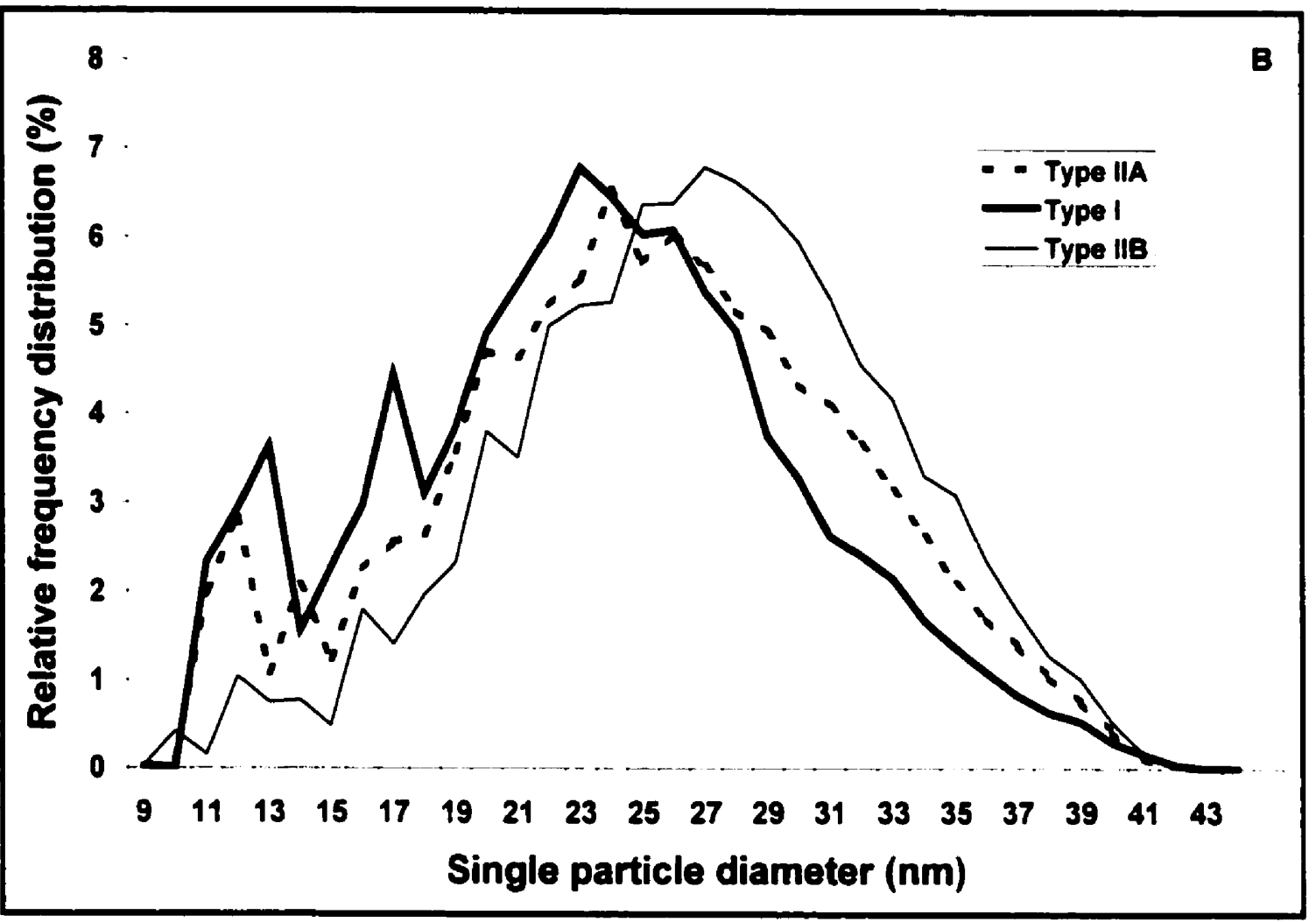

Figure $24(\mathrm{~B})$. Single glycogen particle diameter $(\mathrm{nm})$ relative frequency distribution (\%), for each of the three fibre types. The relative frequency distribution expresses the frequency as a percentage of the absolute frequency. A main effect of fibre type was observed $(P=0.04)$.

The average glycogen volume and number of particles for each fibre type are presented in Table 4. There were no differences among fibre types; however, a main effect of subcellular location was present for both glycogen volume $(p=0.0001)$ and number of glycogen particles $(p=0.0001)$. Figure 25 illustrates the glycogen volume (weighted for estimated percent fibre type complement of the muscle as described in Chapter IV - Detailed Methods) in each of the subcellular locations. While the granule size was smaller in the subsarcolemmal space (Figure 23), the glycogen volume ( $\mu \mathrm{m}^{3}$ of 
glycogen per $\mu \mathrm{m}^{3}$ of muscle tissue), was 59 and $74 \%$ greater in the subsarcolemmal space $(0.037 \pm 0.001)$ than in the deep $(0.023 \pm 0.0002)$ and superficial $(0.021 \pm 0.001)$ myofibrils, respectively (both $p=0.001$ ). While glycogen volume (weighted for estimated fibre type complement) was $10 \%$ greater in the deep compared to the superficial myofibrils, consistent with the single particle volume results (Figure 23), these differences did not reach statistical significance $(p=0.32)$. The number of glycogen particles, as expressed in number per $\mu \mathrm{m}^{3}$ of muscle tissue, followed the same pattern as the glycogen volume and was significantly greater in the subsarcolemmal space $(5152 \pm 332)$ than in the deep $(2741 \pm 146)$ and superficial $(2770 \pm 190)$ myofibrils (both $p=0.0001)$.

133 


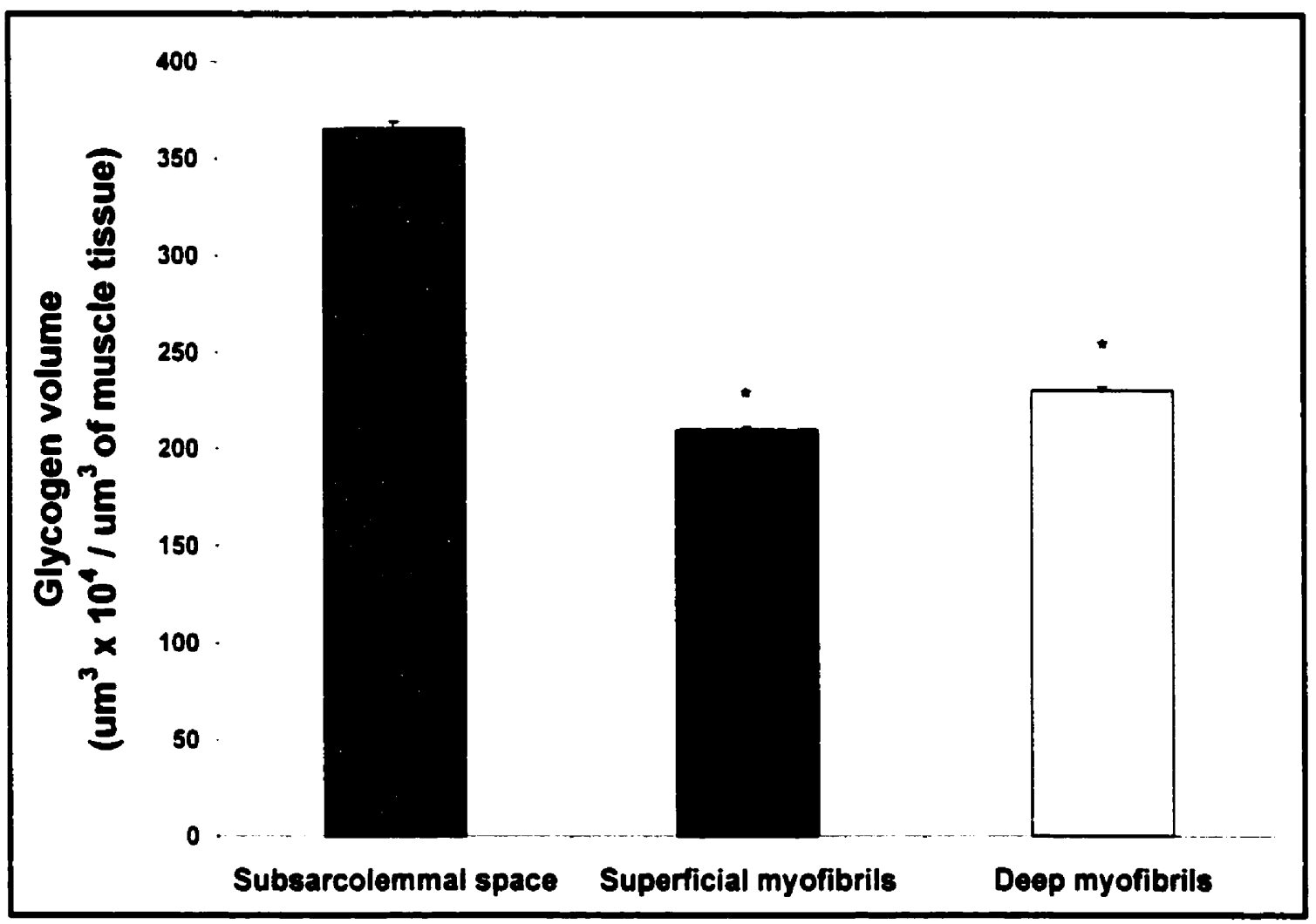

Figure 25. Differences in glycogen volume between subcellular locations. Means \pm S.E.M. glycogen volume in the three different subcellular locations. The glycogen volume is expressed in $\mu \mathrm{m}^{3} \times 10^{4} / \mu$ $\mathrm{m}^{3}$ of muscle tissue. A main effect of subcellular location was observed $(p=0.0001)$. "** indicates a significant difference compared to the subsarcolemmal location $(p<0.05)$.

As expected, the inter-myofibrillar region contained significantly more glycogen than the intra-myofibrillar region. The former represented 86 to $90 \%$ of the myofibrillar glycogen (Table 4). This was observed for both the glycogen volume $(p=0.0001)$ and the number of glycogen particles $(p=0.0001)$, with the individual particle diameter not being different between regions (see above). 
Table 4 summarises the relative proportion of the myofibrillar (myofib) glycogen volume present in the intra-myofibrillar (intra / myofib) region. The type I fibres had relatively more intra-myofibrillar glycogen than the type IIA $(p<0.006)$ and IIB ( $<<$ $0.009)$ fibres.

\section{Discussion}

The main goals of the present study were: a) to quantify the distribution of human skeletal muscle glycogen in different subcellular locations and b) to examine these measures in type I and II fibres. The major findings were: 1) we confirmed the compartmentalised and heterogeneous distribution of human skeletal muscle glycogen, with the glycogen particles being more concentrated in the subsarcolemmal space than in the myofibrillar space, 2) single particle diameters follow a normal distribution pattern 3) single particle volume is greater in the myofibrillar space compared to that in the subsarcolemmal space, and 4) there are significant differences in the subcellular distribution of glycogen between fibre types, particles being larger in type $\|$ than I fibres, and the proportion of intra-myofibrillar to inter-myofibrillar glycogen being greater in type I than II fibres.

\section{Subcellular locations.}

We found the muscle glycogen to be heterogeneously distributed within the cell, in a manner similar to what has been described previously (Friden et al. 1985 and 1989; Schmalbruch and Kamieniecka 1974; Sjostrom et al. 1982). Schmalbruch and Kamieniecka (1974) reported the glycogen granules to be either closely packed in strands marking the boundaries of the myofibrils in the I-band region, or to be located in rows or as single granules within the myofibrils (see Figure 9). Sjostrom and collaborators (1982) extended these observations by reporting a third subcellular site of 
glycogen storage, the subsarcolemmal space. They characterised the intra-muscular distribution of glycogen of their control subjects (i.e. resting) as: 1) large subsarcolemmal accumulations, 2) large accumulations between the myofibrils (especially at I-band in close proximity to the mitochondria and SR), and 3) relatively smaller intra-myofibrillar accumulations. They also reported that the intra-myofibrillar glycogen particles were oriented as though they occupied a space previously filled with a thin or thick myofilament. In addition, Friden and collaborators (1989) further emphasised an I-band based location of glycogen, both for the intra- and intermyofibrillar glycogen, and noted that the intra-myofibrillar glycogen granules were found both in horizontal rows beginning at the lateral border of the I band and extending towards the $M$ line as well as longitudinal rows of single particles bordering the $Z$ line. These observations were apparent in the present study (see figure 9 in Chapter IV Detailed Methods).

These earlier experiments were insightful, but the methodological approach used in estimating muscle glycogen concentration was strictly qualitative. Owing to the labour-intensive nature of TEM methods and the lack of advanced computerised processes to assist in data collection, their sample sizes were small, and sampling was not performed systematically. For example, in the investigations of human skeletal muscle (Friden et al. 1985 and 1989; Sjostrom et al. 1982) the resting controls were 4 or 5 subjects and in one case (Friden et al. 1989) there were only 3 subjects who exercised. Furthermore, the number of fibres sampled was rarely stated nor was there a detailed description of how subcellular sampling sites were determined.

In the present study, we provide the first quantitative analysis of subcellular glycogen location. We confirmed the presence of and quantified the characteristics of three, morphologically distinct compartments in human skeletal muscle: subsarcolemmal, intra-myofibrillar, and inter-myofibrillar spaces. We found that the 
inter-myofibrillar space is the largest glycogen compartment, although the subsarcolemmal space is more densely packed with glycogen granules, having almost twice the granule number per unit area than the myofibrillar space (Figure 25). Our results also confirm that the particles located between the myofilaments (intramyofibrillar region) were either isolated from each other or aligned in strands, but never in clusters. These observations are similar to what has been described previously (Schmalbruch \& Kamieniecka, 1974).

These anatomical compartments probably have metabolic significance. Friden and colleagues (1985) compared the pattern of glycogen depletion during a marathon to that during an intense, anaerobic, sprint exercise. They found that the subsarcolemmal glycogen fraction was not depleted at the end of a marathon while the other locations were "empty". In contrast, in fibres of the three (Friden et el. 1989) and six (Friden et al. 1985) subjects who performed sprints, there was a marked depletion of subsarcolemmal pools, while the "peri-mitochondrial" locations remained intact at fatigue. They concluded that, depending on the type of exercise, differential sequential glycogen utilisation patterns can be observed, and suggested a compartmentalised metabolism of glycogen. The present quantitative technique will facilitate evaluation of their hypothesis that the three subcellular glycogen storage sites represent distinct metabolic compartments.

Our findings represent the first evidence of a graded increase in single glycogen particle size from the sarcolemma to the centre of the muscle fibre (Figure 23). The metabolic implication for this finding is presently unknown. However, a gradient of intermyofibrillar mitochondrial content, from high to low, has previously been reported from the sarcolemma to the centre of the muscle fibre (Kayar et al. 1986). In addition, intermyofibrillar and subsarcolemmal mitochondrial fractions have previously been isolated 
from skeletal muscle (Cogswell et al. 1993). They were shown to have distinct biochemical properties, and to have differential adaptations to increasing or decreasing muscle activity. A quantitative analysis of the physiological responses of the different intra-muscular compartments, with regard to glycogen metabolism, remains to be performed.

Single particle distribution.

The present study demonstrates for the first time that, in resting, human skeletal muscle, the glycogen particles present as a continuum of sizes ranging from 10 to 44 $\mathrm{nm}$ in diameter (Figure $24(\mathrm{~A})$ ). This is in contrast to the description of Friden et al (1989), who reported two distinct populations of glycogen particles, with average diameters of 22 and $56 \mathrm{~nm}$. However, their results are based on the analysis of 144 glycogen particles while we measured more than 55000 granules. The mean single glycogen particle diameter was found to be $25 \mathrm{~nm}$, in agreement with values previously reported (Friden et al. 1985 and 1989; Rybicka 1981a and 1996; Schmalbruch and Kamieniecka 1974; Wanson and Drochmans 1968). The frequency distribution of the single particle diameters from eleven subjects suggests that the distribution is normal, although slightly skewed on the left. The latter finding is to be expected considering the error introduced by the $70 \mathrm{~nm}$ sections relative to the $25 \mathrm{~nm}$ average diameter of the glycogen particles. A proportion of particles will ultimately be cut in such a way that will lead to an underestimation of their actual diameter. That is, if more than $50 \%$ of a spherical granule is not included in the section, then the true diameter will be underestimated. Conversely, if more than $50 \%$ of such a particle is included, the correct diameter will be observed. This bias can only lead to an underestimation of a particle diameter, hence the distribution being skewed on the left. As reported by Williams 
(1977), this only becomes a significant problem when the object being measured has a greater diameter than the thickness of the thin section.

We found that the maximal granule diameter was $44 \mathrm{~nm}$. The extent to which this finding agrees with the values predicted from mathematical modelling is striking. Considerable evidence converges to propose that an optimally efficient glycogen $\beta$ particle would have a maximal diameter of $42 \mathrm{~nm}$ (Goldsmith et al. 1982; MelendezHevia et al. 1993; Melendez et al. 1997). According to the models, the "mature" glycogen $\beta$-particle (macroglycogen) would be a spherical particle arranged in 12 concentric layers (tiers) of carbohydrate, with a molecular mass of $10^{7} \mathrm{Da}$. Each tier is estimated to add $3.8 \mathrm{~nm}$ to the diameter (Goldsmith et al. 1982), and the amount of glucose available in any outer tier is always $34.6 \%$ of the total amount of glucose in the particle (Melendez-Hevia et al. 1993). This means that the differences in granule size between subsarcolemmal and myofibrillar regions and between type I and type II fibres are very important for glycogen storage volume. The dimensions obtained from those models, however, do not include the weight and size of the different enzymes that are known to be associated with the glycogen particle. This could explain the fact that our maximal diameter is slightly larger than the predicted maximal diameter.

There is controversy in the literature as to whether proglycogen and macroglycogen only exist as discrete entities (Alonso et al. 1995) or as a wide range of molecular weights (Skurat et al. 1997). Our data firmly support the theory that there is a continuous spectrum of glycogen particles sizes, ranging from sizes considerably smaller than the upper limit of proglycogen $(\sim 30 \mathrm{~nm})$, to sizes corresponding to the maximal macroglycogen diameter $(-42 \mathrm{~nm})$. 
Fibre types.

The results of the present study suggest that there is no difference in resting muscle glycogen concentration between fibre types (Table 4). Although an earlier study using histochemical methods also reported no difference in resting glycogen levels between fibre types in human (Essen and Henriksson 1974), the majority of studies of human muscle find a 16 to $40 \%$ greater glycogen content in type II compared to type I fibres (Vollestad et al. 1984; Casey et al. 1995; Tsintzas et al. 1996; EsbjornssonLiljedahl et al. 1999). The limitation with regards to our inability to establish a total fibre glycogen concentration may account for the lack of significant differences. Indeed, our comparison of glycogen concentration among fibre types was obtained exclusively from the myofibrillar glycogen and hence, does not include data from the subsarcolemmal space, which has dense glycogen stores. It was not possible to measure the individual muscle fibre circumference and volume, which is essential in caiculating the relative contribution of the subsarcolemmal space to the total fibre volume. Since the diameter of individual muscle fibres is known to range from 10 to $100 \mu \mathrm{m}$ (Landon 1992), the error introduced by an average value would be tremendous. Furthermore, the subsarcolemmal to total myofibrillar ratio, although not significantly different between fibre types, was $21 \%$ greater in type II compared to type I fibres. This suggests that there may be relatively less subsarcolemmal glycogen in type I than type IIA fibres, which would contribute to a difference in total muscle glycogen concentration between fibre types. Our data for fibre types is based on a limited number of cells, due to the very tedious nature of the analysis. It is encouraging that despite these relatively small sample sizes, we were able to detect some differences between type I and type IIA fibres (i.e. single particle volume and intra / myofib). On the other hand, no significant differences were found between type I and type IIB fibres for any of the dependent variables. While this could be real, the low natural frequency of occurrence of type IIB 
fibres in skeletal muscles restricted the statistical power of the tests involving these fibres. It will require further studies to explore this in detail.

We found an effect of fibre type on individual glycogen particle size (Figure 24 (B) and Table 4) such that I < $\| A$ < IIB. This order also represents the natural order of fibre type recruitment during voluntary muscle contraction (Henneman 1957). One could speculate that since type I fibres are being constantly recruited during activities of daily living, their glycogen particles are in a more dynamic state of turnover.

While the type I fibres were found to have smaller glycogen particles, they also tended to have more particles than type IIA and IIB fibres (Table 4). That is, compared to type I fibres, the mean granule numbers were 18 and $25 \%$ lesser in type IIA and IIB, respectively. Hansen et al. (2000) recently reported that rodent muscle type I fibres had more glycogenin than did type II fibres. Glycogenin is the self-glucosylating protein backbone that serves as a primer for glycogen synthesis. Over-expression of glycogenin has been shown to cause a production of more but smaller, glycogen particles (Skurat et al. 1997). While it is speculative, type I fibres in humans may also express more glycogenin and this could explain the greater number of smaller glycogen particles.

Friden and co-workers (1989) suggested that the only consistent difference between type I and II fibres was that more glycogen particles were located at the H-zone in the type II fibres. We did not differentiate between the different intra-myofibrillar locations. However, we found that type I fibres had a significantly greater proportion of myofibrillar glycogen located in the intra-myofibrillar space (Table 4). This finding is in agreement with those of Schmalbruch and Kamieniecka (1974), who observed that a larger proportion of the glycogen in type I fibres to be located between the actin and myosin filaments, and the glycogen in type IIA and IIB fibres to be preferentially 
distributed between myofibrils. Thus we are able to substantiate these earlier descriptions with quantified evidence.

\section{Conclusion}

This study presents the use of a novel method to quantify the subcellular distribution of skeletal muscle glycogen. We confirm that in resting human skeletal muscles the glycogen distribution is compartmentalised and heterogeneous, with the glycogen being more concentrated in the subsarcolemmal space than in the myofibrillar space, and the single particle volume being greater in the latter. We provide direct evidence that the single particle diameters range from 10 to $44 \mathrm{~nm}(25.2 \pm 2.8 \mathrm{~nm})$ and follow a normal distribution. Finally, we found significant differences in the subcellular distribution of glycogen among fibre types, the single particles being larger in type II fibres, and the proportion of intra-myofibrillar glycogen being greater in type I. The results of the present study represent the first quantitative demonstration of a compartmentalised pattern of subcellular glycogen deposition in human skeletal muscles. 


\section{Chapter VI}

Experiment 2: Quantitative analysis of the intracellular distribution of glycogen in human skeletal muscle during recovery from prolonged exercise. 


\section{Introduction}

Numerous, extensive biochemical experiments have provided the basis of our understanding of glycogen metabolism over the past 150 years. Since the classic study by Hultman and Bergstrom (1967), scientists have frequently examined the resynthesis of skeletal muscle glycogen by determining the net changes in total tissue concentrations. The pattern of muscle glycogen synthesis following its depletion by exercise has been shown to be biphasic, with an initial rapid insulin-independent period of synthesis, followed by a slower insulin dependent rate of synthesis (Price et al. 1994). While it is known that glycogen exists as individual particles located in distinct subcellular locations / compartments (Friden et al. 1985 and 1989; Marchand et al. 2001), there has been no study to characterise the pattern by which glycogen is resynthesised in terms of single particle sizes, numbers, and subcellular locations.

We recently developed a method to quantify the subcellular distribution of skeletal muscle glycogen (Marchand et al. 2001), and confirmed that resting human skeletal muscle glycogen distribution is compartmentalised and heterogeneous with respect of its pattern of distribution (Friden et al. 1985 and 1989; Sjostrom et al. 1982). Furthermore, in agreement with the available literature (Biava 1963; Revel 1964; Rybicka 1996), the study showed that the single particle ( $\beta$ particle) sizes range from 10 to $44 \mathrm{~nm}(25.2 \pm 2.8 \mathrm{~nm})$, that they follow a normal distribution. In addition, we found the glycogen particles to be significantly smaller and more numerous in the subsarcolemmal compared to the myofibrillar subcellular compartments. Finally, we observed significant differences in the subcellular distribution of glycogen between fibre types (Marchand et al. 2001).

Ekblom and colleagues are the only group to have examined the impact of different types of exercise on the subcellular location of human skeletal muscle glycogen 
(Friden et al. 1985 and 1989; Sjostrom et al. 1982). Despite the qualitative nature of their analyses, their results strongly suggest that the metabolism of glycogen varies dramatically in different subcellular locations. Prolonged ( 30 and $42 \mathrm{~km}$ ) moderateintensity running was demonstrated to lead to the extensive depletion of the glycogen in the core of the depleted fibres, and the particles remaining were found to be of smaller sizes. Interestingly, the subsarcolemmal glycogen was found to be extensively depleted in one study (Sjostrom et al. 1982), and relatively less utilised in the other study, where the particles in that location were reported to retain their size and shape (Friden et al. 1985). On the other hand, they showed short-term intense anaerobic exercise to lead to the extensive depletion of subsarcolemmal glycogen (Friden et al. 1985 and 1989).

Based on these results, Friden and colleagues (1985 and 1989) proposed that the subsarcolemmal glycogen particles may be stored there to secure energy for the maintenance of the plasma membrane potential and / or to act as an intra-cellular reserve energy supply. Accordingly, they proposed that during high-intensity exercise, the circulating energy pools are not sufficient for both the plasma membrane and contraction functions. Therefore, the subsarcolemmal glycogen stores would be utilised to yield ATP for the $\mathrm{Na}^{+} / \mathrm{K}^{+}$pump (Friden et al. 1985 and 1989). A quantitative approach is required to verify these qualitative findings, and to examine the postexercise pattern of glycogen resynthesis.

The purpose of the present study was therefore to quantify the intra-cellular glycogen distribution in human skeletal muscles during the 48-hour recovery period from a moderate intensity, glycogen-depleting exercise. The pattern of post-exercise glycogen metabolism was examined with respect to particle sizes, number and volume, and compared between subcellular locations. Based on the available literature, we hypothesised that exercise will lead to the preferential catabolism of myofibrillar over the 
subsarcolemmal glycogen. The paucity of previous investigations limited our ability to put forward a detailed hypothesis regarding the pattern of glycogen resynthesis following its depletion by exercise. Therefore, we proposed to determine the post-exercise pattern of glycogen resynthesis with respect to glycogen particle size, number, and subcellular compartments.

\section{Methods}

Subjects. The study was approved by the University of Guelph's Human Ethics Committee and conformed to the standards set by the Declaration of Helsinki. Five male subjects were informed in writing about the nature of the experiment, volunteered to participate, and signed a consent form. The mean ( \pm S.E.M.) age, height, weight, and $\mathrm{VO}_{2} \mathrm{max}$ of the subjects were $25 \pm 1$ years old, $182 \pm 3 \mathrm{~cm}, 79 \pm 2 \mathrm{~kg}$, and $58 \pm 2 \mathrm{~mL}$. $\mathrm{kg}^{-1} \cdot \min ^{-1}$, respectively.

Pre-trial procedures. The subjects initially underwent an incremental $\mathrm{VO}_{2}$ max test on an electronically braked cycle ergometer (Quinton Excaliber). A week before the trial, a practice ride at $\sim 70 \% \mathrm{VO}_{2} \max$ was completed. This ride served two purposes: (1) confirmation of the exercise workload intensity and (2) familiarisation of the subjects to the workload and experimental protocol.

Experimental protocol. Subjects arrived in the morning at the testing centre and completed an exhaustive glycogen depletion ride at $70 \% \mathrm{VO}_{2} \max$. Exhaustion was determined as the point at which subjects could no longer maintain the pedalling frequency at a constant resistance. At exhaustion, a muscle biopsy $(0 \mathrm{~h})$ was taken from the vastus lateralis muscle by use of the percutaneous needle biopsy technique 
(Hultman 1967). Muscle biopsies were subsequently obtained at 4, 24, and $48 \mathrm{~h}$ following exhaustion.

Analyses, calculations and statistics.

Please refer to Chapter IV - Detailed Methods.

\section{Results}

Biochemical data.

The subjects had an average ( \pm S.E.M.) total muscle Gt of $36.3 \pm 8.3,94.5 \pm$ $12.0,278.5 \pm 55.7$, and $374.3 \pm 60.7$ glucosyl units $\bullet \mathrm{kg}^{-1} \mathrm{dw}$, for times $0,4,24$, and 48 $h$, respectively. In addition, they had an average of $85 \pm 7,89 \pm 2,80 \pm 5$, and $64 \pm 4 \%$ of their total glycogen in the form of PG, for times $0,4,24$, and $48 \mathrm{~h}$, respectively.

TEM data.

Time effect.

Table 5 summarises the time, fibre type, and time $x$ fibre type effects on all dependent variables. For each time, the weighted means were obtained from all three fibre types (Chapter IV - Detailed Methods) and used as a total muscle value. As expected, all variables measured (except for subsarcolemmal number of glycogen particles; $p=0.07)$ were significantly affected by time $(p<0.05)$.

The effect of time on myofibrillar glycogen volume, myofibrillar number of glycogen particles, and single glycogen particle volume is illustrated in Figure 26. All three variables increased with time $(p<0.0001)$. Early in the recovery $(0-4 h)$, the increase in glycogen volume was produced by a massive increase in number of glycogen particles ( $186 \%$ ) combined with a non-significant increase in single particle 
volume ( $80 \%$ ). From 4 to $24 \mathrm{~h}$ of recovery, the increase in glycogen volume was entirely caused by an increase in the size of the individual glycogen particles (160\%), since the number of glycogen particles reached a plateau at $4 \mathrm{~h}$ and remained significantly unchanged until $48 \mathrm{~h}$ post-exercise. Furthermore, from 24 to $48 \mathrm{~h}$, the increase in glycogen was due to the combination of non-significant increases in both number of glycogen particles (24\%) and single particle size (16\%).

From 0 to $48 \mathrm{~h}$ post-exercise, the myofibrillar and subsarcolemmal glycogen volume increased by $1256 \%$ and $872 \%$, respectively (Table 5). This greater relative increase in myofibrillar compared to subsarcolemmal glycogen volume fraction is reflected by a decrease in the Subsarc / Tmyofib ratio from 3.3 at $0 \mathrm{~h}$, to 1.6 at $48 \mathrm{~h}$ post-exercise. In absolute terms however, the myofibrillar and the subsarcolemmal glycogen increased by 201 and $314 \mu \mathrm{m}^{3} \times 10^{4} / \mathrm{um}^{3}$ of muscle, respectively, over the entire recovery period. This suggests a greater absolute ability for glycogen to be synthesised in the subsarcolemmal compared to the myofibrillar space. Figure 27 illustrates the net rate of synthesis in both glycogen locations. While the net rates of glycogen synthesis decreased with time in both fractions $(p=0.009)$, the rate was significantly greater in the subsarcolemmal compared to the myofibrillar space $(p=$ 0.01). No difference was found between the net glycogen synthesis rates between the deep and superficial myofibrils, so the data were pooled as "total myofibrillar" in Figure 27. Post hoc analysis revealed the net subsarcolemmal glycogen synthesis rate to be greater than the net total myofibrillar rate during initial recovery $(0-4 \mathrm{~h})$, but not different from 4 to $48 \mathrm{~h}$. Similarly, the biochemically determined net rates of synthesis decreased with time for both Gt $(p=0.003)$ and PG $(p<0.001)$. The net rate of MG synthesis did not change over time.

Table 5 summarises the relative proportion of myofibrillar glycogen located in the intramyofibrillar region (Intra / myofib). Early in the exercise recovery period, less than $4 \%$ 
of the total myofibrillar glycogen was located within the myofibrils while after $48 \mathrm{~h}$ of recovery, more than $14 \%$ was located in the intra-myofibrillar region. Taken together, these results suggest that not only is the intra-myofibrillar glycogen preferentially used during exercise, but that it regenerates faster than the inter-myofibrillar glycogen as its proportion increased to $12 \%$ of myofibrillar glycogen at 4 hours. Furthermore, as more glycogen accumulates in the muscle, a greater proportion of it is deposited in between the actin and myosin myofilaments (intra-myofibrillar). 
Table 5. Summary of the dependent variables.

\begin{tabular}{|c|c|c|c|c|c|c|c|c|c|}
\hline Time & $\begin{array}{l}\text { Flbar } \\
\text { type }\end{array}$ & & $\begin{array}{l}\text { Myofibrillar } \\
\text { glycogen volume } \\
\text { (um } \times 10^{3} / m^{3} \text { of musela) }\end{array}$ & $\begin{array}{l}\text { Myoribrillar } \\
\text { " particles } \\
\text { (e/um' of muselo) }\end{array}$ & $\begin{array}{l}\text { Subsarcolemmal } \\
\text { glycogen volume } \\
\text { (um } \mathrm{sm}^{2} \times 10^{2} \mathrm{~mm}^{2} \text { of muacle) }\end{array}$ & $\begin{array}{c}\text { Subsarcolemmal } \\
\text { particles } \\
\text { (0 tum of muscis) }\end{array}$ & $\begin{array}{l}\text { Particle } \\
\text { volume } \\
\left(4 \mathrm{~m}^{3} \times 10^{\prime}\right)\end{array}$ & $\begin{array}{l}\text { Subsarl } \\
\text { Tmyofib } \\
\text { (Units) }\end{array}$ & $\begin{array}{l}\text { Intor I } \\
\text { myoft } \\
\text { (x) }\end{array}$ \\
\hline \multirow[t]{7}{*}{ on } & I & Moan & 4.0 & 384 & 9.8 & 928 & 12.2 & 3.3 & $t$ \\
\hline & & S.E.M. & $\begin{array}{l}3.2 \\
\text { (d) }\end{array}$ & $\begin{array}{l}322 \\
\text { (d) }\end{array}$ & 7.4 & 676 & 1.9 & 1.8 & 1 \\
\hline & IIA & Maan & 12.8 & 1200 & 47.4 & 4423 & 10.8 & 3.4 & 6 \\
\hline & & S.E.M. & $\begin{array}{l}2.0 \\
\text { (d) }\end{array}$ & $\begin{array}{l}190 \\
\text { (d) }\end{array}$ & 14.4 & 1447 & 0.6 & 0.8 & 2 \\
\hline & 118 & Mean & 98.4 & 3403 & 142.5 & 5406 & 31.7 & 1.5 & 8 \\
\hline & & S.E.M. & 53.3 & 244 & 80.5 & 886 & 19.3 & 0.3 & 5 \\
\hline & w.M. & $\begin{array}{l}\text { Moan } \\
\text { S.E.M. }\end{array}$ & $\begin{array}{c}15.7 \\
5.3 \\
(1 \circ e)\end{array}$ & $\begin{array}{c}939 \\
256 \\
(0 \circ \mathrm{c})\end{array}$ & $\begin{array}{l}35.9 \\
12.8 \\
(b e)\end{array}$ & $\begin{array}{l}2702 \\
1161 \\
(a) \text { c) }\end{array}$ & $\begin{array}{c}13.6 \\
1.4 \\
(b \in)\end{array}$ & $\begin{array}{c}3.3 \\
0.8 \\
(0 b e)\end{array}$ & $\begin{array}{c}3 \\
1 \\
(a b c)\end{array}$ \\
\hline \multirow[t]{8}{*}{$4 h$} & $\mathbf{I}$ & Mean & 56.9 & $207 t$ & 117.7 & 4770 & 27.3 & 2.1 & 12 \\
\hline & & S.E.M. & 8.1 & $\begin{array}{l}170 \\
\text { (d) }\end{array}$ & 4.9 & 238 & 2.5 & 0.2 & 4 \\
\hline & $\| \mathbf{A}$ & Moan & 68.6 & 2943 & 123.7 & 5672 & 24.2 & 1.9 & 10 \\
\hline & & S.E.M. & 6.7 & $\begin{array}{l}222 \\
\text { (d) }\end{array}$ & 13.9 & 514 & 2.4 & 0.2 & 1 \\
\hline & It8 & Mean & 71.3 & 2896 & 100.1 & 4565 & 24.5 & 1.5 & 8 \\
\hline & & S.E.M. & 17.1 & 98 & 19.1 & 772 & 5.6 & 0.3 & 2 \\
\hline & w.M. & Man & 63.0 & 2687 & 109.9 & 5126 & 24.4 & 1.9 & 12 \\
\hline & & S.E.M. & $\begin{array}{c}7.1 \\
(b \quad t)\end{array}$ & 334 & $\begin{array}{l}18.4 \\
(b c)\end{array}$ & 498 & $\begin{array}{c}3.9 \\
\text { (b c) }\end{array}$ & 0.1 & 2 \\
\hline \multirow[t]{8}{*}{$24 h$} & 1 & Mean & 143.8 & 2470 & 215.5 & 4457 & 58.6 & 1.6 & 14 \\
\hline & & S.E.M. & 15.1 & 143 & 12.4 & 644 & 6.2 & 0.3 & 1 \\
\hline & IIA & Mean & 147.6 & 2280 & 210.2 & 3477 & 66.9 & 1.4 & 7 \\
\hline & & S.E.M. & 11.5 & 158 & 22.9 & 329 & 5.1 & 0.1 & 1 \\
\hline & $\mathbf{1 1 B}$ & Moan & 153.1 & 2153 & 210.6 & 3207 & 74.8 & 1.4 & 6 \\
\hline & & S.E.M. & 15.1 & 253 & 30.1 & 690 & 10.5 & 0.2 & 1 \\
\hline & W.M. & Moan & 146.2 & 2364 & 212.6 & 3969 & 63.0 & 1.5 & 10 \\
\hline & & S.E.M. & $\begin{array}{c}19.1 \\
\text { (c) }\end{array}$ & 202 & $\begin{array}{c}29.9 \\
\text { (c) }\end{array}$ & 470 & 7.8 & 1.0 & $\begin{array}{c}1 \\
\text { (c) }\end{array}$ \\
\hline \multirow[t]{7}{*}{$46 h$} & 1 & $\begin{array}{l}\text { Mean } \\
\text { S.E.M. }\end{array}$ & $\begin{array}{c}231.1 \\
50.8\end{array}$ & $\begin{array}{l}3110 \\
415\end{array}$ & $\begin{array}{c}356.5 \\
71.8\end{array}$ & $\begin{array}{c}5520 \\
678\end{array}$ & $\begin{array}{c}70.2 \\
9.2\end{array}$ & $\begin{array}{l}1.6 \\
0.2\end{array}$ & $\begin{array}{c}19 \\
4\end{array}$ \\
\hline & IIA & Man & 193.7 & 2729 & 321.7 & 5066 & 72.5 & 1.7 & $1 t$ \\
\hline & & S.E.M. & 12.8 & 146 & 26.3 & 302 & 4.4 & 0.1 & 1 \\
\hline & 118 & Mean & 237.3 & 2908 & 304.8 & 4215 & 81.6 & 13 & 8 \\
\hline & & S.E.M. & 72.0 & 585 & 98.0 & 1123 & 15.8 & 0.3 & 5 \\
\hline & w.m. & Moen & 217.0 & 2920 & 350.0 & 5342 & 72.8 & 1.6 & 14 \\
\hline & & S.E.M. & 33.7 & 242 & 55.0 & 387 & 9.4 & 0.1 & 3 \\
\hline \multicolumn{3}{|l|}{$\pi m e$} & $p<0.0001$ & $p<0.0000$ & $p<0.0001$ & $p=0.07$ & $p<0.0001$ & $p=0.02$ & $p<0.006$ \\
\hline \multicolumn{3}{|c|}{ Fiber type } & $p<0.05$ & $p=0.03$ & ns & MS & $p=0.02$ & NS & $p \neq 0.10$ \\
\hline \multicolumn{3}{|c|}{ Time x Fiber type } & $p=0.08$ & $p=0.001$ & ns & MS & MS & NS & $p=0.06$ \\
\hline
\end{tabular}

a: different from $4 h(p<0.05)$. b: different from $24 h(p<0.05)$. c: different from $48 h(p<0.05)$. d: different from type IIB fibres $(p<0.05)$. The symbols of significance are located below the appropriate values. The statistical values for main effects and interactions are presented at the bottom of the table. The "particle volumes" represent the total fibre average. The weighted mean represents the weighted average from all fibre types (described in the text), and was used to evaluate the main effect of time. Subsarc / Tmyofib is the ratio of subsarcolemmal to total myofibrillar glycogen volume. Intra / myofib is the relative proportion of myofibrillar glycogen located in the intra-myofibrillar region. 


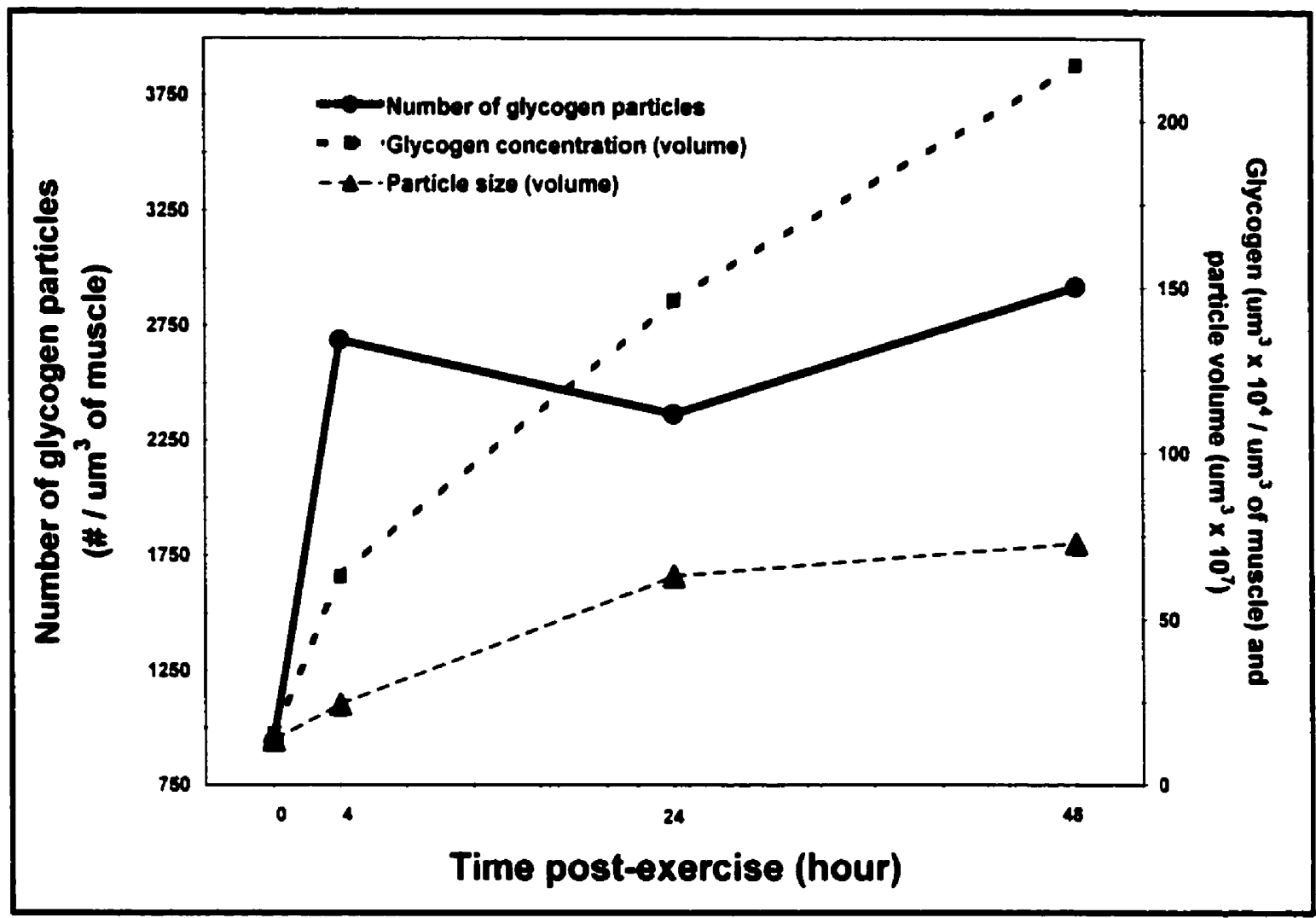

Figure 26. Effect of time post-exercise on total myofibrillar glycogen concentration $\left(\mu \mathrm{m}^{3} \times 10^{4} / \mu \mathrm{m}^{3}\right.$ of muscle tissue), number of glycogen particles ( $\# / \mu \mathrm{m}^{3}$ of muscle tissue), and single glycogen particle size $\left(\mu \mathrm{m}^{3} \times 10^{7}\right)$. The glycogen concentration was multiplied by $10^{4}$ and the single particle volume by $10^{7}$ in order to facilitate the scaling of the graph. Values are weighted means from all three fibre types (see calculation section). The S.E.M. was not presented for sake of clarity. A main effect of time was present for all three variables $(p<0.0001, p<0.0008, p<0.0001$, respectively). The results from the post hoc analysis are reported in Table 5. 


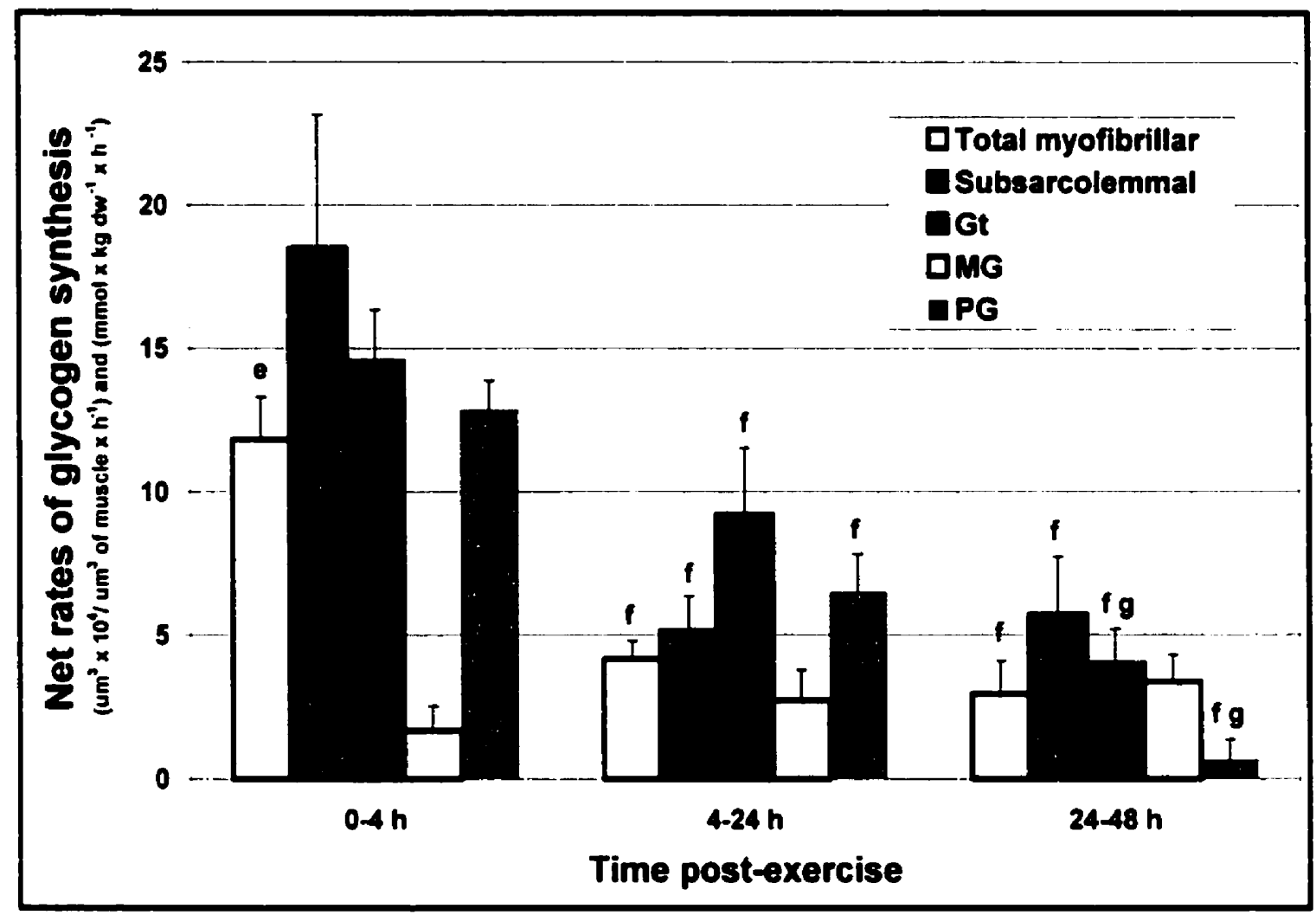

Figure 27. Effect of time post-exercise on the net rates of glycogen synthesis for the myofibrillar space $\left(u m^{3} \times 10^{4} / \mathrm{um}^{3}\right.$ of muscle $\left.\times \mathrm{h}^{-1}\right)$, subsarcolemmal space $\left(\mathrm{um}^{3} \times 10^{4} / \mathrm{um}^{3}\right.$ of muscle $\left.\times \mathrm{h}^{-1}\right)$, total glycogen $(G)$ (mmol $\left.\times \mathrm{kg} \mathrm{dw}^{-1} \times \mathrm{h}^{-1}\right)$, macro-glycogen (MG) (mmol $\times \mathrm{kg} \mathrm{dw}^{-1} \times \mathrm{h}^{-1}$ ) and pro-glycogen $(P G)\left(m m o l \times \mathrm{kg} \mathrm{dw}^{-1} \times \mathrm{h}^{-1}\right)$. The latter three were measured by enzymatic based assays (see methods). Values are means \pm S.E.M. The statistical analyses were carried out separately for the TEM and biochemical data. Main effects of time $(p=0.008)$ and subcellular locations $(p=0.01)$ were present for TEM data. Main effect of time $(p=0.003)$ was present for $G$ and PG. e: significantly different from the subsarcolemmal rate $(p<0.05)$. f. significantly different from rate between $0-4 \mathrm{~h}(p<$ 0.05). g: significantly different from rate between $4-24 h(p<0.05)$. 
Fibre type effect.

Main effects for fibre types were present for particle volume $(p=0.02)$, as well as for myofibrillar glycogen $(p<0.05)$ and number of glycogen particles $(p=0.03)$ (Table

5). As would be expected, the prolonged moderate intensity exercise appeared to result in selective glycogen depletion in type I and IIA fibres, compared to type IIB fibres. The difference between fibre types was only present at time $0 \mathrm{~h}$ for glycogen content, and at time 0 and $4 \mathrm{~h}$ for the number of particles. Hence, for both of these parameters, no effect of fibre type was found after $4 \mathrm{~h}$ of recovery. On the other hand, throughout the entire recovery period (data not shown), the single particle size (volume) was significantly greater in type IIB $\left(53.1 \pm 14.6 \times 10^{7} \mu \mathrm{m}^{3}\right)$ compared to either type IIA (43.6 $\left.\pm 15.4 \times 10^{7} \mu \mathrm{m}^{3}\right)$ or type I $\left(42.1 \pm 13.5 \times 10^{7} \mu \mathrm{m}^{3}\right)$ fibres.

A strong tendency for the Intra / myofib to be dependent on fibre type was also present $(p=0.10)$. The prolonged exercise induced preferential depletion of type I and intra-myofibrillar glycogen resulted in the Intra / myofib to be particularly reduced immediately post-exercise in type I fibres $(1 \pm 1 \%)$. This likely confounded the statistics for fibre type since the Intra / myofib was actually greater in type I than any of the type II fibres from 4 to $48 \mathrm{~h}$ of recovery $(p=0.004)$. Indeed, $48 \mathrm{~h}$ post-exercise, the proportion of myofibrillar glycogen in the intra-myofibrillar region was 1.7 and 2.4 times greater in type I than IIA and IIB (both $p<0.05$ ), respectively.

Single particle distribution.

Figure 28 illustrates the relative frequency distribution for single particle diameter at the four different time points. The relative frequency expresses the frequency as a percentage of the absolute frequency. Throughout the entire recovery, a continuum in particle diameter distribution can be observed. From 0 to $48 \mathrm{~h}$ post-exercise, a gradual 
shift to the right occurs in the distribution frequency curves, with minimal and maximal detectable values of 8 and $43 \mathrm{~nm}$, respectively. The mean particle diameter $(\mathrm{nm})$ for 0 , 4,24 , and $48 \mathrm{~h}$ were $13.3,16.5,22.7$, and 23.8 , respectively $(p<0.0001)$.

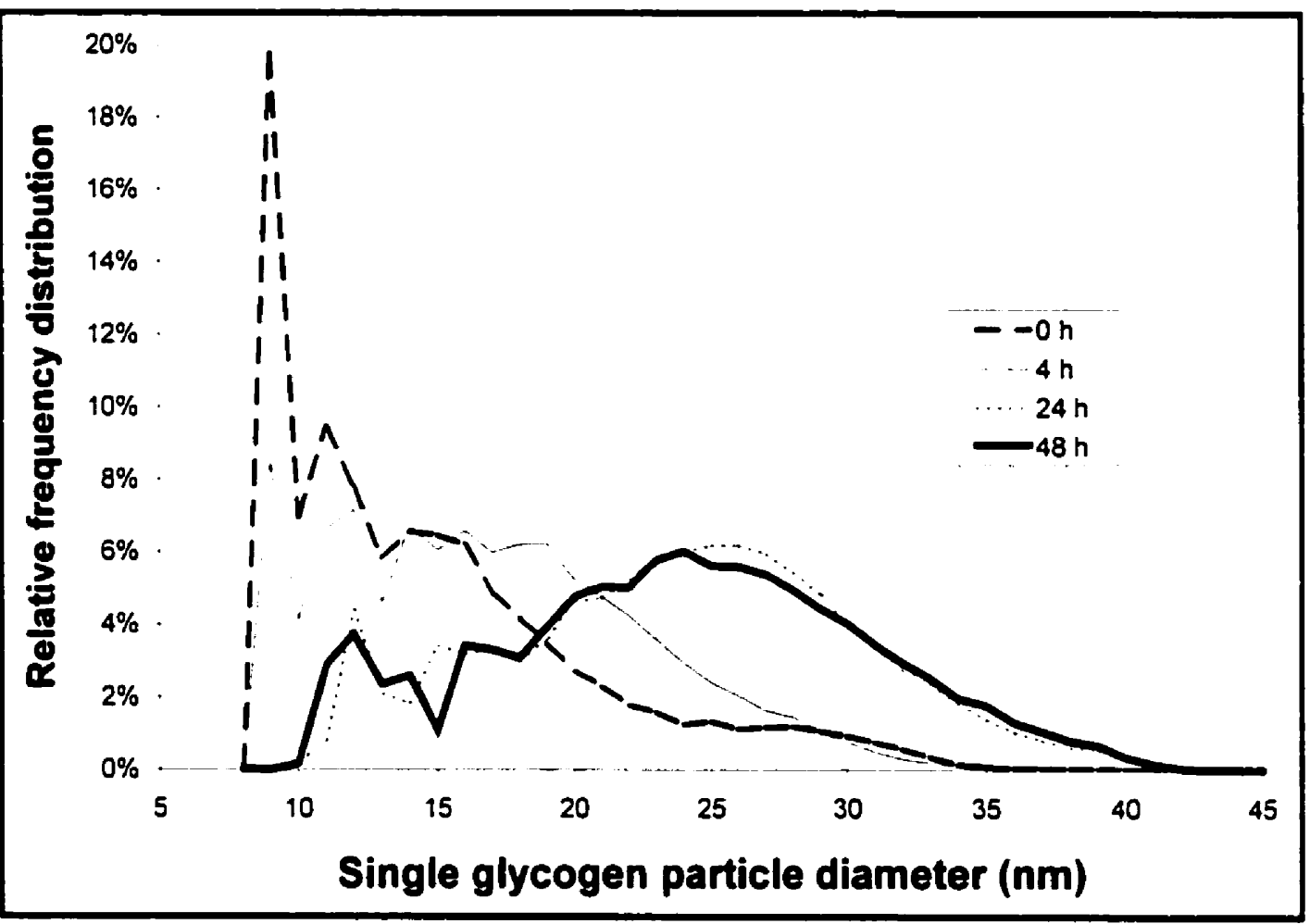

Figure 28. Relative frequency distribution of single particle diameter $(n \mathrm{~nm})$ at the four different times post-exhaustion $(0,4,24$, and $48 \mathrm{~h})$. The relative frequency distribution expresses the frequency as a percentage of the absolute frequency. The data from all five subjects were included ( $n=180000$ glycogen particles). Note the continuous range of particle diameter $(8-43 \mathrm{~nm})$ and the right hand-side shift in distribution with time of recovery. 


\section{Subcellular location effect.}

Figures $29 \mathrm{~A}$ and B illustrate the glycogen concentration (volume) and glycogen particle size (volume) for each of the three subcellular locations (subsarcolemmal, superficial myofibrillar, and deep myofibrillar). Both variables were affected by subcellular location (both $p<0.001$ ). The glycogen concentration was significantly greater in the subsarcolemmal space compared to both the superficial and deep myofibrils, at every time point during recovery (Figure $29(A)$ ). On the other hand, despite this greater subsarcolemmal glycogen volume, the individual glycogen particle volume was similar between subsarcolemmal and myofibrillar locations in the first half of recovery, and was smaller in the subsarcolemmal compared to the myofibrillar compartment at 24 and $48 \mathrm{~h}$ of recovery $(p<0.05)$ (Figure $29(B)$ ). In agreement with these paradoxical findings, the greater glycogen volume in the subsarcolemmal space during the entire recovery was associated with approximately twice the number of glycogen particles (per $\mu \mathrm{m}^{3}$ of muscle) in the subsarcolemmal compared to the total myofibrillar location (deep and superficial myofibrils combined) (Table 5). For both glycogen and single particle volume, post hoc analysis did not detect any significant difference between the two myofibrillar locations throughout the entire recovery. 


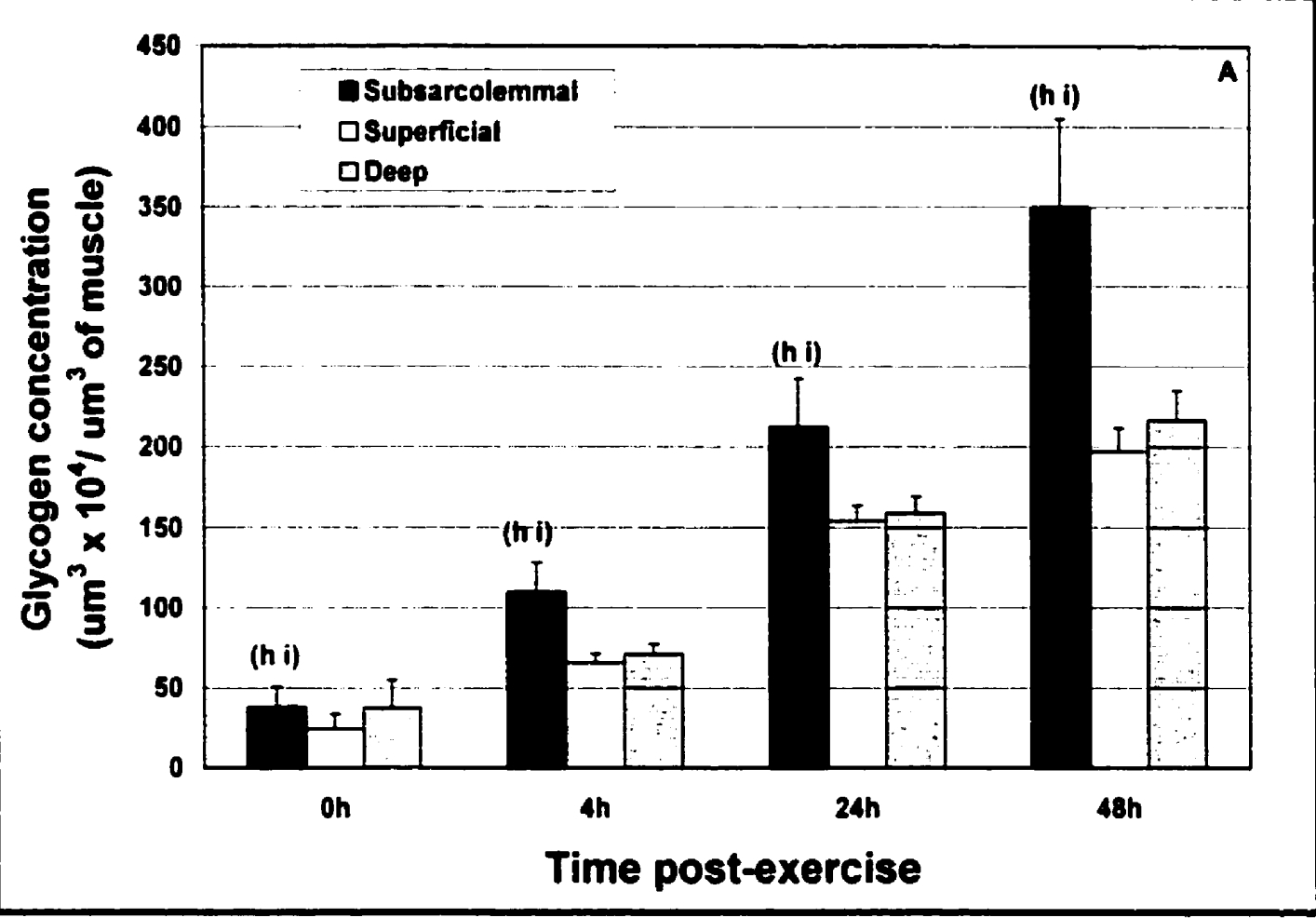

Figure 29 (A). Effect of subcellular locations on glycogen concentration ( $\mu \mathrm{m}^{3} \times 10^{4} / \mu \mathrm{m}^{3}$ of muscle). Values are means \pm S.E.M. A main effect of subcellular location was present for both variables $(p<$ $0.0001)$. h: significantly different from superficial myofibrils $(p<0.05)$. i: significantly different from deep myofibrils $(p<0.05)$. 


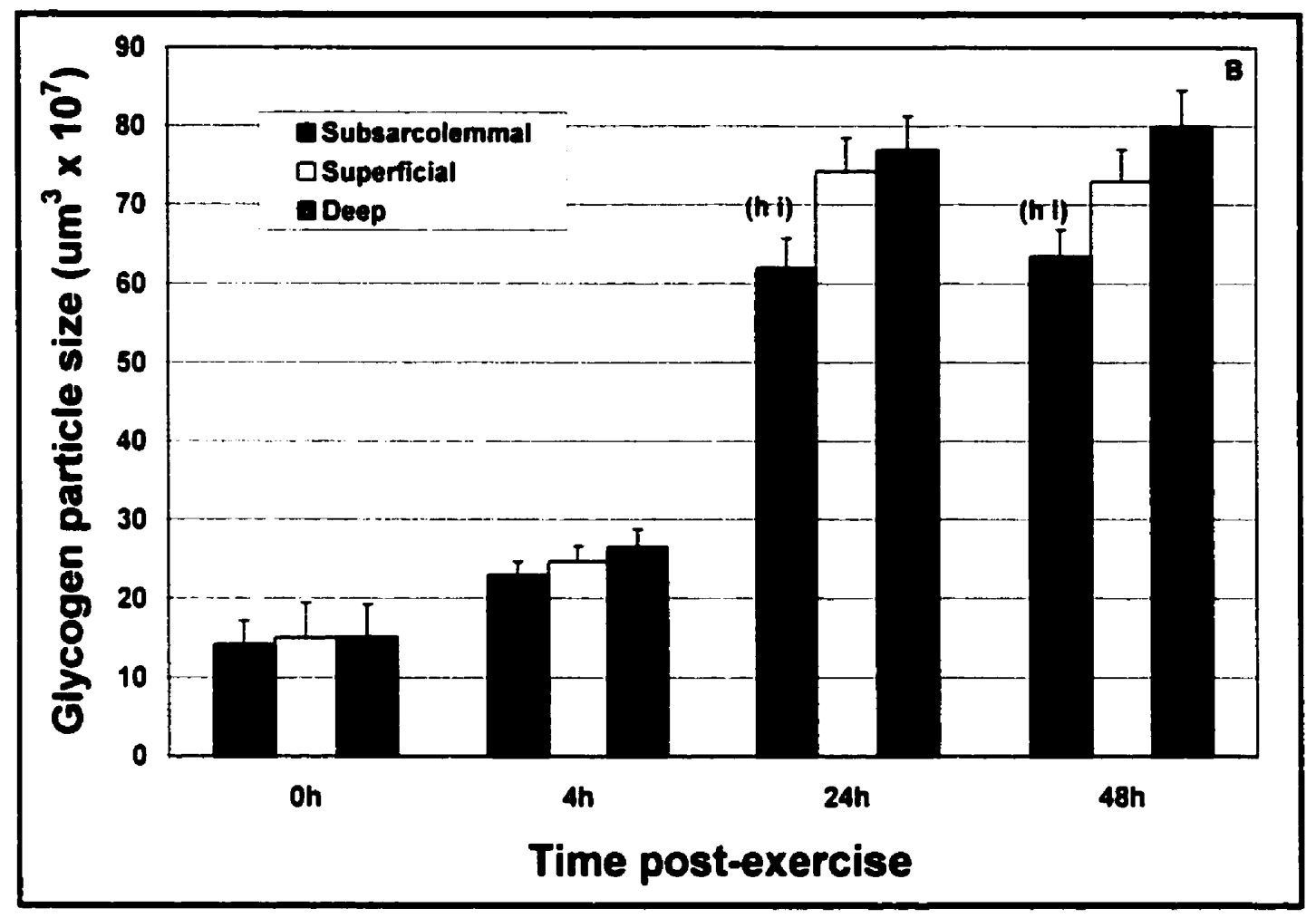

Figure 29 (B). Effect of subcellular locations on single glycogen particle size $\left(\mu \mathrm{m}^{3} \times 10^{7}\right)$ during recovery from a glycogen-depleting exercise. Values are means \pm S.E.M. A main effect of subcellular location was present for both variables $(p<0.0001)$. $h$ : significantly different from superficial myofibrils $(p<0.05)$. i: significantly different from deep myofibrils $(p<0.05)$. 
Figure 30 illustrates the net rate of change of single glycogen particle volume.

The ranked order in net synthesis rate of particle volume was subsarcolemmal < superficial myofibrils $<$ deep myofibrils $(p<0.0001)$. The order was maintained throughout the entire recovery and post hoc analysis revealed the levels of significance across time to be: deep myofibrillar > superficial myofibrillar $(p<0.0007)$; deep myofibrillar > subsarcolemmal $(p<0.0001)$; and superficial myofibrillar > subsarcolemmal $(p<0.0004)$. Interestingly, this order is in contrast to the net rates of synthesis for glycogen concentration (Figure 27), for which the subsarcolemmal location was found to have a greater rate of net glycogen synthesis than the myofibrillar location. Furthermore, the net particle volume synthesis rate was found not to change significantly with time $(p>0.05)$, while net glycogen synthesis rates were found to decrease with time for both subsarcolemmal and myofibrillar fractions. 


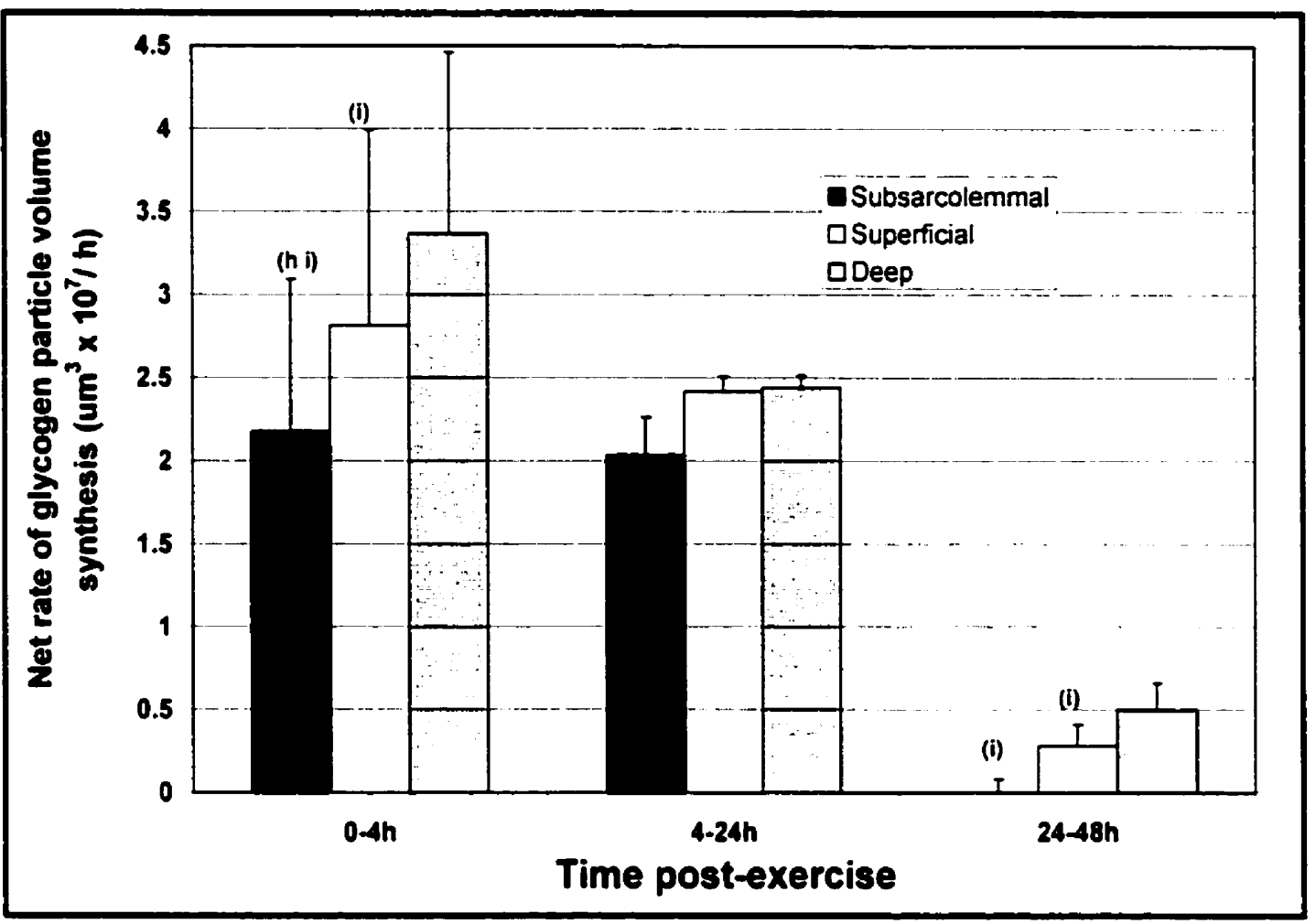

Figure 30. Effect of time and subcellular location on the net rate of single glycogen particle volume synthesis $\left(\mu \mathrm{m}^{3} \times 10^{7} / \mathrm{h}\right)$ following a glycogen-depleting exercise. Values are means \pm S.E.M. A main effects was found for subcellular location $(p=0.0001)$, but not time $(p>0.05)$. $h$ : significantly different from superficial myofibrils $(p<0.05)$. i: significantly different from deep myofibrils $(p<0.05)$.

Illustration of the findings.

Figure 31 illustrates images of myofibrillar muscle tissue obtained from the TEM. Note that the subsarcolemmal space is not included in these images. They represent muscle from the same subject, at each of the different recovery time points. Immediately post-exercise (A), the typical image is less clear, possibly due to the increase in cellular volume secondary to the exercise. The myofibrillar glycogen is sparse, and almost exclusively located in the inter-myofibrillar region, associated with 
the I-band. Four hours later (B), more myofibrillar glycogen can be seen in the form of a greater number of small glycogen particles. The glycogen is distributed in both myofibrillar regions, but a relatively greater proportion of it is located in the intramyofibrillar region compared to time $0 \mathrm{~h}$. In both myofibrillar regions, the glycogen particles continue to be found close to the l-band. At $24 \mathrm{~h}$ of recovery (C), more glycogen was observed and the individual glycogen particles are larger. The number of glycogen particles was not different than that seen $4 \mathrm{~h}$ post-exercise. Furthermore, the relative proportion of glycogen located in the intra-myofibrillar region was similar to that found at $4 \mathrm{~h}$. Finally, at the end of our observation period (D), significantly more glycogen was present compared to every other time recovery point. The increase in glycogen concentration from 24 to $48 \mathrm{~h}$ is produced by a non-significant increase in both the number and the size of individual glycogen particles. After $48 \mathrm{~h}$ of recovery, a significantly greater proportion of the myofibrillar glycogen was located within the myofibrils (intra-myofibrillar). The glycogen particles deposited in a row-like pattern along the actin and myosin myofilaments and the inter-myofibrillar glycogen was more generalised within that region. 


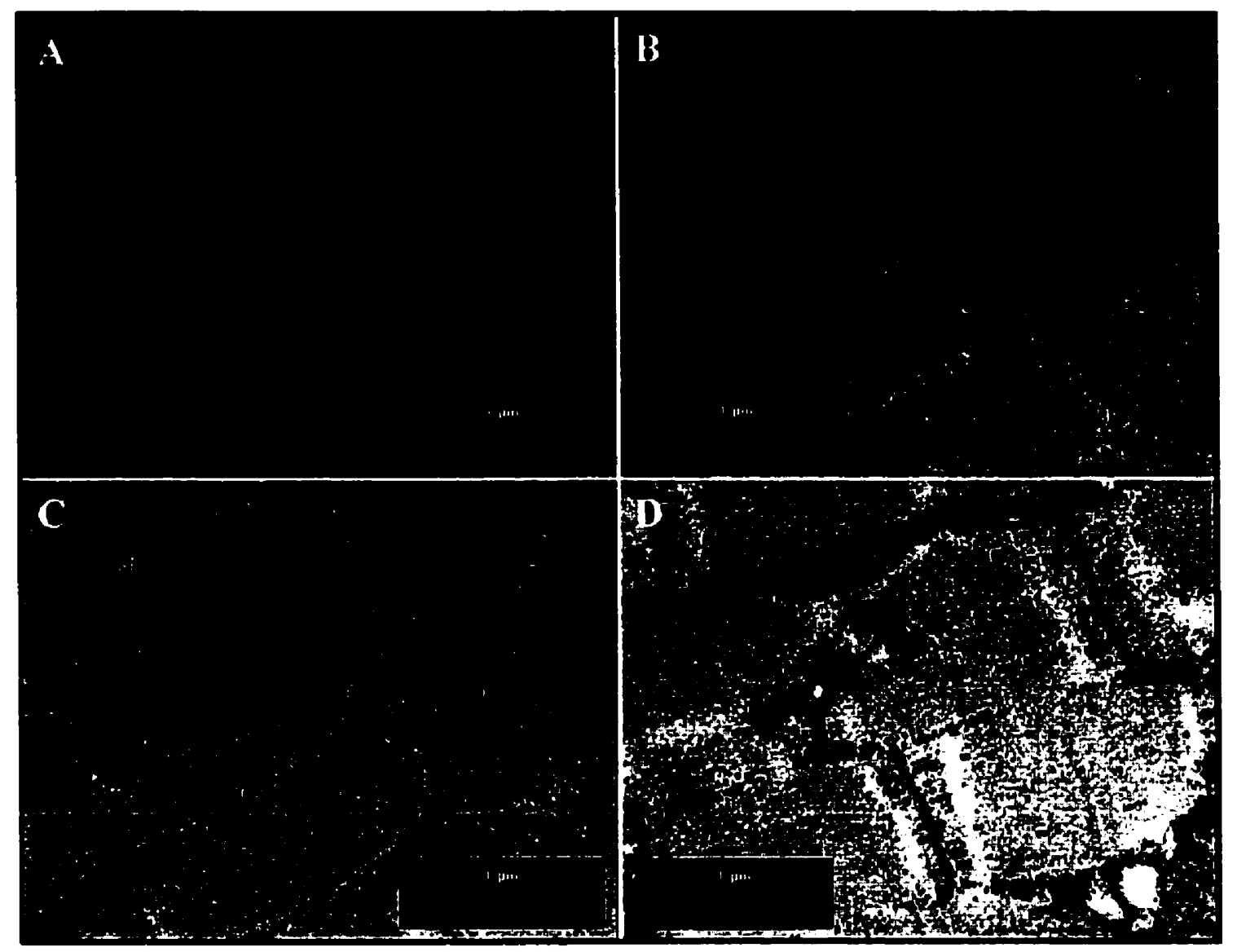

Figure 31. Illustration of muscle tissue from the same subject at $0 \mathrm{~h}(\mathrm{~A}), 4 \mathrm{~h}(\mathrm{~B}), 24 \mathrm{~h}$ (C), and $48 \mathrm{~h}(\mathrm{D})$ post-exhaustion. (20000 x) on a JEOL 1200 EX electron microscope.

\section{Discussion}

The purpose of the present study was to quantify the intra-cellular glycogen distribution in human skeletal muscles during the 48-hour recovery period from a moderate intensity, glycogen-depleting exercise. We hypothesised that exercise will lead to the preferential catabolism of myofibrillar over the subsarcolemmal glycogen. Furthermore, we intended to determine the post-exercise pattern of glycogen resynthesis with respect to glycogen particle size, number, and subcellular 
compartments. This is the first quantitative analysis of post-exercise intra-muscular glycogen distribution, with respect to particle sizes, numbers and subcellular locations. Furthermore, it represents the first report of the rates of glycogen synthesis in the different skeletal muscle subcellular locations. The major findings include: 1) this type of exercise led to the preferential depletion of intra-myofibrillar glycogen, 2) the increase in glycogen during the first four hours post-exercise was principally caused by an increase in the number of glycogen particles, while the glycogen increase over the next 40 hours of recovery was characterised by an increase in the volume of each individual particles, 3) a continuum in single particle diameters distribution was observed throughout the entire recovery period, 4) the net rate of glycogen synthesis in the first four hours postexercise was greater in the subsarcolemmal compared to the myofibrillar subcellular compartment, and 5) the net rate of single particle synthesis was greater in the myofibrillar compared to the subsarcolemmal subcellular compartment.

Time effect.

Depending on the time post-exercise, the increase in total glycogen concentration is characterised by fundamentally different changes (Figure 26). The initial $(0-4 h)$ increase in glycogen concentration appears to be mainly due to an increase in the number of glycogen particles $(p<0.05)$, with the increase in individual particle size contributing to a lesser extent $(p>0.05)$. On the other hand, from 4 to $24 \mathrm{~h}$ postexercise, the increase in glycogen content was entirely related to an increase in single particle size $(p<0.05)$, as the number of glycogen particles remained unchanged ( $>$ $0.05)$.

For the number of glycogen particles to increase, an increase in glycogenin, the protein backbone of glycogen particles, is required. Previous findings have demonstrated that, under most normal conditions, a deglucosylated form of glycogenin 
is not present in skeletal muscle (Smythe et al. 1990). If such is the case, it would suggest that glycogenin must be synthesised on demand. Kraniou and colleagues (2000) showed that only three hours following 60 minutes of moderate intensity exercise, skeletal muscle glycogenin mRNA increased more than two-fold. Furthermore, recent evidence by Shearer and co-workers (unpublished data) demonstrated that within 30 minutes of glycogen-depleting exercise, both glycogenin mRNA and in vitro activity (a reflection of protein amount and not in vivo activity) are increased. Taken together, these findings suggest that early in the recovery from a glycogen-depleting exercise, the primary mechanism responsible for increasing the intra-muscular concentration of glycogen was to increase the glycogenin expression / activity, thus leading to an increase in the number of glycogen particles. In agreement with this finding, glycogenin over-expression has been shown to lead to the production of more numerous, smaller, glycogen particles (Hansen et al. 1998; Skurat et al. 1997). Therefore, more particles being synthesised early in post-exercise, due to a greater glycogenin availability, would lead to a relatively slower increase in individual glycogen particle size, despite a high overall rate of glycogen synthesis.

In order for an increase in the size of individual glycogen particles to occur, both the availability of the substrate, UDP-glucose, and the activity of the rate-limiting enzyme, glycogen synthase (GS), must be increased. The mRNA and activity of GLUT4 and hexokinase, two of the major components regulating the availability of UDP glucose in skeletal muscles, have all been shown to increase within three hours postexercise (Koval et al. 1998; Kraniou et al. 2000; Kuo et al. 1999). In addition, muscle contraction has long been recognised to increase the GS activity (Connett and Sahlin 1996; Nielsen et al. 2001). Therefore, all the regulatory components necessary to cause an increase in the incorporation of UDP-glucose into glycogen particles, are present immediately post-exercise. However, despite this potential to expand the size of existing 
glycogen particles, we did not find a significant increase in the size of the individual particles during the first four-hour period post-exercise (Table 5). It is likely that a greater increase in the number of newly synthesised glycogen particles relative to the increase in the availability of UDP glucose is responsible for this finding immediately post-exercise.

The results from the present study suggest a biphasic pattern of glycogen synthesis, as both myofibrillar and subsarcolemmal net rates of synthesis were significantly greater during the $0-4 \mathrm{~h}$ period than the remainder of the recovery period (448 h) (Figure 27). Similarly, the Gt and PG rates of synthesis significantly decreased with time post-exercise, while MG synthesis rate did not change. The results of the biochemical analysis of glycogen are discussed elsewhere (Adamo et al. 1998b). These findings are in agreement with previous biochemical reports that have shown a rapid initial insulin-independent and a slower delayed insulin-dependent phase of glycogen synthesis (Price et al. 1994).

Price and colleagues $(2000)$ recently suggested the possibility that when glycogen concentration is decreased beyond a certain level, the glycogen particle is altered in such a way that the addition of glucose to existing particles is accelerated or that the rate of formation of new particle increases. Our results support the latter possibility, as the initial glycogen synthesis was shown to be mainly due to an increase in the number of glycogen particles. While speculative, there may be the existence of a link between the rapid insulin-independent and the slower insulin-dependent phase of glycogen synthesis, and the increase in glycogen particle number and size, respectively. For example, by an unknown mechanism, the glucose taken up during insulin stimulation could be preferentially directed to existing glycogen particles. 


\section{Subcellular locations.}

The results from the present study demonstrated that at every time point during a 48-hour recovery period from a glycogen-depleting exercise, the subsarcolemmal glycogen concentration was significantly greater than that in the myofibrillar location (Figure $29(A)$ ). This is in agreement with our observations in resting human skeletal muscles (Marchand et al. 2001). However, it should be emphasised that the myofibrillar space constitutes a much greater proportion of the total muscle tissue than does the subsarcolemmal space. Since the majority of the myofibrillar glycogen is located in the inter-myofibrillar space (Table 5), the inter-myofibrillar glycogen constitutes a much greater proportion of the absolute total skeletal muscle cell glycogen compared to both the intra-myofibrillar and the subsarcolemmal glycogen. This latter finding is in agreement with the only other studies to have addressed the compartmentalisation of skeletal muscle glycogen (Friden et al. 1985 and 1989; Sjostrom et al. 1982).

An important finding of the present study relates to the effect of time on the subsarcolemmal to total myofibrillar glycogen ratio (Table 5). Immediately post-exercise, the subsarcolemmal glycogen concentration was 3.3 times that of the myofibrillar glycogen concentration. This value decreased to 1.9 after four hours of recovery, and reached values of 1.5 and 1.6 at 24 and 48 hours, respectively. These later values are in agreement with those obtained in resting human skeletal muscles (Marchand et al. 2001). These findings suggest that the exercise caused a preferential depletion of the myofibrillar compared to the subsarcolemmal glycogen. Similarly, Friden and colleagues (1985) found the myofibrillar glycogen to be preferentially depleted compared to the subsarcolemmal glycogen during a marathon run. Furthermore, they report that the post-exercise inter-myofibrillar glycogen particles were decreased in diameter while the subsarcolemmal particles retained their size and shape. While their approach was semiquantitative and based on a small number of samples, our results substantiate and 
quantify the preferential depletion of myofibrillar over subsarcolemmal glycogen, although both fractions were used to a significant extent during this specific exhaustive type of exercise.

Previous studies have found both the intra- and inter-myofibrillar glycogen to be predominately associated with the myofibrillar I band region (Friden et al. 1985 and 1989). The present study confirms the biased I-band location for both the inter-and intra-myofibrillar glycogen. With increasing total glycogen concentration following the glycogen-depleting exercise, both the intra- and inter-myofibrillar glycogen appear to spread longitudinally within their respective compartments, away from the I-band, hence giving rise to a more longitudinally uniform myofibrillar glycogen distributions (Figure 31). It is interesting to relate these latter findings with the results of a recent study by Nielsen and co-workers (2001). They examined the role of glycogen concentration in regulating the subcellular distribution of GS. Their results obtained by confocal microscopy showed the antibody against GS to be preferentially located at the level of the I band when glycogen concentrations are low, and to be spread longitudinally throughout the muscle fibre when glycogen concentration are elevated. These results are in agreement with the subcellular distribution of glycogen particles shown in the present study.

Friden and co-workers (1989) suggested that this inter-myofibrillar I band bias of skeletal muscle glycogen could be related to the fact that the I band represents the portion of the sarcomeres with the highest sarcoplasmic reticulum (SR) density. In fact, the physical association of the SR with the glycogen-enzyme complex (glycosomes) has been consistently demonstrated (Goldstein et al. 1985; Meyer et al. 1970; Rybicka $1981 \mathrm{a}$ and 1981b; Wanson and Drochmans 1968 and 1972). While the exact physiological meaning of these protein interactions remains to be clarified, the interaction between glycosomes and SR, in close proximity to the transverse tubular 
system where the GLUT-4 are known to translocate in response to insulin (Lund et al. 1993) and exercise (Cartee et al. 1989), could facilitate the synthesis of glycogen by increasing glucose availability.

As described in the methods section, the myofibrillar glycogen represents the sum of intra- and inter-myofibrillar glycogen. Immediately post-exercise, only $3 \%$ of the myofibrillar glycogen was located in the intra-myofibrillar space. Four hours later, the proportion was increased to $12 \%$, and remained unchanged for the remainder of the recovery period. These data suggest that the proportion of intra-myofibrillar glycogen used during this moderate intensity glycogen-depleting exercise was greater than that of inter-myofibrillar glycogen. Given the close proximity of the intra-myofibrillar glycogen to the contractile apparatus, this finding is not surprising. While a greater proportion of intra-myofibrillar glycogen was used, in absolute terms this glycogen only contributes to a small proportion of the total glycogen used during exercise.

A ratio of intra-myofibrillar to myofibrillar glycogen of $12 \%$ corresponds to the value obtained previously in resting human skeletal muscles (Marchand et al. 2001). Hence, while the glycogen-depleting exercise led to the relative depletion of intramyofibrillar glycogen, the resynthesis was such that the normal resting ratio of intramyofibrillar to myofibrillar glycogen was re-established only four hours following the completion of the glycogen-depleting exercise. Therefore, these data suggest that the synthesis of intra-myofibrillar glycogen was prioritised over that of the inter-myofibrillar glycogen immediately following glycogen depletion exercise. From a teleological point of view, this could represent a mechanism to ascertain the availability of energy to the contractile apparatus in the eventuality of an emergency situation requiring an intense muscle contraction. Interestingly, Nielsen and colleagues (2001) recently provided information to suggest that GS translocates from a glycogen-enriched membrane 
fraction (representing both subsarcolemmal and inter-myofibrillar subcellular locations) to a cytoskeleton fraction (intra-myofibrillar) when glycogen concentration was decreased. These data provide further evidence to suggest that glycogen synthesis is prioritised in the intra-myofibrillar subcellular location over both subsarcolemmal and inter-myofibrillar locations.

The results of the present study also demonstrated that, while the glycogen volume is more concentrated in the subsarcolemmal than the myofibrillar space, the glycogen particles are smaller in the subsarcolemmal space (Figure 29 (B)). The difference was only significant at 24 and 48 hours post-exercise, which correspond to the recovery time when resting levels of glycogen were reached. This is in agreement with a previous study performed in resting human skeletal muscles (Marchand et al. 2001). At any given recovery time point, the subsarcolemmal glycogen was made of 1.7 to 2.9 times more glycogen particles than was the myofibrillar space (Table 5). Therefore, the subsarcolemmal and the myofibrillar fractions of glycogen appear to have their glycogen present under two different patterns, the former having more numerous, smaller glycogen particles, and the latter less numerous, larger particles. The physiological meaning of this finding is unclear.

It has been previously suggested that the subsarcolemmal glycogen particles could serve to ensure adequate energy store for the maintenance of the plasma membrane potential and / or to act as an intra-cellular reserve energy supply (Friden et al. 1985 and 1989). In resting conditions, the main way for glucose to enter the skeletal muscle cells is through GLUT-1, which are exclusively located at the sarcolemmal membrane. Our finding of smaller, more numerous glycogen particles in the subsarcolemmal space, despite a greater glycogen concentration, may indicate an 
increased glycogen turnover in that compartment compared to the myofibrillar glycogen, which could be associated with a constant energy demand.

This study is the first to report the rates of glycogen synthesis in the different skeletal muscle subcellular locations. While the net rates of glycogen synthesis decreased with time post-exercise in both subsarcolemmal and myofibrillar space, the net rate of glycogen synthesis was significantly greater in the subsarcolemmal than myofibrillar compartment from 0 to $4 \mathrm{~h}$ post-exercise (Figure 27).

While the mechanism responsible for this finding is unclear, a different pattern of glucose transport between subcellular locations could partly explain the difference. In skeletal muscles, the GLUT-1 transporter is restricted to the cell surface, while the more abundant GLUT-4 transporter is largely sequestered intracellularly and it is rapidly translocated to the cell surface in response to insulin, exercise, or hypoxia (Klip et al. 1996). Furthermore, the translocation of GLUT-4 has been reported to occur mainly at the level of the transverse tubular system (Zierler 1999). Hence, a greater rate of subsarcolemmal glycogen synthesis early in exercise could be related to the presence of a faster glucose transporter system in that location. Indeed, the subsarcolemmal space would receive glucose supply from the sarcolemmal GLUT-1, as well as from the GLUT4 associated to the transverse tubular system of the first myofibril.

Another possibility relates to the smaller size of the particles in the subsarcolemmal compared to the myofibrillar locations (Figure 29 (B)). It has been suggested that the $K_{m}$ for glucose is smaller for the GS associated with PG than for the GS associated with MG (Curtino et al. 2000; Lomako et al. 1993). We (Marchand et al. 2001) and others (Skurat et al. 1997) have provided evidence against the previous suggestion that PG represents a stable intermediate in glycogen biosynthesis (Alonso et al. 1995). We believe that PG simply represents a glycogen species of smailer 
molecular weight than the MG species, within a continuum range of particle size. Taken together, these findings could suggest that the GS associated with a smaller glycogen species would be more active than that of a larger one.

While different forms of GS have been proposed, it is more likely that as the particle size increases post-exercise, the relationship between carbohydrate, GS and the regulatory enzymes (i.e. PP1 and the kinases) within the glycogen particle is altered, or that the increasing particle density impedes on the ability of GS to add UDP-glucose to the nonreducing ends of the glycosomes. This could explain a greater net rate of glycogen synthesis in the subsarcolemmal space, where the glycogen particles are relatively smaller, and hence, GS is more active.

Surprisingly, despite the greater net rate of glycogen synthesis in the subsarcolemmal space during early recovery, the net rate of single particle synthesis was greater in both myofibrillar spaces than in the subsarcolemmal one (Figure 30). This dissociation between the net rates of glycogen and individual particle synthesis during the early post-exercise period implies that, relative to the myofibrillar space, the subsarcolemmal space increases its glycogen concentration by making new granules instead of making the existing ones bigger. This is supported by the fact that from 0 to 4 hours, the absolute increase in number of glycogen particles per $\mu \mathrm{m}^{3}$ of tissues in the subsarcolemmal space was 1.7 times that of the myofibrillar space (Table 5).

One would expect that the subcellular compartment with the highest net rate of glycogen synthesis would also possess the highest net rate of individual particle synthesis. The dissociation between the net rate of glycogen synthesis and the net rate of individual particle synthesis actually suggests that it is the net rate of individual particle synthesis, and not the net rate of overall glycogen synthesis, that is dependent on the glycogen concentration. Numerous investigations have demonstrated the overall tissue net glycogen synthesis rate to be dependent on the overall tissue glycogen 
concentration (Laurent et al. 2000; Montell et al. 1999; Nielsen et al. 2001; Price et al. 2000). The greater subsarcolemmal glycogen synthesis rate, despite a greater glycogen concentration in that space, disagrees with this concept. However, our analyses of individual particle synthesis rates suggest that the well-known inverse relationship between the rate of glycogen synthesis and glycogen concentration is operational at the single particle level. Therefore, the size of individual particle would alter the shape of the particle, and consequently its interactions with the metabolising enzymes, and hence regulate the net rate of glycogen synthesis.

Single particle distribution.

We previously demonstrated a continuum in the distribution of glycogen particle diameters in resting human muscle (Marchand et al. 2001). This study demonstrated for the first time that a continuum in single particle diameter distribution is observed throughout a range from low to normal-high glycogen concentration (Figure 28). The range of particle diameter observed in the present study ( 7 to $43 \mathrm{~nm}$ ) is in agreement with the majority of the literature, in which glycogen $\beta$ particles ranging between 15 and $40 \mathrm{~nm}$ in diameter have been reported for resting human and animal tissues, including skeletal muscles (Biava 1963; Revel 1964; Rybicka 1996). While most of these studies provided only gross estimates of $\beta$ particles diameter, very few actually examined the distribution of $\beta$ particle diameter (Friden et al. 1989; Scott and Still 1968; Wanson and Drochmans 1968). When the results from sub-optimal tissue preparation are excluded (Rybicka 1996), these studies provide evidence to suggest that the diameter of glycogen $\beta$ particles is normally distributed between 15 and $40 \mathrm{~nm}$. Taken together, our results confirm those of previous TEM-based experiments and further suggest that the distribution of skeletal muscle glycogen particles represents a continuum of size at any 
given total skeletal muscle glycogen concentrations.

Two independent sets of evidence have previously suggested that PG, the low molecular mass form of glycogen, serves as a stable intermediate in the pathways to and from depot glycogen (MG) (Alonso et al. 1995; Friden et al. 1989). However, other biochemical studies have challenged the role PG as a stable intermediate, and demonstrated a continuous range of glycogen particle species, in which the distribution was found to move towards smaller or larger particle sizes under different conditions (Roach and Skurat 1997). The present findings, which are based on the analysis of more than 180000 individual glycogen particles, as well as those of other TEM based studies, provide direct evidence to support this conclusion. Indeed, in the present study, despite glycogen depletion to levels as low as $36 \mathrm{mmol} / \mathrm{kg}^{-1} \mathrm{dw}$, a continuous range of glycogen particles was seen. As total muscle glycogen concentration increased, the frequency distribution of glycogen particle sizes shifted to the right, giving rise to a normal distribution of larger particles (Figure 28).

The structure of glycogen particles is important in understanding the significance of the present data. According to the accepted Whelan's model of glycogen structure, the glycogen particle is a spherical unit arranged in concentric layers or tiers (Goldsmith et al. 1982). Due to its particular branching pattern, the amount of glucose directly available to be released by glycogen phosphorylase (GP) has been reported to be equal to $-35 \%$ of the total glucose contained in that particle, independent of the number of tiers of the particle (Meléndez-Hevia et al. 1993). It is generally accepted that size limit is determined by the density of the last $\left(12^{\text {th }}\right)$ tier (Goldsmith et al. 1982). According to the calculated thickness of a single tier $(3.8 \mathrm{~nm})$, a maximal size of $42 \mathrm{~nm}$ has been predicted for a $\beta$ particle (Goldsmith et al. 1982). It is striking how this value corresponds to the measured single particle diameter in the present study, as well as 
others (Biava 1963; Marchand et al. 2001; Revel 1964). Hence, in a mature $\beta$-particle of $42 \mathrm{~nm}$ containing a total 55000 glucose residues (Goldsmith et al. 1982), one can predict that approximately $19000(-35 \%$ of 55000$)$ will be present in the $12^{\text {th }}$ tier, 12400 $(\sim 23 \%$ of 55000$)$ in the $11^{\text {th }}$ tier, $8100(\sim 15 \%$ of 55000$)$ in the $10^{\text {th }}$ tier, and $5300(\sim-10$ $\%$ of 55000 ) in the $9^{\text {th }}$ tier, etc. Therefore, only approximately $18 \%$ of the total glucose of a mature $\beta$-particle is located in tiers 8 to 1 . Accordingly, it is clear that an increase in individual particle diameter will contribute to a much greater increase in total muscle glucosyl units (glycogen concentration) when the particle is within the greater size range. Thus, the increase in particle diameter from 13 to $16 \mathrm{~nm}$ (the mean values for 0 to $4 \mathrm{~h}$ ), produces an increase in glycogen concentration that is much smaller compared to that produced by an increase in particle diameter from 23 to $24 \mathrm{~nm}$ (the mean values for 24 to $48 \mathrm{~h}$ ). Hence, while not statistically significant, the small changes in diameter later in recovery are likely to be physiologically significant.

This also directly relates to the subcellular location effect on single particle volume illustrated in Figure 29 (B). For the first 4 hour recovery, there was no statistical difference in particle volume between subcellular locations. Thereafter however, the subsarcolemmal glycogen particles were shown to be significantly smaller than both the superficial and deep myofibrillar particles. Despite a $10 \%$ difference in particle volume between the superficial and deep myofibrils at $48 \mathrm{~h}$ post-exercise, which corresponds to a $0.7 \mathrm{~nm}$ in diameter, the difference was not shown to be statistically significant. If one considers that, in those higher ranges of particle size, a relatively small increase in size may in fact represent a large increase in glucose content, the difference might indeed be very significant metabolically. Hence, it is possible that an important difference actually exists between the deep and superficial subcellular locations in terms of glucose content of individual glycogen particles, but our method does not allow its detection. 
Our inability to detect the increasing density in glucosyl units with increasing particle size represents a weakness of the present technique. However, given the strong correlation between the single particle volume and the biochemically determined glycogen concentration $\left(r^{2}=0.96\right)$, we conclude that the size of the glycogen particle is a valid indicator of total muscle glycogen concentration (refer to Chapter IV - Figure 20).

Fibre types.

Numerous investigations have demonstrated that the extent of glycogen depletion in specific fibre types is dependent on exercise intensity (Blom et al 1986; Essen 1978; Vollestad et al. 1985). The results presented in the current study are in agreement with these findings. We found that immediately post-exercise, the myofibrillar glycogen concentration in type I fibres was 3 and 25 times less than in type IIA and IIB fibres, respectively (both $p<0.05$ ). After four hours of recovery, the myofibrillar glycogen concentration was no longer different between fibre types. Furthermore, while this was not significant, a similar trend was observed for the glycogen in the subsarcolemmal space.

Throughout the entire recovery period, the single particle volume was significantly greater in type IIB compared to either type IIA and I fibres. These findings are in agreement with a previous experiment performed in samples obtained from 11 resting human skeletal muscle (Marchand et al. 2001). In that study, the mean particle size of type I fibres was found to be significantly smaller than both type IIA and IIB fibres. As discussed previously (Marchand et al. 2001), we speculate that the potential physiological explanation for this fibre type difference could be linked to the fibre type differences in glycogenin availability (Hansen et al. 2000), or alternatively, to the more constant glycogen turnover in type I and IIA compared to IIB fibres. 
Fibre type was also found to influence the relative proportion of glycogen located in the intra- and inter-myofibrillar region (Table 5). From 4 hours post-exercise, the relative proportion of the myofibrillar glycogen located in the intra-myofibrillar space (intra / myofib) was found to be greater in type I than both type II fibres. However, this fibre type effect was only significant $(p<0.004)$ when time $0 \mathrm{~h}$ was excluded from the analysis. We believe that this analysis is meaningful considering that the exercise caused selective glycogen depletion in type I fibres. In addition, the exercise led to a preferential decrease in intra- relative to inter-myofibrillar glycogen. To test this, we used the 48-hour value as baseline and calculated the $\%$ of decrease in glycogen volume immediately post-exercise (data not shown). The results showed that $98 \pm 1$ and $92 \pm 2 \%$ of the glycogen in the intra- and inter-myofibrillar locations were used, respectively $(P=0.03)$. We are aware that the effect of exercise on the subcellular location of glycogen could have altered the 48-hour values compared to resting samples. However, the proportion of myofibrillar glycogen located in the intramyofibrillar region obtained at 48 hours post-exercise in the present study is similar to that obtained previously in resting human subjects (Marchand et al. 2001).

Furthermore, immediately post-exercise, the intra / myofib was much lower in the fibre type preferentially used during exercise (i.e. type I), and increased with time postexercise to levels actually higher in type I compared to the type II fibres. Together, these findings support the notion that continuous moderate intensity cycling to exhaustion leads to a preferential depletion of the myofibrillar glycogen located in the intra-compared to the inter-myofibrillar region. However, as pointed out earlier, in absolute terms, the total muscle amount of glycogen located in the inter-myofibrillar space was significantly greater than that in the intra-myofibrillar space; therefore, its contribution to the absolute amount of muscle myofibrillar glycogen used during this type 
of exercise is likely to be much greater.

\section{Conclusion}

This study used a recently developed method for quantifying the subcellular distribution of skeletal muscle glycogen to characterise the pattern of glycogen repletion following a glycogen-depleting exercise. We confirmed our hypothesis that prolonged moderate intensity exercise leads to the preferential depletion of myofibrillar over the subsarcolemmal glycogen, and that the pattern of glycogen synthesis differed between subcellular compartments. Following cycling at $70 \% \mathrm{VO}_{2} \max$ to exhaustion, during which the intra-myofibrillar glycogen was preferentially depleted: 1) the initial increase in glycogen was principally due to an increase in the number of glycogen particles, while an increase in single particle size was responsible for the increase in glycogen later in recovery, 2) a continuum in single particle diameter distribution was observed throughout the entire recovery period, 3) the net rate of glycogen synthesis in the first four hours post-exercise was greater in the subsarcolemmal compared to the myofibrillar subcellular compartment, and 4) the net rate of individual particle synthesis was greater in the myofibrillar compared to the subsarcolemmal subcellular compartment. By showing that the diameters of glycogen particles present as a continuum of sizes, the current findings provide evidence against the point of view that PG is a stable intermediate in glycogen synthesis. Furthermore, these findings suggest that the wellknown effect of glycogen concentration on the net glycogen synthesis is regulated at the local glycosome level and is based on the size of individual particles. 


\section{Chapter VII}

Experiment 3: Quantitative analysis of the changes in glycogen particle size and number in different subcellular locations in human skeletal muscle during high-intensity exercise. 


\section{Introduction}

While skeletal muscle glycogen and its metabolism have been studied extensively using standard biochemical analysis, the information provided by direct visualisation of glycogen within skeletal muscle using transmission electron microscopy (TEM) is limited to a few qualitative investigations. We recently developed a TEM-based method for quantifying the subcellular distribution of skeletal muscle glycogen, and employed it to characterise the glycogen distribution in resting skeletal muscle samples (Marchand et al. 2001). The results confirmed earlier observations that resting human skeletal muscle glycogen distribution is compartmentalised and heterogeneous with respect of its pattern of distribution (Friden et al. 1985 and 1989; Sjostrom et al. 1982a). In agreement with the available literature (Biava 1963; Revel 1964; Rybicka 1996), we also demonstrated that single glycogen particles ( $\beta$ particles) range from 10 to $44 \mathrm{~nm}$ ( $25 \pm 3 \mathrm{~nm}$ ) and that they follow a normal distribution. Finally, we found significant differences in the subcellular distribution of glycogen between subcellular locations and fibre types.

Using the same TEM-based technique, we subsequently examined the pattern of glycogen repletion during 48 hours of recovery following moderate intensity, glycogendepleting exercise (Chapter Vl). The results showed that there are compartmentspecific differences in glycogen resynthesis. Furthermore, we found that the characteristics of the resynthesis of glycogen post-exercise differed according to the time post-exercise. Specifically, we found the increase in glycogen during the first four hours post-exercise to be caused by an increase in the number of glycogen particles and the glycogen increase in the remainder of recovery to be characterised by an increase in the volume of individual particles. Finally, we found a dissociation between the influence of glycogen concentration on the rate of glycogen synthesis at the total 
muscle level and that at the level of the individual glycogen particles. These results were taken to suggest that the well-known effect of glycogen concentration in influencing net rate of glycogen synthesis is exerted at the local glycosome level, and that it is based on the size of individual particles.

The impact of different types of exercise on the subcellular location of glycogen has been studied by Ekblom and co-workers (Friden et al. 1985 and 1989; Sjostrom et al. 1982). Their results suggested that the glycogen in different subcellular locations is catabolized differently according to the type of exercise performed. Indeed, they showed that prolonged moderate intensity exercise leads to the preferential depletion of the myofibrillar glycogen, whereas intense exercise leads to the preferential degradation of the subsarcolemmal and intra-myofibrillar glycogen. However, these findings must be interpreted with caution, since they are based on qualitative observations.

Nevertheless, their findings are compatible with the emerging concept of the importance of subcellular location as a vital control mechanism in glycogen metabolism. Accordingly, independent investigations have demonstrated that the intracellular translocation of glycogen synthase (Nielson et al. 2001), GLUT-4 (Cartee et al. 1989; Lund 1993) and glucokinase (Brown 1997) are important in controlling glucose disposal and glycogen metabolism. The direct visualisation of some of the essential components of glycogen metabolism provided by TEM-based analysis allows for the study of subcellular compartmentalisation as a supplemental mechanism for controlling glycogen metabolism. The purpose of the present study was therefore to provide a quantitative analysis of the distribution and pattern of glycogen degradation during intense exercise. We hypothesised that both myofibrillar and subsarcolemmal glycogen would be significantly degraded during the exercise. Furthermore, we hypothesised that a 
decrease in both size and number of glycogen particles would contribute to this decrease in glycogen concentration.

\section{Methods}

Subjects. The study was approved by the University of Guelph's Human Ethics Committee and conformed to the standards set by the Declaration of Helsinki. Six male subjects were informed in writing about the nature of the experiment, volunteered to participate, and signed a consent form. The mean ( \pm S.E.M.) age, height, weight, and $\mathrm{VO}_{2} \mathrm{max}$ of the subjects were $26 \pm 1 \mathrm{yr}, 183 \pm 4 \mathrm{~cm}, 91 \pm 3 \mathrm{~kg}$, and $50 \pm 2 \mathrm{~mL} \cdot \mathrm{kg}^{-1}$. $\min ^{-1}$, respectively.

Pre-trial procedures. The subjects initially underwent an incremental $\mathrm{VO}_{2}$ max test on an electronically braked cycle ergometer (Quinton Excaliber). A week before the trial, a practice ride was completed. This ride served two purposes: (1) confirmation of the exercise workload intensity and (2) familiarisation of the subjects to the workload and experimental protocol.

Experimental protocol. Subjects arrived at the laboratory after consuming a lowcarbohydrate bieakfast at least three hours before testing. Under local anaesthesia, an incision was made over the vastus lateralis. Resting muscle biopsies were obtained from the vastus lateralis by use of the percutaneous needle biopsy technique (Hultman 1967) with suction modification (Evans 1982). The subjects then underwent two 90second bouts of exercise at $130 \% \mathrm{VO}_{2}$ max on a cycle ergometer, separated by a 10 minute rest period. A second muscle biopsy was obtained immediately following the completion of the second exercise bout. 
Analyses, calculations and statistics.

Please refer to Chapter IV - Detailed Methods.

\section{Results}

Biochemical data.

The subjects had a pre- and post-exercise average ( \pm S.E.M.) Gt of $437 \pm 62$ and $223 \pm 21$ glucosyl units $\cdot \mathrm{kg}^{-1} \mathrm{dw}$, respectively. The pre- and post-exercise $\%$ of $\mathrm{Gt}$ in the form of $P G$ averaged $74 \pm 4$ and $77 \pm 6$, respectively.

TEM data.

Effect of exercise.

The pre- and post-exercise values for all dependent variables are presented in Table 6. The values represent the weighted means, which were obtained from all three fibre types (see methods) and used as a total muscle value. The exercise caused a significant decrease in biochemical Gt, myofibrillar glycogen volume, as well as in the relative proportion of myofibrillar glycogen located in the intra-myofibrillar region (intra / myofib). Surprisingly, the individual particle size and the subsarcolemmal glycogen volume were not significantly affected by the exercise. Furthermore, although a trend was present in both compartments $(P=0.11)$, the number of glycogen particles in myofibrillar and subsarcolemmal locations were not significantly affected by the exercise. Herice, the decrease in myofibrillar glycogen volume was achieved by a non-significant decrease in particle number $(P=0.11)$ and volume $(P=0.50)$. 
Table 6. Summary of the pre-and post-exercise values for all dependent variables.

\begin{tabular}{|c|c|c|c|c|c|c|c|c|c|c|}
\hline & $\begin{array}{l}\text { Blochemical } \\
\text { totol glycogen } \\
\text { (mmol / igg dw) }\end{array}$ & XPG & $\begin{array}{l}\text { Partele } \\
\text { diameter } \\
\text { (nm) }\end{array}$ & $\begin{array}{l}\text { Particle } \\
\text { Volume } \\
\left(u m^{2} \times 10^{3}\right)\end{array}$ & $\begin{array}{c}\text { Myoribrillar } \\
\text { particles } \\
\text { (per um') }\end{array}$ & $\begin{array}{l}\text { Myofibrillar } \\
\text { olycogen volume } \\
\left(4 \mathrm{~m}^{3} \times 10^{4}\right)\end{array}$ & $\begin{array}{c}\text { Intra I myont } \\
\text { (\%) }\end{array}$ & $\begin{array}{c}\text { Subsarcolemmal } \\
\text { (particles } \\
\text { (per um') }\end{array}$ & $\begin{array}{l}\text { Subsarcolenmal } \\
\text { glycogen volume } \\
\left(4 \mathrm{~m}^{3} \times 10^{4}\right)\end{array}$ & $\begin{array}{l}\text { Subsar } \\
\text { Imyoftb } \\
\text { (unita) }\end{array}$ \\
\hline $\begin{array}{l}\text { Pre-exarcise } \\
\text { Mean } \\
\text { S.E.M. }\end{array}$ & $\begin{array}{l}437 \\
62\end{array}$ & $\begin{array}{c}74 \\
4\end{array}$ & $\begin{array}{c}284 \\
1.1\end{array}$ & $\begin{array}{l}99 \\
11\end{array}$ & $\begin{array}{c}2982 \\
423\end{array}$ & $\begin{array}{c}278 \\
26\end{array}$ & $\begin{array}{c}132 \\
1.0\end{array}$ & $\begin{array}{c}5943 \\
884\end{array}$ & $\begin{array}{l}452 \\
62\end{array}$ & $\begin{array}{l}167 \\
0.22\end{array}$ \\
\hline & $P=00 t$ & $P=029$ & $P=061$ & $P=0.50$ & $P=0.11$ & $P=0.0038$ & $P=0.0028$ & $P=0.11$ & $P=0.16$ & $P=068$ \\
\hline $\begin{array}{l}\text { Post-axercise } \\
\text { Moan } \\
\text { S.E.M. }\end{array}$ & $\begin{array}{c}223 \\
21\end{array}$ & $\begin{array}{c}77 \\
6\end{array}$ & $\begin{array}{c}258 \\
06\end{array}$ & $9:$ & $\begin{array}{r}2084 \\
187\end{array}$ & $\begin{array}{c}192 \\
23\end{array}$ & $\begin{array}{c}10.0 \\
13\end{array}$ & $\begin{array}{c}4248 \\
570\end{array}$ & $\begin{array}{c}339 \\
45\end{array}$ & $\begin{array}{l}178 \\
0.16\end{array}$ \\
\hline
\end{tabular}

Values are weighted means from all three fibre types (see methods) and represent a total muscle value. The "P" values represent the results from the statistical analysis comparing the pre- and postexercise values. \% PG is the percentage of total biochemical glycogen in the pro-glycogen form. The "particle diameter" and "particle volumes" represent the total fibre average. The myofibrillar and subsarcolemmal glycogen volumes represent glycogen concentrations as measured by TEM-based analysis. "Intra / myofib" is the proportion of myofibrillar glycogen located in the intra-myofibrillar region. The "Subsar / Tmyofib" is the ratio of the subsarcolemmal over myofibrillar glycogen volumes.

Figure 32 illustrates the pre- and post-exercise glycogen volumes, as well as the percentage of pre-exercise glycogen volume used during the exercise, in the different subcellular compartments. The biochemical glycogen values are also reported. The percentage of pre-exercise glycogen used during the exercise was significantly different between subcellular compartments $(P=0.024)$. While an average of $53 \%$ of preexercise intra-myofibrillar glycogen volume was used, the corresponding values for the inter-myofibrillar and subsarcolemmal glycogen were 30 and $25 \%$, respectively. Similarly 46,44 and $58 \%$ of the biochemically determined Gt, PG and MG, respectively, was used during the exercise. In contrast to the significant difference observed between different subcellular compartments, the percentages of decrease in biochemically determined glycogen were not significantly different between $G$, $P G$ and $M G(P=0.33)$. 


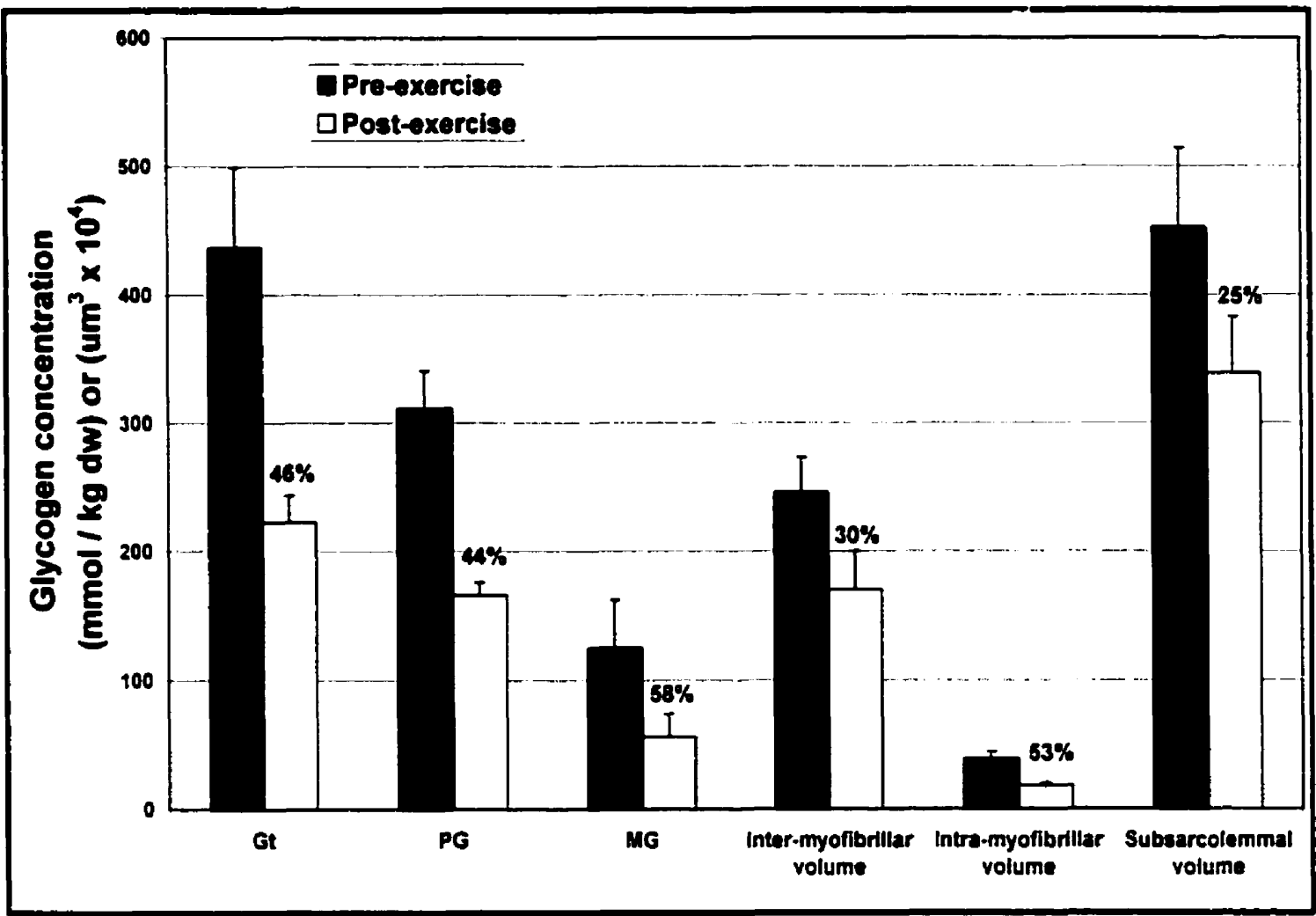

Figure 32. Effect of exercise on glycogen concentrations. The pre- and post-exercise glycogen volumes are presented for different subcellular compartments. The percentage $(\%)$ is the $\%$ decrease from pre-exercise value. The corresponding values for biochemically determined total (Gt), pro- (PG) and macro- (MG) glycogen are also reported. The \% of pre-exercise glycogen used during exercise were significantly different between subcellular compartments $(P=0.024)$. In contrast, the percentages of decrease in biochemically determined glycogen were not significantly different between $G t, P G$ and $M G(P=0.33)$.

Effect of subcellular locations.

The effect of subcellular location on different glycogen parameters is reported in Table 7. As noted previously (Marchand et al. 2001), particle diameter and volume, number of glycogen particles and glycogen volumes were all affected by subcellular location ( $P<0.0001)$. Both pre- and post-exercise, the glycogen particles were smaller 
in the subsarcolemmal compared to the myofibrillar locations (both superficial and deep myofibrils). In contrast, the glycogen volume and the number of glycogen particles were greater in the subsarcolemmal space than in either the superficial or the deep myofibrils. No significant difference was present between the deep and superficial myofibrils for any of the dependent variables. The effect of exercise on glycogen concentration (volume) was significant in both deep and superficial myofibrils, but not in the subsarcolemmal space $(P=0.16)$. In addition, a trend for the number of glycogen particles to decrease from pre- to post-exercise was seen in all locations $(P=0.11)$. No significant time $x$ subcellular location interactions were detected ( $P$ values not shown).

Table 7. Summary of the effect of subcellular location for all dependent variables.

\begin{tabular}{|c|c|c|c|c|c|c|}
\hline \multicolumn{3}{|c|}{ Subloc } & $\begin{array}{l}\text { Particle diamotor } \\
\text { (nm) }\end{array}$ & $\begin{array}{l}\text { Particle volume } \\
\left(4 \mathrm{~m}^{3} \times 10^{7}\right)\end{array}$ & $\begin{array}{c}\text { Number of particles } \\
\text { (per um })\end{array}$ & $\begin{array}{c}\text { Glycogen volume } \\
\left(\mathrm{um}^{3} \times 10^{4}\right)\end{array}$ \\
\hline \multicolumn{3}{|c|}{ Main effect of time } & N.S. & N.S. & $P=0.1101$ & $P=0.0038$ \\
\hline \multicolumn{3}{|c|}{ Main effect of subcellular locations } & $P<0.0001$ & $P<0.0001$ & $P<0.0001$ & $P<0.0001$ \\
\hline \multirow[t]{2}{*}{ Pre-exercise } & $\begin{array}{l}\text { Subsarcolemmal } \\
\text { Superficial }\end{array}$ & $\begin{array}{l}\text { Mean } \\
\text { SEM } \\
\text { Mean } \\
\text { SEM }\end{array}$ & $\begin{array}{c}25.0 \\
0.6 \\
(a b) \\
26.4 \\
0.5\end{array}$ & $\begin{array}{c}85 \\
5 \\
(a b) \\
99 \\
6\end{array}$ & $\begin{array}{c}6112 \\
509 \\
(a b) \\
2970 \\
282\end{array}$ & $\begin{array}{c}479 \\
36 \\
(a b) \\
261 \\
12\end{array}$ \\
\hline & Deep & $\begin{array}{l}\text { Mean } \\
\text { SEM }\end{array}$ & $\begin{array}{l}26.7 \\
0.6\end{array}$ & $\begin{array}{c}103 \\
6\end{array}$ & $\begin{array}{l}2993 \\
223\end{array}$ & $\begin{array}{c}287 \\
18\end{array}$ \\
\hline \multirow[t]{2}{*}{ Post-exercise } & Subsarcolemmal & $\begin{array}{l}\text { Mean } \\
\text { SEM } \\
\text { Mean } \\
\text { SEM }\end{array}$ & $\begin{array}{c}24.5 \\
0.3 \\
(a b) \\
25.9 \\
0.3\end{array}$ & $\begin{array}{c}78 \\
2 \\
(a b) \\
92 \\
3\end{array}$ & $\begin{array}{c}4473 \\
333 \\
(b b) \\
2112 \\
140\end{array}$ & $\begin{array}{c}348 \\
28 \\
(a b) \\
195 \\
15\end{array}$ \\
\hline & Deep & $\begin{array}{l}\text { Mean } \\
\text { SEM }\end{array}$ & $\begin{array}{c}26.4 \\
0.4\end{array}$ & $\begin{array}{c}98 \\
4\end{array}$ & $\begin{array}{c}2153 \\
127\end{array}$ & $\begin{array}{c}210 \\
17\end{array}$ \\
\hline
\end{tabular}

a : significant difference compared to superficial myofibrils $(p<0.05)$. b: significant difference compared to deep myofibrils $(p<0.05)$. "N.S." means non-significant.

The effect of subcellular location on single particle diameter is illustrated in Figure 33. The pre- and post-exercise results are presented for each subcellular 
location. Both pre- $(A)$ and post- $(B)$ exercise, the particle diameters were distributed normally within each subcellular location. However, the frequency distribution of single particles located in the subsarcolemmal space is shifted to the right compared to that of both deep and superficial myofibrillar locations. This is seen both pre- and postexercise. The mean single particle diameter values for each subcellular location are presented in Table 7.

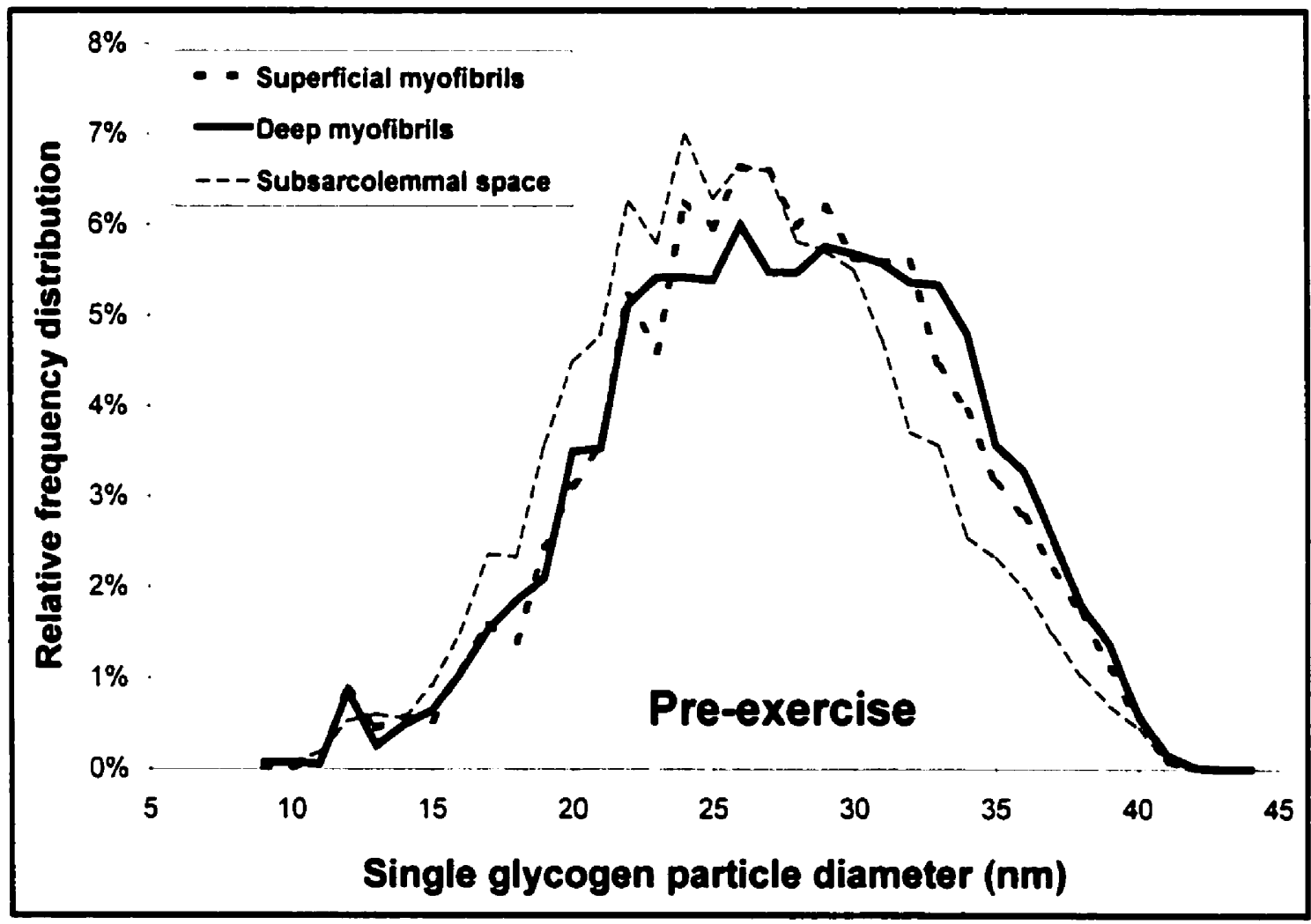

Figure 33 (A). Pre-exercise relative frequency distribution of single particle diameter $(\mathrm{nm})$ in the three subcellular locations (subsarcolemmal space, superficial and deep myofibrils), across fibre type. The relative frequency distribution expresses the frequency as a percentage of the absolute frequency. The data from all six subjects were included ( $n=65000$ glycogen particles). Note the normal distribution as well as the continuous range of particle diameter ( 9 to $43 \mathrm{~nm})$ in every subsarcolemmal location. Additionally, note the relative "left shift" of frequency distribution for the subsarcolemmal compared to the myofibrillar locations. 


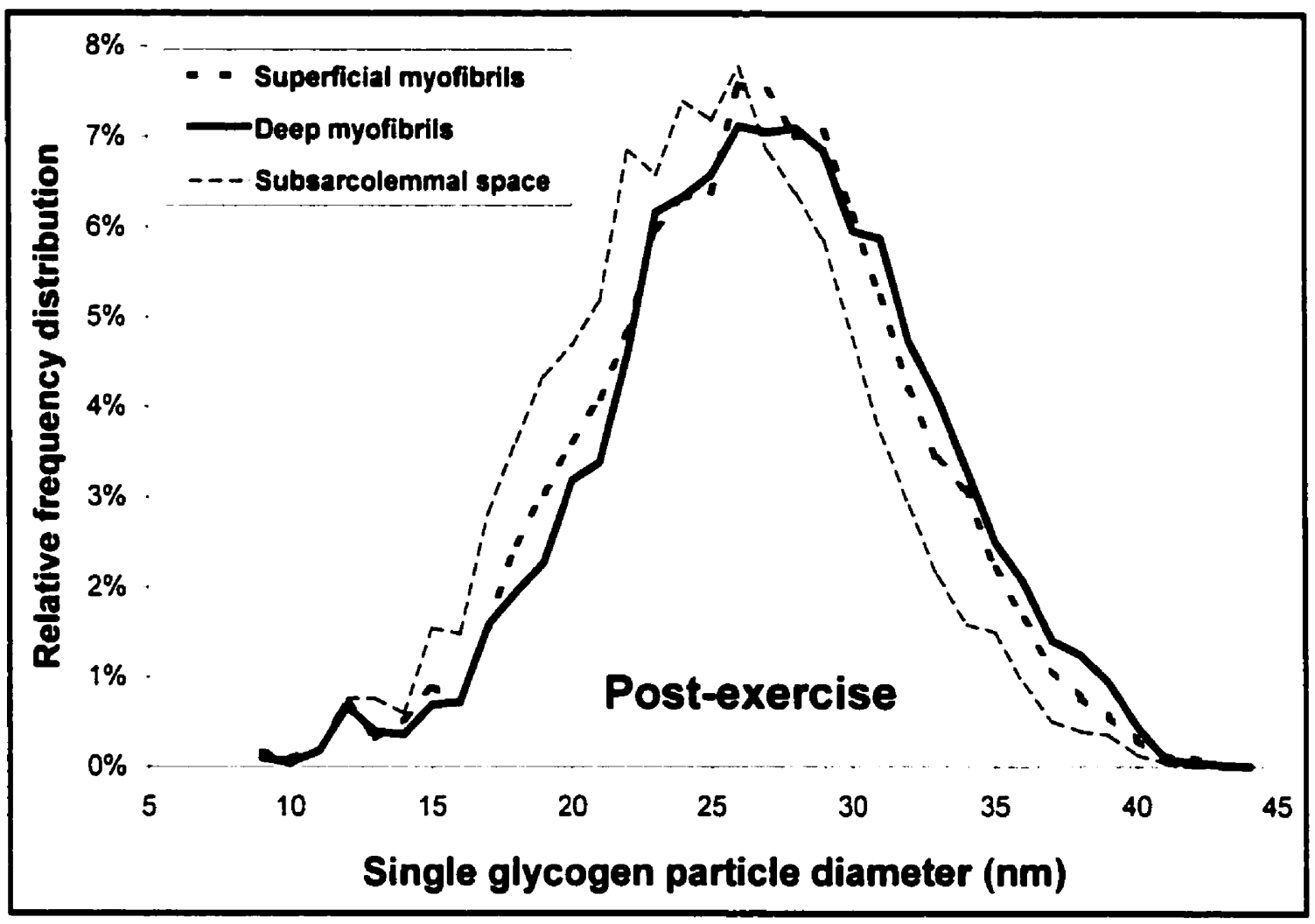

Figure 33 (B). Post-exercise relative frequency distribution of single particle diameter $(\mathrm{nm})$ in the three subcellular locations (subsarcolemmal space, superficial and deep myofibrils), across fibre type. The relative frequency distribution expresses the frequency as a percentage of the absolute frequency. The data from all six subjects were included ( $n=65000$ glycogen particles). Note the normal distribution as well as the continuous range of particle diameter ( -9 to $43 \mathrm{~nm}$ ) in every subsarcolemmal location. Additionally, note the relative "left shift" of frequency distribution for the subsarcolemmal compared to the myofibrillar locations.

Effect of fibre types.

Both single particle diameter and volume were found to differ according to fibre types (both $P=0.002$ ). Figure 34 illustrates the difference in single particle volume between fibre types. For clarity, the pre- and post-exercise data were pooled within each fibre type. The single particle volume was significantly greater in the type IIA (101 
$\left.\pm 7 \mu \mathrm{m}^{3} \times 10^{7}\right)$ and IIB $\left(103 \pm 9 \mu \mathrm{m}^{3} \times 10^{7}\right)$ than type I $\left(86 \pm 6 \mu \mathrm{m}^{3} \times 10^{7}\right)$ fibres. The corresponding values for single particle diameters were $25.5 \pm 0.6,26.7 \pm 0.6$ and $26.8 \pm$ $0.8 \mathrm{~nm}$, respectively. No difference was found between type IIA and IIB fibres for either variable. None of the other dependent variables were affected by fibre types.

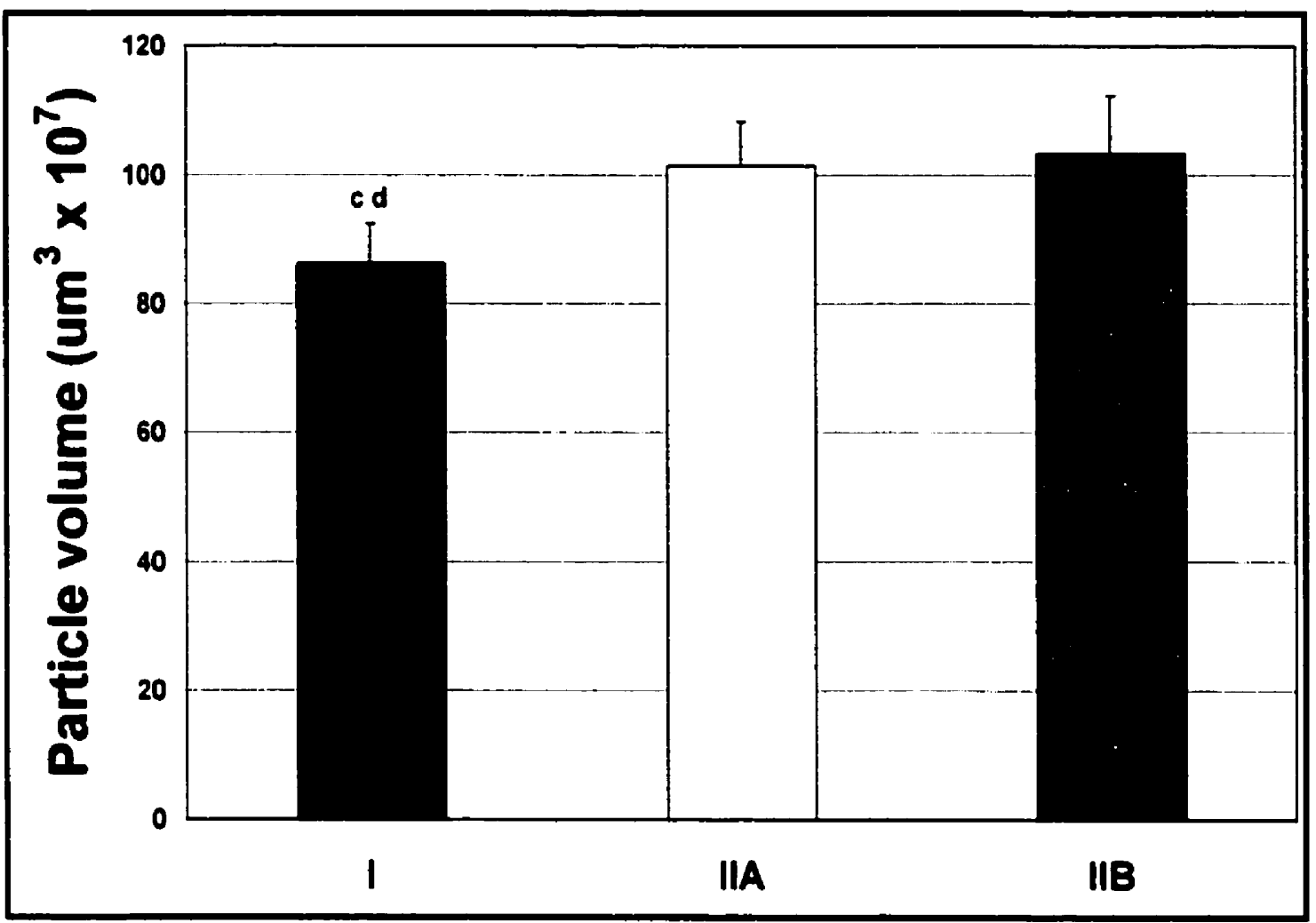

Figure 34. Effect of fibre type on single particle volume $\left(\mu \mathrm{m}^{3} \times 10^{7}\right)$. Values are means \pm S.E.M. The pre- and post-exercise data are pooled within each fibre type. A fibre type main effect was present $(P$ $=0.002$ ). : : significantly different from type IIA fibres $(p=0.01)$. d: significantly different from type IIB fibres $(p=0.004)$. There was no difference between type UA and $\| B$ fibres. 
Figure 35 illustrates the frequency distributions of pre-and post-exercise glycogen particle diameters. Figure 35 (A) represents the absolute frequency distribution of glycogen particles, as measured per $\mu \mathrm{m}^{3}$ of muscle tissue. While the exercise did not lead to a significant decrease in number of glycogen particles (Table 6), this figure illustrates the trend for a downward shift in the frequency of glycogen particles, particularly for those in the larger range $(>\sim 25 \mathrm{~nm})$. This suggests that, while particles from all size ranges were metabolised during exercise, the larger particles might be preferentially degraded.

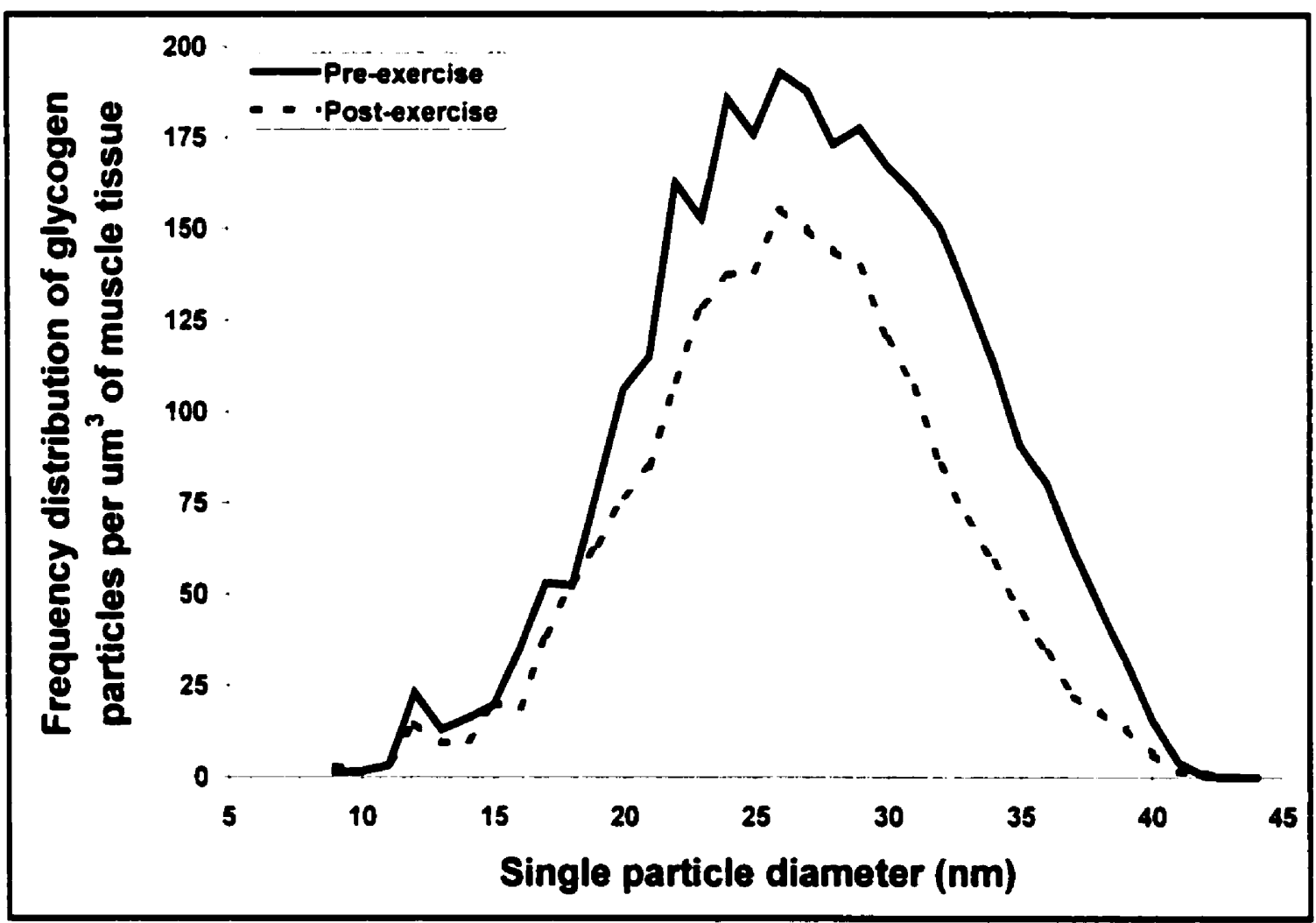

Figure 35 (A). Pre- and post-exercise absolute frequency distribution of glycogen particles, as measured per $\mu \mathrm{m}^{3}$ of muscle tissue. The pre- and post-exercise data, across subcellular locations and fibre types, from all six subjects were included ( $n=65000$ glycogen particles). 
Figure 35 (B) represents the relative frequency distribution of pre- and postexercise glycogen particle diameters. The relative frequency distribution expresses the frequency as a percentage of the absolute frequency. This allows comparing the preand post-exercise data while eliminating the effect of exercise on the number of particles. This figure illustrates that, on a relative basis, the frequency distribution of particles with a diameter greater than $\sim 32 \mathrm{~nm}$ displays a left-hand shift, indicating a degradation of these particles. On the other hand, the figure demonstrates the relative upward shift in the frequency of particles of intermediate sizes $(-22-32 \mathrm{~nm})$. This implies that a relatively greater percentage of the glycogen particles had a diameter between 22 and $32 \mathrm{~nm}$ following the exercise. 


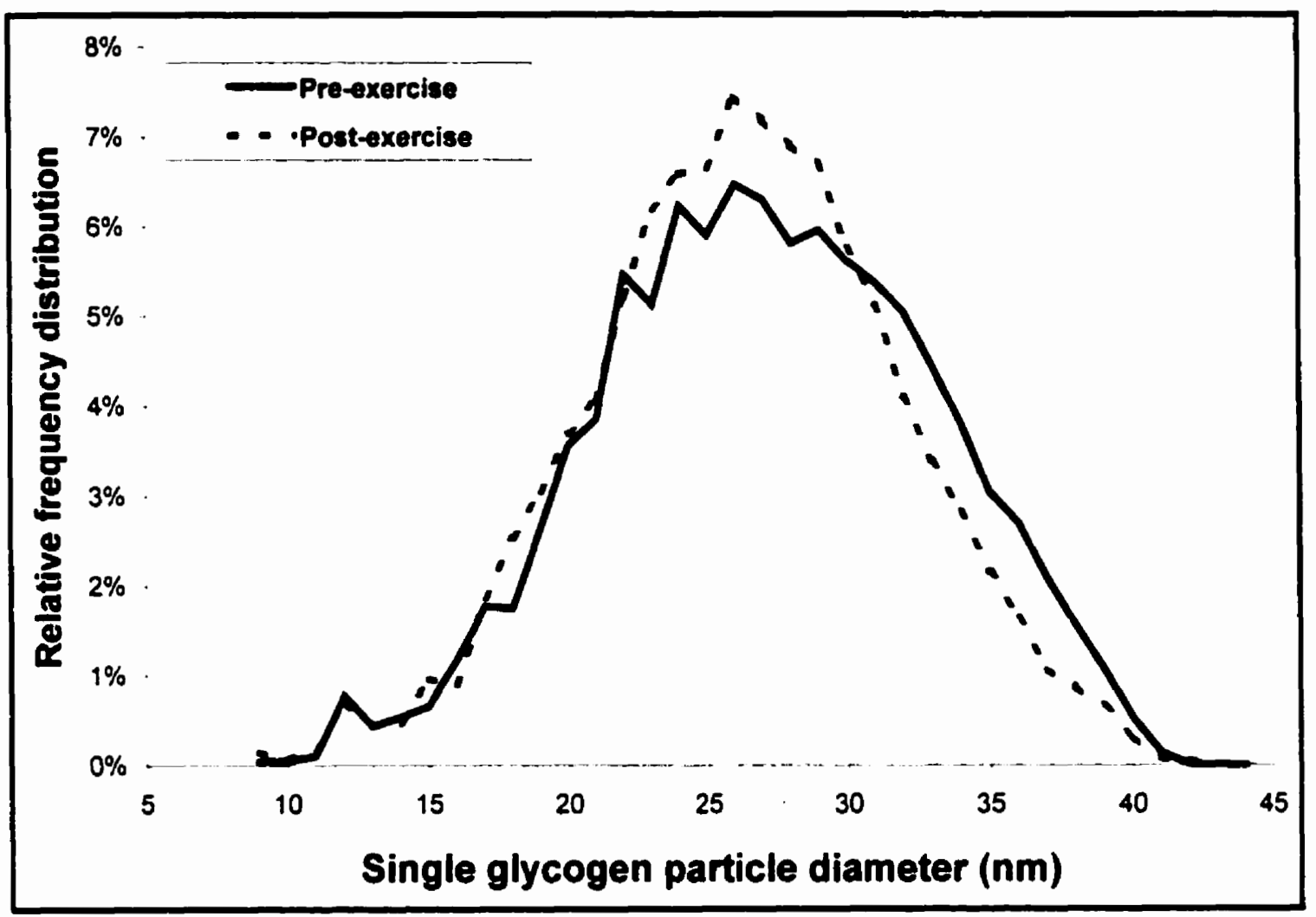

Figure 35 (B). Pre- and post-exercise relative frequency distribution of single particle diameter $(\mathrm{nm})$. The relative frequency distribution expresses the frequency as a percentage of the absolute frequency. The pre- and post-exercise data, across subcellular locations and fibre types, from all six subjects were included $(n=65000$ glycogen particles).

\section{Discussion}

The present experiment reports the first quantitative analysis of the subcellular distribution of human skeletal muscle glycogen during intense exercise. We hypothesised that myofibrillar and subsarcolemmal glycogen would be significantly degraded during the exercise, due to a decrease in both size and number of glycogen particles. The major findings include: 1) the exercise led to a significant decrease in both deep and superficial myofibrillar glycogen, while the decrease in the 
subsarcolemmal glycogen was not significant; 2) both pre- and post exercise, a continuum in single particle diameter distribution was observed in all subcellular locations; and 3) the exercise led to a trend for the number and size of the larger glycogen particles (> $32 \mathrm{~nm}$ ) to decrease preferentially compared to the smaller ones (< $20 \mathrm{~nm})$.

\section{Effect of exercise.}

The intense exercise performed in the present experiment caused a significant decrease in glycogen concentration, as demonstrated by both biochemical and TEMbased techniques (Table 6). The $46 \%$ decrease in total biochemically determined glycogen concentration (Figure 32) is similar to what has previously been reported during high intensity exercise (Bogdanis et al. 1996; Esbjornsson-Liljedahl et al. 1999; Graham et al. 2001; McCartney et al. 1986). The nature of our TEM-based technique allows quantifying the extent of glycogen degradation in the different subcellular compartments (Marchand et al. 2001). Hence, we were able to demonstrate that the exercise caused a 25, 30 and $53 \%$ decrease in subsarcolemmal, inter- and intramyofibrillar pre-exercise glycogen concentrations, respectively (Figure 32). This relative decrease in glycogen concentration (volume) was significantly different between subcellular compartments $(P=0.024)$, demonstrating a compartmentalised pattern of glycogen degradation during exercise. The decrease in subsarcolemmal glycogen volume was not significant $(P=0.16)$. Considering that the myofibrillar region corresponds to a much greater proportion of the total muscle tissue than does the subsarcolemmal space, during intense exercise the former is preferentially catabolized and contributes the majority of the carbohydrate.

While this represents the first quantitative analysis of the compartment-specific glycogen degradation during intense exercise, this phenomenon was previously qualified 
by the group of Ekblom and co-workers (Friden et al. 1985 and 1989; Sjostrom et al. 1982). They suggested that intense exercise caused a biased glycogen depletion in the subsarcolemmal and intra-myofibrillar compartments, whereas the inter-myofibrillar glycogen was relatively less affected (Friden et al. 1989; Sjostrom et al. 1982). The preferential depletion of the myofibrillar glycogen observed in the present study disagrees with this earlier observation, perhaps due to the qualitative nature of their analysis. On the other hand, the dissimilarity could by due to differences in exercise intensities. The protocol of the earlier studies (Friden et al. 1989 and Sjostrom et al. 1982 ) had a greater exercise intensity and total duration $(20 \times 25$-second or $60 \times 8$ second sprints ). Friden and colleagues (1985 and 1989) proposed that the role of the subsarcolemmal glycogen particles could be to secure energy yield for the maintenance of the plasma membrane potential and / or to act as an intra-cellular reserve energy supply for muscle contractions. If such is the case, it is possible that our exercise protocol was not intense enough to necessitate the use of this "intra-cellular reserve energy supply".

Our data agree more closely with their results for prolonged moderate-intensity exercise. They showed that this type of exercise leads to an extensive depletion of the glycogen located in the core of the depleted fibres (myofibrillar), while the subsarcolemmal glycogen is found to be relatively less utilised (Friden et al. 1985). These findings are similar to those of the present study and those reported previously (Chapter VI). Hence, it could be that a threshold in exercise intensity is required in order for the subsarcolemmal pool of glycogen to be catabolized at levels beyond that required for maintaining membrane potential. Further research is required to clarify the role of this glycogen pool in cellular function.

An alternate explanation for the fact that subsarcolemmal glycogen was not significantly decreased in the present study could be linked to our recent demonstration 
that the rate of glycogen synthesis is greater in the subsarcolemmal than the myofibrillar locations early during exercise recovery (Chapter VI). While previous studies have demonstrated the rate of total muscle glycogen resynthesis in the first 10 minutes after a bout of high-intensity exercise to be minimal (Bangsbo et al. 1991), it is possible that the compartment-specific subsarcolemmal synthesis rate was enhanced. Since the subsarcolemmal space represents a small fraction of the total muscle tissue, a specific glycogen synthesis in that compartment would not be detectable at the total muscle level. It is possible that the 10-minute rest period between the two 90-second sprint exercises allowed for subsarcolemmal glycogen to be synthesised to a more significant extent then the myofibrillar glycogen, hence minimising the effect of exercise contractions in decreasing subsarcolemmal glycogen volume.

While both myofibrillar compartments were degraded significantly during the exercise, a greater relative proportion of intra-myofibrillar glycogen was used (Figure 32 and Table 6) in agreement with earlier reports (Friden et al. 1989; Sjostrom et al. 1982). Pre-exercise, $13 \%$ of the myofibrillar glycogen was located in the intra-myofibrillar region. This value decreased to $10 \%$ immediately post-exercise $(P=0.0028)$. We recently showed that prolonged moderate intensity exercise similarly causes a preferential depletion of intra-myofibrillar glycogen, with the type 1 fibres' value decreasing to only $1 \%$ of the total myofibrillar glycogen (Chapter VI). Considering the close proximity of the glycogen pool to the contractile apparatus, these findings are to be expected. However, it should be acknowiedged that intra-myofibrillar glycogen represents a small proportion of the total muscle glycogen concentration (< $15 \%$ ).

The frequency distributions of glycogen particle diameter presented in Figure 35 (A) demonstrate a downward shift in the distribution from pre-to post-exercise. 
Furthermore, the extent of downward shift appeared to differ according to the range in particle diameters, being minimal in smaller particles and maximal in larger ones. This suggests that the biggest glycogen particles are preferentially degraded during intense exercise in human skeletal muscle. However, the $30 \%$ decrease in particle number (Table 6) was not significant $(P=0.11)$. While one could argue that a larger sample size would likely show significance if these tendencies are correct, the present data represent 65000 glycogen particles. Accordingly, it is most likely that an exercise protocol with an increased duration or intensity would create a greater metabolic demand and lead to a statistically detectable decrease in particle number.

To eliminate the influence of particle number on the frequency distribution of glycogen particle size, we expressed the frequency distribution in relative terms (Figure $35(B))$. The relative frequency distribution expresses the frequency as a percentage of the absolute frequency. If the degradation of glycogen particles during intense exercise was similar regardless of the particle diameter, then a leftward shift in the entire relative frequency distribution would be seen. This was not observed. Instead, only the upper end of the distribution was found to shift towards the middle. The relative frequency of particles with a diameter smaller than approximately $22 \mathrm{~nm}$ remained unaffected by exercise, implying that the proportion of pre- and post-exercise particles with a diameter smaller than $22 \mathrm{~nm}$ was similar. The particles ranging in diameter between 22 and 32 $\mathrm{nm}$ demonstrated a relative increase in frequency from pre- to post-exercise, while the largest particles (> $32 \mathrm{~nm}$ ) demonstrated a relative decrease in frequency. This means that a greater proportion of the post-exercise glycogen particles had a diameter between 22 and $32 \mathrm{~nm}$, while a lesser proportion had a diameter larger than $32 \mathrm{~nm}$. This implies that. while particles of all ranges showed a downward shift in the absolute frequency distribution (Figure $35(\mathrm{~A})$ ), the exercise led to a relative net accumulation of the 
glycogen particles of intermediate sizes and a relative net loss of the largest particles. Given that the carbohydrate content of glycogen particles increases exponentially with size (Goldsmith et al. 1982), the selective use of the larger particles is teleologically sound, representing a large liberation of glucosyl units.

It should be emphasised that a decrease in the frequency of particles of a given diameter could in theory be explained by both, the synthesis of these particles into larger ones or their degradation into smaller ones. Alternatively, the synthesis of smaller particles or the degradation of larger ones could cause an increase in the frequency of particles of a given diameter. The decrease in both the absolute and relative frequency distribution of particles larger than $32 \mathrm{~nm}$ is most likely caused by an increase in net degradation of these larger particles.

It is interesting to relate the size dependent behaviour of individual glycogen particles to the concept of pro- and macro-glycogen. Whelan and colleagues have proposed that PG is a 400000 Da stable intermediate in glycogen metabolism (Lomako et al. 1991). However, numerous investigations have provided evidence to disprove this hypothesis and to show that glycogen particles exist in a normally distributed continuum of sizes, with no accumulation of any major intermediate forms (Marchand et al. 2001; Skurat et al. 1997; Wanson and Drochmans 1968). Skurat et al. (1997) proposed that the findings by Lomako et al. (1991) were technical artefacts. We simply view PG and MG as smaller acid-insoluble and larger acid-soluble glycogen particles, respectively. Their behaviour in acid is determined by the ratio of protein and carbohydrate contents.

From the molecular mass and the glycogen structure proposed by mathematical modelling, it has been estimated that PG is a glycogen species containing a maximum of 8 tiers, compared to the fully grown MG which is believed to contain 12 tiers (Meléndez 
et al. 1997). Based on the estimated effective length per tier of $3.8 \mathrm{~nm}$, such a PG particle would have a diameter limited to below $30 \mathrm{~nm}$ (Goldsmith et al. 1982). However, the Whelan model for glycogen structure predicts that there is an exponential increase in the number of glucosyl units from tier to tier (50\% increase per tier) (Goldsmith et al. 1982). Therefore, a $30 \mathrm{~nm}$ PG would contain a maximum of approximately 10000 glucosyl units, that is $-18 \%$ of a fully-grown MG particle ( 55000 glucosyl units). Furthermore, when both glycogen species are compared in terms of molar concentrations, the MG particles represent less than $5 \%$ of total muscle glycogen particles (Derave et al. 2000), despite representing approximately $25 \%$ of the total muscle glucosyl units. Therefore, since resting skeletal muscles contain approximately $75 \%$ of their glucosyl units in the form of $P G$, and considering the frequency distribution of individual glycogen particles (Figures 33 and 35), it is clear that the $30 \mathrm{\eta m}$ value represents an underestimation of the actual maximal diameter of PG particles. Using our frequency distribution of particle diameters, the estimated effective length per tier of $3.8 \mathrm{~nm}$ (Goldsmith et al. 1982), the estimated number of glucosyl units per tier (Goldsmith et al. 1982), and the measured percentage of total glycogen in the PG and MG forms, we estimated the PG to MG transition to occur somewhere within the $10^{\text {th }}$ tier of glucosyl units. This corresponds to a maximal PG diameter between 32 and $36 \mathrm{~nm}$. Therefore, referring to Figure 35 , the separation between PG and MG occurs at a particle diameter between 32 and $36 \mathrm{~nm}$, the point of the frequency distribution below which $\sim 75 \%$ of the glucosyl units and $\sim 95 \%$ of the glycogen particles are located.

This is important with regard to the relative contribution of PG and MG in total muscle glycogenolysis during exercise. Hence, while $58 \%$ of the MG was degraded during the exercise (Figure 32), it only contributed to $31 \%$ of the glucosyl units provided for fuelling muscle contractions. The greater percentage of decrease in pre-exercise MG (58\%) compared to PG (44\%) in the present study is in agreement with most of the 
findings reported in the available literature in humans (Asp et al. 1999; Gaitanos et al. 1993; Graham et al. 2000; Shearer et al. 2001). However, while a greater percentage of MG is degraded during exercise, it should be emphasised that most of the glucosyl units provided for fuelling muscle contraction during exercise are derived from PG (69\%) and the present data demonstrate that this is most likely from the larger PG particles. We believe that this is explained by the greater availability of PG. As described above, MG represents less than $5 \%$ of the molar glycogen concentration. Hence, the PG particles are much more numerous than are the MG particles. While this represents an extremely efficient way of storing glucosyl units, the low number of particles may limit the ability of MG to contribute to the overall muscle glycogenolysis.

Taken together, and considering that $P G$ and $M G$ represent the smaller and higher range of glycogen particle sizes, the information provided in the present study suggest that the larger glycogen particles are preferentially catabolized over smaller ones during high intensity exercise. These larger particles appear to be all those particles larger than approximately $32 \mathrm{~nm}$, and represent all MG as well as the largest PG particles. The size of the particles preferentially catabolized during exercise is likely to differ according to the metabolic demand imposed upon the exercising muscles.

While the role of glycogen concentration in controlling glycogen synthase (GS) activity is well established (Nielson et al. 2001), controversy exists regarding its importance in controlling glycogen degradation during exercise (Vandenberghe et al. 1999). Recent evidence demonstrates that high muscle glycogen levels promote glycogen phosphorylase (GP) activity (Hespel and Richter 1992; Shearer et al. 2001; Vanderberghe et al. 1999). Furthermore, we recently provided evidence to suggest that the influence of glycogen levels on the control of net glycogen synthesis is accomplished at the level of individual glycogen particles (Chapter VI). Hence, depending on the size 
of individual glycogen particles, the interaction between the carbohydrate moiety and its associated enzymes would be altered, thus influencing the ability of specific enzymes to catalyse synthesis or degradation of individual particles. Therefore, in largest glycogen particles (>32 nm), the activity of GP could be enhanced compared to that of GS, thus favouring a net glycogen degradation. Conversely, glycogen particles with a smaller diameter could enhance the activity of GS over GP, hence favouring a net glycogen synthesis. Whether these differences in enzymatic activities are caused by alterations in enzyme activities or enzyme abundance, or both, is presently unknown. Taken together, these findings suggest that during high intensity exercise, the largest glycogen particles are degraded preferentially over smaller glycogen particles, and that this could be controlled at the individual particle level. This control of glycogen metabolism at the level of the individual glycogen particles, in addition to the compartmentalised pattern of glycogen degradation, could help explain the existing controversy regarding the role of glycogen concentration in influencing glycogen degradation during intense exercise.

The results presented in Table 6 demonstrate that the exercise-induced $8 \%$ decrease in particle volume was not significant. As mentioned above, the accepted model for glycogen structure predicts that there is a $50 \%$ increase in the number of glucosyl units from tier to tier (Goldsmith et al. 1982). This brings about a limitation of the present TEM-based technique in its ability to discriminate between the amount of glucosyl units liberated during the degradation of small and large diameters. Indeed, the degradation of the last tier of a particle with a diameter of $\sim 27 \mathrm{~nm}(\sim 8$ tiers) represents the liberation of approximately 3500 glucosyl units. This is in contrast to the liberation of 12500 glucosyl units caused by the degradation of the last tier of a particle with a diameter of $\sim 38 \mathrm{~nm}(\sim 11$ tiers). Hence, since most of the degradation occurred in particles with a larger diameter $(>32 \mathrm{~nm})$, it is possible that the non-significant statistical 
difference between pre- and post-exercise particle size, in fact, has a very significant physiological meaning.

Effect of subcellular location.

The novel approach used to study glycogen metabolism in the present experiment provides the unique ability to make distinctions between the different subcellular locations. Compared to both superficial and deep myofibrils, the subsarcolemmal space has a greater glycogen concentration generated by a greater number of smaller glycogen particles (Table 7). In fact, the number of glycogen particles was more than twice as great in the subsarcolemmal compared to the myofibrillar locations. These findings are in agreement with the findings of previous experiments using the same technique (Marchand et al. 2001 and Chapter VI). As pointed out previously, we must emphasise that while the glycogen in the subsarcolemmal space is more concentrated, this location represents a much smaller proportion of the total muscle tissue compared to the myofibrillar space. Therefore, the absolute amount of glycogen located in this compartment is much smaller relative to that contained in the myofibrillar tissue.

In agreement with our previous experiments, none of the variables examined was significantly different between the deep and superficial myofibrillar locations. However, as discussed earlier, it is possible that a physiologically significant difference in particle size between the superficial and deep myofibrils was not detected by our TEM-based technique. This would be related to the impact of particle size on the glucosyl unit density per tier, which is not taken into consideration in our analysis.

To further examine the difference of particle size between subcellular locations, we determined the relative frequency distribution of individual glycogen particle diameters (Figure 33). The leftward shifts observed in both the pre- and the post- 
exercise distribution of the subsarcolemmal compared to the myofibrillar glycogen particle diameters is clearly illustrated, and substantiate the subcellular location difference in particle size reported in Table 7. However, both pre- and post-exercise, the shape of the frequency distribution of particle diameter appears similar in subsarcolemmal and myofibrillar locations. This could be taken to suggest that, compareci to the myofibrillar locations, the balance between net particle synthesis and degradation is simply shifted towards degradation in the subsarcolemmal space. In turn, this could represent a greater glycogen turnover in that location. Interestingly, the subsarcolemmal space has been proposed to play a role in maintaining membrane potential (Friden et al. 1985 and 1989). Furthermore, under basal resting conditions, most of the GLUT-4 are found laced in the walls of intracellular vesicles (Klip et al. 1996). Hence, the only way for glucose to leave the blood stream and enter the skeletal muscle cells is through GLUT-1, which are exclusively located at the sarcolemmal membrane (Douen et 31.1990$)$. Taken together, our findings of smaller more numerous glycogen particles in the subsarcolemmal space, despite a similarly shaped particle frequency distribution and greater glycogen concentration, support the more dynamic role of subsarcolemmal glycogen in maintaining basal skeletal muscle cell functions.

\section{Effect of fibre types.}

It is well recognised that the extent of glycogenolysis in specific fibre types is dependent on exercise intensity (Essen 1978; Vollestad and Blom 1985). Accordingly, during sub-maximal light and moderate exercise, glycogen depletion is more pronounced in type I than type II fibres (Gollnick et al. 1974a and 1974b). On the other hand, at higher workloads, the type II fibres are glycogen depleted before type I fibre (Connett and Sahlin 1996). In agreement with this, the rates of glycogen depletion during maximal treadmill running have been demonstrated to be as much as two-fold 
greater in type II than type I fibres (Greenhaff et al. 1994). However, the results of the present study did not demonstrate a fibre type specific difference in glycogen degradation, as all three fibre types were equally affected by the intense sprint exercise (data not shown). In a previous experiment, we found a prolonged moderate-intensity exercise to lead to the degradation of type I and IIA fibres, while the type IIB fibres were relatively spared (Chapter VI).

In agreement with our previous experiments, we did not detect a difference in glycogen concentrations between fibre types. Although some studies reported no difference in resting glycogen levels between fibre types in human (Essen and Henriksson 1974), the majority of studies find a 16 to $40 \%$ greater glycogen content in type II compared to type I fibres (Casey et al. 1995; Eisenberg 1983; EsbjornssonLiljedahl et al. 1999; Essen 1978; Greenhaff et al. 1991, 1993 and 1994; Solderlund et al. 1992; Tsintzas et al. 1996; Vollestad et al. 1984 and 1989). The lack of fibre type difference in glycogen concentration in the present study could very well be linked to the fact that the glycogen particles differed between fibre types. Indeed, we found the particle size to be significantly smaller in type l compared to type II fibres (Figure 34). Therefore, given our inability to take into account the effect of particle diameter on glucosyl unit density per tier discussed earlier, and considering the significant fibre type difference in particle volume, we most likely minimised the fibre type difference in glycogen volume. That is, we did not take into account the lower density of glucosyl units in the type I fibre glycogen particles, which would have contributed to decreasing the glycogen concentration in that fibre type.

The smaller glycogen particle size found in type I fibres of the present study is in agreement with the results of our previous experiment performed in resting subjects (Marchand et al. 2001). It is interesting to speculate that, similarly to the phenomenon observed in the subsarcolemmal glycogen pool, the type I fibre glycogen could have a 
greater turnover in order to sub-serve more basic cellular functions or muscular contractions necessary to accomplish activities of daily living. In agreement with this, we previously showed that the different fibre types all possess a normal distribution of glycogen particle diameter, but that the type I distribution is shifted to the left (smaller sizes) (Marchand et al. 2001).

\section{Conclusion}

This study used a recently developed method for quantifying the subcellular distribution of skeletal muscle glycogen to characterise the pattern of glycogen depletion during high-intensity exercise. We confirmed our hypothesis that the glycogen in the different subcellular compartments is catabolized differently during high-intensity exercise. The results demonstrate that: 1) the exercise led to a significant decrease in both deep and superficial myofibrillar glycogen, while the decrease in the subsarcolemmal glycogen was not significant, 2) both pre- and post exercise, a continuum in single particle diameters distribution was observed in all subcellular locations, and 3) the exercise to led a tendency for the number and size of the larger glycogen particles (> $32 \mathrm{~nm}$ ) to decrease preferentially compared to the smaller ones (< $20 \mathrm{~nm}$ ). The dissociation observed in the metabolic pattern of glycogen particles of different sizes provide further evidence to our earlier suggestion that glycogen metabolism is regulated at the individual particle level. Finally, the current findings add to the emerging concept of the importance of subcellular compartmentalisation in the control of glycogen metabolism. 


\section{Chapter VIII}

General Thesis Discussion 
The focus of this thesis was to provide objective and quantitative ultrastructural data in the general area of human skeletal muscle glycogen and its metabolism. Standard biochemical experiments are responsible for most of the available knowledge in this field of research; however, information provided by direct visualisation using transmission electron microscopy (TEM) is unique and essential to complement the biochemical data. A few preliminary experiments have addressed the subcellular distribution of glycogen using TEM based techniques and, while their results are promising, the methodological approach used lacked the objectivity of quantitative procedures. Therefore, we developed a valid and reliable TEM-based method to quantify the subcellular distribution of human skeletal muscle glycogen, and applied it to a variety of experimental conditions. The major outcomes can be summarised as follows:

- We confirmed ealier suggestion that the distribution of human skeletal muscle glycogen is heterogeneous with respect to subcellular locations. The concentration of glycogen is greater in the subsarcolemmal compared to the myofibrillar compartment. The glycogen particles are smaller and more numerous in the subsarcolemmal compared to the myofibrillar compartment.

- The frequency distribution of individual glycogen particle sizes demonstrates that the particles present as a normal distribution ranging in diameter from 8 to $44 \mathrm{~nm}$. This normal distribution of particle sizes is observed in all subcellular locations and fibre types, and throughout a range from low to high glycogen concentrations. This provides direct evidence against the existence of PG as a stable intermediate in glycogen metabolism. 
- The distribution of human skeletal muscle glycogen is heterogeneous with respect to fibre types. The glycogen particles are smaller in the type I compared to type II fibres. The proportion of myofibrillar glycogen located in the intra-myofibrillar compartment is greater in type I compared to type II fibres.

- Both prolonged-moderate and short-intense exercises lead to the preferential depletion of myofibrillar over subsarcolemmal glycogen. In both types of exercise, a greater proportion of intra-compared to inter-myofibrillar glycogen is depleted. Since the myofibrillar compartment occupies a much greater proportion of total muscle tissue, and that the inter-myofibrillar compartment represents $>85 \%$ of this compartment, the latter is the principal storage source of glycogen used during exercise.

- Short-intense exercise leads to the preferential net degradation of the larger glycogen particles ( $>32 \mathrm{~nm}$ ), while the net degradation of smaller ones ( $<20 \mathrm{~nm}$ ) appears minimal.

- The glycogen resynthesis during the first four hours post-exercise is principally caused by an increase in the number of glycogen particles, while the glycogen increase in the remainder of recovery ( 48 hours) is characterised by an increase in the volume of individual particles.

- Following a prolonged-moderate intensity exercise, the net rate of glycogen synthesis in the first four hours post-exercise is greater in the subsarcolemmal compared to the myofibrillar subcellular compartment. In contrast, throughout the entire recovery period ( 48 hours), the net rate of single particle synthesis is greater in the myofibrillar compared to the subsarcolemmal compartment. Considering the greater glycogen concentration in the subsarcolemmal compartment, this dissociation between the net rate of synthesis for total glycogen and that at the level 
of the individual particle represents evidence suggesting that the control of glycogen synthesis is performed at the level of the individual glycogen particle.

The quality of information provided by our TEM-based technique is novel and can not be obtained by the existing standard biochemical technology. Taken together, these findings contribute and add significantly to the current knowledge of glycogen and its metabolism. The pattern of glycogen distribution described in the current thesis is in line with the emerging concept of subcellular organisation of the enzymatic machinery and metabolic pathways into an integrated compartmentalised glycogen metabolism.

Method validity and reliability.

One major contribution of the present thesis is that we have established a valid and reliable TEM-based technique to quantify the subcellular distribution of human skeletal muscle glycogen. As described in Chapter IV, our TEM-based measure of glycogen concentration correlates well with standard biochemical values of total muscle glycogen concentration. Our technique, although extremely time consuming when compared to traditional biochemical methods, provides the unique ability to discriminate between subcellular locations, as well as to quantify the distribution of glycogen in human skeletal muscle, with respect to particle size, number and concentration (volume).

We opted to present the glycogen concentrations in volume units $\left(\mu \mathrm{m}^{3}\right.$ of glycogen $/ \mu m^{3}$ of muscle tissue) as opposed to area units $\left(\mu m^{2}\right.$ of glycogen $/ \mu m^{2}$ of muscle tissue), as measured directly by our image analysis software. The rationale was simply to obtain a more representative value of glycogen concentration. However, a number of assumptions were necessary in order to convert glycogen area into glycogen 
volume. These included: 1) the glycogen particles are spheres, 2) the section thickness is $70 \mathrm{~nm}$, and 3) the sections for TEM have a maximal thickness equal to the average particle diameter.

The first two assumptions are valid. Glycogen particles, as a protein-glycogen complex, have been described consistently as regular spheroid organelles (Rybicka 1981; Goldstein et al. 1985; Rybicka 1996). The section thickness (70 nm) was precise within $\pm 10 \mathrm{~nm}$. Williams (1977) proposed to treat this problem of thin section thickness variability by examining a relatively large group of sections $(>30)$. By doing so, he stated that one could assume that the mean thickness of such a group is likely to be always similar. He suggested that this is likely to be sufficient for many comparative experiments, accepting that the true mean thickness will of course be known only approximately $( \pm 10 \mathrm{~nm})$. Since the total number of thin sections analysed in every one of our experiments is well above the minimal number proposed by Williams, we assume the mean thickness of our thin sections to be similar for every sample (Williams 1977).

The section thickness being approximately $70 \mathrm{~nm}$, and the average particle diameter around $25 \mathrm{~nm}$, it is possible that, especially within the clusters, one to three glycogen particles could be located on top of each other and escape detection. The two-dimensional TEM images could not detect this juxtaposition. We initially tried to evaluate the optical density of the glycogen particles. We compared the density of clusters to that of a single glycogen particle, assuming that clusters would be denser due to the effect of overlapping particles. However, a single isolated glycogen particle already displayed the maximal density. As detailed in the Chapter IV, we obtained the number of glycogen particles (per $\mu \mathrm{m}^{3}$ of muscle tissue) by dividing the glycogen area by the mean single particle area (both values obtained by direct measurement from the digitised images), and then by the thickness of a thin section $(0.070 \mu \mathrm{m})$ (Williams 
1977). The glycogen volume or concentration ( $\mu \mathrm{m}^{3} / \mu \mathrm{m}^{3}$ of muscle tissue) was then obtained by multiplying the mean single particle volume $\left(\mu \mathrm{m}^{3}\right)$ by the number of glycogen particles (per $\mu \mathrm{m}^{3}$ of tissue). Therefore, we elected for the conservative protocol of restricting the third dimension (thickness) to the maximal particle diameter times $70 \mathrm{~nm}$, for each particular image. We believe that to represent the best way of dealing with the issue of clusters of particles, which are not uniform in shape and most likely, do not represent glycogen particles pilled up in the full thickness of the thin section. Although we realise that this assumption introduces a certain degree of error in our measure of glycogen concentration, we are assuming that the error introduced is consistently present in all our samples and that, therefore, it will not bias our outcome.

A further limitation of our method relates to the fact that our total fibre value for glycogen concentration included only the deep and superficial myofibrils, and not the subsarcolemmal space. This is due to the fact that we were unable to determine the diameter of each individual muscle fibre. For this reason, it was not possible to determine the exact proportion of subsarcolemmal and myofibrillar tissue and therefore, to calculate a total fibre value that included both myofibrillar and subsarcolemmal glycogen. However, based on the fact that mammalian skeletal muscle fibres range in diameter between 10 and $100 \mu \mathrm{m}$ (Landon 1992), and assuming that the muscle fibre is a sphere in cross section and that the subsarcolemmal space represents the muscle tissue located within $1 \mu \mathrm{m}$ of the sarcolemma (Ejsenberg and Kuda 1975), we can estimate the contribution of the myofibrillar space to the total muscle tissue. Following is a summary of these calculations: 
Calculations for the proportion of subsarcolemmal space to total myofibrillar tissue

$\%$ myofibrillar tissue $=\frac{\pi \times r_{1} \times r_{1} \times 100}{\pi \times r_{2} \times r_{2}}=\frac{r_{1} \times r_{1} \times 100}{r_{2} \times r_{2}}$

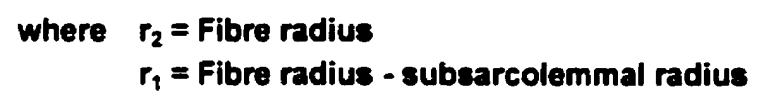

If fibre diameter $=10 \mathrm{um}$

$\%$ myofibrillar tissue $=\frac{4 \times 4 \times 100}{5 \times 5}=\frac{16 \times 100}{25}=64 \%$

If fibre diameter $=50 \mathrm{um}$

$\%$ myofibrillar tissue $=\frac{24 \times 24 \times 100}{25 \times 25}=\frac{576 \times 100}{625}=92 \%$

If fibre diameter $=100 \mathrm{um}$

$\%$ myofibrillar tissue $=\frac{49 \times 49 \times 100}{50 \times 50}=\frac{2401 \times 100}{2500}=96 \%$

Hence, we estimated that the proportion of total muscle tissue as myofibrillar tissue ranges between 64 and $96 \%$ of the total muscle tissue, for fibre diameters of 10 and $100 \mu \mathrm{m}$, respectively. Furthermore, considering the reported average fibre diameter for men between 20 and 30 year old of $50 \mathrm{~nm}$ (Lexell et al. 1988), the proportion of muscle tissue as myofibrillar tissue is $\sim 92 \%$. Therefore, based on those calculations, we consider that the myofibrillar value represents a valid estimate of the total muscle fibre value. 
Frequency distribution of particle diameters.

An important contribution of the present thesis is the evidence that the frequency distribution of individual particle diameter approximate very closely the standard normal distribution. As discussed in Chapter V, the distribution is slightly skewed to the left due to the fact that a small proportion of particles will ultimately be cut in such a way that it will lead to an underestimation of their actual diameter. This continuum of particle size is observed in all fibre types (Chapter V), all subcellular locations (Chapter VII), and throughout a range of low (13 mmol glucosyl units / $\mathrm{kg} \mathrm{dw}$ ) to high (692 mmol glucosyl units / kg dw) glycogen concentrations (Chapter VI). Furthermore, the particles range in size from approximately 9 to $44 \mathrm{~nm}$. These quantitative findings, based on the more than 250000 individual glycogen particles, substantiate and extend earlier qualitative observations that glycogen $\beta$ particles appear as roughly spherical in shape, and are 15 to $40 \mathrm{~nm}$ in diameter (Biava 1963; Revel 1964; Rybicka 1996). Furthermore, the present findings also confirm the normal distribution of glycogen $\beta$ particles suggested by earlier investigations (Scott and Still 1968; Wanson and Drochmans 1968).

Due to its complexity, the structure of glycogen particles has been modelled mathematically (Goldsmith et al. 1982). Based on the accepted model of glycogen structure (Goldsmith et al. 1982) and the molecular weight of rabbit muscle glycogen $\beta$ particle $\left(10^{7} \mathrm{Da}\right)$ reported by Wanson and Drochmans (1968), glycogen is believed to present as a regular spherical structure organised into a maximum of 12 concentric tiers, with a maximal diameter of $42 \mathrm{~nm}$ and a total of 55000 glucosyl units. It is striking how close our value of maximal particle diameter fits with these numbers. The information provided by Goldsmith and colleagues (1982) allowed us to predict the diameter, the number of glucosyl units, as well as the molecular weight of glycogen particles of different number of tiers. Table 8 represents a summary of these estimates. 
Table 8.

\begin{tabular}{cccc}
\hline \hline Tier \# & $\begin{array}{c}\text { Diameter \# Glucosyl units } \\
\text { (nm) }\end{array}$ & $\begin{array}{c}\text { Cumulative } \\
\text { per tier }\end{array}$ & \# Glucosyl unit \\
\hline \hline 3 & 7.8 & 417 & 1205 \\
4 & 11.6 & 637 & 1842 \\
5 & 15.4 & 974 & 2816 \\
6 & 19.2 & 1489 & 4305 \\
7 & 23.0 & 2277 & 6582 \\
8 & 26.8 & 3481 & 10063 \\
9 & 30.6 & 5323 & 15386 \\
10 & 34.4 & 8139 & 23525 \\
11 & 38.2 & 12445 & 35970 \\
12 & 42.0 & 19030 & 55000 \\
\hline \hline
\end{tabular}

As predicted by the mathematical modelling, the number of glucosyl units increases exponentially from tier to tier ( $\sim 50 \%$ increase per tier). This is related to the branching pattern of the glycogen particles, as discussed in Chapter II. Therefore, the number of glucosyl units in any given outermost tier is always $-35 \%$ of the total number of glucosyl units included in that glycogen particle, regardless of the particle size. This increase in density as the glycogen particle grows is believed to be responsible for the physical limit of the glycogen particle size (Goldsmith et al. 1982).

When compared to the $42 \mathrm{~nm}$ estimate obtained by mathematical modelling (Table 8), the maximal glycogen particle diameter obtained by our TEM-based technique is slightly larger ( $44 \mathrm{~nm})$. We explain this difference by the nature of our technique used to enhance skeletal muscle glycogen. Indeed, while the exact chemical natura of the reaction involved in not completely understood, potassium ferrocyanide is known to enhance the visualisation of glycogen over other cellular components by binding to the 
attached metabolising enzymes (Rybicka 1996). Hence, since the dimensions obtained from the mathematical models do not include the weight and size of the different enzymes that are known to be associated with the glycogen particle, we believe that the slightly larger diameter obtained by our technique reflects the association of these enzymes with the glycogen particle to form glycosomes (Rybicka 1996).

To illustrate the mathematically predicted exponential increase in number of glucosyl units per tier, we plotted the number of glucosyl units per tier in a given glycogen particle (Figure 36). Refer to Table 8 for the corresponding diameter per tier.

Figure 36. Number of glucosyl units per tier and a glycogen particle.

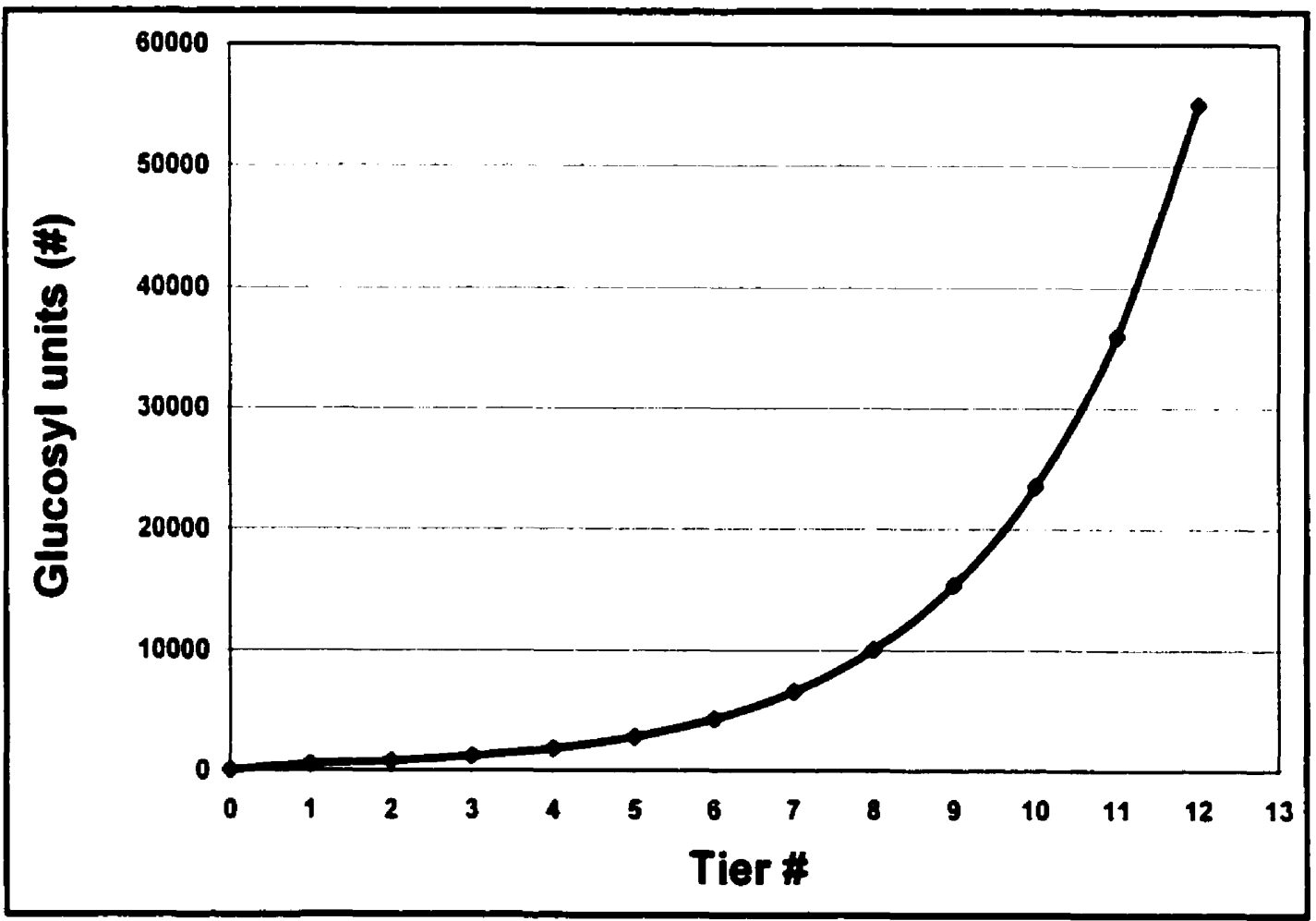


From this figure, it is clear that a change in particle size from tier 7 to 8 is physiologically much less significant than a change in particle size from tier 10 to 11 . This is important in interpreting the significance of the findings from every one of our experimental conditions (Chapters V to VII). To further accentuate the physiological importance of this issue in the integration of our data to the existing literature on glycogen metabolism, we plotted Figure 37. Figure 37 represents the data from 11 resting subjects (Chapter V). In this figure, based on the calculations presented in Table 8, we juxtaposed the relative frequency distribution of individual glycogen particles and the corresponding number of glucosyl units per tier. The number on top of each bar represents the corresponding diameter in nm. The arrow represents the pro- (PG) to macro-glycogen (MG) transition and will be addressed subsequently. The rightward shift in the frequency distribution of glucosyl units compared to that of the number of glycogen particles demonstrates well the concept of the greater storage contribution of glucose units in larger glycogen particles compared to smailer ones. Indeed, while more than $20 \%$ of the glycogen particles in a given muscle with resting glycogen concentrations have a diameter of $\sim 23 \mathrm{~nm}$ ( 7 tiers), the glucosyl units stored in those particles represent less than $12 \%$ of the total muscle glucosyl units store. Conversely, while only $4 \%$ of the glycogen particles in the same muscle sample have a diameter of $-38 \mathrm{~nm}$ ( 11 tiers), about $13 \%$ of the total muscle glucosyl units are stored in those particles. Furthermore, based on Table 8, the degradation of the last tier of a particle with a diameter of $\sim 27 \mathrm{~nm}(\sim 8$ tiers) represents the liberation of approximately 3500 glucosyl units, while the degradation of the last tier of a particle with a diameter of $\sim 38$ $\mathrm{nm}(\sim 11$ tiers) liberates approximately 12500 glucosyl units. The implications of this concept are described for each experimental condition in the appropriate Chapter ( $V$ to VII). 


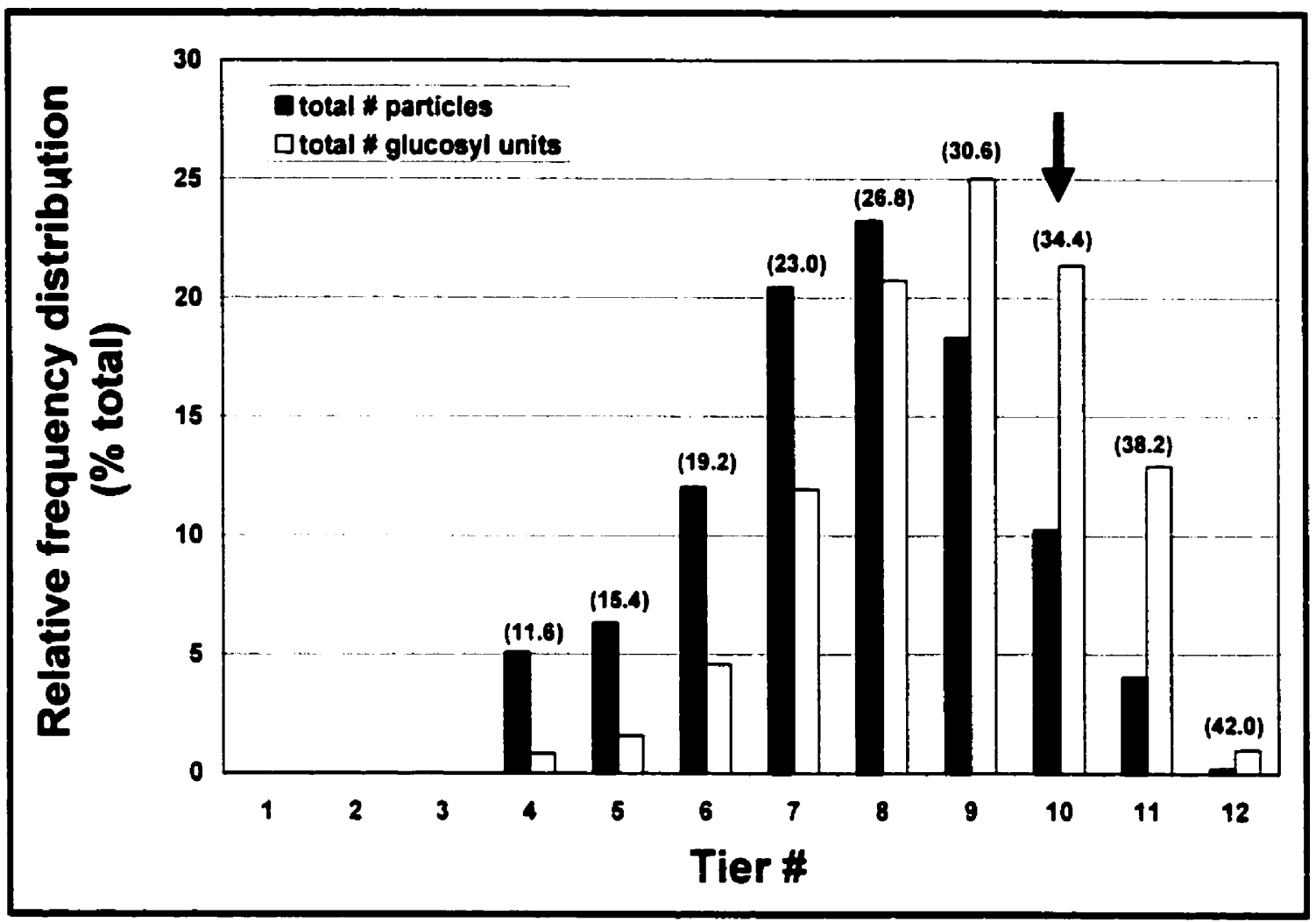

Figure 37. Relative frequency distribution of single particle combined with the corresponding number of glucosyl units per tier. The relative frequency distribution expresses the frequency as a percentage of the absolute frequency. The data from all 11 subjects were included (Chapter $V$ ). The number in brackets above each column represents the diameter in $\mathrm{nm}$. The arrow represents the estimated diameter at which the transition PG to MG occurs. This is further discussed in the text.

It is interesting to relate these observations to the concept of PG and MG. Whelan and colleagues have proposed that PG is a $400000 \mathrm{Da}$ stable intermediate in glycogen metabolism (Lomako et al. 1991). In their original experiment, Lomako and colleagues (1991) studied the migration of the $C^{14}$ glucose-labelled products by discontinuous electrophoresis in a $10 \%$ polyacrylamide gel. However, while Skurat et al. (1997) were able to reproduce these results using the same method, they also used SDS / PAGE in a continuous buffer system and revealed that this product of $\mathrm{C}^{14}$ 
glucose-labelled was not a discrete entity. Rather, Skurat and co-workers (1997) showed that glycogen particles exist as a continuum of glycogenin-containing species from low molecular mass to sizes significantly greater than $400 \mathrm{KDa}$, hence disproving the presence of the accumulation of any major intermediate form of glycogen particles. Our finding of a normal frequency distribution of glycogen particle sizes substantiates the results of Skurat and colleagues (1997) and provides direct evidence against the existence of PG as a discrete glycogen species. Based on these observations, we view $P G$ and MG as smaller acid-insoluble and larger acid-soluble glycogen particles, respectively. Their behaviour in acid is determined by the ratio of protein and carbohydrate contents.

Furthermore, based on the initial findings by Lomako and colleagues (1991), it has been assumed that the transition from PG to MG occurs somewhere when the glycogen particles are in their $8^{\text {th }}$ tier $(<30 \mathrm{~nm}$ ) (Meléndez et al. 1997). However, given the results presented in Table 8 and Figure 37, and considering that $\sim 75 \%$ of resting skeletal muscie glycogen is in the form of PG, it is clear that this estimation is erroneous. Accordingly, based on Table 8, we estimate the transition from PG to MG to occur somewhere within the $10^{\text {th }}$ tier (Arrow in Figure 37). Accordingly, while MG represents approximately $25 \%$ of the glucosyl units in resting skeletal muscles, it represents at most $10 \%$ of the molar concentration (or number of glycogen particles). As pointed out by Derave and colleagues (2000), this has important implications regarding the abundance of the enzyme glycogen phosphorylase (GP). As discussed in the previous section, a statistically non significant decrease in size in a MG particle represents the release of a much greater number of glursosyl units than a larger decrease in size in a PG particle. Unfortunately, the molarities of skeletal muscle glycogen and the stoichiometry of GP to glycogen particles of different molecular weights (i.e. PG and 
MG) are poorly understood (Derave et al. 2000). The impact of this during exercise is discussed in Chapter VII.

Figure 38 constitutes an interesting complement to the findings presented in Chapter V to VII. It illustrates the preliminary results of an analysis comparing the frequency distribution of glycogen particle diameter in a normal glycogen-loaded subjects and a patient affected with McArdle's disease. The McArdle's disease (muscle glycogen phosphorylase deficiency) is the most common glycogenosis affecting skeletal muscle (Martinuzzi et al. 1999). The biochemical hallmark of the disease is impaired muscle glycogenolysis due to the lack of muscle glycogen phosphorylase activity (Martinuzzi et al. 1999).

Figure 38.

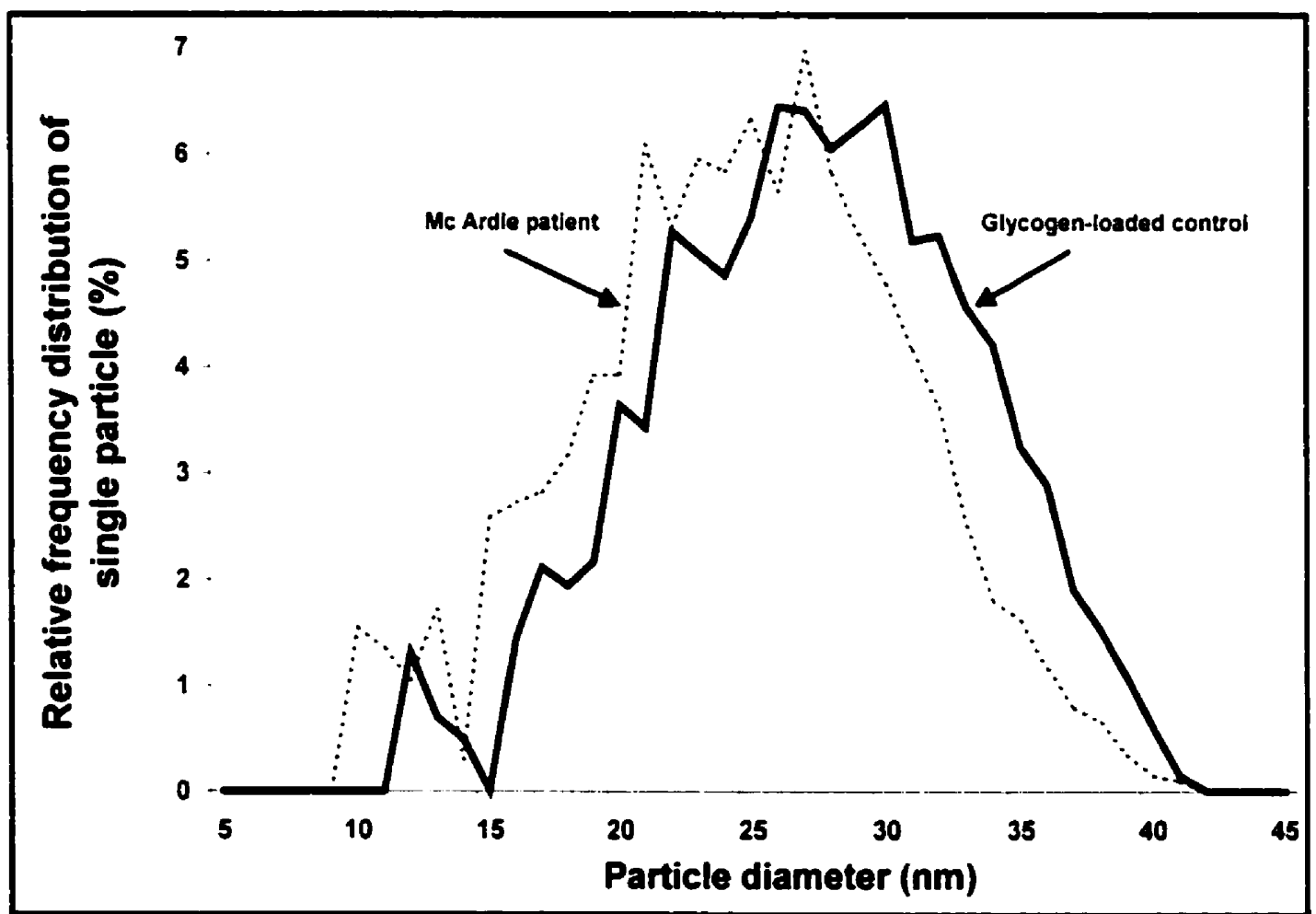


Both subjects had a similar biochemically determined glycogen concentration $(\sim 670 \mathrm{mmol}$ glucosyl units $/ \mathrm{kg} \mathrm{dw})$. The leftward shift in the frequency distribution of the McArdle's patient is striking. Accordingly, the mean particle diameters were 27 and 24 $\mathrm{nm}$ for the glycogen-loaded subject and patient, respectively. Referring to table 8 , this difference in mean single particle diameter represents a difference of more than 3000 glucosyl units per individual glycogen particles. This is surprising considering the similar total muscle glycogen concentration. It is interesting to speculate that the difference in size could be linked to the absence of GP association with the complex. GP and other enzymes involved in glycogen metabolism, are known to be associated with the glycogen particle to form glycosomes (Meyer et al. 1970; Nelson et al. 1972; Rybicka 1996; Taylor et al. 1975). In an original study by Madsen and Cori (1958), it was suggested that maximums of 20-25 GP are associated with each glycosome. Furthermore, they were able to show that the removal of GP from glycosomes yielded a glycogen particle of smaller $M_{r}$ and suggested that this could account for their smaller size (Madsen and Cori 1958). Therefore, it is possible that the glycosomes of McArdle patients, which are not associated with GP (or at least in a decreased amount), appear as a smaller size. This would shift the frequency distribution to the left (Figure 38), despite a similar number of glucosyl units.

The other important differences between the patient and glycogen-loaded control subject relate to the subcellular distribution of the glycogen in the different compartments (data not shown). Indeed, the ratio of subsarcolemmal to myofibrillar glycogen was almost $50 \%$ less in the patient $(0.70)$ than the glycogen-loaded subject (1.26). Furthermore, the proportion of myofibrillar glycogen located in the intramyofibrillar compartment was $16.3 \%$ in the patient and 13.3 in the glycogen-loaded subject. These results suggest that the subsarcolemmal compartment represents a less 
important store of skeletal muscle glycogen in those patients. Conversely, a greater proportion of total muscle glycogen is stored in the intra-myofibrillar compartment in those same patients. Whereas these results are based on only two samples, they certainly suggest important differences in the subcellular distribution of skeletal muscle glycogen in patients lacking GP activity. While the physiological significance of these preliminary findings is unclear, a more comprehensive analysis will help clarify these interesting observations.

\section{Subcellular glycogen distribution.}

Perhaps the most consistent of our findings relates to the heterogeneity of the glycogen distribution in the different subcellular compartments. In every experimental condition, the glycogen volume was significantly more concentrated in the subsarcolemmal compared to both the deep and superficial myofibrillar compartments. Furthermore, this greater glycogen concentration was accomplished by more numerous glycogen particles that were smaller in size. In addition, there was a tendency for the glycogen in the superficial myofibrils to be composed of smaller, more numerous, glycogen particles compared to the deep myofibrils, despite similar glycogen concentrations. Our findings represent the first evidence of a graded increase in single glycogen particle size (and decrease in particle number) from the sarcolemma to the centre of the muscle fibre. While the physiological significance of this is unknown, it stimulates a series of speculations.

Since glycogenin represents the protein backbone of every glycogen particle in skeletal muscles, an increased number of glycogenin molecules must be present to cause an increase in number of glycogen particles. Accordingly, recent evidence suggests that glycogenin over-expression leads to the production of more, smaller, glycogen particles (Hansen et al. 1998; Skurat et al. 1997). However, the effect of 
glycogenin over-expression on total glycogen concentration is controversial, with one study reporting no change (Skurat et al. 1997), and one reporting a small increase (Hansen et al. 1998). The nucleus being located almost exclusively in the subsarcolemmal space of skeletal muscles, it could encode the nuclear genome of glycogenin, translate it into the cytosol, and import it to the final subsarcolemmal destinations in the subsarcolemmal space potentially more easily compared to the deeper myofibrillar locations. The physical distance from the nucleus could limit glycogenin availability in the myofibrillar compared to the subsarcolemmal locations, and hence lead to less numerous, bigger, glycogen particles in the former locations. On the other hand. ample availability of glycogenin in the subsarcolemmal space would lead to a greater number of glycogen particles, albeit smaller in size, despite a greater glycogen concentration. This could explain the descending gradient in glycogen particle number from the sarcolemma to the deep myofibrils. Such specificity in nuclear-directed protein synthesis has been reported in the different mitochondrial fractions (Cogswell et al. 1993). Cogswell and colleagues (1993) suggested that specific gene products could be preferentially targeted to specific mitochondrial fractions, leading to higher enzyme activities in certain mitochondrial fractions.

Interestingly, this greater glycogenin availability for the subsarcolemmal compared to the myofibrillar locations could also explain the greater net rate of single particle synthesis in the subsarcolemmal compared to the myofibrillar spaces (Chapter VI). In agreement with this, the superficial myofibrillar glycogen was also found to have a smaller net rate of glycogen particle synthesis compared to the deep myofibrillar space. This finding supports the notion that the physical distance from the nucleus could in fact limit glycogenin activity, and hence lead to less numerous, bigger, glycogen particles. 
Referring to the results presented in Chapter VI, this greater glycogenin availability could also explain the higher rate of glycogen synthesis in the subsarcolemmal space, despite a lower rate of increase in particle size. Therefore, more particles being synthesised simultaneously in the subsarcolemmal space, due to a greater glycogenin availability, would lead to a slower increase in individual glycogen particle size, despite a higher overall rate of glycogen synthesis. Interestingly, a recent study by Shearer and colleagues (2000) demonstrated that glycogenin activity, as measured by glycogenin protein content, is proportional to muscle glycogen concentration. If the control of glycogen concentration is compartmentalised, it is likely that the hypothesised greater glycogenin concentration in the subsarcolemmal space compared to the myofibrillar ones would be responsible for the greater glycogen concentration in that space. However, we are unaware of any direct data regarding the subcellular distribution of glycogenin. Further studies are required to clarify the role of glycogenin in subcellular distribution of skeletal muscle glycogen.

The metabolic implications of these findings are presently unknown. However, a gradient of inter-myofibrillar mitochondrial content, from high to low, has previously been reported from the sarcolemma to the centre of the muscle fibre (Kayar et al. 1986). Interestingly, it has been shown that alterations in energy demand, such as those imposed by contractile activity, play a large role in determining the intracellular distribution and content of mitochondria in skeletal muscles (Cogswell et al. 1993). In fact, numerous examples exist whereby increases or decreases in muscle activity have resulted in differential adaptations of mitochondria located in the subsarcolemmal and inter-myofibrillar regions (Cogswell et al. 1993). The responses suggest that these mitochondria have different functions related to metabolic compartmentalisation (Cogswell et al. 1993). Such compartmentalisation of biochemical properties has been 
suggested to serve specific regional functions (i.e. plasma membrane vs. contractile elements) related to substrate availability or ATP requirements.

Accordingly, it has been previously suggested that the subsarcolemmal glycogen particles could be stored in that location to secure adequate energy store for the maintenance of the plasma membrane potential and / or to act as an intra-cellular energy reserve (Friden et al. 1985 and 1989). In resting conditions, the main pathway for glucose to enter the skeletal muscle cells is through GLUT-1, which are exclusively located at the sarcolemmal membrane. GLUT-1 have been suggested to regulate glucose transport into the muscle under basal conditions (Douen et al. 1990). Furthermore, the results presented in this thesis demonstrate that the myofibrillar glycogen is preferentially used over the subsarcolemmal one during both prolongedmoderate and short-intense exercise (Chapter VI and VII). Taken together, these observations tend to support the hypothesis of Friden and colleagues (1985 and 1989) that the subsarcolemmal glycogen serves mainly to support plasma membrane potential, although further research is necessary to establish this. While the present study does not allow us to unequivocally confirm this hypothesis, our finding of smaller more numerous glycogen particles in the subsarcolemmal space, despite a greater glycogen concentration, certainly points towards an increased glycogen turnover in that compartment compared to the myofibrillar glycogen.

Our findings of a graded increase in particle size (and decrease in particle number) from the sarcolemma to the centre of the fibre, in addition to the exercise specific use of myofibrillar glycogen, could be taken to suggest that glycogen particles, like mitochondria, are compartmentalised within skeletal muscles. The different glycogen fractions in the muscle could possess different biochemical properties, which may serve functions specific to their respective compartments. If one considers that the glycogen particles are in fact organelles of their own (Rybicka 1996), this 
compartmentalisation could perhaps be related to specific regional (i.e. plasma membrane vs. contractile elements) substrate availability or metabolic demand.

Another aspect of subcellular glycogen distribution relates to the I band bias of both intra- and inter-myofibrillar glycogen fractions. Friden and co-workers (1989) suggested that this inter-myofibrillar I band bias of skeletal muscle glycogen could be related to the fact that the I band represents the portion of the sarcomeres with the highest sarcoplasmic reticulum (SR) density. In fact, the glycogen-enzyme complex (glycosome) has consistently been demonstrated to be physically associated with the SR (Goldstein et al. 1985; Rybicka 1981a and 1981b; Wanson and Drochmans 1968 and 1972; Whelan 1970). In addition, GS and glycogenin have also been shown to bind the SR independent of the protein-glycogen complex (Polishchuk et al. 1995). Furthermore, the regulatory subunit $\left(R_{\mathrm{GL}}\right)$ of the muscle protein phosphatase 1 (PP1) has been found to bind to the SR through its strongly hydrophobic region (Tang 1991). Hubbard and Cohen (1997) suggested that the targeting of $R_{G L}$ to both glycogen and the SR might contribute to the long-recognised physical association of these structures. While the exact physiological meaning of these protein interactions remains to be clarified, the interaction between glycosomes and SR, in close proximity to the transverse tubular system where the GLUT-4 are known to translocate in response to insulin (Lund et al. 1993) and exercise (Cartee et al. 1989), could facilitate the synthesis of glycogen by increasing glucose availability. Furthermore, the ability of the SR to sequester $\mathrm{Ca}^{2+}$ provides a potential mechanism for allosteric regulation of some of the glycogen metabolising enzymes (Kruszynska et al. 2001). In fact, a role for calcium sequestration by the SR in controlling glycogen phosphorylase has been demonstrated (Entman et al. 1980). Interestingly, the complete chain of glycolytic enzymes has also been demonstrated to be anatomically associated with the SR membranes (Xu and Becker 
1998). This could represent an efficient means of directing the product of glycogen degradation (glucose and phosphate) directly into the ATP producing system that is glycolysis.

In summary, the present quantitative analysis of intra-muscular glycogen distribution has provided essentially novel information concerning a compartmentalised / heterogeneous pattern of glycogen distribution. These data are in line with the evidence of a compartmentalisation of different mitochondrial fractions, as well as with the emerging concept of the subcellular organisation of the enzymatic machinery and metabolic pathways into an integrated compartment-based glycogen metabolism.

Fibre types.

The results presented in this thesis demonstrate that the distribution of skeletal muscle glycogen is heterogeneous with respect to fibre types. While we were unable to detect fibre type difference in resting glycogen concentrations, the type I fibres were found to have smaller, more numerous glycogen particles. Furthermore, the type I fibres were found to express a greater proportion of their myofibrillar glycogen in the intramyofibrillar compartment compared to type II fibres.

The lack of difference in resting glycogen concentration between fibre types was consistent in every one of our experimental conditions. Based on the literature, the differences in glycogen concentration between type I and type II fibres are greater when obtained by standard biochemical measures (II > I by 17 to $40 \%$ ) (Casey et al. 1995; Essen 1978; Tsintzas et al. 1996) than when obtained with PAS staining (II > I by $16 \%$ ) (Vollestad et al. 1984). Although histochemical in nature, our TEM-based technique is quantitative and we would expect to detect such a difference. One possible explanation 
is that our fibre typing procedure is not valid and hence, hides the fibre type effect on glycogen concentration. We believe this to be unlikely. Although we did not validate our fibre typing procedure by standard myosin ATPase identification methods, others have, and showed our procedure to be a valid tool for identifying the fibre types (Prince et al. 1981; Sjostrom et al. 1982b; Payne et al. 1975). Sjostrom et al. (1982b) showed that 70 to $80 \%$ of the fibres are correctly allocated on the basis of the Z-line width alone. They reported that all the fibres that were not allocated to the proper type concerned the IIA fibres, being falsely allocated to either type I or type IIB. No error of allocation was found between type $\| \mathrm{B}$ and $\mathrm{I}$. We therefore improved this by considering other criteria (see Chapter IV - Detailed methods) which have also been shown to be valid (Eisenberg 1983; Prince et al. 1981). Therefore, the procedure that we used should allocate significantly more than $80 \%$ of the fibres to their proper histochemical types.

Another possibility is that our technique lacks adequate precision. As presented in Chapter IV, the coefficient of variation for glycogen determination is in the order of 10 $15 \%$. The expected fibre type difference in glycogen concentration, as reported above, is between 17 and $40 \%$. Hence, while we would expect to detect the difference when comparing a large number of fibres, it is possible that our limited sample size explains our inability to detect the fibre type difference. Indeed, due to the time-consuming nature of the analysis, an average of 5-6 fibres was analysed per biopsy sample. Considering the coefficient of variations involved (10-15\%), and the expected difference in glycogen concentration as determined biochemically $(17-40 \%)$, it is likely that the limited sample of fibres per individual is responsible for our inability to detect the fibre type difference.

The explanations for the smaller glycogen particles in type I fibres at resting glycogen concentration may reside, at least partly, in the number of glycogen particles. 
While the type I fibres were found to have smaller particles, they were also found to have 18 and $25 \%$ more glycogen particles than type IIA and IIB fibres, respectively (Chapter V). While these differences did not reach statistical significance due to the big standard deviations associated with those measurements, it is interesting that these findings are in agreement with the recently reported fibre type differences in glycogenin activity and protein expression. Glycogenin activity and protein expression were recently shown to vary according to fibre types in rats (Hansen et al. 2000). The ranked order was, for both protein expression and activity, $\mathrm{I}>\|\mathrm{A}>\| \mathrm{IB}$. Considering that overexpressing glycogenin was recently shown to cause a production of more, smaller, glycogen particles (Roach et al. 1997), the fibre type dependency on particle number found in the present study could be more than just a coincidence. In other words, the fact that the type I fibres express more glycogenin could explain their greater number of smaller glycogen particles. However, while these hypotheses are interesting, one should consider that this comparison of fibre type differences in glycogenin activity was performed in rat skeletal muscle. Since it has been established that the fibre type differences are generally more marked in rat than human skeletal muscles, these observations are only speculative.

An alternate explanation for the ranked order of glycogen particle size (IIB > IIA > 1) consistently found in resting samples may relate to the metabolic demand imposed upon each fibre type. It is interesting that this order represents the natural order of fibre type recruitment during voluntary muscle contraction (Henneman, 1957). Hence, one could speculate that since type I fibres are being constantly recruited during activities of daily living, their glycogen particles are in a more dynamic state of turnover, and do not get a chance to enlarge to the same extent as type IIA and IIB glycogen particles. The same phenomenon could also explain the $13 \%$ greater particle volume in type IIB compared to type IIA. 
The last fibre type difference observed in the subcellular distribution of glycogen was that type I fibres, compared to both type IIA and IIB, had a significantly greater proportion of their myofibrillar glycogen located in their intra-myofibrillar space. This finding is in agreement with those of Schmalbruch \& Kamieniecka (1974) in human subjects, who found a significant proportion of the glycogen in type I to be located between the actin and myosin filaments, and the glycogen in type IIA and IIB to be preferentially distributed between myofibrils. If we relate this finding to the known glycogen-SR complex discussed earlier, this fibre type difference could be related to the fact that type I fibres are known to have a less developed SR (Ogata and Yamasaki 1985). Indeed, in their examination of the three-dimensional structure of the SR in the three fibre types, Ogata and Yamasaki (1985) found the SR to be sparse on the surface of mitochondria, implying that the type I fibres have a much smaller total SR volume than the type II fibres. This could, by a yet unknown mechanism, force the glycogen particles to deposit more in the intra-myofibrillar space, where the SR is probably less involved in glycogen synthesis and distribution.

\section{Glycogen metabolism during exercise.}

Perhaps one of the most interesting findings of the present thesis relates to the pattern of glycogen metabolism during exercise (Chapter VII). As discussed in Chapter VII, the performance of a $2 \times 90$-second sprint exercise led to a significant decrease in myofibrillar glycogen concentration. This glycogen degradation was caused by a nonsignificant decrease in the average glycogen particle volume and number. To better understand the source of glucosyl units used during exercise, we plotted the absolute and relative frequency distribution of glycogen particle diameters (Chapter VII - Figure 35). We believe these to be complementary in obtaining a thorough understanding of the mechanisms responsible for the glycogen degradation. Briefly, the results presented 
in Figure 35 of Chapter VII can be summarised as follows: 1) the pre-to post-exercise changes in absolute frequency distribution of glycogen particle diameter demonstrates a downward shift throughout all ranges of glycogen particle diameter, albeit more marked in the higher range of particle diameters (> $25 \mathrm{~nm})$, and 2) the pre- to post-exercise changes in relative frequency distribution of particle diameter, which eliminates the potential effect of exercise on particle number, demonstrate no change in particles smaller than $22 \mathrm{~nm}$, an increase of particles between 22 and $32 \mathrm{~nm}$, and a decrease of particles larger than $32 \mathrm{~nm}$. However, the interpretation of these results is complex, and brings about a series of speculations. Hence, the following is an attempt at explaining the physiological mechanism involved in glycogen degradation during that type of intense exercise, taking into account that the average number and size of glycogen particles was not significantly different pre- and post-exercise.

Glycogen synthesis has consistently been demonstrated to be up-regulated in working skeletal muscles (Blom et al. 1986; Constable et al. 1984; Nielsen et al. 2001; Price et al. 1991; Vollestad et al. 1989). Hence, it should be emphasised that a downward shift in the frequency distribution of particles of a given diameter could in theory be explained by both, the synthesis of these particles into larger ones, or their degradation into smaller ones. Alternatively, the synthesis of smaller particles or the degradation of larger ones could cause an upward shift in the frequency distribution of particles of a given diameter.

That being said, the pre- to post-exercise relative increase in frequency distribution of intermediate sized particles could be explained by four main mechanisms. A relative increase in synthesis of particles smaller than $22 \mathrm{~nm}$ into larger particles, as well as the relative increase in degradation of particles larger than $32 \mathrm{~nm}$ into smaller particles, are all likely to have contributed to the relative accumulation in particles of intermediate diameter (22 to $32 \mathrm{~nm}$ ) observed in Chapter VII. Furthermore, a relative 
decrease in synthesis of the intermediate-size particles into larger ones and / or a relative decrease in their degradation into smaller ones might also cause their relative accumulation. Our TEM-based technique does not allow determining which of the above mentioned phenomena is responsible for the relative increase in particles of intermediate diameter (22 to $32 \mathrm{~nm}$ ) (Chapter VII - Figure 35 (B)). Figure 39 (A) illustrates these possibilities.

Figure 39 (A).

1
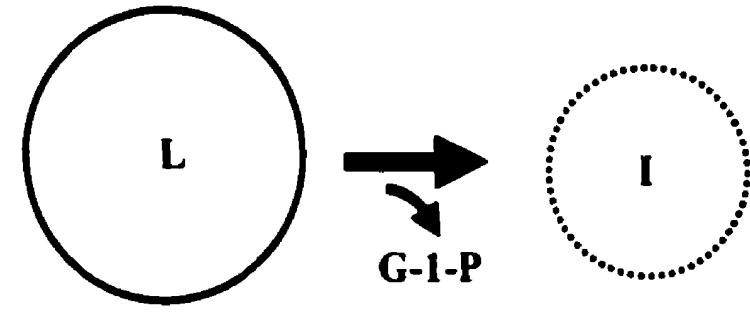

2
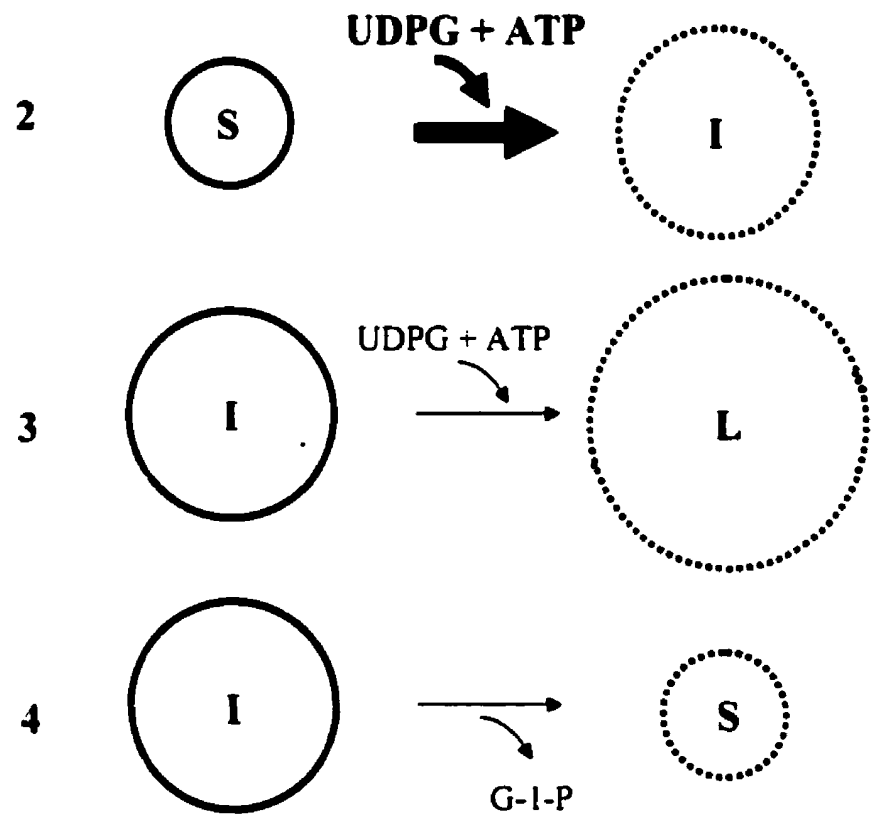

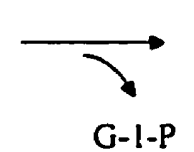

Figure 39 (A).

Illustrations of the four possible mechanisms to explain the relative increase in frequency distribution of intermediate-size glycogen particles. Wide arrows illustrate a relative increase in flux. Thin arrows represent a relative decrease in flux. Full circles represent the particle before its metabolism. Dashed circle represents the particle resulting from its metabolism.

$L=$ Larger particles $(>32 \eta \mathrm{m}$ or $>20000$ glucosyl units) $\mathrm{I}=$ Intermediate particles $(22-32 \mathrm{~nm}$ or $6000-20000$ glucosyl units) $S=$ Smaller particles $(<22 \eta \mathrm{m}$ or $<6000$ glucosyl units) G-1-P = Glucose-1-phosphate UDPG = uridine diphosphate-glucose 
On the other hand, the decrease in both the absolute and relative frequency distribution of particles larger than $32 \mathrm{~nm}$ clearly implies an increase in net degradation of these larger particles. However, the exercise-induced increase in degradation of glycogen particles greater than $32 \mathrm{~nm}$, as well as the decreased synthesis of particles smaller than $32 \mathrm{~nm}$ into larger ones, could all contribute to the decreased frequency of these largest particles. Figure 39 (B) illustrates these possibilities.

Figure 39 (B).

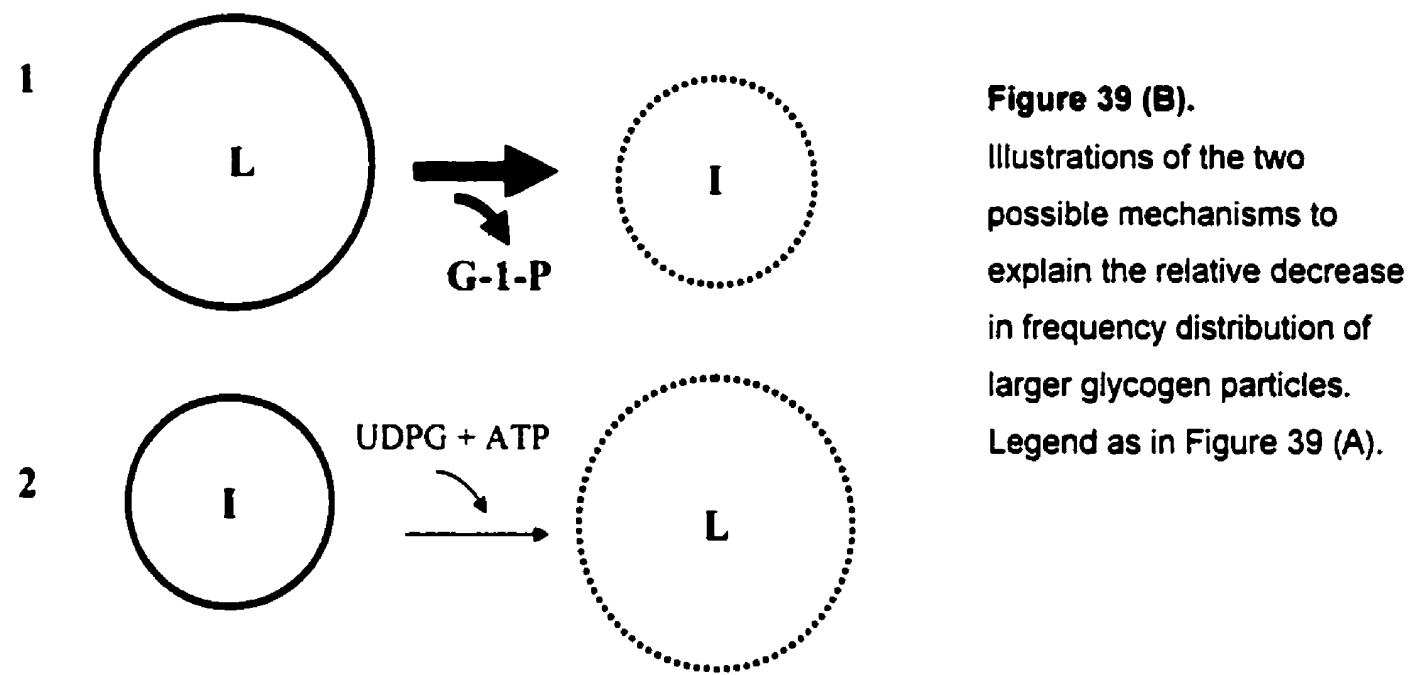

The reason why the relative frequency distribution of the smallest glycogen particles (<22 nm) was not affected by exercise, despite a small downward shift in absolute frequency, is unclear. This implies that, despite the small absolute decrease in the number of particles smaller than $\sim 22 \mathrm{~nm}$, the same proportion of particles had a diameter below $\sim 22 \mathrm{~nm}$ pre- and post-exercise. For this to happen, the sum of the mechanisms that are responsible for an increase in frequency (Figure $39(C)(a)$ ) and 
those that are responsible for a decrease in frequency (Figure 39 (C) (b)) must be equal during the exercise.

Given the preferential degradation of larger glycogen particles, combined with the relative accumulation observed in the frequency distribution of intermediate-size particles (Chapter VII - Figure 35), we would have expected an even greater relative increase in the frequency distribution of these smaller particles. It is possible that these particles are being formed by the simple degradation of larger particles (Figure $39(B)$ ), while an equivalent amount of these smaller particles are being degraded to undetectable levels (Figure 39 (C) (b)), thus keeping the relative frequency distribution unchanged. However, this is not likely considering the relative accumulation on particles of intermediate sizes. Alternatively, it is possible that these smaller particles are being synthesised into intermediate-size particles while other mechanisms are causing an equivalent increase in the frequency of these smaller particles, thus keeping the relative frequency similar pre- and post-exercise. The mechanisms that could potentially cause a relative increase in the frequency of these smaller particles include the degradation of intermediate size particles, as well as the synthesis of new particles from glycogenin (Figure 39 (C) (a)). If new particles were being synthesised from glycogenin, it would simply "push" the particles rightward in the distribution, and contributing to the relative increase in particles ranging from 22 to $32 \mathrm{~nm}$ in diameter. As mentioned above, this would add to the leftward shift of larger glycogen particles caused by their degradation, and cause a relative net glycogen synthesis of particles ranging from 22 to $32 \mathrm{~nm}$. However, the origin of the glycogenin required to synthesise new glycogen particles is unclear. While previous studies have shown that no deglucosylated glycogenin exists under most normal conditions in skeletal muscle, intense electrical stimulation depleting $85 \%$ of skeletal muscle glycogen has been demonstrated to release free glycogenin and GS (Smythe et al. 1990). Similarly, acute administration of adrenaline, under 
conditions that result in complete degradation of about $50 \%$ of the glycogen particles, has been reported to give rise to glycogen-free glycogenin in skeletal muscles (Lomako et al. 1990). Hence, it is possible that the glycogen depletion produced by the intense exercise in the present study caused the release of some free glycogenin, which could potentially be re-utilised to synthesise new particles, thus preventing a downward shift of the frequency distribution in that smaller range of diameter. Whether it is this glycogenin recycling or the formation of new glycogenin in vivo during the exercise, or both, that would explain this hypothetical formation of new glycogen particles during intense exercise is unknown.

Another possibility is that, due to the interactions of carbohydrates and enzymes in such small particles, no net glycogen degradation normally occurs in particles of that size, only net glycogen synthesis. This would explain why deglucosylated-glycogenin is not normally observed in skeletal muscles (Smythe et al. 1990). Hence, while the degradation of larger particles should lead to an accumulation of smaller ones during exercise, the net synthesis of the existing smaller particles $(<22 \mathrm{~nm})$ into larger ones would prevent the relative accumulation of glycogen particles in that range of the distribution. Furthermore, assuming that the stimulus for net glycogen synthesis is stronger in the smallest of these glycogen particles (Marchand et al. 2001), that the stimulus for synthesis and degradation are relatively equal in the intermediate-sized particles (between 22 and $32 \mathrm{~nm}$ ), and that of net degradation is stronger in the largest particles (>32 nm), that would explain the relative accumulation of particles in the intermediate size range. Figure 39 (C) summarises the possibilities. 
Figure 39 (C).

Figure 39 (C).

(a) Mechanisms to increase the relative frequency of smaller particles:

Illustrations of the possible mechanisms<smiles>c1ccccc1</smiles><smiles>C1C2CC1C2</smiles>
UDPG + ATP<smiles>c1ccccc1</smiles>

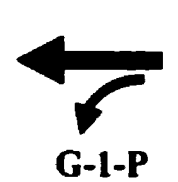

G-1-P

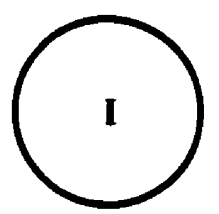

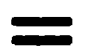

(b) Mechanisms to decrease the relative frequency of smaller particles:<smiles>c1ccccc1</smiles>

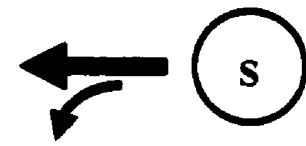

G-1-P

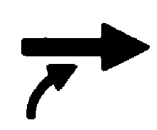

UDPG + ATP

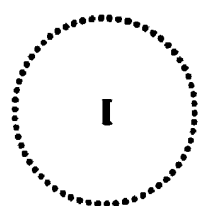

to explain the lack of change in the relative frequency distribution of smaller glycogen particles. The sum of mechanisms to increase and decrease the frequency must be equal during the exercise.

Legend as Figure 39

(A).

Taken together, the mechanisms most likely responsible for explaining the changes that occurred in the relative frequency distribution of all particle size ranges are illustrated in Figure 39 (D). 
Figure 39 (D).

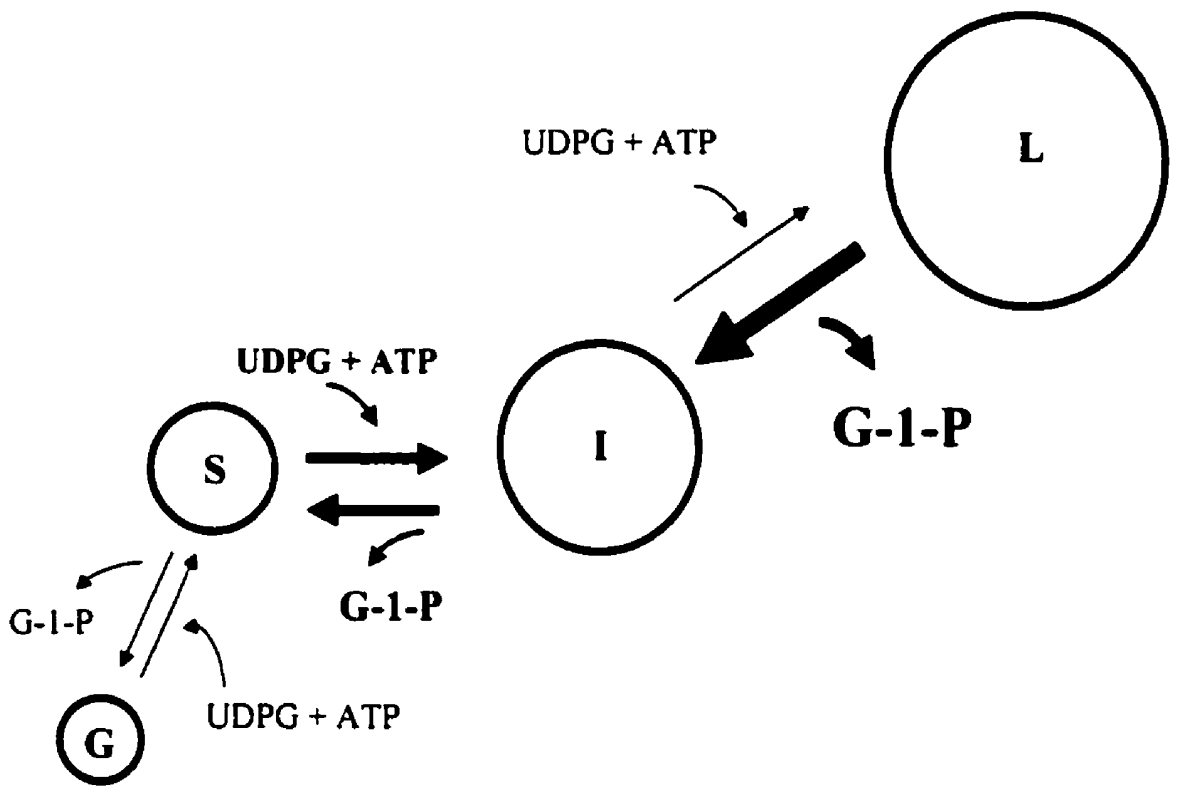

Figure 39 (D). Sum of mechanisms most likely involved in the pre-to post-exercise changes in relative frequency distribution reported in Chapter VII. Legend as in Figure 39 (A).

Alternatively, the findings could simply by explained by the combination of 1) a significant degradation of the larger particles; 2) a less significant degradation of the intermediate and smaller particles; and 3) a significant increase in the pool of G-6-P which would lead to the immediate re-incorporation of the glucose into glycogen particles. The rate of incorporation would be dictated by factors such as the number of particles around, the GS activity of the particle and the particle location. Based on the number of the smaller and intermediate particles relative to the larger ones, they should be able to re-incorporate more G6P compared to the larger ones. Hence, this would contribute to the more significant decrease in size of larger particles compared to the smaller and intermediate ones. 
Regardless of the physiological explanation for the results discussed above, we present the first direct evidence to suggest that it is the largest glycogen particles that are preferentially degraded during intense exercise. This is clearly demonstrated in Figure 40 , which plots the pre- to post-exercise $\%$ of decrease in absolute particle number for each particle diameter (data from Chapter VII). This figure also highlights a greater tendency for the smaller particles to undergo a relative increase as opposed to decrease in frequency. This would tend to argue in favour of the relative synthesis of the glycogen particles in the smaller size range. A preliminary statistical analysis comparing the percentage of decrease per range of glycogen particle diameter showed that the \% decrease was significantly increased with increasing particle size $(P>0.001)$.

Figure 40.

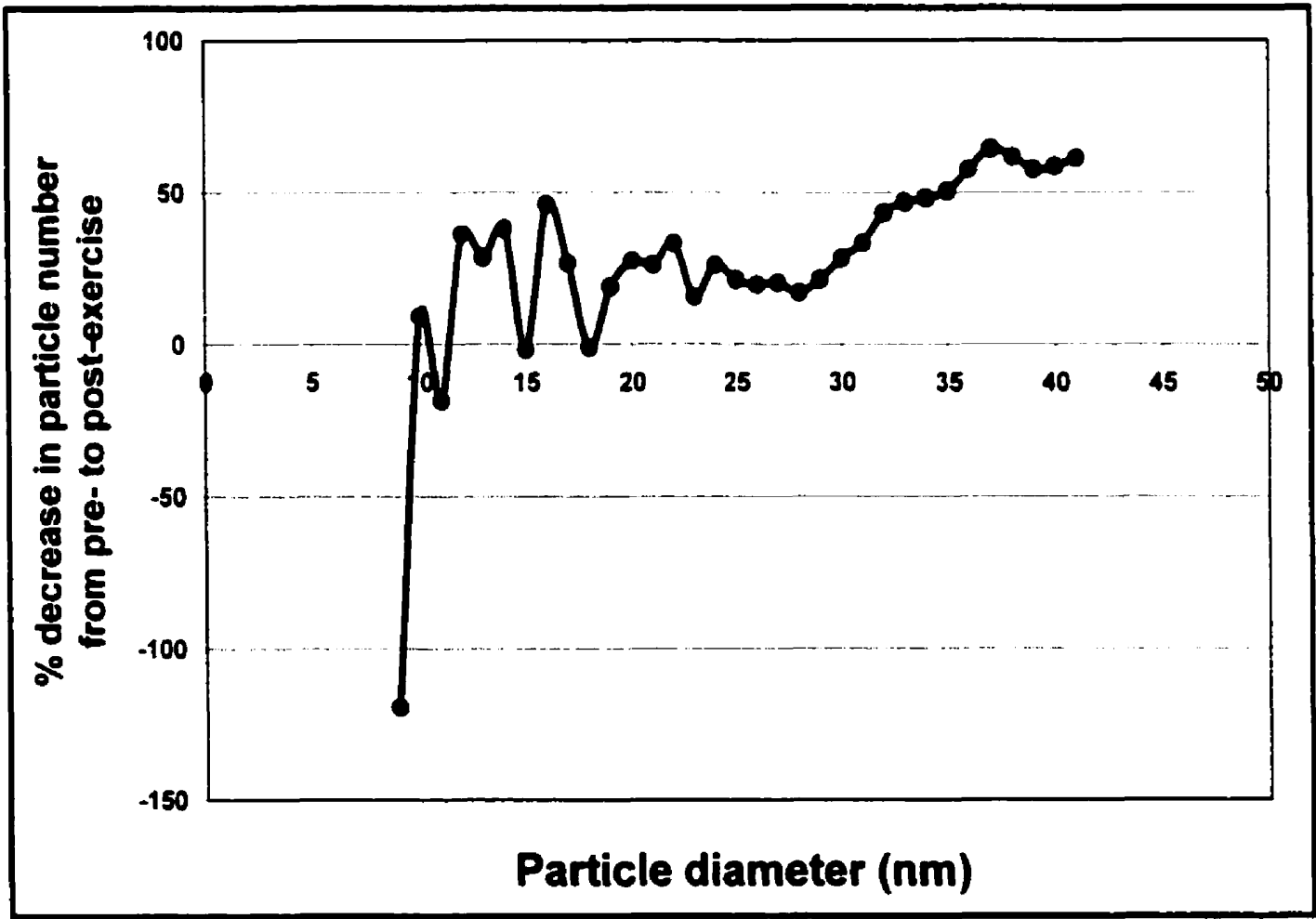


The results presented here may explain the lack of significant effect of exercise on the overall particle volume and number. Since the statistical procedure involves comparing the pre- and post-exercise average particle number and volume, it is possible that the selective decrease in volume and number of larger glycogen particles was not detected statistically on the basis that those particles represent a very small proportion of the total muscle glycogen particles. As discussed above, this is related to the concept of increasing glucosyl unit density as particle diameter increases. Hence, since the particles mainly degraded were specifically those of greater size, the density of glucosyl units per tier removed is very important. Therefore, the degradation of a small number of large glycogen particles represents a large amount of glucosyl units. Together, these data provide strong evidence to show that the larger glycogen particles are degraded to a greater extent than the smaller ones during intense exercise.

Whereas the above interpretation of a possible net synthesis of smaller glycogen particles during exercise may seem paradoxical, the existence of a constant glycogen turnover in skeletal muscle has been repeatedly demonstrated, although not in such an intense exercise. This cycling of skeletal muscle glycogen has been observed at rest (Cline et al. 1997), as well as during periods of net glycogen synthesis (Laurent et al. 2000) and breakdown (Constable et al. 1984; Huang 1998; Hutber and Bonen 1989; Nielsen et al. 2001; Price et al. 1991 and 1994). Furthermore, as mentioned above, the up-regulation of glycogen synthesis in working skeletal muscles has been shown in many experiments (Blom et al. 1986; Constable et al. 1984; Nielsen et al. 2001; Price et al. 1991; Vollestad et al. 1989). Given the intense nature of the metabolic demand during exercise, one would expect that during contractions, all available glucose and ATP would be directed towards providing energy to fuel the contractions instead of being utilised as substrates for glycogen storage. However, Nielsen and colleagues (2001) 
suggested that the up-regulation of glycogen synthesis during exercise might be part of a functional mechanism of glycogen sparing in the exercising muscle, protecting it against complete glycogen depletion during intense exercise or early glycogen depletion during prolonged exercise. Furthermore, the clear advantage of an increased glycogen synthase activity during exercise is to prime the muscle cell for glycogen synthesis immediately at the cessation of exercise (Nielsen et al. 2001). Based on our findings and the above discussion, the lack of changes in the relative frequency distribution of the smaller glycogen particles, combined with the relative accumulation of those of an intermediate size and degradation of the larger ones, support that a constant turnover also occurs during intense exercise, and that the size of the individual particles is likely to dictate their behaviour.

This concept of the importance of the size of an individual glycogen particle in controlling its own metabolism is also supported by the results presented in Chapter VI. As discussed, it is the size of a given glycogen particle, rather than the glycogen concentration in its compartment, which appears to control its rate of synthesis. Based on those results, we hypothesised that the size of individual glycogen particle could alter the interactions with the metabolising enzymes. This in turn would favour a net glycogen synthesis or degradation, depending on the interactions dictated by the size of the glycogen particle.

This concept has been previously suggested by Roach and Skurat (1997). They proposed that the composition of the glycogen-protein complex is likely to be dynamic. and suggested that during the early stages of synthesis, binding of degradative enzymes like GP might not be favoured, thus allowing the formation of small glycogen molecules even in the face of active GP. In agreement with this, it has been suggested previously that the $K_{m}$ for glucose is smaller for the GS associated with PG than for the GS 
associated with MG (Curtino et al. 2000; Lomako et al. 1993). As stated earlier, we believe that PG and MG simply represent the smaller and larger ranges of glycogen particles, respectively. Accordingly, while we do not think that two distinct GS pools actually exist, we do believe that the difference in GS activity for glycogen particles of different sizes reported by Curtino and colleagues (2000) and Lomako and co-workers (1993) reflect the effect of glycosome size on enzymatic activity.

The intra-muscular glycogen concentration has consistently been demonstrated to exert a powerful regulatory effect on glycogen synthesis (Laurent et al. 2000; Montell et al. 1999; Munger et al. 1993; Price et al. 2000; Vandenberghe et al. 1999). However, limited information is available regarding the mechanisms by which glycogen levels feedback to regulate glucose transport and glycogen metabolism. In a recent experiment, Nielsen and colleagues (2001) provided evidence to show that GS localisation is influenced by muscle glycogen concentration. In addition, they demonstrated that glycogen concentration was a far more potent regulator of GS than insulin, and that the well-known stimulatory effect of muscle contraction on GS activity is solely a function of the ability of contractions to decrease muscle glycogen concentration (Nielsen et al. 2001). Furthermore, it was recently demonstrated that insulin-stimulated glycogen synthesis in human skeletal muscle is also negatively influenced by glycogen content (Laurent et al. 2000), supporting the notion that glycogen inhibits its own synthesis. Other recent evidence also supports the fact that high muscle glycogen levels promote glycogen phosphorylase (GP) activity (Hespel and Richter 1992; Shearer et al. 2001; Vanderberghe et al. 1999). Considering the results presented in this thesis, and given that glycogen concentration correlates almost perfectly with the size of individual glycogen particles (Chapter IV - Detailed Methods), we speculate that the influence of glycogen concentration on the control of glycogen metabolism operates at 
the level of the individual glycogen particles. Accordingly, altering the size of the glycosomes would affect the activity of the different enzymes, hence controlling the synthesis and degradation of skeletal muscle glycogen.

Several mechanisms involved in the control of the different enzymes are likely to be affected by the size of the glycogen particles. The physical association of the enzymes with the glycosomes is probably one of the most important ones. Accordingly, differences in GS activity or susceptibility to activation when bound or unbound to glycogen have been hypothesised (Lane et al. 1989). Alternatively, it has been proposed that the activity of GS in vitro is high only when glycogenin and GS are bound to each other (Pitcher et al. 1987 and 1988). This could explain why GS activity decreases as the glycogen particle grows (Nielsen et al. 2001). While changes in the association of the glycosomes with GS and GP themselves is likely, different associations of the kinases and phosphatases involved in controlling their phosphorylation state is also credible.

Accordingly, an important concept is the recent evidence that has shown the family of glycogen targeting subunits of protein phosphatase-1 to play a major role in the co-ordination of glycogen metabolism (Newgard et al. 2000). These targeting sub-units have not only been shown to target the catalytic subunit of PP1 to the glycogen particle, but also to bind differentialiy to GS, GP and phosphorylase kinase (Printen et al. 1997). These targeting proteins have been said to serve as a "molecular scaffolds" by juxtaposing the enzymes with its substrates in a macromolecular complex and, in the process, to exert profound effects on PP1 activity and glycogen metabolism (Newgard et al. 2000). Hence, the relation between enzymatic activity and glycogen levels, and consequently glycogen particle size, might be regulated by association-dissociation of proteins in the glycosome. In line with this hypothesis, it has been previously proposed 
that as the glycogen particle decreases in size, GS and PP1 are both released from the glycogen particle and become available for activation (Villar-Palasi 1969). Furthermore, it has recently been shown that the binding of GS to the glycogen targeting subunits of PP1 is apparently important for the activity of GS (Newgard et al. 2000). However, the extent to which glycogen concentration affects the binding of GS to the regulatory subunit of PP1 is not known (Newgards et al. 2000). Taken together, these findings indeed suggest a role for the targeting subunits of PP1 in regulating the glycogen metabolism at the individual glycosome level.

Over the recent years, there has been an increased focus on the significance of spatial compartmentalisation of the enzymes involved in glycogen metabolism. Indeed, the translocation of GLUT-4 from an intracellular pool to the plasma membrane in response to insulin stimulation (Lund 1993) or muscular contractions (Cartee et al. 1989) is well established. Similarly, the liver equivalent of hexokinase II, glucokinase, has also been shown to translocate between the cytoplasm and the nucleus in response to changes in glucose concentration, hence affecting its inhibition by regulatory proteins (Brown 1997). A similar phenomenon of translocation to different subcellular locations in various tissues has recently been observed for glycogen synthase (GS) in response to diverse stimuli (Brady et al. 1999; Fernandez-Novell et al. 1997; Garcia-Rocha et al. 2001; Jensen et al. 2000; Skurat et al. 1997). In a recent report, the phenomenon of GS translocation was observed in rat skeletal muscles (Nielsen et al. 2001). Nielsen and coworkers (2001) found that GS translocates from a glycogen-enriched membrane fraction to a cytoskeleton fraction when glycogen levels are decreased, and hence, that GS subcellular location is influenced by muscle glycogen content. In that experiment demonstrating the glycogen concentration dependent intracellular translocation of GS in 
rat skeletal muscles, Nielsen and colleagues (2001) suggested the possibility that the targeting subunits of PP1 might be involved in the GS translocation process. They proposed that one possible way in which glycogen concentration might affect GS activity could be through interaction with the glycogen targeting subunits of PP1, which bind the catalytic subunit of PP1 to glycogen and also bind GS, GP and phosphorylase kinase. It is unknown whether a similar subcellular translocation phenomenon is also involved in the control of GP. Furthermore, it is unclear whether only GS translocates or whether the entire glycosome is also re-localised. Hence, further studies are required to clarify these important research questions.

The subcellular localisation of the glycosome may have important physiological implications. For example, the faster glycogen synthesis in the intra-myofibrillar location observed in the present study, in close proximity to the contractile apparatus, could simply constitute a high priority. When glycogen stores near the contractile apparatus are filled up, then further glycogen synthesis can occur in other regions of the cell and GS association with the myofibrils is relaxed (Nielsen et al. 2001). Alternatively, the subcellular location of the glycogen particles is likely to alter the metabolic environment, including its susceptibility to hormones, as well as the availability of different substrates and allosteric regulators.

\section{Limitations and future considerations.}

The method developed and presented in this thesis provides the unique ability to quantify the distribution of skeletal muscle glycogen with respect to glycogen particle size, number and different subcellular compartments. A number of assumptions were presented and discussed earlier. Most important is our assumption that the glycogen volume in each image has a maximal thickness equal to the average particle diameter. While this issue was addressed in the section "Method validity and reliability" above, our 
inability to visualise, and hence to quantify, the particles pilled up on top of each other in a given thin section certainly represents an important limitation of our method. In addition, it should be emphasised that while this technique provides the ability to quantify total skeletal muscle glycogen concentration, the technique is not designed to replace standard biochemical methods for measuring skeletal muscle glycogen. The tedious nature of the analysis makes it an effective but inefficient technique for measuring total muscle glycogen concentration. Hence, this technique should be used as a complement to standard biochemical methods. The time consuming-nature of the analysis introduces an important limitation of the present thesis with regards to the number of fibres analysed for each biopsy sample (5-6 fibres). This, in turn, limits the sample size that can realistically be included in every experiment, and is most likely responsible for our inability to detect fibre type differences in total glycogen concentration, and potentially, other physiologically important differences. It is likely that the development of a more advanced technology in the field of TEM and image analysis will increase the efficiency of the technique, and therefore decrease the significance of this limitation.

The advancing technology in TEM, direct digitising of images, in vivo visualisation of samples and more specific contrasting technique will increase the efficiency and facilitate the use of TEM-based methods in studying glycogen metabolism. Further experiments are required to ascertain the physiological role of the glycogen in each specific compartment in skeletal muscle cellular functions. With the recent inclusion of subcellular compartmentalisation as an important control mechanism in glycogen metabolism, the specific role of the glycogen stored within each compartment represents essential information in order to achieve a complete understanding of the complex interactions involved. Furthermore, the comparison of the subcellular distribution of glycogen in different conditions, including insulin resistance (i.e. obesity, diabetes, etc.), glycogen storage diseases, and other pathological states related to a defect in glycogen 
metabolism, will provide invaluable information to help elucidate the mechanistic defects involved. It will be interesting to examine the specific relation between the insulindependent and insulin-independent phases of glycogen synthesis with the specific pattern of increase in glycogen particle number and size. This could have important implications for the treatment of diabetes mellitus and other insulin-resistant states. Finally, the use of the immuno-gold technique to label different enzymes and proteins involved in glycogen metabolism, and their visualisation with TEM will provide unique information about the association of the glycogen-enzyme complex and its subcellular distribution within the skeletal muscle cell.

\section{Conclusion.}

The ultrastructural analyses of human skeletal muscle glycogen reported in this thesis provided the first qualitative data on glycogen metabolism. The data presented constitute essentially novel information concerning a compartmentalised pattern of glycogen distribution and metabolism. These data are in line with the emerging biochemically-based concept of the subcellular organisation of the enzymatic machinery and metabolic pathways into an integrated compartmentalised glycogen metabolism. To conclude, the existence of the glycogen particles as a structure in or by which various specific functions are carried out defines it as a cellular organelle named glycosomes (Rybicka 1996). As consistently demonstrated in the literature, the long-identified skeletal muscle glycogen $\beta$-particle (Wanson and Drochmans 1968) not only represents the glucose storage form of skeletal muscle glycogen, but also represents a complete enzymatic machinery with protein to protein interactions which allows it to interact with various cellular components and to control carbohydrate metabolism. It is becoming apparent that these assemblies are likely to be dynamic, and that not all the interactions involved within the glycogen particle need to be permanent. Therefore, different 
assemblages may prevail at different times and in different subcellular compartments, changing with the metabolic state of the cell. Further research in this area is required in order to provide a complete understanding of these complex interactions. 


\section{References}


Adamo, K.B., and T.E. Graham. (1998a). Comparison of traditional measurements with macroglycogen and proglycogen analysis of muscle glycogen. Journal of Applied Physiology. 84: 908-913.

Adamo, K.B., M.A. Tarnopolsky, and T.E. Graham. (1998b). Dietary carbohydrate and post-exercise synthesis of proglycogen and macroglycogen in human skeletal muscle. American Journal of Physiology (Endocrinology and Metabolism). 275: E229-E234.

Afonso, A., Macedo, P.M., Ellis, A.E. and Silva, M.T. (2000). Glycogen granules in resting and inflammatory rainbow trout phagocytes - an ultrastructural study. Diseases of aquatic organisms. 42: 101-110.

Alonso, M.D., Lomako, J., Lomako, W.M. and Whelan, W. (1995). A new look at the biogenesis of glycogen. FASEB Journal . 9: 1126-1137.

Asp, S., Daugaard, J.R., Rohde, T., Adamo, K. and Graham, T. (1999). Muscle glycogen accumulation after a marathon: roles of fiber type and pro- and macroglycogen. Journal of Applied Physiology. 86: 474-478.

Azpiazu, I., Manchester, J., Skurat, A.V., Roach, P.J. and Lawrence, J.C.Jr. (2000). Control of glycogen synthesis is shared between glucose transport and glycogen synthase in skeletal muscle fibers. American Journal of Physiology (Endocrinology and Metabolism). 278: E234-E243.

Bangsbo, J., Gollnick, P.D., Graham, T.E. and Saltin, B. (1991). Substrates for muscle glycogen synthesis in recovery from intense exercise in man. Journal of Physiology 
(London). 451: 205-227.

Banqué, S., Guinovart, J.J. and Ferrer, J.C. (1997). Glycogenin, the primer of glycogen synthesis, binds to actin. FEBS letters. $417: 355-359$.

Barstow, T.J., P.H. Nguyen, and R. Casaburi. (1996). Influence of muscle fibre type and pedal frequency on oxygen uptake kinetics of heavy exercise. Journal of Applied Physiology. 81: 1642-1650.

Bergmeyer, H. (1974). Methods of Enzymatic Analysis. New York: Academic, vol. 3, p. 1128-1135.

Biava, C. (1963). Identification and structural forms of human particulate glycogen. Laboratory Investigation. 12: 1179-1197.

Blom, P.C.S., Vollestad, N.K. and Costill, D.L. (1986). Factors affecting changes in muscle glycogen concentration during and after prolonged exercise. Acta Physiologica Scandinavica. 128 (Supplement 556): 67-74.

Bogdanis, G.C., Nevill, M.E., Boobis, L.H. and Lakomy, H.K.A. (1996). Contribution of phosphocreatine and aerobic metabolism to energy supply during repeated sprint exercise. Journal of Applied Physiology. 80: 876-884.

Bouchard, C., J.A. Simoneau, G. Lortie, M.R. Boulay, M. Marcotte, and M.C. Thibault. (1986). Genetic effects in human skeletal muscle fiber type distribution and enzyme activities. Canadian Joumal of Physiology and Pharmacology. 64: 1245-1251. 
Brady, M.J., Kartha, P.M., Aysola, A.A. and Saltiel, A.R. (1999). The role of glucose metabolites in the activation and translocation of glycogen synthase by insulin in 3T3-L1 adipocytes. The Journal of Biological Chemistry. 274: 27497-27504.

Brown, K.S., Kalinowski, S.S., Megill, J.R. Durham, S.K. and Mookhtiar, K.A. (1997). Glucokinase regulatory protein may interact with glucokinase in the hepatocyte nucleus. Diabetes. 46: 179-186.

Bulloch, R.T., Pearce, M.B., Murphy, M.L., Jenkins, B.J. and Davis, J.L. (1972). Myocardial lesions in idiopathic and alcoholic cardiomyopathy. Study by ventricular septal biopsy. The American Journal of Cardiology. 29: 15-25.

Busby, S.J.W. and Radda, G.K. (1976). Regulation of the glycogen phosphorylase system: from physical measurements to biological speculations. Current Topics in Cellular Regulation. 10: 89-160.

Cadefeau, J. Bollen, M. and Stalmans, W. (1997). Glucose-induced glycogenesis in the liver involves the glucose-6-phosphate-dependent dephosphorylation of glycogen synthase. Biochemical Journal. 322: 745-750.

Calder, P.C. and Geddes, R. (1985). Glycogen of high molecular weight from mammalian muscle. Carbohydrate Research. 135: 249-256.

Cao, Y., Skurat, A.V., DePaoli-Roach, A.A. and Roach, P.J. (1993). Initiation of glycogen synthesis. Control of glycogenin by glycogen phosphorylase. The Journal of Biological chemistry. 268: 21717-21721. 
Cartee, G.D., Young, D.A., Sleeper, M.D., Zierath, J., Wallberg-Henriksson, H. and Hollowsky, J.O. (1989). Prolonged increase in insulin-stimulated glucose transport in muscle after exercise. American Joumal of Physiology (Endocrinology and Metabolism). 256: E494-E499.

Casey, A., Short, A.H., Hultman, E. and Greehaff, P.L. (1995). Glycogen resynthesis in human muscle fibre types following exercise-induced glycogen depletion. Journal of Physiology. 483: 265-271.

Caudwell, F.B. and Cohen, P. (1980). Purification and subunit structure of glycogenbranching enzyme from rabbit skeletal muscle. European Journal of Biochemistry. 109: 391-394.

Chasiotis, S. Sahlin, K. and Hultman, E. (1982). Regulation of glycogenolysis in human muscle at rest and during exercise. Journal of Applied Physiology (Resp. Environ. Exercise Physiol.). 53: 708-715.

Clarke, F.M., Stephan, P., Huxham, G., Hamilton, D. and Morton, D.J. (1984). Metabolic dependence of glycolytic enzyme binding in rat and sheep heart. European Journal of Biochemistry. 138: 643-649.

Cline, G.W., Magnusson, I., Rothman, S.L., Petersen, K.F., Laurent, D. and Shulman, G.I. (1997). Mechanism of impaired insulin-stimulated muscle glucose metabolism in subjects with insulin-dependent diabetes mellitus. Journal of Clinical Investigation. 99: 2219-2224. 
Coderre, L., Kandror, K.V., Vallega, G. and Pilch, P.F. (1995). Identification and characterization of an exercise-sensitive pool of glucose transporters in skeletal muscle. Journal of Biological Chemistry. 270: 27584-27588.

Cogswell, A.M., Stevens, R.J. and Hood, D.A. (1993). Properties of skeletal muscle mitochondria isolated from subsarcolemmal and inter-myofibrillar regions. American Journal of Physiology (Cell Physiology). 264: C383-C389.

Cohen, P. (1978). The role of cyclic-AMP-dependent protein kinase in the regulation of glycogen metabolism in mammalian skeletal muscle. Current Topics in Cellular Regulation. 14: 117-96.

Cohen, P. (1986). Muscle glycogen synthase. In: The enzymes ( $3^{\text {rd }}$ ed.), edited by P. Boyer and E.G. Krebs. Orlando, FL: Academic. 17: 461-497.

Cohen, P. (1989). The structure and regulation of protein phosphatases. Annual Review of Biochemistry. 58: 453-508.

Conlee, R.K., Hickson, R.C., Winder, W.W., Hagberg, J.M. and Holloszy, J.O. (1978). Regulation of glycogen synthesis in muscles of rats following exercise. American Journal of Physiology (Regulatory Integrative and Comparative Physiology). 4: R145. R150.

Connett, R.J. and Sahlin, K. (1996). Control of glycolysis and glycogen metabolism. in : Handbook of Physiology. Exercise: Regulation and integration of multiple systems (Section 12), edited by Rowell and Shepherd, p. 870-911. 
Constable, S.H., young, J.C., Higuchi, M. and Holloszy, J.O. (1984). Glycogen resynthesis in leg muscles of rats during exercise. American Journal of Physiology (Regulatory Integrative and Comparative Physiology). 247: R880-R883.

Curtino, J.A. and Lacoste, E.R. (2000). Two glycogen synthase activities associated with proteoglycogen in retina. Neurochemical Research. 25: 129-132.

Deibert, D.C. and DeFronzo, R.A. (1980). Epinephrine-induced insulin resistance in man. Journal of Clinical Investigation. 65: 717-721.

Derave, W., Gao, S. and Richter, E.A. (2000). Pro- and macroglycogenolysis in contracting rat skeletal muscle. Acta Physiologica Scandinavica. 169: 291-296.

Derave, W., Lund, S., Hoiman, G.D., Wohtaszewsky, J., Pedersen, O. and Richter, E.A. (1999). Contraction-stimulated muscle glucose transport and GLUT-4 surface content are dependent on glycogen content. American Journal of Physiology (Endocrinology and Metabolism). 277: E1103-E1110.

Devos, P., Baudhuin, P., Van Hoof, F. and Hers, H.G. (1983). The alpha particulate liver glycogen. A morphometric approach to the kinetics of its synthesis and degradation. Biochemical Journal. 209: 159-165.

Dohm, G.L. and Dudek, R.W. (1998). Role of transverse tubules (T-tubules) in muscle glucose transport. In: Skeletal Muscle Metabolism in Exercise and Diabetes, edited by Richter et al. Plenum Press, New-York, p. 27-34. 
Douen, A.G., Ramlal, T., Cartee, G.D. and Klip, A. (1990). Exercise modulates the insulin-induced translocation of glucose transporters in rat skeletal muscle. FEBS letters. 261: 256-260.

Drochmans, P. (1960) Mise en évidence du glycogène dans la cellule hépatique par microscopie électronique. Journal of Biophysical and Biochemical Cytology. 8: 553558.

Drochmans, P. (1962). Morphologie du glycogène. Etude au microscope électronique de colorations négatives du glycogène particulaire. Journal of Ultrastructructural Research. 6: 141-163.

Drochmans, P. (1963). Techniques for the isolation of particulate glycogen and its examination in the electron microscope. In: Biochemical Society. Symposia. 23: 127137.

Eisenberg, B.R. (1983). Quantitative ultrastructure of mammalian skeletal muscle. In: Handbook of Physiology (Section 10: Skeletal muscle). Edited by Peachey L.D. and R.H. Adrian. American Physiological Society, Bethesda, p.73.

Eisenberg, B.R. and Kuda, A.M. (1975). Stereological analysis of mammalian skeletal muscle. II. White vastus muscle of the adult guinea pig. Journal of Ultrastructure Research. 51: 176-187.

Eisenberg, B.R. and Kuda, A.M. (1976). Discrimination between fiber populations in mammalian skeletal muscle by using ultrastructural parameters. Journal of 
Ultrastructure Research. 54: 76-88.

Entman, M.L., Keslensky, S.S. Chu, A. and Van Winkle, W.B. (1980). The sarcoplasmic reticulum - glycogenolytic complex in mammalian fast twitch skeletal muscle. Proposed in vitro counterpart of the contraction-activated glycogenolytic pool. The Journal of Biological Chemistry. 255: 6245-6252.

Esbjornsson-Liljedahl, M., C.J. Sundberg, B. Norman, and E. Jansson. (1999). Metabolic response in type I and type II muscle fibers during a 30-s cycle sprint in men and women. Journal of Applied Physiology. 87: 1326-1332.

Essen, B. (1978). Glycogen depletion of different fibre types in human skeletal muscle during intermittent and continuous exercise. Acta Physiologica Scandinavica. 103: 446455.

Essen, B. and Henriksson, J. (1974). Glycogen content of individual muscle fibers in man. Acta Physiologica Scandinavica. 90: 645-647.

Evans, W.J., Phinney, S.D. and Young, V.R. (1982). Suction applied to a muscle biopsy maximizes sample size. Medicine and Sciences in Sports and Exercise. 14:101. 102.

Fell, R.S., McLane, J.A., Winder, W.W. and Holloszy. (1980). Preferential resynthesis of muscle glycogen in fasting rats after exhausting exercise. American Journal of Physiology (Regulatory Integrative and Comparative Physiology). 238: R328-R332. 
Fernandez-Novell, J.M., Bellido, D. Vilaro, S. and Guinovart, J.J. (1997). Glucose induces the translocation of glycogen synthase to the cell cortex in rat hepatocytes. Biochemical Journal. 321: 227-231.

Ferrans, V.J., Maron, B.J., Buja, L.M., Ali, N. and Roberts, W.C. (1975). Intranuclear glycogen deposits in human cardiac muscle cells: ultrastructure and cytochemistry. Journal of Molecular and Cellular Cardiology. 7: 373-386.

Friden, J., Serger, J. and Ekblom, B. (1985). Implementation of periodic acidthiosemicarbazide-silver proteinate staining for ultra-structural assessment of muscle glycogen utilization during exercise. Cell and Tissue Research. 242: 229-232.

Friden, J., Serger, J. and Ekblom, B. (1989). Topographical location of muscle glycogen: an ultrahistochemical study in the human vastus lateralis. Acta Physiologica Scandinavica. 135: 381-391.

Gaitanos, G.C., Williams, C., Boobis, L.H. and Brook, S. (1993). Human muscle metabolism during intermittent maximal exercise. Journal of Applied Physiology. 75: 712-719.

Garcia-Rocha, M., Roca, A., De La Isglesia, N., Bada, O., Fernandez-Novell, J.M., Ferrer, J.C. and Guinovart, J.J. (2001). Intracellular distribution of glycogen synthase and glycogen in primary cultured rat hepatocytes. Biochemical Journal. 357: 17-24.

Goldsmith, E., Sprang, S. \& Fletterick, J.R. (1982). Structure of Maltoheptaose by difference Fourier methods and a model for glycogen. Journal of Molecular Biology. 
$156,411-427$

Goldstein, M.A., Murphy, D.L., Van Winkle, W.B. \& Entman, M.L. (1985). Cytochemical studies of a glycogen-sarcoplasmic reticulum complex. Journal of Muscle Research and Cell Motility. 6: 177-187.

Gollnick, P.D., Karlsson, J., Piehl, K. and Saltin, B. (1974a). Selective glycogen depletion in skeletal muscle fibres of man following sustained contractions. Journal of Physiology. 241: 59-67.

Gollnick, P.D., Piehl, K. and Saltin, B. (1974b). Selective glycogen depletion pattern in human muscle fibers after exercise of varying intensity and at varying pedaling rates. Journal of Physiology. 241: 45-57.

Goodyear, L.J., Hirshman, M.F., Smith, R.J. and Horton, E.S. (1991). Glucose transporter number, activity, and isoform content in plasma membranes of red and white skeletal muscle. American Joumal of Physiology (Endocrinology and Metabolism). 261: E556-E561.

Graham, T.E., Adamo, K.B., Shearer, J., Marchand, I. and Saltin, B. (2001). Pro- and macro-glycogenolysis: relationship with exercise intensity and duration. Journal of Applied Physiology. 90: 873-879.

Greenhaff, P.L., Nevill, M.E., Soderlund, K., Bodin, K., Boobis, L.H., Williams, C. and Hultman, E. (1994). The metabolic responses of human type I and II muscle fibres during maximal treadmill sprinting. Journal of Physiology. 478: 149-155. 
Greenhaff, P.L., Ren, J.M., Soderlund, K. and Hultman, E. (1991). Energy metabolism in single human muscle fibers during contraction without and with epinephrine infusion. American Journal of Physiology (Endocrinology and Metabolism). 260: E713-E718.

Greenhaff, P.L., Soderlund, K., Ren, J.M. and Hultman, E. (1993). Energy metabolism in single human muscle fibres during intermittent contraction with occluded circulation. Journal of Physiology. 460: 443-453.

Gunja-Smith, Z., Marshall, J.J., Mercier, C., Smith, E.E. and Whelan, W.J. (1970). A revision of the Meyer-Bernfeld model of glycogen and amylopectin. FEBS letters. 12: 101-104.

Hansen, B.F. (1999). Limits to Glycogen Storage (Ph.D. thesis). Copenhagen: University of Copenhagen.

Hansen, B.F., Derave, W., Jensen, P. and Richter, E.A. (2000). No limiting role for glycogenin in determining maximal attainable glycogen levels in rat skeletal muscle. American Journal of Physiology (Endocrinology and Metabolism). 278: E398-E404.

Hansen, B.F., Jensen, P. and Barbetti, F. (1998). Glycogen storage in L6 cells is increased by overexpression of glycogenin. Diabetologia. 41: A201 (Abstract).

Harris, R.C., Essen, B. and Hultman, E. (1976). Glycogen phosphorylase activity in biopsy samples and single muscle fibres of muscle quadriceps femoris of man at rest. Scandinavian Journal of Clinical and laboratory Investigations. 36: 521-526. 
Hayat, M.A. (1989). Principles and techniques of electron microscopy In: Biological applications ( $3^{\text {rd }}$ edition). CRC press.

Henneman, E. (1957). Relation between size of neurons and their susceptibility to discharge. Science. 126: 1345-1347.

Henrikssen, E.J., Bourey, R.E., Rodnick, K.J., Koranyi, L., Permutt, M.A. and Holloszy, J.O. (1990). Glucose transporter protein content and glucose transport capacity in rat skeletal muscles. American Journal of Physiology (Endocrinology and Metabolism). 259: E593-E598.

Hespel, P. and Richter, E.A. (1992). Mechanism linking glycogen concentration and glycogenolytic rate in perfused contracting rat skeletal muscle. Biochemistry Journal. 284: $777-780$.

Himes, M.M. and Pollister, A.W. (1962). Symposium: synthetic processes in the cell nucleus. V. Glycogen accumulation in the nucleus. Jourmal of histochemistry and cytochemistry. 10: 175-185.

Hochachka, P.W. and Hulbert, W.C. (1977). Glycogen 'seas', glycogen bodies, and glycogen granules in heart and skeletal muscle of air-breathing, burrowing fish. Canadian Journal of Zoology. 56: 774-786.

Hubbard, M.J. and Cohen, P. (1997). On target with a new mechanism for the regulation of protein phosphorylation. Trends in Biochemical Sciences. 18: 172-177. 
Huang, M.T. (1998). Glycogen turnover in skeletal muscle is stimulated along with glucose uptake in vivo during contraction. Life Sciences. 63: 2023-3030.

Hultman, E. (1995). Fuel selection, muscle fiber. Proceedings of the Nutrition Society. 54: 107-121.

Hultman, E. (1967). Muscle glycogen in man determined in needle biopsy specimens. Scandinavian Journal of Clinical and Laboratory Investigation. 113: 337-340.

Hultman, E. and Bergstrom, J. (1967). Muscle glycogen in relation to diet studied in normal subjects. Acta Medica Scandinavica. 182: 109-117.

Hutber, C.A. and Bonen, A. (1989). Glycogenesis in muscle and liver during exercise. Journal of Applied Physiology. 66: 2811-2817.

Ivy, J.L. and Kuo, C.H. (1998). Regulation of GLUT-4 protein and glycogen synthase during muscle glycogen synthesis after exercise. Acta Physiologica Scandinavica. 162: 295-304.

James, D.E., Strube, M. and Mueckler, M. (1989). Molecular cloning and characterization of an insulin-regulatable glucose transporter. Nature. 338: 83-87.

Jansson, E., B. Sjodin, and P. Tesch. (1978). Changes in muscle fibre type distribution in man after physical training. Acta Physiologica Scandinavica. 104: 235-237. Jensen, T.C., Crosson, S.M., Kartha, P.M. and Brady, M.J. (2000). Specific desensitization of glycogen synthase activation by insulin in 3T3-L1 adipocytes. 
Connection between enzymatic activation and subcellular localization. The Journal of Biological Chemistry. 275: 40148-40154.

Johnson, L.H. (1992). Glycogen phosphorylase: control by phosphorylation and allosteric effectors. FASEB Journal. 6: 2274-2282.

Jones, M. and Ferrans, V.J. (1973). Intramitochondrial glycogen in hypertrophied infundibular muscle of patients with congenital heart diseases. American Journal of Pathology. 70: 69-88.

Jucker, B.M., Barucci, N. and Shulman, G.I. (1999). Metabolic control of insulinstimulated glucose disposal in rat skeletal muscle. American Journal of Physiology (Endocrinology and Metabolism). 277: E505-E512.

Karasaki, S. (1971). Cytoplasmic and nuclear glycogen synthesis in novikoff ascites hepatoma cells. Ultrastructure Research. 35: 181-196.

Kawanaka, K., Han, D.H., Nolte, L.A., Hansen, P.A., Nakatani, A. and Hollowsky, J.O. (1999). Decreased insulin-stimulated GLUT-4 translocation in glycogensupercompensated muscles of exercised rats. American Journal of Physiology (Endocrinology and Metabolism). 276: E907-E912.

Kawanaka, K., Nolte, L.A., Han, D.H., Hansen, P.A. and Hollosky, J.O. (2000). Mechanisms underlying impaired GLUT-4 translocation in glycogen-supercompensated muscles of exercised rats. American Journal of Physiology (Endocrinology and Metabolism). 277: E1103-E1110. 
Kayar, S.R., H. Hoppeler, H. Claassen, and F. Oberholzer. (1986). Acute effects of endurance exercise on mitochondrial distribution and skeletal muscle morphology. European Journal of Applied Physiology. 54: 578-584.

Keller, P.J. and Cori, G.T. (1953). Enzymatic conversion of phosphorylase a to phosphorylase b. Biochimica and Biophysica Acta. 12: 235-238.

Kennedy, L.D., Kirkman, B.R., Lomako, J., Rodriguez, I.R. and Whelan, W.J. (1985). The biogenesis or rabbit muscle glycogen. In: Membranes and Muscle, edited by Berman et al. Oxford ICSU/IRL, p. 65-84.

Klip, A., Volchuk, A. Lijing, H. and Tsakiridis, T. (1996). The glucose transporters of skeletal muscle. Seminar in Cell and Developmental Biology. 7: 229-237.

Koval, J.A., DeFronzo, R.A., O'Doherty, R.M., Printz, R., Ardehali, H., Granner, D.K. and Mandarino, L.J. (1998). Regulation of Hexokinase II activity and expression in human muscle by moderate exercise. American Journal of Physiology (Endocrinology and Metabolism). 274: E304-E308.

Kraniou, Y., Cameron-Smith, D., Misso, M., Collier, G. and Hargreaves, M. (2000). Effects of exercise on GLUT-4 and glycogenin gene expression in human skeletal muscle. Journal of Applied Physiology. 88: 794-796.

Kruszynska, Y.T., Ciaraldi, T.P. and Henry, R.R. (2001). Regulation of glucose metabolism in skeletal muscle. in : Handbook of Physiology: A critical, comprehensive presentation of physiological knowledge and concepts (Section 7: The endocrine 
system), edited by Jefferson et al., 579-607.

Kuo, C.-H., Browning, K.S. and Ivy, J.L. (1999). Regulation of GLUT 4 protein expression and glycogen storage after prolonged exercise. Acta Physiologica Scandinavica. 165: 193-201.

Landon, D.N. (1992). Skeletal muscle - normal morphology, development and innervation. In: Skeletal Muscle Pathology, edited by F.L. Mastaglia and L.W. Detchant. London: Churchill Livingstone. Chapter 1: 1-40.

Lane, R.D., Hegazy, M.G. and Reimann, E.M. (1989). Subcellular localisation of glycogen synthase with monoclonal antibodies. Biochemistry International. 18: 961 970.

Laurent, D., Hundal, R.S., Dresner, A., Price, T.B., Vogel, S.M., Petersen, K.F. and Shulman, G.I. (2000). Mechanism of muscle glycogen autoregulation in humans. American Journal of Physiology (Endocrinology and Metabolism). 278: E663-E668.

Lazarow, A. (1942). Particulate glycogen: a submicroscopic component of the guina pig liver cell; its significance in glycogen storage and the regulation of blood sugar. Anatomical Record. 84: 31-50.

Leloir, L.F. and Goldemberg, .S.H. (1960). Synthesis of glycogen from uridine diphosphate glucose in liver. Journal of Biological Chemistry. 235: 919-923. 
Lexell, J., Taylor, C.C. and Sjostrom, M. (1988). What is the cause of the aging atrophy? Total number, size and proportion of different fiber types studied in whole vastus lateralis muscle from 15- to 83-year-old men. Joumal of the Neurological Sciences. 84: 275-294.

Lomako, J., Lomako, W.M. and Whelan, W.J. (1988). A self-glucosylating protein is the primer for rabbit muscle glycogen biosynthesis. FASEB Journal. 2: 3097-3103.

Lomako, J., Lomako, W.M. and Whelan, W.J. (1990). Substrate specificity of the autocatalytic protein that primes glycogen synthesis. FEBS Letters. $264: 13-16$.

Lomako, J., Lomako, W.M. and Whelan, W.J. (1991). Proglycogen: a low-molecularweight form of muscle glycogen. FEBS Letters. 279: 223-228.

Lomako, J., Lomako, W.M., Whelan, W.J., Dombro, R.S., Neary, J.T. and Nosrenberg, M.D. (1993). Glycogen synthesis in the astrocyte: from glycogenin to proglycogen to glycogen. FASEB Journal. 7: 1386-1393.

Lomako, J. and Whelan, W.J. (1988). The occurrence of serine phosphate in glycogenin: a possible regulatory site. Biofactors. 1: 261-264.

Luft, J.H. (1956). Permanganate, a new fixative for electron microscopy. Journal of biophysical and biochemical cytology. 2: 799-802.

Lund, S. Holman, G.D., Schmitz, O. and Pederson, O. (1993). Glut 4 content in the plasma membrane of rat skeletal muscle: Comparative studies of the subcellular 
fraction-method and the exofacial photolabelling technique using ATB-BMPA. FEBS letters. 330: 312-318.

Madsen, N.B. (1986). Glycogen phosphorylase. In: The enzymes ( $3^{\text {rd }}$ ed.), edited by P. Boyer and E.G. Krebs. Orlando, FL: Academic. 17: 365-394.

Madsen, N.B. and Cori, G.T. (1956). The interaction of muscle phosphorylase with pchloromercuribenzoate. I. Inhibition of activity and effect on the molecular weight. Journal of Biological Chemistry. 223: 1055-1065.

Madsen, N.B. and Cori, G.T. (1958). The binding of glycogen and phosphorylase. Journal of Biological Chemistry. 233: 1251-1256.

Magnusson, I., Rothman, D.L., Jucker, B., Cline, G.W., Shulman, R.G. and Shulman, G.l. (1994). Liver glycogen turnover in fed and fasted man. American Journal of Physiology (Endocrinology and Metabolism). 266: E796-E803.

Marchand, I., Chorneyko, K., Tarnopolsky, M., Hamilton, S., Shearer, J., Potvin, J. and Graham, T.E. (2001). Quantification of subcellular glycogen in resting human muscle: granule size, number and location. Submitted to Journal of Applied Physiology.

Maron, B.J. and Ferrans, V.J. (1974). The occurrence of $\alpha$-glycogen in human cardiac muscle cells. Journal of Molecular and Cellular Cardiology. 6: 85-89.

Martinuzzi, A., Schievano, G., Nascimbeni, A. and Fanin, M. (1999). McArdle's disease: the unsolved mystery of the reappearing enzyme. American Journal of Pathology. 154: 
$1893-1897$.

Maunsbach, A.B. and Afzeliuz, B.A. (1999). Biomedical Electron Microscopy: Illustrated Methods and Interpretations. Academic Press.

McCartney, N., Spriet, L.L., Heigenhauser, G.J.F., Kowalchuk, J.M., Sutton, J.R. and Jones, N.L. (1986). Muscle power and metabolism in maximal intermittent exercise. Journal of Applied Physiology. 60: 1164-1169.

Melendez, R., Melendez-Hevia, E. \& Canela, E.I. (1999). The fractal structure of glycogen: a clever solution to optimize cell metabolism. Biophysical Journal. 77: 13271332.

Melendez, R., Melendez-Hevia, E. \& Cascante, M. (1997). How did glycogen structure evolve to satisfy the requirement for rapid mobilization of glucose? A problem of physical constraints in structure building. Journal of Molecular Evolution. 45: 446-455.

Melendez-Hevia, E., Waddell, T.G. \& Shelton, E.D. (1993). Optimization of molecular design in evolution of metabolism: the glycogen molecule. Biochemistry Journal. 295: 477-483.

Meyer, F., Heilmeyer, L.M.G., Haschke, R.H. \& Fisher, E.H. (1970). Control of phosphorylase activity in a muscle glycogen particle: Isolation and characterization of the protein-glycogen complex. The Journal of Biological Chemistry. 245: 6642-6648. 
Montell, E., Arias, A. and Gomez-Foix, A.M. (1999). Glycogen depletion rather than glucose 6-P increments controls early glycogen recovery in human cultured muscle. American Joumal of Physiology (Regulatory Integrative and Comparative Physiology). 276: R1489-R1495.

Mordoh, J., Leloir, L.F. and Krisman, C.R. (1965). In vitro synthesis of particulate glycogen. Proceedings of national Academy of Sciences. 53: 86-91.

Morgan, H.E. and Parmeggiani, A. (1964). Regulation of glycogenolysis in muscle. In: Control of Glycogen Metabolism, edited by Whelan, W.J. London: Churchill Livingstone, p. 254-272.

Munger, R., Temler, E., Jallut, D., Haesler, E. and Felber, J.P. (1993). Correlations of glycogen synthase and phosphorylase activities with glycogen concentration in human muscle biopsies. Evidence for a double-feedback mechanism regulating glycogen synthesis and breakdown. Metabolism. 42: 36-43.

Nelson, T.El, White, R.C. and Watts, T.E. (1972). The action of the glycogen debranching enzyme system in a muscle protein particle. Biochemical and Biophysical research communications. 47 : 254-259.

Newgard, C.B., Brady, M.J., O'Doherty, R.M. and Saltiel, A.R. (2000). Organizing glucose disposal. Emerging roles of the glycogen targeting subunits of protein phosphatase-1. Diabetes. 49: 1967-1977.

Newsholme, E.A. and Leech, A.R. (1983). Biochemistry for the medical sciences. 
Toronto, Wiley.

Nielsen, J.N., Derave, W., Kristiansen, S., Ralston, E., Ploug, T. and Richter, E.A. (2001). Glycogen synthase localization and activity in rat skeletal muscle is strongly dependent on glycogen content. Journal of Physiology. 531: 757-769.

Nilsson, L.H. and E. Hultman. (1973). Liver glycogen in man - the effect of total starvation or a carbohydrate-poor diet followed by carbohydrate feeding. Scandinavian journal of clinical and laboratory investigation. 32: 325-330.

O'Doherty, R.M., Jensen, P.B., Anderson, P., Jones, J.G., Berman, H.K., Kearney, D., and Newgard, C.B. (2000). Activation of direct and indirect pathways of glycogen synthesis by hepatic overexpression of protein targeting to glycogen. Journal of Clinical Investigations. 105: 479-488.

Payne, C.M., L.Z. Stern, R.G. Curless, and L.K. Hannapel. (1975). Ultrastructural fibre typing in normal and diseased human muscle. Journal of Neurological Science. 25: 99108.

Pette, D. and Staron, R.S. (1990). Cellular and molecular diversities of mammalian skeletal muscle fibers. Reviews of Physiology Biochemistry and Pharmacology. 116: 176.

Pickett-Gies, C.A. and Walsh, D.A. (1986). Phosphorylase kinase. In: The Enzymes ( $3^{\text {rd }}$ ed.), edited by Boyer and Krebs. Orlando, PF: Academic, vol. 17, p. 395-459. 
Piehl, K. (1974). Time course of refilling of glycogen stores in human muscle fibres following exercise-induced glycogen depletion. Acta Physiologica Scandinavica. 90: 297-302.

Piras, R., Rothman, L.B. and Cabib, E. (1968). Regulation of muscle glycogen synthetase by metabolites. Differential effects of the I and D forms. Biochemistry. 7 : 56-66.

Pitcher, J., Smythe, C., Campbell, D.G. and Cohen, P. (1987). Identification of the 38kDa subunit of rabbit skeletal muscle glycogen synthase as glycogenin. European Journal of Biochemistry. 169: 497-502.

Pitcher, J., Smythe, C. and Cohen, P. (1988). Glycogenin is the priming glucosyltransferase required for the initiation of glycogen biogenesis in rabbit skeletal muscle. European Journal of Biochemistry. 176: 391-395.

Plaxton, W.C. and Storey, K.B. (1986). Glycolytic enzyme binding and metabolic control in anaerobiosis. Journal of Comparative Physiology (Biochemical, Systemic, and Environmental Physiology). 156: 635-640.

Polishchuk, S.V., Brandt, N.R., Meyer, H.E., Varsanyi, M. and Heilmeyer, L.M.G. Jr. (1995). Does phosphorylase kinase control glycogen biosynthesis in skeletal muscle. FEBS letters. 362: 271-275.

Price, T.B., Laurent, D., Petersen, K.F., Rothman, D.L. and Shulman, G.I. (2000). Glycogen loading alters muscle glycogen resynthesis after exercise. Journal of Applied 
Physiology. 88: 698-704.

Price, T.B., Rothman, D.L., Avison, M.J., Buonamico, P. and Shulman, R.G. (1991). 13C- NMR measurements of muscle glycogen during low-intensity exercise. Journal of Applied Physiology. 70: 1836-1844.

Price, T.B., Taylor, R., Mason, G.F., Rothman, D.L., Shulman, G.I. and Shulman, R.G. (1994). Turnover of human glycogen with low-intensity exercise. Medicine and Science in Sports and Exercise. 26: 983-991.

Raz, I. Katz, A. and Dpencer, M.K. (1991). Epinephrine inhibits insulin-mediated glycogenesis but enhances glycolysis in human skeletal muscle. American Journal of Physiology (Endocrinology and Metabolism). 260: E430-E435.

Ren, J.M. and Hultman, E. (1989). Regulation of glycogenolysis in human skeletal muscle. Journal of Applied Physiology. 67: 2243-2248.

Ren, J.M. and Hultman, E. (1990). Regulation of phosphorylase a activity in human skeletal muscle. Journal of Applied Physiology. 69: 919-923.

Revel, J.-P., Napolitano, L. and Fawcett, W. (1960). Identification of glycogen in electron micrographs of thin tissue sections. The Journal of Biophysical and Biochemical Cytology. 8: 575-589.

Revel, J.P. (1964). Electron Microscopy of glycogen. The Journal of Histochemistry and Cytochemistry. 12: 104-114. 
Richter, E.A. (1996). Glucose utilization. In : Handbook of Physiology: Exercise: Regulation and integration of multiple systems (Section 12), edited by Rowell and Shepherd, p. 912-951.

Richter, E.A., Ploug, T. and Galbo, H. (1985). Increased muscle glucose uptake after exercise. No need for insulin during exercise. Diabetes. 34: 1041-1048.

Roach, P.J. (1990). Control of glycogen synthase by hierarchal protein phosphorylation. FASEB Journal. 4: 2961-2968.

Roach, P.J., Cheng, C., Huang, D., Lin, A., Mu, J., Skurat, A.V., Wilson, W. and Zhai, L. (1998). Novel aspects of the regulation of glycogen storage. Journal of Basic and Clinical Physiology and Pharmacology. 9: 139-151.

Roach, P.J. and Skurat, A.V. (1997). Self-glucosylating initiator proteins and their role in glycogen biosynthesis. Progress in Nucleic Acid Research and Molecular Biology. 57: 289-317.

Roach, P.J., Skurat, A.V. and Harris, R.A. (2001). Regulation of glucose metabolism in skeletal muscle. In : Handbook of Physiology: A critical, comprehensive presentation of physiological knowledge and concepts (Section 7: The endocrine system), edited by Jefferson et al., p.609-647.

Robinson, J.M., Karnovsky, N.L. and Karnovsky, M.J. (1982). Glycogen accumulation in polymorphonuclear leukocytes, and other intracellular alterations that occur during inflammation. The Journal of Cell Biology. 95: 933-942. 
Rybicka, K.K. (1979). Glycosomes (protein-glycogen complex) in the canine heart. Ultrastructure, histochemistry and changes induced by acidic treatment. Virchows archiv B-cell pathology. 30: 335-447.

Rybicka, K.K. (1981a). Simultaneous demonstration of glycogen and protein in glycosomes of cardiac tissues. The Journal of Histochemistry and Cytochemistry. 29: 4-8.

Rybicka, K.K. (1981b). Binding of glycosomes to Endoplasmic reticulum and to intermediate filaments in cardiac conduction fibers. The Journal of Histochemistry and Cytochemistry. 29: 553-560.

Rybicka, K.K. (1996). Glycosomes - the organelles of glycogen metabolism. Tissue and Cell. 28: 253-265.

Schmalbruch, H. \& Kamieniecka, Z. (1974). Fibre types in the human brachial biceps muscle. Experimental Neurology. 44: 313-328.

Schulz, A.R. (1998). Control analysis of muscle glycogen metabolism. Archives of Biochemistry and Biophysics. 353: 172-180.

Scott, R.8. \& Still, W.J.S. (1968). Glycogen in human peripheral blood leucocytes. II. The macromolecular state of leucocyte glycogen. Journal of Clinical Investigations. 47: 353-359.

Shearer, J. Marchand, I. Sathasivam, P. Tarnopolsky, M.A. and Graham, T. (2000). 
Glycogenin activity in human skeletal muscle is proportional to muscle glycogen concentration. American Journal of Physiology (Endocrinology and Metabolism). 278: E177-E180.

Shearer, J., Marchand, I., Tarnopolsky, M.A., Dyck, D.J. and Graham, T. (2001). Proand macro-glycogenolysis during repeated exercise: roles of glycogen content and phosphorylase activation. Journal of Applied Physiology. 90: 880-888.

Shulman, G.I., Rothman, D.L., Jue, T., Stein, P. DeFronzo, R.A. and Shulman, R.G. (1990). Quantitation of muscle glycogen synthesis in normal subjects and subjects with Non-Insulin-Dependent Diabetes by $13 \mathrm{C}$ nuclear magnetic resonance spectroscopy. New England Journal of Medicine. 322: 223-228.

Simoneau, J., S.R. Colberg, F.L. Thaete, and D.E. Kelley. (1995). Skeletal muscle glycolytic and oxidative enzyme capacities are determinants of insulin sensitivity and muscle composition in obese women. FASEB Journal. 9: 273-278.

Sjostrom, M., Friden, J. and Ekblom, B. (1982a). Fine structural details of human muscie fibres after fibre type specific glycogen depletion. Histochemistry. 76: 425-438.

Sjostrom M., S. Kidman, K. Henriksson Larsen, and K.-A. Angquist. (1982). Z- and Mband appearance in different histochemically defined types of human skeletal muscle fibers. The Journal of Histochemistry and Cytochemistry. 30: 1-11.

Skurat, A.V., Lim, S.-S. and Roach, P.J. (1997). Glycogen biogenesis in rat 1 fibroblasts expressing rabbit muscle glycogenin. European Joumal of Biochemistry. 
245: $147-155$.

Smythe, C., Caudwell, F.B., Ferguson, M. and Cohen, P. (1988). Isolation and structural analysis of a peptide containing the novel tyrosyl-glucose linkage in glycogenin. EMBO Journal. 7: 2681-2686.

Smythe, C., Villar-Palasi, C. and Cohen, P. (1989). Structural and functional studies on rabbit liver glycogenin. European Joumal of Biochemistry. 183: 205-209.

Smythe, C. and Cohen, P. (1991). The discovery of glycogenin and the priming mechanism for glycogen biogenesis. European Journal of Biochemistry. 200: 625-631.

Smythe, C., Watt, P. and Cohen, P. (1990). Further studies on the role of glycogen biosynthesis. European Journal of Biochemistry. 189: 199-204.

Soderlund, K., Greenhaff, P.L. and Hultman, E. (1992). Energy metabolism in type I and type II human muscle fibers during short term electrical stimulation at different frequencies. Acta Physiologica Scandinavica. 144: 15-22.

Steel, R.G.D. and J.H. Torrie. (1960). Principles and procedures of statistics: with special reference to the biological sciences. New York: McGraw-Hill Book Co.

Suzuki, Y., Lanner, C., Kim, J.-H., Vilardo, P.G., Zhang, H., Yang, J., Cooper, L.D., Steele, M., Kennedy, A., Bock, C.B., Scrimgeour, A., Lawrence, J.C. and DePaoliRoach, A.A. (2001). Insulin control of glycogen metabolism in knockout mice lacking the muscle-specific protein phosphatase PP1G/R $R_{\mathrm{GL}}$. Molecular and Cellular Biology. 21: 
2683-2694.

Tang, P.M., Bondor, J.A., Swiderek, K.M. and DePaoli-Roach, A.A. (1991). Molecular cloning and expression of the regulatory $\left(R_{\mathrm{GL}}\right)$ subunit of the glycogen-associated protein phosphatase. The Journal of Biological Chemistry. 266: 15782-15789.

Taylor, C., Cox, A.J., Kernohan, J.C. and Cohen, P. (1975). Debranching enzyme from rabbit skeletai muscle. Purification, properties and physiological role. European Journal of Biochemistry. 51: 105-115.

Thiery, J.-P. (1967). Mise en évidence des polysaccharides sur coupes fines en microscopie électronique. Journal de Microscopie. 6: 987-1018.

Tsintzas, O.-K., C. Williams, L. Boobis, and P. Greenhaff. (1996). Carbohydrate ingestion and single muscle fibre glycogen metabolism during prolonged running in men. Journal of Applied Physiology. 81: 801-809.

Vandenberghe, K. Richter, E.A. and Hespel, P. (1999). Regulation of glycogen breakdown level in contracting rat muscle. Acta Physiologica Scandinavica. 165: 307 . 314.

Villar-Palasi, C. (1969). Oligo- and polysaccharide inhibition of muscle transferase D phosphatase. Annals of the New York Academy of Sciences. 116: 719-730.

Vollestad, N.K. and Blom, P.C.S. (1985). Effect of varying exercise intensity on glycogen depletion in human muscle fibres. Acta Physiologica Scandinavica. 125: 395- 
405.

Vollestad, N.K., Blom, P.C.S. and Gronnerod, O. (1989). Resynthesis of glycogen in different muscle fibre types after prolonged exhaustive exercise in man. Acta Physiologica Scandinavica. 137: 15-21.

Vollestad, N.K., Tabata, I. and Medbo, J.I. (1992). Glycogen breakdown in different human muscle fire types during exhaustive exercise of short duration. Acta Physiologica Scandinavica. 144: 135-141.

Vollestad, N.K., O. Vaage, and L. Hermansen. (1984). Muscle glycogen depletion patterns in type I and subgroups of type $\mid 1$ fibres during prolonged severe exercise in man. American Journal of Physiology (Endocrinology and Metabolism). 122: 433-44.

Walsh, T.P., Masters, C.J., Morton, D.J. and Clarke, F.M. (1981). The reversible binding of glycolytic enzymes in ovine skeletal muscle in response to tetanic stimulation. Biochimica et Biophysica Acta. 675: 29-39.

Wanson, J.-C. \& Drochmans, P. (1968). Rabbit skeletal muscle glycogen: a morphological and biochemical study of glycogen $\beta$-particles isolated by the precipitation-centrifugation method. The Journal of Cell Biology. 38: 130-150.

Wanson, J.-C. \& Drochmans, P. (1972). Role of the sarcoplasmic reticulum in glycogen metabolism. Binding of phosphorylase, phosphorylase kinase, and primer complexes to the sarcovesicles of rabbit skeletal muscle. The Journal of Cell Biology. 54, 206-224. 
White, M.G. and D.H. Snow. (1987). Quantitative histochemical study of glycogen depletion in the maximally exercised Thoroughbred. Equine Veterinary Joumal. 19: 6769.

Williams, M.A. (1977). Quantitative methods in biology. In: Practical methods in electron microscopy (vol. 6, Chapter 2), Anonymous Cambridge: North-Holland Publishing Company, p 68.

Wilson, J.E. (1994). Hexokinase. Review of Physiology Biochemistry and Pharmacology. 126: 65-174.

Xu, K.Y. and Becker, L.C. (1998). The ultrastructural localization of glycolytic enzymes on sarcoplasmic reticulum vesicles. The Journal of Histochemistry and Cytochemistry. 46: $419-427$

Young, J.C., Garthwaite, S.M., Bryan, J.E., Cartier, L.J. and Holloszy, J.O. (1983). Carbohydrate feeding speeds reversal of enhanced glucose uptake in muscle after exercise. American Journal of Physiology (Regulatory Integrative and Comparative Physiology). 245: R684-R688.

Zierler, K. (1999). Whole body glucose metabolism. American Joumal of Physiology (Endocrinology and Metabolism). 276: E409-E426. 
Appendix A 


\begin{tabular}{|c|c|c|c|c|}
\hline \multicolumn{5}{|c|}{ Total Glycogen Area (um x um) } \\
\hline Image & Value & Cumulative mean & Cumulative S.D. & Cumulative C.V. \\
\hline $\begin{array}{l}d 1 c 1-a \\
d 1 c 1-b \\
d 1 c 2-a \\
d 1 c 2-b \\
d 1 c 3-a \\
d 1 c 3-b \\
d 1 c 4-a \\
d 1 c 4-b \\
d 1 c 5-a \\
d 1 c 5-b \\
d 1 c 6-a \\
d 1 c 6-b \\
d 1 c 7-a \\
d 1 c 7-b \\
d 1 c 8-a \\
d 1 c 8-b\end{array}$ & $\begin{array}{l}0.480 \\
0.427 \\
0.803 \\
0.498 \\
0.590 \\
0.493 \\
0.652 \\
0.617 \\
0.519 \\
0.521 \\
0.301 \\
0.382 \\
0.639 \\
0.450 \\
0.645 \\
0.523\end{array}$ & $\begin{array}{l}0.453 \\
0.570 \\
0.552 \\
0.560 \\
0.549 \\
0.563 \\
0.570 \\
0.564 \\
0.560 \\
0.532 \\
0.527 \\
0.535 \\
0.534 \\
0.537 \\
0.534\end{array}$ & $\begin{array}{l}0.137 \\
0.104 \\
0.170 \\
0.148 \\
0.135 \\
0.130 \\
0.121 \\
0.115 \\
0.109 \\
0.130 \\
0.127 \\
0.126 \\
0.122 \\
0.130 \\
0.122\end{array}$ & $\begin{array}{c}8.3 \\
35.7 \\
30.8 \\
26.5 \\
24.7 \\
23.0 \\
21.3 \\
20.4 \\
19.5 \\
24.4 \\
24.0 \\
23.5 \\
22.8 \\
24.2 \\
22.8\end{array}$ \\
\hline \multicolumn{5}{|c|}{ Intra-myofibrillar Glycogen Area (um x um) } \\
\hline Image & Value & Cumulative mean & Cumulative S.D. & Cumulative C.V. \\
\hline d1c1-a & 0.023 & & & \\
\hline$d t c t-b$ & 0.070 & 0.046 & 0.034 & 72.7 \\
\hline dic2-a & 0.074 & 0.056 & 0.029 & 51.7 \\
\hline$d 1 c 2-b$ & 0.056 & 0.056 & 0.024 & 42.2 \\
\hline d1c3-a & 0.085 & 0.071 & 0.024 & 33.7 \\
\hline d1c3-b & 0.082 & 0.067 & 0.024 & 36.3 \\
\hline dic4-a & 0.103 & 0.064 & 0.025 & 40.0 \\
\hline d1c4-b & 0.071 & 0.062 & 0.025 & 41.2 \\
\hline dic5-a & 0.041 & 0.062 & 0.024 & 39.3 \\
\hline d1c5-b & 0.033 & 0.062 & 0.025 & 41.2 \\
\hline d1c6-a & 0.039 & 0.058 & 0.023 & 39.5 \\
\hline d1c6-b & 0.052 & 0.059 & 0.023 & 37.9 \\
\hline dtc7-a & 0.042 & 0.059 & 0.023 & 37.9 \\
\hline$d t c 7-b$ & 0.048 & 0.059 & 0.022 & 36.8 \\
\hline dic8-a & 0.072 & 0.061 & 0.024 & 40.1 \\
\hline d1c8-b & 0.056 & 0.059 & 0.022 & 36.8 \\
\hline
\end{tabular}

Example of calculation to determine the minimal number of images required to represent whole fibre myofibrillar glycogen area. The first column is the code for each image involved in the analysis. After six negatives (12 images (i.e. $a$ and b)) per fibre, for both the "Total Glycogen Area" and "Intramyofibrillar Glycogen Area", the addition of extra images did not further decrease the coefficient of variation. Six negatives ( 12 images) per fibre were therefore chosen as the minimal number necessary to represent the muscle fibre as a whole. 
Appendix $B$ 
The software used for digitising purposes was "Labview ${ }^{\circledR}$ ". A description of the relevant settings used include:

- In "Set up": $\quad$-Current driver = UVP capture 128

-NTSC

-Composite

- Camera = cooled monochrome

-There is something else that says 250 (verify)

- In "Signal": $\quad$-Brightness $=34$

-Contrast $=35$

-Hue $=0$

-Saturation $=32$

- In "integration": -Check dynamic integration

- Check Autl exposure

- Check Saturation warning

-DO NOT check Integrate

- In "Preview": -DO NOT check Saturation preview

- In "Image": $\quad$-Check New Image 
Appendix C 
The following Macros (from Image Pro Plus (8) in IPBasic language) were created by $\mathrm{Dr}$ Jim Potvin and applied to every image during the appropriate step of the computer analysis procedures (see section "Detailed computer analysis procedures").

\section{Preparing the image}

Sub Ingrid_process()

$$
\begin{aligned}
& \text { ret }=\text { IpDocMaximize () } \\
& \text { ret }=1 \mathrm{pWsScale}(537,408,0) \\
& \text { ret }=\text { IpLutShow }(1) \\
& \text { ret }=\text { IpLutSetAttr(LUT_CONTRAST },-1) \\
& \text { ret }=\mid \text { pLutApply }() \\
& \text { ret }=\text { IpLutShow }(0) \\
& \text { End Sub } \\
& \text { ret }=\text { lpHstEqualize(EQ_BESTFIT) }
\end{aligned}
$$

\section{Single particle analysis}

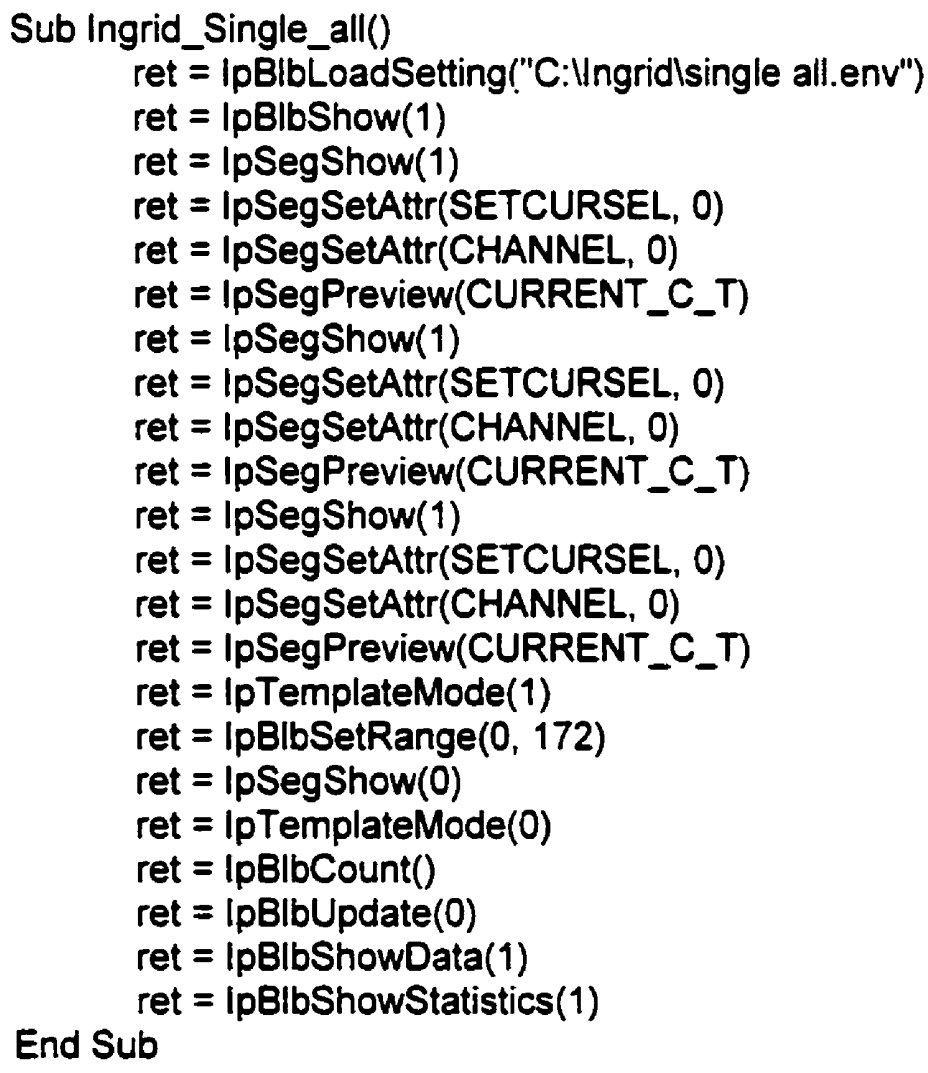




\section{Glycogen content analysis}

Sub Ingrid_total_all()

ret $=\mid$ pDocMaximize ()

ret $=\mid$ IpBIbShow $(1)$

ret = IpB|bLoadSetting("C:Vngridltotal all.env")

ret $=$ IpSegShow $(1)$

ret $=\mid$ pSegSetAttr $($ SETCURSEL, 0)

ret $=\mid$ pSegSetAttr(CHANNEL, 0$)$

ret $=$ lpSegPreview $($ CURRENT_C_T)

ret $=$ lpSegShow $(1)$

ret $=$ IpSegSetAttr(SETCURSEL, 0)

ret $=$ IpSegSetAttr $($ CHANNEL, 0$)$

ret $=$ lpSegPreview(CURRENT_C_T)

ret $=\mid \mathrm{pSegShow}(1)$

ret $=$ IpSegSetAttr(SETCURSEL, 0)

$r e t=\mid p S e g S e t A t t r(C H A N N E L, 0)$

ret $=$ IpSegPreview(CURRENT_C_T)

ret $=$ IpTemplateMode $(1)$

ret $=\mid p$ SegSetRange $(0,0,112)$

ret $=$ lpSegPreview(CURRENT_C_T)

ret $=\mid p B i b S e t R a n g e(0,112)$

ret $=\mid p$ SegShow $(0)$

ret $=\mid p$ TemplateMode $(0)$

ret $=\mid$ pBlbCount()

End Sub

ret $=|p B| b U p d a t e(0)$ 


\section{Background analysis}

Sub Ingrid_Background()

ret $=$ IpDocMaximize ()

ret $=$ IpAoiShow(FRAME_NONE)

ret $=$ IpAoiShow(FRAME_IRREGULAR)

ret $=$ IpTemplateMode $(1)$

ret $=$ lpListPts(Pts(0), "387 167386168384168382169380171378173376

175375176375177374179 ")

ret = lpMorePts("374 183374184374186375186377187378187380185

$382184384184385183 "$ ")

ret $=$ IpMorePts("386 183387182387181388181390179390177392175

392174393173393172 ")

ret $=$ lpMorePts("394 171394170393169388169386169384169 ")

ret $=\mid$ pAoiCreatelrregular $(\operatorname{Pts}(0), 36)$

ret $=\mid \mathrm{pTemplateMode}(0)$

ret $=$ IpSegShow $(1)$

ret $=$ lpSegSetAtt $($ SETCURSEL, 0)

ret $=1$ pSegSetAttr(CHANNEL, 0$)$

ret $=\mid$ pSegPreview $($ CURRENT_C_T)

ret $=\mid$ pSegShow $(1)$

ret $=1$ pSegSetAttr $($ SETCURSEL, 0$)$

ret $=\mid$ pSegSetAttr $(C H A N N E L, 0)$

ret $=\mid$ pSegPreview $($ CURRENT_C_T)

ret $=\mid$ pSegShow $(1)$

ret $=\mid p$ SegSetAttr $($ SETCURSEL, 0$)$

ret $=1$ pSegSetAttr $(C H A N N E L, 0)$

ret $=$ lpSegPreview $($ CURRENT_C_T)

ret $=1 p$ SegSetRange $(0,0,255)$

ret $=1$ pSegPreview $\left(\right.$ CURRENT $\left.C_{-} T\right)$

ret $=|\mathrm{pB}| \mathrm{bSetRange}(0,255)$

ret $=\mid$ pSegShow $(0)$

ret $=|p B| b L o a d S e t t i n g(" C:$ :Ingridltest background.env")

ret $=|p B| b$ Count ()

ret $=\mid$ pB|bUpdate $(0)$

End Sub

ret $=|p B| b S h o w S t a t i s t i c s(1)$ 
Appendix D 
Following is an example of the procedure used in the generation of missing data. The present example concerns the variable "glycogen area". In that particular example, at time $z$, subjects 3 and 5 are missing a type I fibre and subjects 2 and 4 are missing a type IIB fibre. Following is the matrix used in the generation of the missing data for that particular example.

\begin{tabular}{l|lllll} 
Time z & Subject 1 & Subject 2 & Subject 3 & Subject 4 & Subject 5 \\
\hline Fibre type I & 0.065 & 0.061 & & 0.050 & \\
Fibre type IIA & 0.076 & 0.074 & 0.070 & 0.080 & 0.069 \\
Fibre type IIB & 0.070 & & 0.065 & & 0.074
\end{tabular}

As an example, let us examine the situation where type IIB of subject 2 is missing (at time z):

- The average type IIB glycogen area value from all subjects whose type IIB was present at time $\mathbf{z}$ was first calculated:

$\rightarrow$ Average type IIB (from subjects 1,3 , and 5$)=(0.070+0.065+0.074) / 3=0.070$

- Then, the relationship between subjects was taken into account by calculating the ratio of the missing subject's type IIA value over the average of all type llA values of the subjects that did possess the missing fibre type IIB:

$\rightarrow$ Average type IIA (from subjects 1,3 , and 5$)=(0.076+0.070+0.069) / 3=0.072$

$\rightarrow$ Relationship between subjects as a ratio $=$

Type IIA (subjects 2)/ Average type \|A (from subjects 1, 3, and 5) =

$$
0.74 /[(0.076+0.070+0.069) / 3]=0.074 / 0.072=1.028
$$


$\rightarrow$ It is worth mentioning that the type IIA was used as a reference since it was the only one of the three fibre types that was present in every selected samples.

- The missing IIB giycogen area value for subject 2 at time $z$ was then estimated by multiplying this ratio (representing the relationship between subjects) by the average type IIB value from all type IIB present (calculated above):

$\rightarrow 1.028 \times 0.070=0.072$

- The procedure was repeated for every missing value.

- This technique was use to generate $75 \%$ of type IIB and $10 \%$ of type I fibres. No type IIA value had to be generated. 
Appendix E 
Following are detailed instructions for SAS and explanation of the statistical output produced by a SAS analysis.

REML Estimation Iteration History.

The convergence criteria must be met.

Covariance Parameter Estimates (REML):

- $\quad$ Subject $=$ variance between subjects.

- $\quad$ Subject $\times$ Time $=$ error term (required in all models where Time is fixed effect; that is all but the $1^{\text {st }}$ study).

$\rightarrow$ In general we want these variance components to be as small as possible.

$\rightarrow$ Both Subject and Subject $x$ time go in the random statement.

Model Fitting Information for variable $X$ :

- In general, tells you what is the best model for my data.

$\rightarrow$ Akaike's Information Criterion $(\mathrm{AIC})=$ we want it to be as close as possible to

1. It compares models.

$\rightarrow-2$ Res Log Likelihood = not important for our analysis.

\section{Solution for Fixed Effects:}

- Intercepts = not important for our analysis.

Tests of Fixed Effects:

- NDF = numerator degrees of freedom.

- $\quad$ DDF = denominator degrees of freedom. 
Least Squares Means:

- $\operatorname{Pr}>|t|=T$-test to compare whether the individual mean is different from 0 (not important for our analysis).

Contrast statements:

- It uses F-test to compare what ever we want it to compare. So in order to get specific pair-wise comparison, we are better to include a specific statement (pdiff adjust $=$ tukey) in the "Least Square Means" statement which allows me to choose the post hoc that I want (Tukey in this example).

Other details:

- The first statement is simply to identify the sheet of data

For example:

data Sheet500;

- input $=$ statement to name all the column variables, whether they are included or not in the fixed and random statements.

$\rightarrow \mathbf{S}=$ Must put in front of the discrete variables (those not numerical continuous) in the first statement before the data.

For example:

input Subject\$ Fibre\$ Time Zline Fibetype\$ Subloc\$ Myoreg\$ Glycarea Numbpart;

- $\quad$ cards; = must write just before the data.

- $\quad:=$ necessary at the end of the data.

- $\quad$ proc mixed data $=$ name of the sheet as named in the first statement. 
For example:

proc mixed data=Sheet500;

- $\quad$ class = statement to simply enumerate all the effects in which we am interested.

$\rightarrow$ In this statement, we must include all the effects (fixed and random) which will appear in the model and random statements to come.

For example:

class Subject Time Fibetype Subloc Myoreg;

- $\quad$ model $=$ statement to present the fixed effects and their interactions.

$\rightarrow$ Must add "/solution" at the end after the semicolon in order to get the solution.

$\rightarrow$ The first word after model is the dependent variable of interest.

For example:

model Numbpart= Time Fibetype Subloc Myoreg Time"Myoreg Fibetype"Myoreg Subloc*Myoreg;

$\rightarrow$ Very important for the model statement: In order to know which one of the fixed effects and which one of their interactions should be included, we have to try the different combinations starting with putting them all in (all fixed effects and interactions). Then we should look at the "Akaike's Information Criterion" in the "Model fitting Information" and aim at a value as close as possible from 1. We should then one by one remove the interactions and fixed effects that we are not interested in and look at the change in the "Akaike's Information Criterion". We should then select the best model for our data based on the lowest "Akaike's Information Criterion" value. 
- random $=$ statement for the covariate parameters or all the effects which were randomly determined (must include both Subject and Subject $x$ Time).

For example:

random subject subject"time;

- Ismeans = provides with the averages and S.D. for all the groups of data compared

$\rightarrow$ We must put "/pdiff adjust=tukey" at the end of the statement in order to get the post-hoc results.

$\rightarrow$ We can choose the post hoc that we want by changing Tukey for which ever post hoc we prefer.

For example:

Ismeans Subloc Myoreg Subloc"Myoreg /pdiff adjust=tukey;

- To end my program, we must add a "run" statement at the end.

For example:

run;

- To clear the text (since it accumulates in the same file if we do not), we must go in Global menu and select either Program editor, Log, or Output; then we must go to Edit and clear all text.

- Proc $\mathrm{glm}=$ would stand for a General Linear Model.

- $\quad$ Response variable $=$ data or dependent variable

$-\quad-=$ put in front of a line to ignore it.

- If we have a missing datum, we must put a dot and not 0 . 
Appendix F 
Following is a summary of the data sheet used for statistical analysis, as well as the parameters, variables, and effects of interest for each:

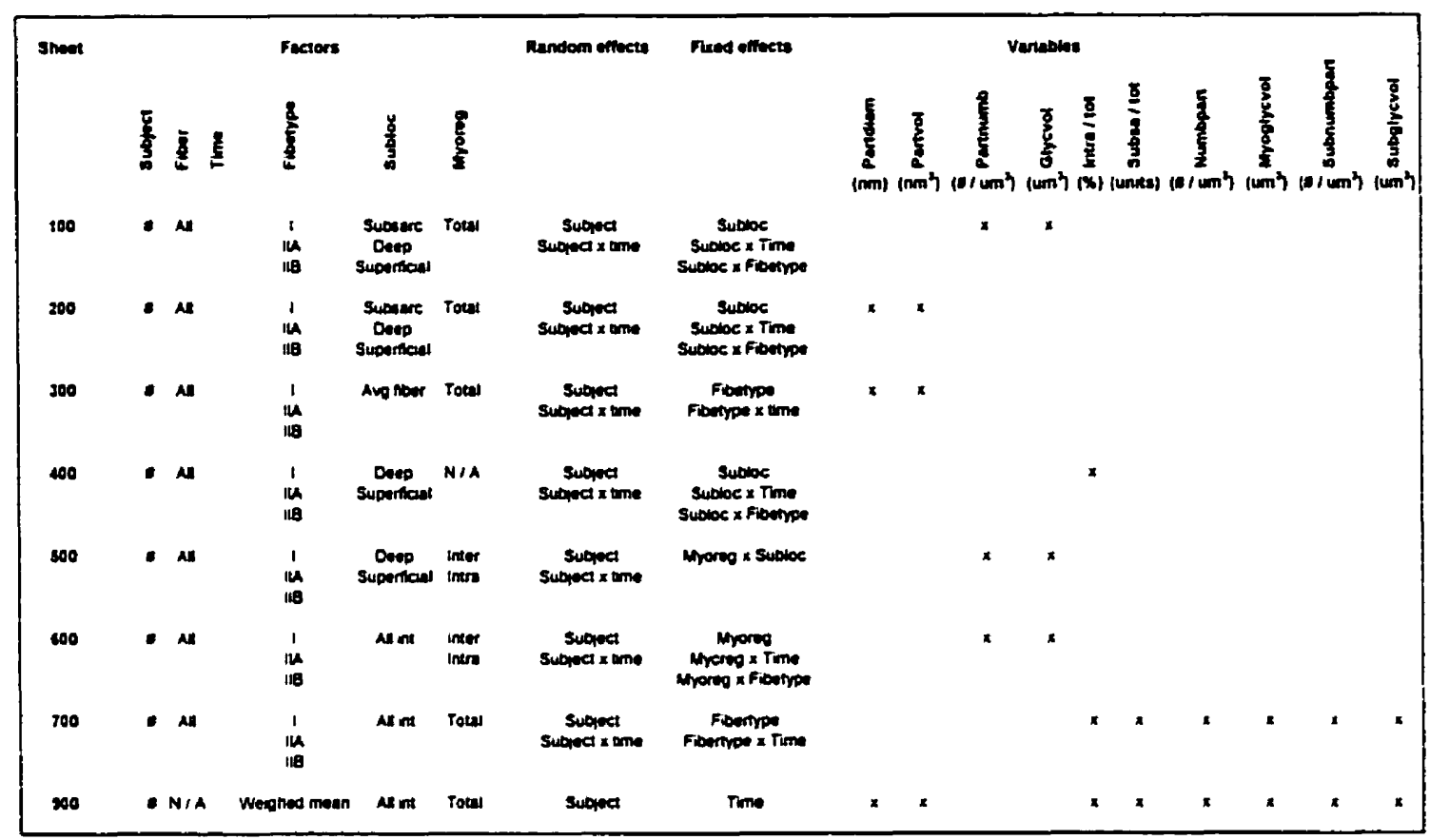

Sheet is the name of the statistical analysis sheet as used in SAS. All "Factors", "Random effects", and "Fixed effects" are displayed as described in the statistical section. The "Variables" represent all the dependent variables as described in the "Dependent variables" section. Subloc = subcellular location. Myoreg $=$ myofibrillar region. Partdiam $=$ single particle diameter. Partvol $=$ single particle volume. Partnumb $=$ \# of glycogen particles. Glycvol $=$ glycogen volume. Intra / tot $=$ Intra $/$ Myofib. Subsarc $/$ tot $=$ Subsarc $/$ Tmyofib. Numpart $=$ myofibrillar \# of glycogen particles. Myoglycvol $=$ total myofibrillar glycogen volume. Subnumbpart = subsarcolemmal \# of glycogen particles. Subglycvol = subsarcolemmal glycogen volume. 